\title{
PROCESAMIENTO DE SEÑALES E IMÁGENES \\ BIOMÉDICAS PARA EL ESTUDIO DE LA ACTIVIDAD CEREBRAL
}

Tesis de Doctorado

\section{LEANDRO BELTRACHINI}

\author{
Presentada ante la Facultad de Ingeniería de la \\ Universidad Nacional de La Plata \\ como requisito para la obtención del grado académico de
}

\section{DOCTOR EN INGENIERÍA}

Dirección de tesis:

Director: Dr. Carlos H. Muravchik

Codirector: Dr. Nicolás von Ellenrieder

Jurado de tesis:

Dr. Hugo Leonardo Rufiner

Dr. Rodolfo Rodríguez

Fecha de defensa oral y pública: 26/03/2012 



\section{Resumen}

El estudio de la estructura y funcionamiento del cerebro humano de forma no invasiva es de sumo interés en neurociencias. Técnicas como la electro/magnetoencefalografía y la resonancia magnética por tensor de difusión surgen con tal finalidad. La electro/magnetoencefalografía es una técnica que permite estudiar la actividad electromagnética que tiene lugar en el cerebro a partir de mediciones de potencial eléctrico y campo magnético originados por la activación sincrónica de conjuntos de neuronas. Por otro lugar, la resonancia magnética por tensor de difusión es una técnica que permite realizar un análisis estructural del cerebro a partir de la medición de la movilidad de los protones de hidrógeno frente a un gradiente de campo magnético aplicado.

La predicción del potencial eléctrico o campo magnético generado por una fuente conocida lleva el nombre de problema directo de la electro/magnetoencefalografía. El problema inverso consiste en la estimación de parámetros de la fuente de actividad electromagnética del cerebro a partir de un conjunto de mediciones, inevitablemente contaminadas por ruido, del potencial eléctrico sobre el cuero cabelludo y del campo magnético en el exterior de la cabeza.

En esta tesis se estudian distintos aspectos que influyen en la calidad de la solución de los problemas directo e inverso de la electro/magnetoencefalografía, así como problemas de estimación relacionados a las imágenes de resonancia magnética por tensor de difusión. Se analizan los efectos de variaciones en el modelo de cabeza utilizado, en el posicionamiento de los electrodos y la modelización de la actividad cerebral de fondo. Se estudia también la influencia del ruido propio del sistema de adquisición en imágenes de tensor de difusión y mediciones derivadas de éste. Tales influencias se plasman en errores en la estimación de la conductividad eléctrica, necesaria para la adecuada modelización de la cabeza, así como en la estimación de la geometría estructural intracerebral, denominada tractografía. 


\section{Agradecimientos}

A las instituciones que financiaron la realización de esta tesis: el Consejo Nacional de Investigaciones Científicas y Técnicas y la Universidad Nacional de La Plata. Al Laboratorio de Electrónica Industrial Control e Instrumentación (LEICI) por proporcionar el lugar y material de trabajo.

Al Ing. Alejandro Blenkmann y a la Dra. Silvia Kochen, del Centro de Epilepsia del Hospital Ramos Mejía y el Instituto de Biología Celular y Neurociencias "Prof. E. de Robertis" de la UBA, por proporcionar registros de electroencefalografía e imágenes de todas las modalidades de pacientes reales.

Al Dr. Agustín Ibáñez del el Laboratorio de Psicología Experimental y Neurociencias del Instituto de Neurología Cognitiva por proporcionar señales reales de potenciales evocados.

A todo el grupo de Procesamiento Estadístico de Señales del LEICI, por tanto intercambio de mates, charlas e ideas.

A Patricia Gauzellino, por el constante apoyo y confianza.

A mis directores Carlos Muravchik y Nicolás von Ellenrieder, a quienes no sólo agradezco la dirección de mi doctorado, sino también la confianza, el apoyo y la amistad brindadas desde un principio.

A mi familia por el apoyo incondicional.

Y por sobre todas las personas, a Malén, quién cada día me muestra el hermoso sentido de la vida. 


\section{Índice general}

1. Introducción 1

1.1. Resultados preexistentes . . . . . . . . . . . . . . 3

1.2. Contribuciones originales . . . . . . . . . . . . . . 7

1.3. Organización de la tesis . . . . . . . . . . . . . . . . 9

2. El problema directo en E/MEG 11

2.1. Introducción . . . . . . . . . . . . . . . . . . . . . 11

2.2. Planteo matemático del problema directo en E/MEG . . . . . . . . 13

2.2.1. Aproximación cuasiestática de las ecuaciones de

Maxwell . . . . . . . . . . . . . . . . . . . 14

2.2.2. Formulación diferencial . . . . . . . . . . . . . . . 15

2.2.3. Modelo de fuente dipolar . . . . . . . . . . . . . . . 17

2.3. Resolución numérica mediante el MEF . . . . . . . . . . . . . . . 18

2.3.1. Formulación Variacional . . . . . . . . . . . . . . 18

2.3.2. Existencia y Unicidad . . . . . . . . . . . . . . . 19

2.3.3. Discretización del problema directo en E/MEG . . . . . . . 21

2.3.4. Coordenadas de volumen y selección del subespacio vectorial $V_{h} 22$

2.3.5. Resolución del problema directo . . . . . . . . . . . . . . 25

2.3.6. Medidas de desempeño . . . . . . . . . . . . . . . 27

2.4. Simulaciones y comparación de resultados . . . . . . . . . . . 28

2.4.1. Resultados en EEG . . . . . . . . . . . . . . . . . 29 
2.4.2. Resultados en MEG . . . . . . . . . . . . . . . 32

2.5. Conclusiones . . . . . . . . . . . . . . . . 36

3. Influencia del modelo de cabeza en el problema inverso de EEG 39

3.1. Introducción . . . . . . . . . . . . . . . . . . . . . . . . . 39

3.2. Materiales y Métodos . . . . . . . . . . . . . . . . . . . . . 42

3.2.1. Participantes .................. . . 43

3.2.2. Estímulos de potenciales evocados . . . . . . . . . . . 43

3.2.3. Adquisición de datos . . . . . . . . . . . . . . . . . . 44

3.2.4. Modelo de cabeza y problema directo . . . . . . . . . . . . . 44

3.2.5. Localización de fuentes . . . . . . . . . . . . . . . . . 46

3.2.6. Comparación de los modelos de cabeza . . . . . . . . . . 48

3.2.7. Selección de datos . . . . . . . . . . . . . . . . . . . . 48

3.3. Resultados . . . . . . . . . . . . . . . . 51

3.3.1. Comparación de modelos de cabeza . . . . . . . . . . . . 51

3.3.2. Localización de fuentes . . . . . . . . . . . . . . . . . . . . 52

3.4. Discusión y conclusiones . . . . . . . . . . . . . . . . . 58

4. Efecto del modelo de cabeza en ECoG y E/MEG 63

4.1. Introducción . . . . . . . . . . . . . . . . . . . . . 63

4.2. Métodos . . . . . . . . . . . . . . . . 66

4.2.1. Modelo de cabeza . . . . . . . . . . . . . . . 67

4.2.2. Problema directo . . . . . . . . . . . . . . . . . . . 68

4.2.3. Cuantificación de la influencia del modelo . . . . . . . . . . . 69

4.3. Resultados . . . . . . . . . . . . . . . . . . . . . 72

4.3.1. Factores de desempeño . . . . . . . . . . . . . . 73

4.3.2. Variaciones de la calidad de señal . . . . . . . . . . . 83

4.4. Discusión y conclusiones . . . . . . . . . . . . . . . . . 85

5. Influencia de la incertidumbre en la posición de los electrodos $\quad 91$ 
5.1. Introducción . . . . . . . . . . . . . . . . . . . . . . 91

5.2. Descripción del modelo aleatorio . . . . . . . . . . . . . . . . 93

5.3. Cotas de desempeño . . . . . . . . . . . . . . . . . . . . 96

5.4. Discretización . . . . . . . . . . . . . . . . 97

5.5. Resultados . . . . . . . . . . . . . . . . . . . . 99

5.6. Conclusiones . . . . . . . . . . . . . . . . . 106

6. Estimación por constricción de la matriz de covarianza 109

6.1. Introducción . . . . . . . . . . . . . . . . . . . . 109

6.2. Estimadores usuales . . . . . . . . . . . . . . . . . . 112

6.2.1. Modelo de señal . . . . . . . . . . . . . . . . . . . . . . 112

6.2.2. Covarianza muestral y de máxima verosimilitud . . . . . . . 113

6.3. Estimadores de constricción . . . . . . . . . . . . . . . . . . . 114

6.3.1. Selección de la estructura subyacente . . . . . . . . . . . 116

6.3.2. Esquemas propuestos e implementación numérica . . . . . . . 119

6.3.3. Cotas de desempeño . . . . . . . . . . . . . . . . 120

6.4. Resultados . . . . . . . . . . . . . . . . . . . . 121

6.4.1. Resultados utilizando datos sintéticos . . . . . . . . . . 122

6.4.2. Resultados utilizando datos reales . . . . . . . . . . . . 127

6.5. Discusión y conclusiones . . . . . . . . . . . . . . . 130

7. Desempeño de los estimadores de constricción en beamforming 133

7.1. Introducción . . . . . . . . . . . . . . . . . . . 133

7.2. Métodos . . . . . . . . . . . . . . . . . 135

7.2.1. Beamforming de máximo contraste . . . . . . . . . 136

7.2.2. Beamforming por constricción . . . . . . . . . . . 138

7.3. Resultados . . . . . . . . . . . . . . . . . . 138

7.4. Discusión y conclusiones . . . . . . . . . . . . . . . . . . . 141

8. Influencia del ruido en imágenes de tensor de difusión 145 
8.1. Introducción . . . . . . . . . . . . . . . . . . . . . . 145

8.2. Métodos . . . . . . . . . . . . . . . . . . . . . 148

8.2.1. Modelo de señal . . . . . . . . . . . . . . . . . . 148

8.2.2. Cota de Cramér-Rao . . . . . . . . . . . . . . 151

8.3. Resultados . . . . . . . . . . . . . . . . . 156

8.3.1. Resultados utilizando datos sintéticos . . . . . . . . . . 156

8.3.2. Resultados utilizando datos reales . . . . . . . . . . . . 164

8.4. Discusión y Conclusiones . . . . . . . . . . . . . . . . . . . 171

9. Conclusiones

$\begin{array}{ll}\text { A. Deducción de algunas fórmulas del Capítulo } 2 & 179\end{array}$

A.1. Demostración del Teorema 2.3.1 . . . . . . . . . . . . . . . . . . . 179

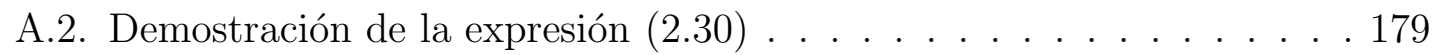

B. Deducción de algunas fórmulas del Capítulo $3 \quad 181$

B.1. Breve reseña sobre sLORETA . . . . . . . . . . . . . . . . . . . 181

C. Deducción de algunas fórmulas del Capítulo $5 \quad 183$

C.1. Gradiente de potencial . . . . . . . . . . . . . . . . 183

C.2. Problema directo del gradiente . . . . . . . . . . . . . . . . 184

C.3. Deducción de (5.12) . . . . . . . . . . . . . . . . 186

$\begin{array}{ll}\text { D. Deducción de algunas fórmulas del Capítulo } 6 & 187\end{array}$

D.1. Demostración del teorema 6.3 .1 . . . . . . . . . . . . . . 187

D.2. Demostración del teorema $6.3 .2 \ldots$. . . . . . . . . . . . . 188

D.3. Obtención de la cota de Cramér-Rao . . . . . . . . . . . . . . . . 189

E. Deducción de algunas fórmulas del Capítulo $8 \quad 191$

E.1. Cálculo de $\boldsymbol{J}$. . . . . . . . . . . . . . . . . . . . . . . . . . . . . . 191

E.2. Cálculo de las derivadas de los autovalores respecto a $\boldsymbol{\theta}$. . . . . . 193 


\section{Lista de abreviaturas}

BEM: Boundary elements method - Método de elementos de contorno.

CRB: Cramér-Rao bound - Cota de Cramér-Rao.

EAR: Ellipsoidal area ratio - Relación de área elipsoidal.

ECoG: Electrocorticografía.

EEG: Electroencefalografía.

E/MEG: Electro-magnetoencefalografía.

ECM: Error cuadrático medio.

FA: Fractional anisotropy - Anisotropía fraccional.

LCR: Líquido céfalorraquídeo.

MEF: Método de elementos finitos.

MAG: Error de magnitud

MEG: Magnetoencefalografía.

MLE: Maximum Likelihood Estimator - Estimador de máxima verosimilitud.

MCB: Maximum contrast beamformer - Beamformer de máximo contraste.

NRDM: Normalized RDM - Medida de diferencia relativa normalizada.

PD: Problema directo.

PE: Potenciales evocados.

PI: Problema inverso.

RA: Relative anisotropy - Anisotropía relativa.

RDM: Relative difference measure - Medida de diferencia relativa.

RM: Resonancia magnética.

RMp: Resonancia magnética paralela.

RM-TD: Resonancia magnética por tensor de difusión.

ROI: Region of interest - Región de interés.

SNR: Signal to noise ratio - Relación señal a ruido.

TAC: Tomografía axial computarizada. 


\section{Capítulo 1}

\section{Introducción}

A lo largo de la historia el hombre ha desarrollado la ciencia como herramienta para indagar la naturaleza en todas sus áreas. Paradójicamente el funcionamiento del órgano que le permite desarrollar estos conocimientos, el cerebro, es uno de los misterios de la naturaleza que hasta hace poco no se habían podido develar. Esta situación comenzó a cambiar en las últimas décadas, con la comprensión de la bioquímica celular y el funcionamiento de las neuronas. Sin embargo, el funcionamiento conjunto de millones de neuronas de un cerebro trabajando aún no se comprende cabalmente. Para estudiar el cerebro en funcionamiento se han desarrollado, a lo largo del siglo pasado, algunas herramientas entre las que podemos contar a la electroencefalografía, la magnetoencefalografía y la resonancia magnética. La electroencefalografía y magnetoencefalografía son técnicas no invasivas que permiten el estudio del funcionamiento del cerebro con una elevada resolución temporal, del mismo orden que los procesos químicos que dan origen a la actividad eléctrica de las neuronas. La resonancia magnética, por otro lado, es un método no invasivo que permite caracterizar adecuadamente la anatomía interna del encéfalo con gran resolución espacial.

Desde el punto de vista electromagnético el conjunto de neuronas activas del cerebro puede describirse como una distribución de densidad de corriente eléctrica. Las ecuaciones de Maxwell permiten calcular la distribución de potencial eléctrico generada por dichas neuronas, y de esta manera pueden predecirse, si la fuente 
es conocida, las diferencias de potencial que se medirán entre distintos puntos en la superficie de la cabeza. De igual manera se puede calcular el campo magnético que generan las corrientes presentes en los tejidos, y así predecir las mediciones que se obtendrían en sensores de campo magnético situados fuera de la cabeza. Esta predicción de las mediciones o cálculo del potencial o campo magnético a partir de una fuente de actividad eléctrica conocida, es el llamado problema directo de la electro/magnetoencefalografía. Partiendo de mediciones de potencial y campo magnético es posible, bajo ciertas hipótesis, estimar parámetros de la fuente de actividad neuronal, tales como su ubicación e intensidad. Esto se conoce como problema inverso de electro/magnetoencefalografía. Dado que en las mediciones siempre hay ruido presente, este es un problema que debe resolverse con herramientas de procesamiento estadístico de señales. El problema inverso no tiene en general una única solución, por lo que deben formularse una serie de suposiciones para lograr una adecuada caracterización de la fuente de actividad. Estas suposiciones normalmente se realizan al formular modelos de la fuente neuronal de actividad eléctrica y del medio en que tiene lugar la actividad electromagnética.

Existen muchos aspectos del problema que afectan la solución de los problemas directo e inverso. Entre ellos se pueden mencionar los modelos adoptados para la fuente de actividad eléctrica, para la geometría de la cabeza y las características electromagnéticas de los tejidos, así como el modelo del ruido que afecta las mediciones en el problema inverso.

Esta tesis estudia distintos aspectos de los problemas directo e inverso de la electro/magnetoencefalografía, con particular énfasis en el estudio de la influencia del modelo de cabeza utilizado, la incertidumbre que produce el desconocimiento de la posición exacta de los electrodos de medición, la actividad cerebral de fondo y la adecuada modelización de conductividad eléctrica de la cabeza.

Desde un punto de vista general los análisis presentados en esta tesis permiten determinar en que aspectos se debe enfatizar el estudio o la calidad de los modelos para lograr la precisión requerida en neurología en la solución de los problemas relacionados con la electro/magnetoencefalografía. 


\subsection{Resultados preexistentes}

La existencia de fenómenos electromagnéticos en el cuerpo humano se conoce hace tiempo, y ha sido estudiada en detalle (Titomir y Kneppo, 1994; Malmivuo y Plonsey, 1995). En particular, el estudio de la relación entre la actividad eléctrica generada por fuentes en el cerebro y el potencial eléctrico medido sobre el cuero cabelludo se presentó en Geselowitz (1967), y la generación de campo magnético por fuentes en el cerebro en Geselowitz (1970). Desde entonces se trabajó en el tema y existe una gran cantidad de resultados relacionados. Una buena recopilación de los mismos puede encontrarse en Hämäläinen et al. (1993); Hallez et al. (2007).

Para el cálculo de la solución del problema directo (PD) de la electro/magnetoencefalografía (E/MEG), es decir la predicción de la distribución de potencial y campo magnético generado por una fuente conocida, existen diversos métodos, tanto analíticos para geometrías simples (Sarvas, 1987; de Munck y Peters, 1993; Zhang, 1995), como numéricos para geometrías realistas (van den Broek et al., 1996; Vanrumste et al., 2001; Zhang et al., 2004; Wolters et al., 2006). El problema inverso (PI) de E/MEG, o la estimación de parámetros de una fuente desconocida a partir de mediciones contaminadas por ruido, también se estudió en profundidad. Existe un gran número de algoritmos para resolverlo (Mosher y Leahy, 1998; Sekihara et al., 1999; Dogandžić y Nehorai, 2000; de Munck et al., 2002; Pascual-Marqui, 2002; Sekihara y Nagarajan, 2007), así como cotas generales en el desempeño que se puede lograr en su resolución (Muravchik y Nehorai, 2001; von Ellenrieder et al., 2006).

Uno de los factores que determinan el desempeño que puede obtenerse en el PI de E/MEG es el modelo de la cabeza utilizado, existiendo numerosos trabajos que estudian distintos aspectos del tema (van den Broek et al., 1998; Awada et al., 1998; Huiskamp et al., 1999; Vanrumste et al., 2000). Dado que para una geometría con simetría esférica existe una solución analítica (de Munck y Peters, 1993), mientras que para geometrías realistas se deben emplear métodos numéricos, existe un gran número de trabajos en los que se analiza la diferencia entre la solución del PI para un modelo esférico y un modelo realista (Meijs et al., 1988; Cuffin, 1990; Roth et al., 1993; Yvert et al., 1997; Baillet et al., 2001; Vatta et al., 2010). Estos trabajos 
recurren a simulaciones reiterativas con la finalidad de comparar la reconstrucción de fuentes dipolares utilizando cada modelo. Sin embargo, en dichos trabajos se consideran los errores en la reconstrucción de fuentes dipolares debidas sólo a variaciones en la geometría de la cabeza, sin tener en cuenta factores influyentes como la conductividad eléctrica. Muchos trabajos (Ramon et al., 2006; Wolters et al., 2006; Rullmann et al., 2009; Güllmar et al., 2010) han mostrado la influencia de la incorporación de esta información en la resolución del PI en E/MEG, concluyendo que es de importancia. En esta tesis se determina el error en la solución del PI al usar un modelo genérico completamente realista, comparando la solución con la obtenida al usar modelos más sencillos.

El modelo de cabeza puede distar aún más de los esféricos en situaciones en las que se consideren variaciones geométricas debidas a intervenciones quirúrgicas y/o utilización de instrumental de medición invasivo. Técnicas como la electrocorticografía (ECoG) y la electroencefalografía de profundidad (sEEG) son ejemplo de ello (Lachaux et al., 2003; Blount et al., 2008). Éstas son utilizadas en situaciones en las que la determinación precisa de la fuente de actividad es de suma importancia, como lo es en estudios prequirúrgicos realizados sobre pacientes candidatos a la resección de áreas de corteza por funcionamiento anómalo. Estos estudios suelen durar de siete a diez días (Tao et al., 2007b; Huiskamp et al., 2010), por lo que muchos investigadores se valen de ello para analizar el alcance de los resultados de E/MEG en comparación a ECoG y sEEG. Con esta finalidad se realizan mediciones simultáneas de E/MEG y ECoG, a partir de las que se extraen múltiples conclusiones (Mikuni et al., 1997; Pacia y Ebersole, 1997; Oishi et al., 2002; Tao et al., 2005, 2007a; Ray et al., 2007; Hashiguchi et al., 2007; van der Loo et al., 2007; Yamazaki et al., 2011). Sin embargo, en estos trabajos no se considera de forma adecuada la variación del modelo provista por la intervención quirúrgica y la implantación del dispositivo de medición, llevando a resultados controversiales. En la presente tesis se muestra la influencia del modelo en estos casos, permitiendo evaluar la incidencia de su simplificación en los resultados y conclusiones extraídas.

Otra fuente de error en la resolución del PI de EEG corresponde a la incertidumbre en la localización de los electrodos. Existen diversos métodos de adquisición 
de dichas posiciones, basados en reiteradas mediciones de distancias entre electrodos (de Munck et al., 1991), en imágenes de resonancia magnética (Wang et al., 1996; Yoo et al., 1997; de Munck et al., 2012), en fotografías digitales (von Ellenrieder et al., 2009a) o utilizando lápices posicionadores electromagnéticos (San Martín, 2006). Dichos mecanismos carecen de exactitud en la reconstrucción de la posición de los electrodos debido a la incertidumbre inherente a todo sistema de adquisición. Es por ello que en diferentes trabajos se estudia la influencia de estos errores en la localización de las fuentes de actividad cerebral (Kavanagh et al., 1978; Towle et al., 1993; Khosla et al., 1999; Hoey et al., 2000; Bruno et al., 2004; Wang et al., 2006). En dichos trabajos se considera el análisis del desempeño logrado a partir de la utilización de métodos de resolución del PI particulares, llevando a la conclusión de que los resultados serán válidos sólo cuando ese método de resolución sea considerado. Por ello, en este trabajo se plantea un método general que permite cuantificar la influencia de la incertidumbre en la posición de los electrodos sea cual fuere el método de resolución del PI escogido.

Otro aspecto a tener en cuenta en el análisis del PI de EEG es la adecuada modelización y consideración de la actividad cerebral de fondo. La influencia de este ruido ha sido estudiada con detalle en numerosos trabajos (Sekihara et al., 1994; Lütkenhöner, 1998; Dogandžić y Nehorai, 2000; de Munck et al., 2002; Huizenga et al., 2002; Waldorp et al., 2002). Debido a la naturaleza del problema se ha mostrado que resulta factible la consideración de independencia entre las variaciones temporales y espaciales de dicha actividad, resultando en una simplificación del problema general de caracterización espacio-temporal (de Munck et al., 2002; Huizenga et al., 2002; Bijma et al., 2005). Luego, modelos paramétricos matemática, física y fisiológicamente plausibles surgen en pos de la caracterización de la variación espacial (de Munck et al., 1992, 1997; Huizenga et al., 2002) y temporal (Bijma et al., 2003). Sin embargo, aún no ha sido estudiada en detalle la aplicación de este conocimiento en la resolución del PI, el cual resulta de suma utilidad en situaciones en las que el número de realizaciones de señal impide el uso de los estimadores comúnmente utilizados. En este trabajo se introduce una técnica de estimación que saca provecho de este conocimiento, plasmando su ventaja en la mejora de la resolución del PI. 
La adecuada consideración de la actividad cerebral de fondo resulta fundamental y de primer orden frente a otras fuentes de ruido (von Ellenrieder, 2005). Esto se plasma en la utilización directa de la matriz de covarianza por parte de numerosos algoritmos (de Munck et al., 2002; Huizenga et al., 2002), resultando ser el método de filtrado espacial o beamforming uno de ellos (van Veen et al., 1997; Chen et al., 2006; Sekihara y Nagarajan, 2007). La aplicación de esta técnica en E/MEG resulta un tanto restrictiva debido a la necesidad de considerar mediciones de señal en diferentes condiciones, denominadas de modo activo y control (Chen et al., 2006; Sekihara y Nagarajan, 2007). Estas condiciones no siempre se dan en la práctica clínica, existiendo situaciones en donde el número de realizaciones de señal es pequeño. Por esta razón se introduce un método basado en la caracterización adecuada del ruido de fondo de EEG, el cual permite obtener beneficios en la estimación de la fuente de actividad cerebral aún conociendo un escaso número de realizaciones.

Finalmente, cabe destacar que para obtener modelos realistas se necesita de información proveniente de distintas modalidades de imágenes biomédicas, tales como la resonancia magnética por tensor de difusión (RM-TD) (Basser et al., 1994). Básicamente, esta técnica consiste en la medición de la difusividad de las moléculas de agua a lo largo de múltiples direcciones con la finalidad de estudiar la movilidad de los protones de hidrógeno en función de la orientación. Dado que la difusión en los tejidos no es libre, el agua se difundirá más rápidamente en la dirección alineada con la estructura interna y más lentamente en la dirección perpendicular a membrana que lo contiene, permitiendo la obtención de información acerca de su estructura anatómica. Luego, la RM-TD permite analizar tanto la anatomía in vivo del cerebro (Mori et al., 1999; Basser et al., 2000; Lazar et al., 2003), como así también estimar la conductividad eléctrica de los tejidos blandos (Tuch et al., 2001; Güllmar et al., 2010). Diversos estudios analizan la influencia de los errores de medición en la estimación del campo tensorial de difusión (Lazar y Alexander, 2003; Alexander, 1997; Caan et al., 2010). Sin embargo, dichos estudios no consideran la incorporación de sistemas de múltiples bobinas receptoras (Roemer et al., 1990; Pruessmann et al., 1999) y las ventajas que éstos proveen en la estimación de parámetros relacionados al tensor de difusión y, consecuentemente, al mapa tensorial que representa la con- 
ductividad eléctrica del cerebro. Esto conllevó a la realización de un análisis genérico que permite vislumbrar las ventajas de estos sistemas en la estimación del mapa de difusión cerebral independientemente del algoritmo de estimación empleado.

\subsection{Contribuciones originales}

Las contribuciones originales de esta tesis están relacionadas con el análisis de la influencia de incertidumbres en relación a los problemas directo e inverso de E/MEG, así como en la reconstrucción del mapa tensorial dado por imágenes de RM-TD.

Se planteó un método de resolución del PD en E/MEG mediante el método de los elementos finitos, considerando funciones de base lineales. Esto permitió el adecuado modelado de la cabeza, considerando tanto la geometría realista como la adecuada conductividad eléctrica. La formulación planteada permitió llegar a expresiones analíticas de fácil implementación numérica. Asimismo, se presentó el marco teórico que fundamenta la existencia, unicidad y tasa de convergencia de la solución bajo ciertas hipótesis. Parte de los resultados se presentaron en Beltrachini et al. (2008).

Se estudió el efecto del modelo de cabeza utilizado en circunstancias en las que no es posible contar con modelos personalizados de los individuos. En esos casos, diversos modelos son empleados, siendo el más utilizado el esférico con capas de conductividad eléctrica isótropa. Con el fin de reducir las fuentes de error se realizó un modelo realista basado en atlas que permitió considerar una adecuada geometría y conductividad eléctrica del medio. Utilizando el método de los elementos finitos desarrollado y una técnica de resolución del PI se comparó la incertidumbre debida a cada modelo, concluyendo en la necesidad de la utilización del modelo realista. Los resultados se presentaron en (Beltrachini et al., 2011a,b). Asimismo, diferentes aplicaciones del modelo realista fueron presentadas en Fernández-Corazza et al. (2011) y publicadas en Ibáñez et al. (2011b,c); von Ellenrieder et al. (2012).

Se analizó el efecto de la modelización adecuada en mediciones conjuntas de ECoG y E/MEG. Esto permitió cuantificar debidamente la influencia del dispositivo de adquisición invasivo y las variaciones del modelo debidas a la intervención 
quirúrgica en las mediciones realizadas. Los resultados son de sumo interés en la práctica clínica, especialmente en estudios tendientes a caracterizar, de forma experimental, los límites de las técnicas no invasivas. Se mostraron ventajas de MEG frente a EEG en dichas situaciones. Los resultados presentados en esta tesis aún no han sido publicados.

Se estudió también el efecto de incertezas de pequeña magnitud en el posicionamiento de los electrodos de EEG mediante un análisis por perturbaciones. Estas incertezas de pequeña magnitud representan errores en las técnicas de adquisición de dichas posiciones, tales como lápices posicionadores electromagnéticos o fotografías digitales. El análisis realizado permitió determinar que, en situaciones generales, el efecto de las perturbaciones es de importancia secundaria en el PI de EEG, frente a otras causas de error. Los resultados se publicaron en Beltrachini et al. (2011c).

Se estudió la caracterización espacio-temporal del ruido de fondo y analizó su influencia en el PI en EEG. Dicha caracterización es de vital importancia en relación a la resolución del PI, así como en relación al estudio de la actividad de fondo per se. Se propuso el método de estimación por constricción y presentaron sus ventajas teórico-prácticas cuando el número de realizaciones disponible es escaso. Los resultados se presentaron en (Beltrachini et al., 2009, 2010) y publicaron en Beltrachini et al. (2011e).

Se desarrolló un método de resolución del PI en EEG basado en un método de beamforming existente y los estimadores por constricción de la covarianza del ruido de fondo. Esto permitió la adecuada consideración de dicha actividad, la cual resulta ser sumamente influyente si no se caracteriza adecuadamente. Se mostró la validez del método en circunstancias en las que sólo pocas realizaciones de señal se conocen. Los resultados presentados en esta tesis aún no han sido publicados.

Otro aporte incluido en esta tesis es la determinación de los efectos del ruido de medición en factores derivados de la RM-TD. Dichos estudios son de gran importancia para la caracterización in vivo de tejidos blandos, utilizados tanto en la investigación como en la práctica clínica. Asimismo, se analizó la influencia de la utilización de sistemas adquisidores de múltiples bobinas, cuantificando sus ventajas. Algunos de los resultados se presentaron en Beltrachini et al. (2011d). 


\subsection{Organización de la tesis}

El Capítulo 2 constituye una introducción al PD en E/MEG. Se presenta una formulación matemática del problema directo y los métodos utilizados normalmente para resolverlo, haciendo especial hincapié en el método de elementos finitos, por ser éste el que permite el adecuado tratamiento de la conductividad eléctrica del medio. Asimismo, se realiza un estudio teórico-práctico que permite validar su utilización.

En el Capítulo 3 se analiza la influencia del modelo de cabeza utilizado en la resolución del PI en E/MEG. Se pone especial énfasis en situaciones en las que los modelos individuales no se encuentran disponibles, lo cual es cotidiano en problemas de localización de fuentes relacionadas a potenciales evocados. Se describe una metodología que permite caracterizar dicho efecto y se utiliza en la localización del potencial evocado N170.

En el cuarto capítulo se analiza la influencia del modelo en estudios de ECoG y E/MEG simultáneos. Se presenta una metodología para la obtención del modelo y presentan resultados que permiten caracterizar su incidencia en las conclusiones extraídas de dichos estudios.

En el quinto capítulo se estudia el efecto de variaciones de pequeña magnitud en la posición de los electrodos en el PI de EEG. Se cuantifica el efecto esperable en la localización de fuentes de actividad cerebral mediante la cota de Cramér-Rao y se presentan resultados obtenidos con la misma.

En el sexto capítulo se analiza el problema de caracterización del ruido de fondo en EEG. Se presenta el método de estimación por constricción, el cual permite una adecuada estimación del momento de segundo orden de dicho ruido, aún cuando se posean escasas realizaciones del proceso. Se muestran las ventajas de este método frente a los estimadores convencionales, utilizando datos sintéticos y reales.

El Capítulo 7 presenta el método de filtrado espacial por constricción para la resolución del PI en EEG, el cual constituye una aplicación directa de los estimadores homónimos presentados en el capítulo anterior. Se muestran las ventajas de dichos estimadores, conllevando a una formulación menos restrictiva que las usualmente consideradas. 
En el octavo capítulo se estudian los efectos del ruido de medición en imágenes de RM-TD. Se propone un modelo matemático que permite considerar la influencia de la adquisición de señal utilizando múltiples bobinas receptoras. Utilizando la cota de Cramér-Rao se cuantifica el efecto de dicho ruido en la estimación de la difusión del agua en el cerebro y diversos factores derivados de ésta.

Por último en el Capítulo 9 se presentan las conclusiones generales de la tesis, así como posibles líneas de investigación a seguir.

Cabe destacar que los datos reales utilizados en los Capítulos 4 y 6 son producto del trabajo en conjunto con el Instituto de Biología Celular y Neurociencias "Prof. E. de Robertis" (IBCN), Facultad de Medicina, Universidad de Buenos Aires, y el Centro de Epilepsia, División Neurología, Hospital Ramos Mejía. Asimismo, los datos reales de potenciales evocados presentados en el Capítulo 3 corresponden al trabajo en conjunto con el Laboratorio de Psicología Experimental y Neurociencias del Instituto de Neurología Cognitiva (INECO). 


\section{Capítulo 2}

\section{El problema directo en E/MEG}

\subsection{Introducción}

La electro-magnetoencefalografía (E/MEG) es una técnica que consiste en medir el potencial eléctrico sobre la superficie de la cabeza (cuero cabelludo) y el campo magnético por fuera de ésta con la finalidad de detectar fuentes bioeléctricas internas. Constituye, junto con otras técnicas de análisis tales como la Resonancia Magnética por tensor de difusión (RM-DTI) y la Resonancia Magnética Funcional (RMf), la base de las neurociencias de carácter no invasivo. Suponiendo conocidas las fuentes de actividad cerebral y las características del medio conductor es posible obtener la distribución del potencial eléctrico y el campo magnético generado, planteo que lleva el nombre de Problema Directo (PD) en E/MEG. Pero el problema de interés clínico consiste justamente en lo contrario: conocida la distribución de potencial eléctrico y campo magnético a partir de mediciones en un conjunto discreto de puntos (i.e. sensores) buscar las fuentes de actividad bioeléctrica de interés con la mayor exactitud posible. Dicho problema, debido a su naturaleza no unívoca, es conocido como Problema Inverso (PI) en E/MEG. Dado que las mediciones se encuentran inevitablemente contaminadas por ruido, se utilizan técnicas estadísticas que, en conjunción con técnicas de resolución del PD, permiten su adecuado abordaje (Dale y Sereno, 1993). De allí se desprende la necesidad del estudio del PD en detalle dado que errores en su resolución se verán reflejados en la determinación de las fuentes 
que originan un conjunto de mediciones.

El PD se encuentra totalmente ligado a los modelos utilizados en la caracterización tanto del medio como de las fuentes de actividad. En la literatura se proponen diferentes modelos de cabeza, dependiendo del nivel de detalle que se pretende del estudio. Estos modelos pueden ser tan sencillos como la consideración de geometrías esféricas con conductividad eléctrica isótropa (de Munck, 1988; Ermer et al., 2001), aumentando la complejidad hasta considerar geometrías y mapas de conductividad eléctrica realistas (Wolters et al., 2006). Si bien los modelos simples son muy utilizados en la bibliografía, las consideraciones realizadas sobre los mismos conllevan a errores inevitables en la resolución del PI (Vanrumste et al., 2002; von Ellenrieder et al., 2006; Güllmar et al., 2010; Vatta et al., 2010), limitando la precisión de los resultados. Luego, el adecuado modelado de la cabeza humana resulta de vital interés estudios de neurociencias relacionados a mediciones de E/MEG.

Múltiples métodos surgen para la resolución del PD considerando modelos realistas, tal como el de elementos de contorno (Brebbia y Domínguez, 1992), el sin mallas (von Ellenrieder et al., 2005b) y el de diferencias finitas (Vanrumste et al., 2001; Hallez et al., 2007). Sin embargo, no todos logran realizar un balance adecuado en la resolución del PD. Mientras que los dos primeros no posibilitan el modelado realista de la conductividad eléctrica (solo de forma isótropa), el tercero impone una discretización regular del dominio (Hallez et al., 2007), la cual se muestra en este capítulo que no siempre es recomendable. Por esta razón el método de los elementos finitos (MEF) surge como método apto para lidiar convenientemente con la situación. Existen numerosos trabajos que estudian la factibilidad del MEF en la aplicación al problema directo en E/MEG (Zhang et al., 2004; Wolters et al., 2006, 2007; Güllmar et al., 2010). Sin embargo la implementación propuesta en la literatura puede resultar un tanto engorrosa y/o computacionalmente costosa, por lo que no siempre es sencilla su utilización. Por esta razón se presenta la resolución del PD utilizando el MEF y funciones de base lineales, las cuales permiten llegar a formulaciones de fácil implementación. Se acompaña al estudio con el tratamiento teórico correspondiente que permite asegurar la existencia y unicidad de la solución, así como tasas de convergencia de acuerdo a la discretización de dominio considerada. 
El presente capítulo se organiza del siguiente modo: en la Sección 2.2 se obtiene una caracterización matemática del PD en E/MEG, validando algunas simplificaciones propias del mismo. Asimismo, se plantean las bases matemáticas del PD, concluyendo en la obtención de una ecuación diferencial con condiciones de borde de Neumann cuya solución determina la distribución de potencial eléctrico sobre el volumen conductor. En la Sección 2.3 se reformula el problema diferencial como un problema variacional para luego demostrar existencia y unicidad de la solución analítica bajo ciertas hipótesis. Luego se desarrolla el MEF de primer orden, considerado el más adecuado al problema de interés por permitir buena adaptación de la malla al volumen real sin aumentar en gran forma el número de incógnitas. En la Sección 2.4 se comparan los resultados obtenidos en la sección precedente tanto para medios isótropos como anisótropos. Por último, en la Sección 2.5 se extraen conclusiones concernientes a los resultados obtenidos y se presentan líneas futuras de trabajo.

\subsection{Planteo matemático del problema directo en E/MEG}

En esta sección se presenta la formulación matemática que caracteriza al PD en E/MEG. En principio, se justifican simplificaciones válidas propias del modelo del medio bajo estudio, las cuales permiten una más sencilla manipulación de las ecuaciones de Maxwell que gobiernan a los fenómenos electromagnéticos. Luego se obtiene la formulación diferencial asociada al potencial eléctrico y campo magnético. Por último, se muestra una breve reseña sobre los modelos de fuentes de corriente adecuados al problema. Planteos similares pueden verse, por ejemplo, en Hämäläinen et al. (1993); Hallez et al. (2007). 


\subsubsection{Aproximación cuasiestática de las ecuaciones de Maxwell}

Suponiendo conocida la conductividad eléctrica de la cabeza y las fuentes que generan la actividad neurobiológica, son las ecuaciones de Maxwell, junto con la de continuidad, las que permiten realizar un análisis cuantitativo de la situación. Considerando que en dicho volumen la permeabilidad magnética es constante e igual a la del vacío, las ecuaciones que representan al fenómeno en el punto $\boldsymbol{r}$ del espacio y en el tiempo $t$ vienen dadas por (Jackson, 1975)

$$
\begin{aligned}
\nabla \cdot \boldsymbol{E}(\boldsymbol{r} ; t) & =\frac{\rho(\boldsymbol{r} ; t)}{\epsilon_{0}} \\
\nabla \times \boldsymbol{E}(\boldsymbol{r} ; t) & =-\frac{\partial \boldsymbol{B}(\boldsymbol{r} ; t)}{\partial t}, \\
\nabla \cdot \boldsymbol{B}(\boldsymbol{r} ; t) & =0 \\
\nabla \times \boldsymbol{B}(\boldsymbol{r} ; t) & =\mu_{0}\left(\boldsymbol{J}(\boldsymbol{r} ; t)+\epsilon_{0} \frac{\partial \boldsymbol{E}(\boldsymbol{r} ; t)}{\partial t}\right), \\
\boldsymbol{J}(\boldsymbol{r} ; t) & =\boldsymbol{\sigma}(\boldsymbol{r}) \boldsymbol{E}(\boldsymbol{r} ; t)+\frac{\partial \boldsymbol{P}(\boldsymbol{r} ; t)}{\partial t},
\end{aligned}
$$

en donde $\boldsymbol{E}(\boldsymbol{r} ; t)$ y $\boldsymbol{B}(\boldsymbol{r} ; t)$ son los campos eléctrico y magnético, respectivamente, $\boldsymbol{P}(\boldsymbol{r} ; t)=\left(\epsilon(\boldsymbol{r})-\epsilon_{0}\right) \boldsymbol{E}(\boldsymbol{r} ; t)$ es la polarización, $\epsilon(\boldsymbol{r})$ es la permitividad del material, $\epsilon_{0}$ es la permitividad del vacío, $\mu_{0}$ es la permeabilidad magnética del vacío, y $\boldsymbol{\sigma}(\boldsymbol{r})$ la conductividad del medio. Cabe destacar que numerosos estudios muestran que la conductividad $\boldsymbol{\sigma}(\boldsymbol{r})$ dentro de la cabeza carece tanto de homogeneidad (Yan et al., 1991) como de isotropía (Kim et al., 2003), por lo que se la considera como una magnitud tensorial.

Se busca en lo siguiente justificar que es posible despreciar los términos de campo eléctrico y magnético generados a partir de variaciones de los mismos en el tiempo, aproximación que lleva el nombre de cuasiestática. De (2.4) y (2.5) se tiene que

$$
\boldsymbol{\nabla} \times \boldsymbol{B}(\boldsymbol{r} ; t)=\mu_{0}\left(\boldsymbol{\sigma}(\boldsymbol{r}) \boldsymbol{E}(\boldsymbol{r} ; t)+\epsilon(\boldsymbol{r}) \frac{\partial \boldsymbol{E}(\boldsymbol{r} ; t)}{\partial t}\right) .
$$

Sabiendo que $\boldsymbol{E}(\boldsymbol{r} ; t)=\boldsymbol{E}_{0}(\boldsymbol{r}) e^{j 2 \pi f t}$, considerar la aproximación cuasiestática en (2.6) equivale a pedir $|\epsilon(\boldsymbol{r}) \partial \boldsymbol{E}(\boldsymbol{r} ; t) / \partial t| \ll|\boldsymbol{\sigma}(\boldsymbol{r}) \boldsymbol{E}(\boldsymbol{r} ; t)|$, o bien $|j 2 \pi \epsilon(\boldsymbol{r}) f \boldsymbol{E}(\boldsymbol{r} ; t)| \ll$ 
$|\boldsymbol{\sigma}(\boldsymbol{r}) \boldsymbol{E}(\boldsymbol{r} ; t)|$ lo cual se cumple para los valores típicos de los tejidos involucrados $\epsilon=10^{5} \epsilon_{0}, f \simeq 100 \mathrm{~Hz}$ y $\boldsymbol{\sigma}=0,3 \boldsymbol{I}_{3} \Omega^{-1} m^{-1}$, con $\boldsymbol{I}_{3}$ la matriz identidad de $3 \times 3$. Luego, $\nabla \times \boldsymbol{B}(\boldsymbol{r}) \simeq \mu_{0} \boldsymbol{\sigma}(\boldsymbol{r}) \boldsymbol{E}(\boldsymbol{r})$.

Por otro lado se busca justificar la aproximación cuasiestática en la expresión (2.2), para lo cual se toma el rotor de la misma

$$
\boldsymbol{\nabla} \times \boldsymbol{\nabla} \times \mathbf{E}=-\frac{\partial(\boldsymbol{\nabla} \times \mathbf{B})}{\partial t}=-j 2 \pi f \mu_{0}\left(\boldsymbol{\sigma}+j 2 \pi f \epsilon \boldsymbol{I}_{3}\right) \boldsymbol{E} .
$$

Considerando los valores típicos dados anteriormente, la solución de esta ecuación diferencial tiene una constante de variación espacial dada por

$$
\lambda=\left|2 \pi f \mu_{0}(\sigma+j 2 \pi f \epsilon)\right|^{-1 / 2}=65 m
$$

en donde $\sigma=0,3 \Omega^{-1} m^{-1}$. Como el volumen bajo estudio es mucho menor que esta medida, se puede validar la aproximación cuasiestática para la expresión (2.7). Luego, como resulta $\boldsymbol{\nabla} \times \boldsymbol{E}(\boldsymbol{r})=\mathbf{0}$, el campo eléctrico puede ser expresado mediante su función potencial eléctrico $\boldsymbol{E}(\boldsymbol{r})=-\boldsymbol{\nabla} u(\boldsymbol{r})$.

\subsubsection{Formulación diferencial}

Es de utilidad diferenciar la densidad de corriente producida por la actividad neuronal en dos componentes: la primera debida a la activación sincronizada y local de una población, denominada corriente primaria (Hämäläinen et al., 1993; Malmivuo y Plonsey, 1995), la cual se designa mediante $\boldsymbol{J}^{p}(\boldsymbol{r})$. La segunda comprende las corrientes que permiten cerrar el circuito eléctrico, llamada corriente de volumen o retorno, y dada por $\boldsymbol{J}^{v}(\boldsymbol{r})=\boldsymbol{\sigma}(\boldsymbol{r}) \boldsymbol{E}(\boldsymbol{r})$. El resultado total será la densidad de corriente neta en la cabeza,

$$
\boldsymbol{J}(\boldsymbol{r})=\boldsymbol{J}^{p}(\boldsymbol{r})+\boldsymbol{\sigma}(\boldsymbol{r}) \boldsymbol{E}(\boldsymbol{r})
$$

La ecuación anterior ilustra la importancia de la corriente primaria en la vecindad de un grupo activo de células debida a la actividad cerebral, así como la pasividad de la corriente de volumen sobre todo el medio. Por ende, localizar la corriente 
primaria equivale a encontrar la fuente de actividad cerebral. Si se reemplaza $\boldsymbol{E}(\boldsymbol{r})$ por $-\boldsymbol{\nabla} u(\boldsymbol{r})$ en (2.8) y luego se toma la divergencia de la misma, se obtiene

$$
\boldsymbol{\nabla} \cdot \boldsymbol{J}(\boldsymbol{r})=\boldsymbol{\nabla} \cdot \boldsymbol{J}^{p}(\boldsymbol{r})-\boldsymbol{\nabla} \cdot(\boldsymbol{\sigma}(\boldsymbol{r}) \boldsymbol{\nabla} u(\boldsymbol{r})) .
$$

Utilizando la aproximación cuasiestática sobre (2.4), se tiene que $\boldsymbol{\nabla} \cdot \boldsymbol{J}(\boldsymbol{r})=0$, por lo que el problema directo en EEG consiste en encontrar el potencial eléctrico $u(\boldsymbol{r})$ tal que satisfaga

$$
\left\{\begin{array}{rl}
\boldsymbol{\nabla} \cdot \boldsymbol{J}^{p}(\boldsymbol{r})=\boldsymbol{\nabla} \cdot(\boldsymbol{\sigma}(\boldsymbol{r}) \boldsymbol{\nabla} u(\boldsymbol{r})) & \text { en } \Omega \\
(\boldsymbol{\sigma}(\boldsymbol{r}) \boldsymbol{\nabla} u(\boldsymbol{r})) \cdot \check{\boldsymbol{n}}=\mathbf{0} & \text { en } \partial \Omega
\end{array},\right.
$$

en donde $\Omega$ es el volumen de interés (i.e. la cabeza), $\partial \Omega$ es la superficie del mismo volumen (i.e. la superficie exterior del cuero cabelludo), y la segunda ecuación implica que en la superficie de la cabeza la componente de la densidad de corriente normal a ésta es nula.

Una vez resuelto el problema directo en EEG se puede proceder al problema directo en MEG. En efecto, de (2.3) se desprende que existe una función vectorial $\boldsymbol{A}(\boldsymbol{r})$ que satisface $\boldsymbol{B}(\boldsymbol{r})=\boldsymbol{\nabla} \times \boldsymbol{A}(\boldsymbol{r})$, llamada función potencial vectorial. Reemplazando en (2.4) se tiene

$$
\boldsymbol{\nabla} \times(\boldsymbol{\nabla} \times \boldsymbol{A}(\boldsymbol{r}))=\boldsymbol{\nabla}(\boldsymbol{\nabla} \cdot \boldsymbol{A}(\boldsymbol{r}))-\nabla^{2} \boldsymbol{A}(\boldsymbol{r})=\mu_{0} \boldsymbol{J}(\boldsymbol{r}),
$$

en donde $\nabla^{2}$ es el operador Laplaciano vectorial ${ }^{1}$. Considerando la condición de calibre de Coulomb, $\boldsymbol{\nabla} \cdot \boldsymbol{A}=0$, por lo que el problema a resolver resulta ser

$$
\nabla^{2} \boldsymbol{A}(\boldsymbol{r})=-\mu_{0} \boldsymbol{J}(\boldsymbol{r})
$$

Esta es nuevamente una ecuación de Poisson en donde, a diferencia del caso anterior, cambia la condición de borde. Como la permeabilidad magnética de la cabeza se consideró igual a la del aire, no existe otra opción que asumir que el campo magnético debido a la fuente de actividad cerebral es nulo en el infinito, $\boldsymbol{B}(\boldsymbol{r}) \rightarrow 0$ con $|\boldsymbol{r}| \rightarrow \infty$. Este problema puede resolverse analíticamente (Jackson, 1975), siendo su solución

$$
\boldsymbol{A}(\boldsymbol{r})=\frac{\mu_{0}}{4 \pi} \int_{\Omega} \frac{\boldsymbol{J}\left(\boldsymbol{r}^{\prime}\right)}{\left\|\boldsymbol{r}-\boldsymbol{r}^{\prime}\right\|} d \boldsymbol{r}^{\prime}
$$

\footnotetext{
${ }^{1} \nabla^{2} \boldsymbol{A}=\left(\boldsymbol{\nabla}^{2} A_{x}, \nabla^{2} A_{y}, \nabla^{2} A_{z}\right)$.
} 
Luego, el campo magnético en $\boldsymbol{r}$ vendrá dado por

$$
\boldsymbol{B}(\boldsymbol{r})=\nabla \times \boldsymbol{A}(\boldsymbol{r})=\frac{\mu_{0}}{4 \pi} \int_{\Omega} \frac{\boldsymbol{J}\left(\boldsymbol{r}^{\prime}\right) \times\left(\boldsymbol{r}-\boldsymbol{r}^{\prime}\right)}{\left\|\boldsymbol{r}-\boldsymbol{r}^{\prime}\right\|^{3}} d \boldsymbol{r}^{\prime} .
$$

Por último, reemplazando (2.8) en (2.10) se obtiene

$$
\boldsymbol{B}(\boldsymbol{r})=\boldsymbol{B}_{0}(\boldsymbol{r})-\boldsymbol{B}_{s}(\boldsymbol{r})
$$

en donde

$$
\boldsymbol{B}_{0}(\boldsymbol{r})=\frac{\mu_{0}}{4 \pi} \int_{\Omega} \frac{\boldsymbol{J}^{p}\left(\boldsymbol{r}^{\prime}\right) \times\left(\boldsymbol{r}-\boldsymbol{r}^{\prime}\right)}{\left\|\boldsymbol{r}-\boldsymbol{r}^{\prime}\right\|^{3}} d \boldsymbol{r}^{\prime},
$$

es el campo magnético primario debido a la corriente de la fuente, y

$$
\boldsymbol{B}_{s}(\boldsymbol{r})=\frac{\mu_{0}}{4 \pi} \int_{\Omega} \frac{\boldsymbol{\sigma}\left(\boldsymbol{r}^{\prime}\right) \boldsymbol{\nabla} u\left(\boldsymbol{r}^{\prime}\right) \times\left(\boldsymbol{r}-\boldsymbol{r}^{\prime}\right)}{\left\|\boldsymbol{r}-\boldsymbol{r}^{\prime}\right\|^{3}} d \boldsymbol{r}^{\prime}
$$

es el campo magnético secundario debido a las corrientes de volumen.

Dado que el campo magnético es una magnitud vectorial, en lo siguiente resulta de interés el cálculo de la proyección del mismo en una dirección determinada $\check{\boldsymbol{n}}_{l}$. Esta dirección queda determinada en algunos dispositivos de medición de campo, como los conformados bobinas conductoras, en donde $\check{\boldsymbol{n}}_{l}$ no es más que la dirección de su eje. En general se tiene

$$
\boldsymbol{B}(\boldsymbol{r}) \cdot \check{\boldsymbol{n}}_{l}=\boldsymbol{B}_{0}(\boldsymbol{r}) \cdot \check{\boldsymbol{n}}_{l}-\boldsymbol{B}_{s}(\boldsymbol{r}) \cdot \check{\boldsymbol{n}}_{l}
$$

en donde $\boldsymbol{B}_{0}(\boldsymbol{r}) \cdot \check{\boldsymbol{n}}_{l}$ se calcula fácilmente fijando un modelo de fuente. Para calcular la proyección del campo secundario se reemplaza $\boldsymbol{B}_{s}(\boldsymbol{r})$ en el segundo término de (2.14) por la expresión (2.13). Utilizando la propiedad $\boldsymbol{a} \cdot(\boldsymbol{b} \times \boldsymbol{c})=\boldsymbol{b} \cdot(\boldsymbol{c} \times \boldsymbol{a})$ se llega a

$$
\boldsymbol{B}_{s}(\boldsymbol{r}) \cdot \check{\boldsymbol{n}}_{l}=\frac{\mu_{0}}{4 \pi} \int_{\Omega} \boldsymbol{\sigma}\left(\boldsymbol{r}^{\prime}\right) \boldsymbol{\nabla} u\left(\boldsymbol{r}^{\prime}\right) \cdot \gamma_{l}\left(\boldsymbol{r}, \boldsymbol{r}^{\prime}\right) d \boldsymbol{r}^{\prime},
$$

en donde $\gamma_{l}\left(\boldsymbol{r}, \boldsymbol{r}^{\prime}\right)=\frac{\left(\boldsymbol{r}-\boldsymbol{r}^{\prime}\right)}{\left\|\boldsymbol{r}-\boldsymbol{r}^{\prime}\right\|^{3}} \times \check{\boldsymbol{n}}_{l}$.

\subsubsection{Modelo de fuente dipolar}

En problemas como el descripto es necesario contar con un modelo que represente a la fuente de actividad cerebral $\boldsymbol{J}^{p}(\boldsymbol{r})$. En el caso de suponer que los focos de 
actividad cerebral son lo suficientemente concentrados, se puede utilizar un modelo de múltiples dipolos (von Ellenrieder et al., 2009c), debido tanto a su simplicidad y a que ha probado ser buena aproximación en numerosas aplicaciones (de Munck et al., 1988a), tal como epilepsia (Merlet y Gotman, 1999; Kobayashi et al., 2005), potenciales evocados (Shibata et al., 2002) o actividad espontánea de fondo (de Munck et al., 1992). En el caso más focal o concentrado se considera, sin pérdida de generalidad, un único dipolo ubicado en $\boldsymbol{r}_{0}$ y con momento dipolar $\boldsymbol{Q}$ que mejor represente a la corriente primaria en un solo punto. Para este caso en particular se tendrá que la corriente primaria está dada por

$$
\boldsymbol{J}^{p}(\boldsymbol{r})=\boldsymbol{Q} \delta\left(\boldsymbol{r}-\boldsymbol{r}_{0}\right)
$$

Otros modelos de fuente más realistas existen en la bibliografía (Yetik et al., 2005, 2006), pero en el presente análisis se omiten por simplicidad en la explicación y desarrollos.

\subsection{Resolución numérica mediante el MEF}

En la presente sección se plantea la resolución del problema directo en E/MEG. En principio se determina una formulación variacional que permite relajar las condiciones impuestas sobre la solución buscada. Luego, se realiza un breve estudio sobre la existencia y unicidad de la solución y las condiciones que lo permiten. Tras mostrar la discretización del problema directo en E/MEG, se presenta la transformación de volumen, utilizada en el planteo final del método de resolución propuesto.

\subsubsection{Formulación Variacional}

Tal como se destacó en la sección anterior, el problema directo en EEG consiste en encontrar la función escalar $u(\boldsymbol{r})$ que satisfaga (2.9). Se ve claramente que este sistema requiere de una solución $u \in \mathcal{C}^{2}(\Omega)$ (conjunto de funciones dos veces diferenciables con derivada segunda continua), lo cual vuelve al método un tanto restrictivo. Es con la finalidad de relajar las condiciones sobre la solución que se replantea el 
problema y se busca resolver (2.9) en el sentido de promedios pesados (Becker et al., 1981). Es decir, se busca la solución $u(\boldsymbol{r})$ que satisfaga

$$
\int_{\Omega} v(\boldsymbol{r}) \boldsymbol{\nabla} \cdot \boldsymbol{J}^{p}(\boldsymbol{r}) d \boldsymbol{r}-\int_{\Omega} v(\boldsymbol{r}) \boldsymbol{\nabla} \cdot(\boldsymbol{\sigma}(\boldsymbol{r}) \boldsymbol{\nabla} u(\boldsymbol{r})) d \boldsymbol{r}+\int_{\partial \Omega} v(\boldsymbol{r})(\boldsymbol{\sigma}(\boldsymbol{r}) \boldsymbol{\nabla} u(\boldsymbol{r})) \cdot \check{\boldsymbol{n}} d \boldsymbol{r}=0,
$$

para todas las funciones $v(\boldsymbol{r})$ pertenecientes a un espacio de funciones adecuado. Este espacio de funciones de peso (también llamadas funciones de prueba) tiene que ser lo suficientemente conveniente para que (2.17) tenga sentido. Es por ello que se escoge al espacio de Hilbert-Sobolev $H^{1}(\Omega)$ compuesto por todas las funciones cuadrado-integrables cuyas derivadas de primer orden también son cuadradointegrables, i.e. $H^{1}(\Omega)=\left\{v: v, \partial v / \partial x_{i} \in L^{2}(\Omega)\right\}$, con norma asociada dada por $\|v(\boldsymbol{r})\|_{H^{1}(\Omega)}=\left\{\int_{\Omega}\left(|v(\boldsymbol{r})|^{2}+|\boldsymbol{\nabla} v(\boldsymbol{r})|^{2}\right) d \boldsymbol{r}\right\}^{1 / 2}$ (Carey y Oden, 1983). Luego, haciendo uso del Teorema de la Divergencia sobre la ecuación (2.17) se llega a

$$
\int_{\Omega} \boldsymbol{\nabla} v(\boldsymbol{r})^{T} \boldsymbol{\sigma}(\boldsymbol{r}) \boldsymbol{\nabla} u(\boldsymbol{r}) d \boldsymbol{r}=-\int_{\Omega} v(\boldsymbol{r}) \boldsymbol{\nabla} \cdot \boldsymbol{J}^{p}(\boldsymbol{r}) d \boldsymbol{r}
$$

en donde se utilizó la igualdad $\boldsymbol{\nabla} \cdot(a \boldsymbol{b})=\boldsymbol{\nabla} a \cdot \boldsymbol{b}+a \boldsymbol{\nabla} \cdot \boldsymbol{b}$. Esta expresión muestra un importante relajamiento de las condiciones impuestas sobre $u(\boldsymbol{r})$ : mientras que en (2.9) se necesitaba $u(\boldsymbol{r}) \in \mathcal{C}^{2}(\Omega)$, en (2.18) sólo se necesita que $u(\boldsymbol{r}) \in \mathcal{C}^{1}(\Omega)$ (conjunto de funciones diferenciables con derivada continua). Es por ello que ésta última formulación lleva el nombre de formulación débil o variacional.

\subsubsection{Existencia y Unicidad}

Considerando la formulación débil dada en (2.18) es consecuente su planteo lineal, utilizando formas lineales y bilineales. Es decir, la formulación variacional dada por la ecuación (2.9) se puede expresar de la forma

$$
a(u, v)=\mathcal{L}(v)
$$

en donde

$$
\mathcal{L}(v)=-\int_{\Omega} v(\boldsymbol{r}) \boldsymbol{\nabla} \cdot \boldsymbol{J}^{p}(\boldsymbol{r}) d \boldsymbol{r}
$$

es una forma lineal y

$$
a(u, v)=\int_{\Omega} \boldsymbol{\nabla} v^{T}(\boldsymbol{r}) \boldsymbol{\sigma}(\boldsymbol{r}) \boldsymbol{\nabla} u(\boldsymbol{r}) d \boldsymbol{r}
$$


es una forma bilineal. Para mostrar la existencia y unicidad de la solución es preciso realizar las siguientes definiciones y lemas asociados.

Definición 2.3.1 Una forma bilineal $B(u, v): V \times V \rightarrow K$ es simétrica en el espacio vectorial $V$ si cumple

- $B(u, v)=B(v, u), \forall u, v \in V$,

- $B(u+v, w)=B(u, w)+B(v, w), \forall u, v, w \in V$,

- $B(\lambda v, w)=\lambda B(v, w), \forall v, w \in V, \forall \lambda \in K$.

Lema 2.3.1 La forma bilineal $a(u, v)$ definida en $(2.21)$ es simétrica en $H^{1}(\Omega)$.

Su demostración es evidente de la definición y considerando $\boldsymbol{\sigma}$ simétrico, lo cual surge de su sentido físico.

Definición 2.3.2 Una forma bilineal $B(u, v)$ se dice continua en un espacio vectorial normado $V$ si y sólo si $\exists \gamma>0, \gamma \in K$, tal que $|B(u, v)| \leq \gamma\|u\|_{V}\|v\|_{V}$.

Lema 2.3.2 La forma bilineal $a(u, v)$ definida en (2.21) es continua en $H^{1}(\Omega)$ con $\gamma=\left|\lambda_{\text {máx }}\right|$, siendo $\lambda_{\text {máx }}$ el máximo autovalor del tensor $\boldsymbol{\sigma}$.

Para su demostración se remite a Wolters et al. (2007).

Definición 2.3.3 Una forma bilineal es V-elíptica (o elíptica sobre el espacio vectorial $V)$ si y sólo si $\exists \alpha>0, \alpha \in K$, tal que $a(v, v) \geq \alpha\|v\|_{V}^{2}, \forall v \in V$.

Lema 2.3.3 Sea $\lambda_{\text {min }}$ es el menor autovalor del tensor $\boldsymbol{\sigma}$ y s es la longitud de la arista de un cubo que contiene al dominio $\Omega$. Asimismo, sea $H_{*}^{1}(\Omega)$ el subespacio de $H^{1}(\Omega)$ dado por $H_{*}^{1}(\Omega)=\left\{v \in H^{1}(\Omega): \int_{\Omega} v(\boldsymbol{r}) d \boldsymbol{r}=0\right\}$. Luego, la forma bilineal $a(u, v)$ definida en (2.21) es $H_{*}^{1}$-elíptica con $\alpha=\frac{\lambda_{\min }}{1+4 s^{2}}$.

Para su demostración se remite a Wolters et al. (2007).

Definición 2.3.4 Una forma lineal $\mathcal{L}($.$) se dice continua sobre un espacio vectorial$ normado $V$ si y sólo si $\exists \Lambda>0, \Lambda \in \mathbb{R}$, tal que $|\mathcal{L}(v)| \leq \Lambda\|v\|_{V}, \forall v \in V$. 
Lema 2.3.4 Sea $\mathcal{L}(v) \in L^{2}(\Omega)$. Luego, la forma lineal $\mathcal{L}(v)$ definida en (2.20) es continua en $H^{1}(\Omega)$ con $\Lambda=\left\|\boldsymbol{\nabla} \cdot \boldsymbol{J}^{p}(\boldsymbol{r})\right\|_{L^{2}(\Omega)}$.

Su demostración se presenta en el Apéndice A.1.

Redefinido el problema, puede establecerse el siguiente Teorema de existencia y unicidad,

Teorema 2.3.1 Dado el problema definido por las ecuaciones (2.19)- (2.21), existe una única solución $u \in H_{*}^{1}(\Omega)$ que cumple la condición de estabilidad

$$
\|u(\boldsymbol{r})\|_{H_{*}^{1}(\Omega)} \leq \frac{\left\|\boldsymbol{\nabla} \cdot \boldsymbol{J}^{p}(\boldsymbol{r})\right\|_{L^{2}(\Omega)}\left(1+4 s^{2}\right)}{\lambda_{\min }} .
$$

Debe notarse que la validez del Teorema anterior se ve limitada (es decir, no es válido) para fuentes dipolares como las aquí propuestas. Sin embargo, la validez del mismo para fuentes cuadrado integrables sirve como incentivo hacia la búsqueda de espacios que permitan mostrar la existencia y unicidad de la solución para fuentes dipolares, así como marco para el planteo de modelos de fuente más realistas.

\subsubsection{Discretización del problema directo en E/MEG}

Sea ahora $V_{h}$ un subespacio de $H_{*}^{1}(\Omega)$ de dimensión finita $N$ y sea $\left\{\varphi_{i}\right\}_{i=1}^{N}$ una base de $V_{h}$ de modo tal que $\forall g \in V_{h}, g=\sum_{i=1}^{N} \eta_{i} \varphi_{i}, \eta_{i} \in \mathbb{R}$. Luego, el problema (2.19) discretizado pasará a ser el de buscar $u_{h} \in V_{h}$ que verifique

$$
a\left(u_{h}, v_{h}\right)=\mathcal{L}\left(v_{h}\right)
$$

para toda función $v_{h} \in V_{h}$. Tomando $v_{h}=\varphi_{j} \in V_{h} \mathrm{y} u_{h}=\sum_{i=1}^{N} \eta_{i} \varphi_{i}$, se tiene que

$$
\sum_{i=1}^{N} a\left(\varphi_{i}, \varphi_{j}\right) \eta_{i}=\mathcal{L}\left(\varphi_{j}\right)
$$

para todo $j=1, \ldots, N$. En forma matricial el sistema queda dado por

$$
\boldsymbol{A} \boldsymbol{\eta}=\boldsymbol{b}
$$

en donde $\boldsymbol{A}=\left\{a_{i j}\right\}_{i j} \in \mathbb{R}^{N \times N}$ con $a_{i j}=a\left(\varphi_{i}, \varphi_{j}\right)$ es llamada matriz de rigidez, $\boldsymbol{\eta}=\left\{\eta_{i}\right\}_{i} \in \mathbb{R}^{N}$ son los coeficientes buscados y $\boldsymbol{b}=\left\{b_{i}\right\}_{i} \in \mathbb{R}^{N}$ con $b_{i}=\mathcal{L}\left(\varphi_{i}\right)$ es 
llamado vector de carga. Por lo tanto, la resolución del sistema de ecuaciones (2.23) permite el cálculo de $u_{h}(\boldsymbol{r})$, la aproximación de la función de potencial eléctrico en $V_{h}$. Dado que $\boldsymbol{A}$ es positiva definida (ver Johnson (1987), Th.2.2, p.53), el sistema de ecuaciones (2.23) tiene una única solución. Basado en (2.22) y considerando $v_{h}=u_{h}$ en el Teorema 2.3 .1 puede verse que $\left\|u_{h}(\boldsymbol{r})\right\|_{H_{*}^{1}(\Omega)}$ presenta la misma cota que $\|u(\boldsymbol{r})\|_{H_{*}^{1}(\Omega)}$, lo cual constituye una base teórica que valida la adaptación del Teorema 2.3.1 aún sobre espacios de dimensión finita, asegurando la convergencia del método.

Para calcular el campo magnético se considera la proyección del mismo en la dirección de $\check{\boldsymbol{n}}_{l}$, como se expresa en (2.14). El término correspondiente al campo primario queda explícito una vez adoptado un modelo de fuente. Para la obtención del término correspondiente al campo secundario, se reemplaza $u_{h}(\boldsymbol{r})$ en (2.15). Notando que $\boldsymbol{\nabla} u_{h}\left(\boldsymbol{r}_{j}\right)=\sum_{i=1}^{N} \eta_{i} \boldsymbol{\nabla} \varphi_{i}\left(\boldsymbol{r}_{j}\right)$, se llega a

$$
\boldsymbol{B}_{s}(\boldsymbol{r}) \cdot \check{\boldsymbol{n}}_{l}=\sum_{i=1}^{N} s_{i}(\boldsymbol{r}) \eta_{i}=\boldsymbol{s}(\boldsymbol{r})^{T} \boldsymbol{\eta},
$$

en donde $\boldsymbol{s}(\boldsymbol{r})=\left\{s_{i}(\boldsymbol{r})\right\}_{i}$, con $s_{i}(\boldsymbol{r})=\frac{\mu_{0}}{4 \pi} \int_{\Omega} \boldsymbol{\sigma}\left(\boldsymbol{r}^{\prime}\right) \boldsymbol{\nabla} \varphi_{i}\left(\boldsymbol{r}^{\prime}\right) \cdot \gamma_{l}\left(\boldsymbol{r}, \boldsymbol{r}^{\prime}\right) d \boldsymbol{r}^{\prime}$.

\subsubsection{Coordenadas de volumen y selección del subespacio vectorial $V_{h}$}

En principio se busca calcular la matriz de rigidez $\boldsymbol{A}$ utilizada en (2.23). Para ello, en primer lugar se debe discretizar el dominio $\Omega$ en elementos finitos propiamente dichos. En este caso, debido a la amplia difusión y existencia de numerosos programas que lo permiten (Persson y Strang, 2004; Fang y Boas, 2009), se considera la discretización del dominio en tetraedros. Ésta permite la generación del subespacio $V_{h}$ a partir de la interpolación de las soluciones en los nodos que definen a los tetraedros del mallado.

Una vez discretizado el dominio, se presenta el método de transformación de coordenadas de volumen (Springer, 1946; Becker et al., 1981; Hutton, 2004; Zhang et al., 2004), utilizado en la Sección 2.3.5 para el consecuente planteo del problema directo en E/MEG. Para cada elemento del mallado se definen las coordenadas 


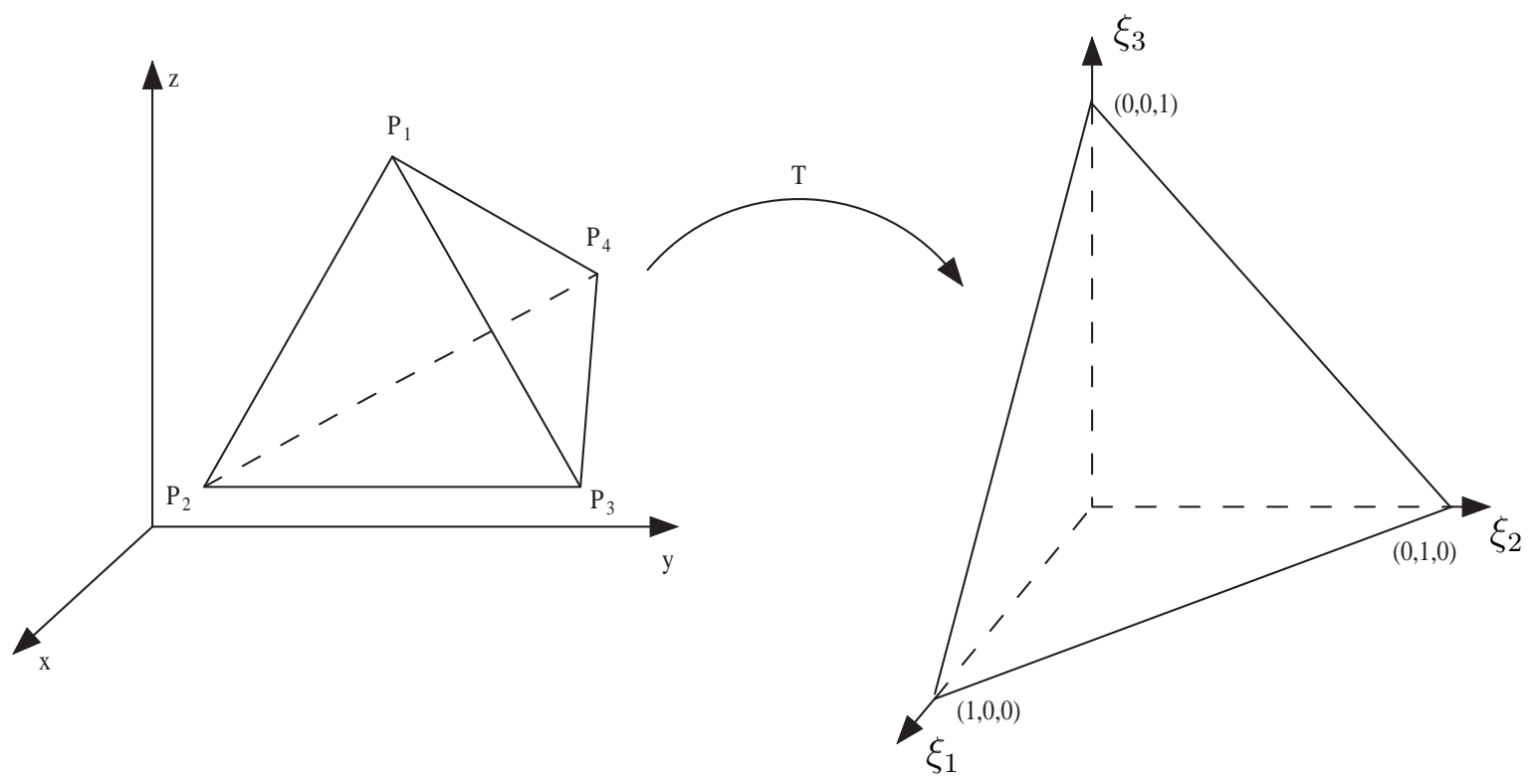

Figura 2.1: Representación gráfica de la transformación de volumen.

$\xi_{i}=V_{i} / V$, con $i=1,2,3,4$, en donde $V$ es el volumen del tetraedro y $V_{i}$ es el volumen de cada uno de los cuatro tetraedros conformados por tres vértices del tetraedro original y el punto interno del que se requieren las coordenadas. Debido a que $V=\sum_{k=1}^{4} V_{k}$, las coordenadas no serán linealmente independientes, por lo que en el análisis subsecuente se reemplazará a $\xi_{4}$ por $1-\xi_{1}-\xi_{2}-\xi_{3}$ cuando sea necesario. En cuanto al cambio de variables propuesto, tiene la particularidad de transformar cualquier tetraedro en uno ubicado en la posición canónica, tal como se muestra en la Figura 2.1.

Cabe destacar que conociendo los vértices del tetraedro, su volumen quedará expresado por

$$
V=\frac{1}{3 !}\left|\begin{array}{cccc}
1 & x_{1} & y_{1} & z_{1} \\
1 & x_{2} & y_{2} & z_{2} \\
1 & x_{3} & y_{3} & z_{3} \\
1 & x_{4} & y_{4} & z_{4}
\end{array}\right|
$$


y los volúmenes definidos para un punto $(x, y, z)$ interno por

$$
\begin{gathered}
V_{1}=\frac{1}{3 !}\left|\begin{array}{cccc}
1 & x & y & z \\
1 & x_{2} & y_{2} & z_{2} \\
1 & x_{3} & y_{3} & z_{3} \\
1 & x_{4} & y_{4} & z_{4}
\end{array}\right| ; \quad V_{2}=\frac{1}{3 !}\left|\begin{array}{cccc}
1 & x_{1} & y_{1} & z_{1} \\
1 & x & y & z \\
1 & x_{3} & y_{3} & z_{3} \\
1 & x_{4} & y_{4} & z_{4}
\end{array}\right| ; \\
V_{3}=\frac{1}{3 !}\left|\begin{array}{cccc}
1 & x_{1} & y_{1} & z_{1} \\
1 & x_{2} & y_{2} & z_{2} \\
1 & x & y & z \\
1 & x_{4} & y_{4} & z_{4}
\end{array}\right| ; \quad V_{4}=\frac{1}{3 !}\left|\begin{array}{cccc}
1 & x_{1} & y_{1} & z_{1} \\
1 & x_{2} & y_{2} & z_{2} \\
1 & x_{3} & y_{3} & z_{3} \\
1 & x & y & z
\end{array}\right| .
\end{gathered}
$$

Siguiendo la definición de la transformación dada anteriormente, se deduce que cada coordenada puede expresarse de la forma

$$
\xi_{i}=\frac{a_{i}+b_{i} x+c_{i} y+d_{i} z}{3 ! V}
$$

con $a_{i}, b_{i}, c_{i}$ y $d_{i}$ números reales. Por último, puede demostrarse que el determinante del Jacobiano de la transformación viene dado por $|J|=3$ ! $V$. Para más detalles sobre esta transformación se remite a Springer (1946) o la bibliografía anteriormente citada.

Se procede ahora a elegir el subespacio vectorial $V_{h}$ y una base $\mathcal{B}$ adecuada. Es por simplicidad de las mismas que se escoge una base del espacio de los polinomios en $(x, y, z)$ de hasta orden $n$ sobre el cuerpo de los reales, es decir, se toma $V_{h}=\mathcal{P}_{\mathbb{R}}^{(n)}$. Puede verse que los elementos de la base no serán más que sus coeficientes. Luego, se realiza la transformación de volumen, dejando como resultado una base $\mathcal{B}^{\prime}=\left\{\varphi_{i}\left(\xi_{1}, \xi_{2}, \xi_{3}, \xi_{4}\right)\right\}_{i=1}^{n}$, cuyos elementos no serán más que los coeficientes de los potenciales en los puntos de interés. Puede demostrarse (Silvester y Ferrari, 1994) que las funciones de forma en la base $\mathcal{B}^{\prime}$ serán

$$
\varphi_{i j k l}\left(\xi_{1}, \xi_{2}, \xi_{3}, \xi_{4}\right)=R_{i}\left(n_{0}, \xi_{1}\right) R_{j}\left(n_{0}, \xi_{2}\right) R_{k}\left(n_{0}, \xi_{3}\right) R_{l}\left(n_{0}, \xi_{4}\right),
$$

en donde $n_{0}=i+j+k+l$ es el grado del polinomio y

$$
R_{m}\left(n_{0}, \xi\right)=\frac{1}{m !} \prod_{k=0}^{m-1}\left(n_{0} \xi-k\right)
$$


En particular, si se considera $n=1, V_{h}=\mathcal{P}_{\mathbb{R}}^{(1)}$ y las funciones de base resultantes serán

$$
\varphi_{i}\left(\xi_{1}, \xi_{2}, \xi_{3}, \xi_{4}\right)=\xi_{i}, i=1,2,3,4
$$

las cuales permiten obtener un polinomio de interpolación de la forma

$$
u_{h}\left(\xi_{1}, \xi_{2}, \xi_{3}, \xi_{4}\right)=\xi_{1} u_{1}^{e}+\xi_{2} u_{2}^{e}+\xi_{3} u_{3}^{e}+\xi_{4} u_{4}^{e}
$$

en donde $u_{i}^{e}$ es el potencial en el nodo $P_{i}$ del tetraedro (numeración local del elemento correspondiente a una variable global $\eta_{j}$ determinada).

\subsubsection{Resolución del problema directo}

Una vez discretizado el dominio se procede a la obtención de las expresiones correspondientes para la resolución del PD en E/MEG. Se muestran las expresiones de las matrices de rigidez y vectores de carga elementales resultantes de la transformación de volumen para los casos de EEG y MEG, recalando en el caso particular de funciones de base lineales, las cuales permiten la obtención de expresiones cerradas.

\section{EEG}

Sea $\boldsymbol{r}$ un punto perteneciente al elemento $\Omega_{e}$. De acuerdo a lo desarrollado en la Sección 2.3.4 el potencial eléctrico en $\boldsymbol{r}$ dependerá de la resolución del sistema $\boldsymbol{A}_{e} \boldsymbol{\eta}_{e}=\boldsymbol{b}_{e}$, en donde el subíndice $e$ indica que se restringe el planteo a dicho elemento. Realizado el cambio de coordenadas de volumen, los elementos de la matriz de rigidez local $\boldsymbol{A}_{e}=\left\{a_{e}\left(\varphi_{i}, \varphi_{j}\right)\right\}_{i j}$ vienen dados por

$$
a_{e}\left(\varphi_{i}, \varphi_{j}\right)=\int_{0}^{1} \int_{0}^{1-\xi_{1}} \int_{0}^{1-\xi_{1}-\xi_{2}} g\left(\xi_{1}, \xi_{2}, \xi_{3}\right)|J| d \xi_{3} d \xi_{2} d \xi_{1}
$$

en donde

$$
g\left(\xi_{1}, \xi_{2}, \xi_{3}\right)=\frac{1}{(3 ! V)^{2}} \sum_{m=1}^{4} \sum_{n=1}^{4} K_{m n} \frac{\partial \varphi_{i}}{\partial \xi_{m}} \frac{\partial \varphi_{j}}{\partial \xi_{n}},
$$

y $K_{m n}$ son los elementos de la matriz $\boldsymbol{K}_{e}$ definida por

$$
\boldsymbol{K}_{e}=\boldsymbol{D}_{e}^{T} \boldsymbol{\sigma}_{e} \boldsymbol{D}_{e}
$$


con

$$
\boldsymbol{D}_{e}=\left(\begin{array}{cccc}
b_{1} & b_{2} & b_{3} & b_{4} \\
c_{1} & c_{2} & c_{3} & c_{4} \\
d_{1} & d_{2} & d_{3} & d_{4}
\end{array}\right)
$$

y $\boldsymbol{\sigma}_{e}$ es el tensor de conductividad eléctrica correspondiente al elemento (dentro del cual se supone constante). Debe notarse que tanto $\boldsymbol{\sigma}_{e}$ como $\boldsymbol{D}_{e}$ son independientes del punto del tetraedro $\boldsymbol{r}$, por lo que también lo será $\boldsymbol{K}_{e}$. Por ende, la ecuación (2.27) queda de la forma

$$
\begin{aligned}
a_{e}\left(\varphi_{i}, \varphi_{j}\right) & =\frac{1}{3 ! V} \int_{0}^{1} \int_{0}^{1-\xi_{1}} \int_{0}^{1-\xi_{1}-\xi_{2}} \sum_{m=1}^{4} \sum_{n=1}^{4} K_{m n} \frac{\partial \varphi_{i}}{\partial \xi_{m}} \frac{\partial \varphi_{j}}{\partial \xi_{n}} d \xi_{3} d \xi_{2} d \xi_{1} \\
& =\frac{1}{3 ! V} \sum_{m=1}^{4} \sum_{n=1}^{4} K_{m n} \int_{0}^{1} \int_{0}^{1-\xi_{1}} \int_{0}^{1-\xi_{1}-\xi_{2}} \frac{\partial \varphi_{i}}{\partial \xi_{m}} \frac{\partial \varphi_{j}}{\partial \xi_{n}} d \xi_{3} d \xi_{2} d \xi_{1}(2.29)
\end{aligned}
$$

En el caso especial de considerar funciones de base lineales, $\varphi_{i}=\xi_{i}$, por lo que $\partial \varphi_{i} / \partial \xi_{j}=\delta[i-j]$. Reemplazando en (2.29) se obtiene la matriz de rigidez elemental para funciones de forma lineales

$$
\boldsymbol{A}_{e}=\frac{1}{36 V} \boldsymbol{D}_{e}^{T} \boldsymbol{\sigma}_{e} \boldsymbol{D}_{e}
$$

Para calcular $\boldsymbol{b}_{e}$ se debe adoptar un modelo de fuente. En el caso de considerar el modelo de una única fuente dipolar, ubicada en $\boldsymbol{r}_{0}$ y con momento dipolar $\boldsymbol{Q}=\left(Q_{x}, Q_{y}, Q_{z}\right)$, utilizando la propiedad de extracción de la derivada de $\delta(\boldsymbol{r})$ se tiene que el vector de carga elemental $\boldsymbol{b}_{e}=\left\{\mathcal{L}\left(\varphi_{j}\right)\right\}_{j}$ viene dado por

$\mathcal{L}\left(\varphi_{j}\right)=-\int_{\Omega} \varphi_{j} \nabla \cdot\left(\boldsymbol{Q} \delta\left(\boldsymbol{r}-\boldsymbol{r}_{0}\right)\right) d \boldsymbol{r}=\left\{\begin{array}{ll}\left(Q_{x} b_{j}+Q_{y} c_{j}+Q_{z} d_{j}\right) /(3 ! V) & \text { si } \boldsymbol{r}_{0} \in \Omega_{e} \\ 0 & \text { en c.c. }\end{array}\right.$, en donde $b_{j}, c_{j}$ y $d_{j}$ son los definidos en la expresión (2.25).

Finalmente, se ensamblan todas las matrices y vectores elementales para el cálculo de la matriz de rigidez total $\boldsymbol{A}$ y el vector de carga total $\boldsymbol{b}$, como es usual en formulaciones de elementos finitos. Una vez acoplado, se calcula el potencial eléctrico mediante la resolución del sistema de ecuaciones (2.23). 


\section{MEG}

Una vez planteado el problema directo en EEG se procede al planteo correspondiente en MEG. Supóngase que se pretende calcular la proyección del campo magnético en la dirección $\check{\boldsymbol{n}}_{l}$ y en los puntos $\boldsymbol{r}_{j}, j=1, \ldots, N_{s}$, llamado $\boldsymbol{\vartheta}$. Éste contará con dos componentes, una debida al campo primario, $\boldsymbol{\vartheta}_{0}=\left\{\boldsymbol{B}_{0}\left(\boldsymbol{r}_{j}\right) \cdot \check{\boldsymbol{n}}_{l}\right\}_{j}$, y otra correspondiente al campo secundario, $\boldsymbol{\vartheta}_{s}=\left\{\boldsymbol{B}_{s}\left(\boldsymbol{r}_{j}\right) \cdot \check{\boldsymbol{n}}_{l}\right\}_{j}$. Asumiendo el modelo de fuente dipolar se ve fácilmente que

$$
\boldsymbol{\vartheta}_{0}=\frac{\mu_{0}}{4 \pi} \boldsymbol{Q} \cdot\left(\frac{\boldsymbol{r}_{j}-\boldsymbol{r}_{q}}{\left\|\boldsymbol{r}_{j}-\boldsymbol{r}_{q}\right\|^{3}} \times \check{\boldsymbol{n}}_{l}\right) .
$$

Por otro lado, en el Apéndice A.2 se muestra que

$$
\boldsymbol{\vartheta}_{s}=\sum_{e=1}^{N_{e}} \boldsymbol{S}_{e} \boldsymbol{\eta}_{e}
$$

en donde

$$
\boldsymbol{S}_{e}=\frac{\mu_{0}}{4 \pi 3 ! V_{e}} \boldsymbol{I}_{e} \boldsymbol{\sigma}_{e} \boldsymbol{D}_{e}
$$

es la matriz de rigidez elemental de dimensión $N_{s} \times 4$ e $\boldsymbol{I}_{e}$ es una matriz de $N_{s} \times 3$ con filas $\iota_{j}$ definidas por

$$
\boldsymbol{\iota}_{j}=\int_{\Omega_{e}} \boldsymbol{\gamma}_{l}\left(\boldsymbol{r}_{j}, \boldsymbol{r}^{\prime}\right) d \boldsymbol{r}^{\prime}
$$

$\mathrm{Al}$ igual que para el caso de EEG, se calcula la matriz de rigidez total $\boldsymbol{S}$, por lo que el problema directo de MEG quedará descripto por

$$
\vartheta=\boldsymbol{\vartheta}_{0}-\boldsymbol{S} \boldsymbol{\eta}
$$

en donde $\boldsymbol{\eta}$ es el potencial eléctrico en los nodos de interés.

\subsubsection{Medidas de desempeño}

Para determinar el error en la resolución del PD es usual la comparación de la solución obtenida numéricamente con la solución analítica sobre un dominio sencillo en donde ésta exista. Una medida de error muy utilizada (Schlitt et al., 1995) es la medida de diferencia relativa $(\mathrm{RDM})$. Esta medida de error se define por

$$
R D M_{k}=\frac{\left\|\boldsymbol{u}_{a}-\boldsymbol{u}_{r}\right\|_{k}}{\left\|\boldsymbol{u}_{r}\right\|_{k}}
$$


en donde $\boldsymbol{u}_{a}$ y $\boldsymbol{u}_{r}$ son los vectores de soluciones aproximadas y de referencia en los puntos de interés. Distintos valores de $k$ determinan diferencias en el parámetro a estudiar. En la presente tesis se considerarán dos de ellas, $k=2$ y $k=\infty$. En el primer caso, el $R D M_{2}$ indicará una suerte de promedio de error sobre todos los puntos de interés. Esta medida parece adecuada, por ejemplo, en estudios de E/MEG en donde se espera que los errores en las mediciones debidas al método de resolución sean aproximadamente del mismo orden. En el segundo caso, el $R D M_{\infty}$ indicará el máximo error normalizado cometido por la aproximación. Esta medida parece adecuada en ciertos estudios en donde la influencia de la aproximación no es de interés en todo el dominio, sino en un subconjunto de éste. Ejemplos de su aplicación se muestran a lo largo de la tesis.

En muchas ocasiones es deseable poder conocer distintos aspectos del error empleando más de una medida. Es usual en la literatura la utilización de la medida de diferencia relativa normalizada (NRDM) y el error en la magnitud de los vectores (MAG), propuestos en Meijs et al. (1989) y definidos por

$$
\begin{gathered}
N R D M_{k}=\left\|\frac{\boldsymbol{u}_{r}}{\left\|\boldsymbol{u}_{r}\right\|_{k}}-\frac{\boldsymbol{u}_{a}}{\left\|\boldsymbol{u}_{a}\right\|_{k}}\right\|_{k}, \\
M A G_{k}=\frac{\left\|\boldsymbol{u}_{a}\right\|_{k}}{\left\|\boldsymbol{u}_{r}\right\|_{k}} .
\end{gathered}
$$

Se ve claramente que, mientras que el NRDM considera errores en la distribución del vector de interés (pues sus medidas se encuentran normalizadas), el MAG hace lo propio con la magnitud o escala de los mismos, siendo ambos en conjunto tan informativos como el RDM definido anteriormente. Iguales consideraciones que en el caso del RDM se realizan sobre la elección de $k$.

\subsection{Simulaciones y comparación de resultados}

Una vez fundamentado el método elegido para el análisis, se llevó a la práctica y simuló en condiciones de interés. Para ello se consideró un dominio esférico de $8 \mathrm{~cm}$ de radio, similar al de la cabeza humana. Este dominio es de sumo interés en la comunidad científica por la disponibilidad de solución analítica considerando 
fuentes dipolares para el problema de EEG en forma general y MEG bajo ciertas suposiciones. Ejemplos de aplicación sobre geometrías realistas no esféricas se plantean en capítulos posteriores al presente. Para efectuar el estudio se generó una discretización de la esfera bajo análisis utilizando la aplicación ISO2Mesh ${ }^{2}$, la cual devuelve discretizaciones del dominio en tetraedros de alta calidad y con muy bajo costo computacional (Fang y Boas, 2009). En base a este modelo se realizaron simulaciones que permitieron evaluar variaciones de los factores de desempeño en función del modelo y su discretización.

Según el Lema 3.10 presentado en Wolters et al. (2007), el método de MEF ve limitada su estabilidad numérica a la resolución del mallado en las inmediaciones de la fuente y la distancia de ésta a zonas de salto de conductividad. En caso de considerar un modelo anisótropo, esto sucederá en cada elemento perteneciente a la discretización del cerebro, por lo que el mallado refinado en los alrededores de la fuente o potenciales zonas de ubicación resulta fundamental. Por esta razón es que se evaluaron diferencias en los resultados según se considere un mallado uniforme o uno adaptivo refinado en las inmediaciones de la fuente.

\subsubsection{Resultados en EEG}

En el PD de EEG es de sumo interés la validación sobre medios esféricos debido a la existencia de solución analítica aún en condiciones de anisotropía de la conductividad eléctrica (de Munck, 1988; Zhou y van Oosterom, 1992; de Munck y Peters, 1993). Esto permite validar el planteo formulado y evaluar los efectos de la variación de parámetros de interés, como la posición de la fuente, su orientación o la conductividad eléctrica.

En principio se procedió a calcular la variación del error (dados por $N R D M_{2}$ y $M A G_{2}$ ) en función del mallado considerado. Para ello se consideraron dos discretizaciones del dominio diferentes; la primera de ellas resultante de una discretización homogénea en donde la totalidad de los elementos presentan iguales dimensiones (aproximadamente); la segunda resultante de una discretización homogénea en el

\footnotetext{
${ }^{2}$ Disponible en http://iso2mesh.sourceforge.net/cgi-bin/index.cgi
} 

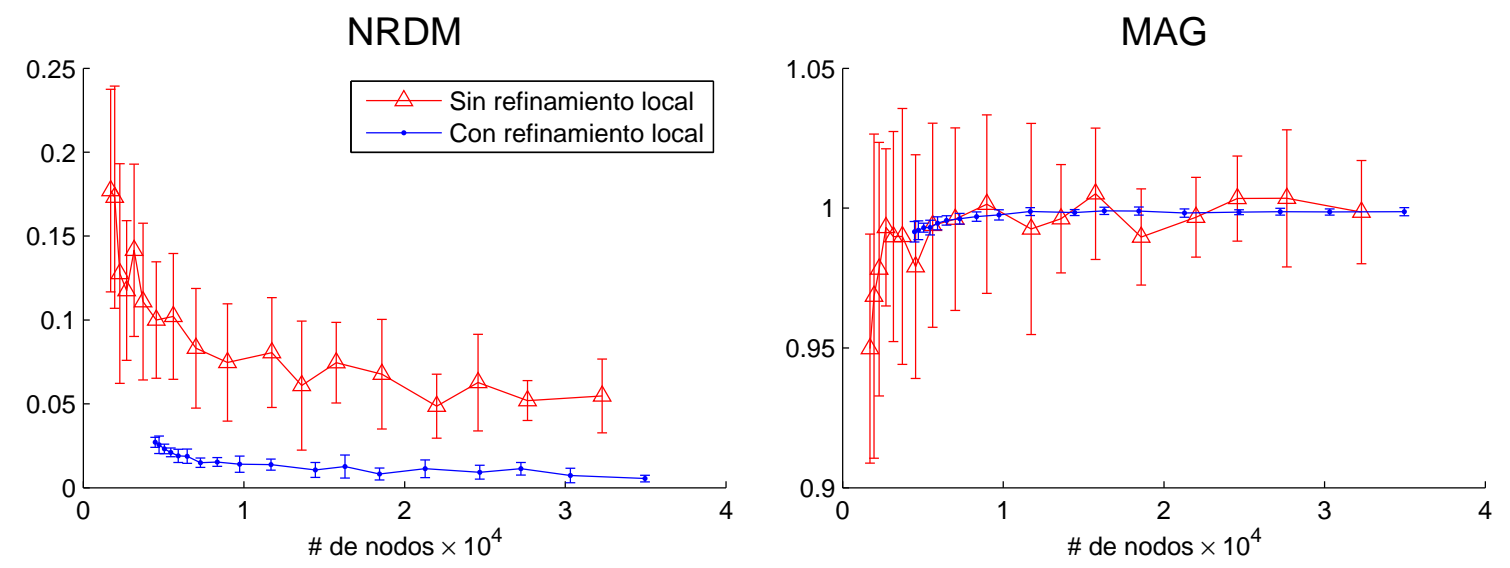

Figura 2.2: NRDM y MAG en función de la discretización, considerando el mallado homogéneo (rojo) y el refinado en inmediaciones de la fuente (azul). Se muestra la desviación estándar considerando cada modelo y 10 realizaciones del ensayo.

volumen, salvo en las inmediaciones de la fuente, en donde la discretización es más fina. Esta comparación surge en pos de validar la importancia de la resolución del mallado en las inmediaciones de la fuente, como se expresara anteriormente. En la Figura 2.2 se muestran los errores para cada modelo en función del número de nodos que integran el mallado. Se consideró una fuente dipolar posicionada a la mitad de la distancia entre el centro y el borde con orientación radial. Asimismo, la región de refinamiento se limitó a una esfera de $1 \mathrm{~mm}$ de radio en donde la longitud del elemento promedio se tomó igual a $0,1 \mathrm{~mm}$. Dado que los resultados son totalmente dependientes del mallado utilizado y de la posición relativa de la fuente en el mismo, se consideraron 10 realizaciones en las que tanto el mallado como la elevación y azimut de la fuente se variaron. La conductividad eléctrica se supuso isótropa.

Puede observarse la notable influencia del refinamiento local en la resolución del PD, visible tanto en el NRDM como en el MAG. Para igual número de nodos, el NRDM considerando refinamiento local decrece aproximadamente 10 veces respecto de no considerarlo, validando la importancia de su uso. Asimismo, y como era de esperarse, la desviación estándar resulta ser notablemente menor en caso de considerar el refinamiento, resultando en estimadores más consistentes.

En la Figura 2.3 se muestran los errores en el PD considerando fuentes dipolares 
con orientación radial (azul) y tangencial (rojo) en función de su distancia al centro de la esfera (también llamada excentricidad del dipolo), considerando conductividades eléctricas isótropas (i.e. con componente de la conductividad eléctrica radial $\sigma_{r}$ igual a la tangencial $\left.\sigma_{t}\right)$, anisótropa radial $\left(\sigma_{r}=10 \sigma_{t}\right)$ y anisótropa tangencial $\left(\sigma_{t}=10 \sigma_{r}\right)$. La discretización adoptada consistió en aproximadamente 6000 nodos (35000 elementos), con igual refinamiento local en las inmediaciones de la fuente que la considerada en la anterior figura. Puede apreciarse la diferencia de los errores cometidos según se considere isotropía, anisotropía radial o tangencial. Si bien la conductividad eléctrica de la cabeza no presenta una anisotropía tan marcada como las aquí presentadas, es de resaltar la influencia de la misma en la resolución del $\mathrm{PD}$, por lo que su consideración en el análisis es de primordial importancia. Asimismo, debe notarse que los mayores errores se producen bajo la consideración de conductividad eléctrica anisótropa tangencial, especialmente en fuentes profundas. Esto se debe a que la conductividad eléctrica no permite un aumento considerable del potencial eléctrico en el volumen, viéndose limitado el método por la precisión considerada en los cálculos.

Con el sólo fin de volver explícita la influencia de la adecuada consideración de la anisotropía del medio en el PD de EEG, se presenta en la Figura 2.4 la distribución del potencial eléctrico en el volumen considerando conductividad eléctrica isótropa (a.), anisótropa radial (b.) y anisótropa tangencial (c.). Dicho potencial se debe a una fuente dipolar con orientación tangencial y posicionada a 0,6 veces el radio de la esfera.

Puede verse la variabilidad de resultados según el mapa de conductividad eléctrica considerado, pudiendo resultar determinante en la interpretación de las mediciones de EEG. Debe notarse que, si bien los casos mostrados en la Figura 2.4 no corresponden completamente a los casos realistas, en donde la anisotropía de los tejidos dependen de la ubicación anatómica bajo análisis, permiten vislumbrar la necesidad de la inclusión de dicha información y, por ende, realza el valor del método aquí desarrollado. 
NRDM
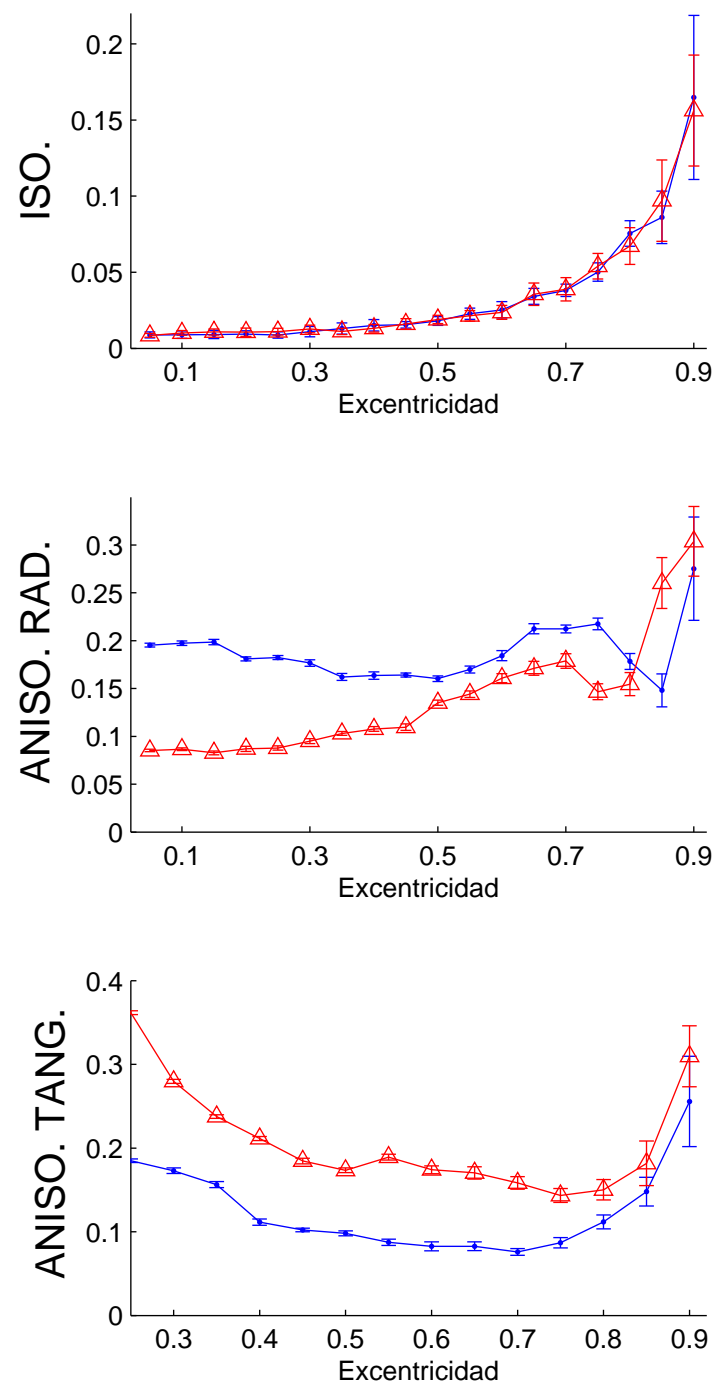

MAG
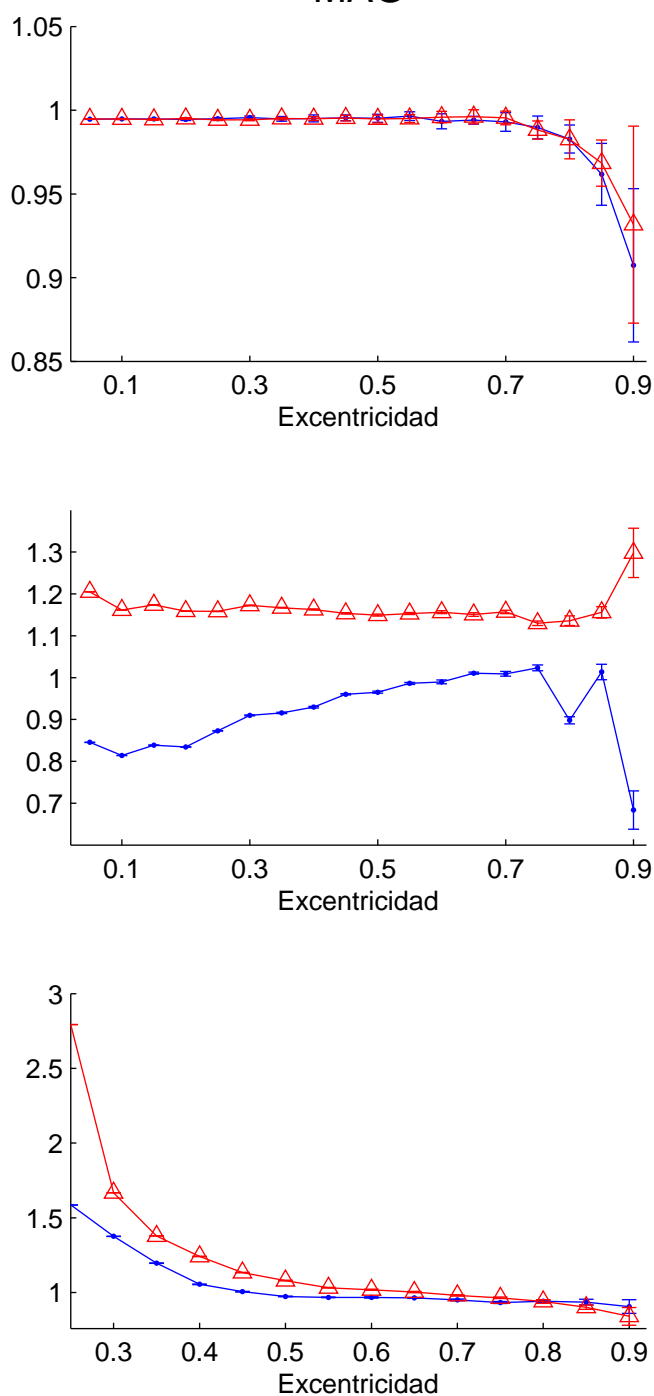

Figura 2.3: Variación de los factores NRDM y MAG en el PD de EEG en función de la excentricidad de la fuente. Se indican los parámetros considerando diferentes medios conductores (en distintas filas de la figura) y fuentes dipolares orientadas radial (azul) y tangencialmente (rojo).

\subsubsection{Resultados en MEG}

Por último se procedió a validar el método propuesto para la resolución del PD en MEG. En este caso, a diferencia de EEG, existe solución analítica para el caso esférico sólo bajo la suposición de conductividad eléctrica isótropa (Sarvas, 1987). 


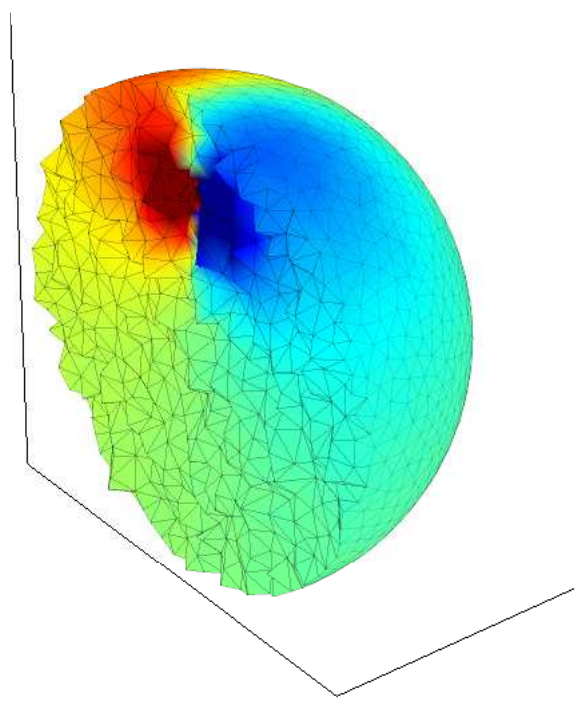

(a)

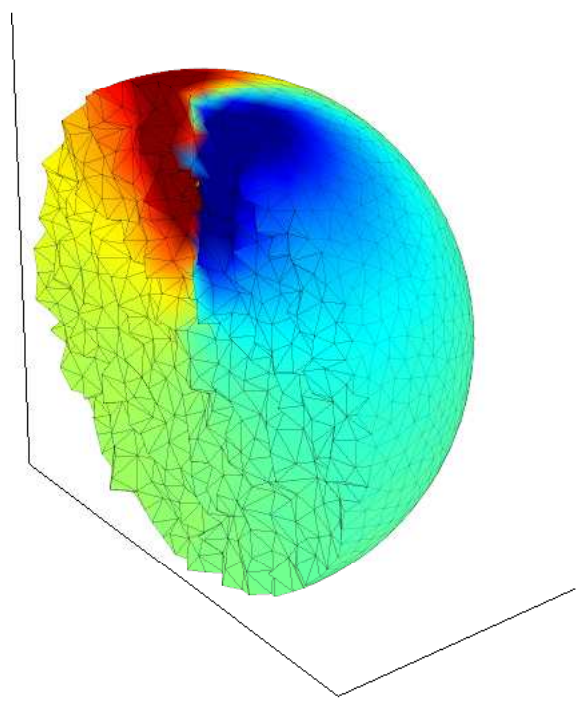

(b)

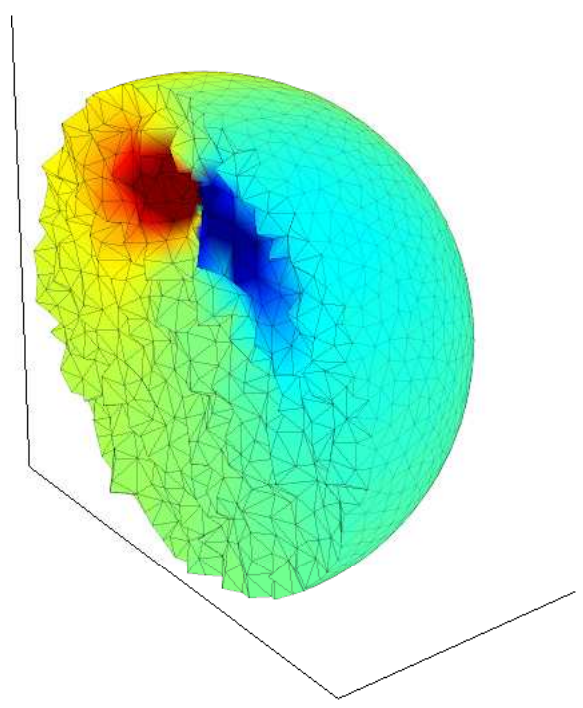

(c)

Figura 2.4: Influencia del tensor de conductividad en la determinación del potencial de la esfera considerando (a.) conductividad isótropa, (b.) conductividad anisótropa radial $\left(\sigma_{r}=10 \sigma_{t}\right)$ y (c.) conductividad anisótropa tangencial $\left(\sigma_{t}=10 \sigma_{r}\right)$. 
NRDM

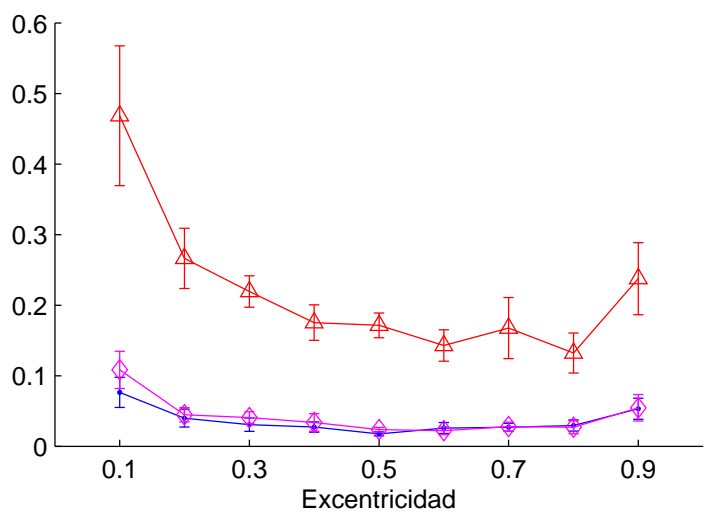

MAG

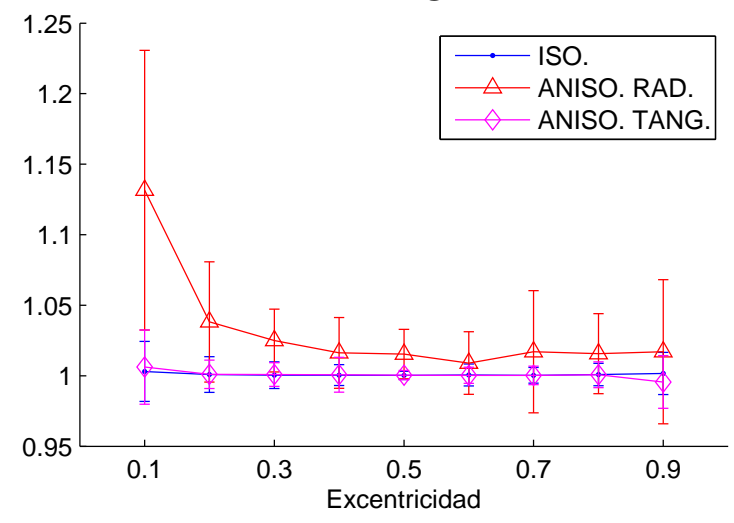

Figura 2.5: Variación de los factores NRDM y MAG en el PD de MEG en función de la excentricidad de la fuente. Se indican los parámetros de error respecto de la solución analítica para conductividad eléctrica isótropa. Los resultados corresponden a fuentes orientadas tangencialmente y medios isótropos (azul), aisótropo radial (rojo) y anisótropo tangencial (magenta).

Por ello se procedió a comparar la solución del campo magnético considerando la conductividad isótropa y anisótropa, respecto de la solución analítica. Esto permite validar el método propuesto para el caso isótropo y evaluar la variación de la solución frente a cambios de la conductividad eléctrica respecto del primer caso. En la Figura 2.5 se muestran los errores en la reconstrucción del campo considerando fuentes orientadas tangencialmente en función de su excentricidad. La discretización utilizada es la misma a la empleada en la Figura 2.5. Cabe destacar que, al resultar el campo magnético una magnitud vectorial, los errores se calcularon sobre todas las componentes, i.e. se apiló el vector de $N_{s} \times 3$ en uno de $3 N_{s} \times 1$ y se computaron los errores sobre éste último. Asimismo, se muestran solo resultados considerando fuentes orientadas tangencialmente debido a que el para fuentes orientadas radialmente y medios esféricos isótropos el campo magnético resultante es nulo (Mosher et al., 1999). Se ve claramente que, para la discretización utilizada, los errores son pequeños a pesar de la escasa cantidad de elementos. Asimismo, puede verse la notable similaridad de los resultados considerando conductividad eléctrica anisótropa tangencial e isótropa, no ocurriendo lo mismo con el caso anisótropo radial.

Con la finalidad de evaluar la influencia de la anisotropía en la determinación del 


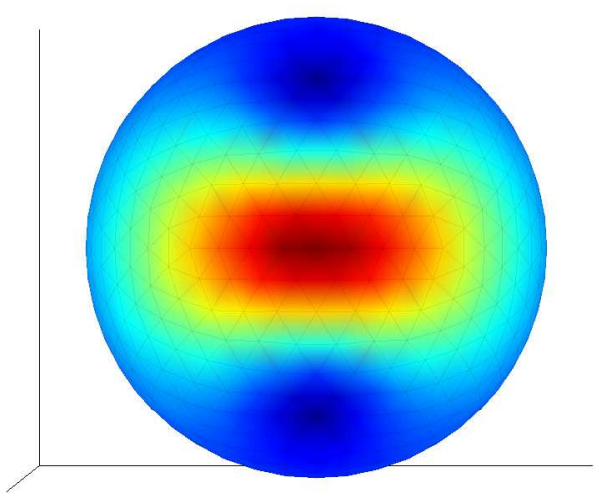

(a)

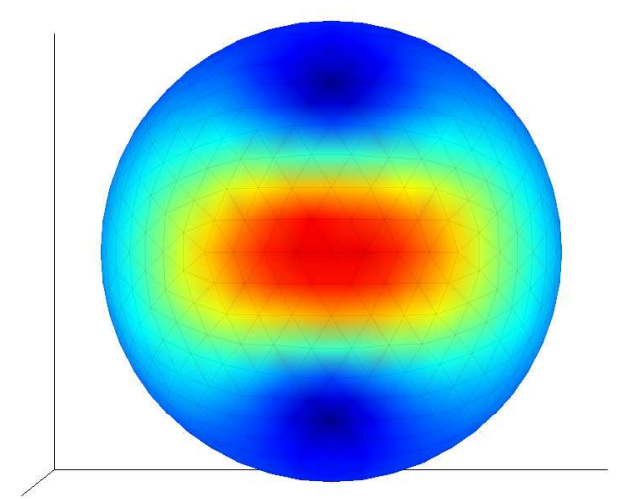

(b)

Figura 2.6: Influencia del tensor de conductividad en la determinación del campo magnético secundario sobre la esfera considerando (a.) conductividad isótropa $\left(\sigma_{r}=\sigma_{t}\right)$ y (b.) conductividad anisótropa radial $\left(\sigma_{r}=10 \sigma_{t}\right)$.

campo magnético se calculó el campo secundario para la fuente y el modelo considerado para la Figura 2.4. En la Figura 2.6 se muestran los resultados considerando conductividad isótropa (a) y anisótropa radial (b.). Se ve una leve variación de los campos secundarios, influyendo en mayor proporción a medida que la componente radial de la conductividad eléctrica aumenta. Estas diferencias se deben fundamentalmente a las variaciones de la corriente de volumen con la conductividad eléctrica. En la Figura 2.7 se muestra dicha corriente para cada uno de los casos anteriores, en donde se ve claramente como la conductividad eléctrica determina la distribución de la densidad de corriente, la cual puede determinar diferencias significativas en el campo magnético medido. 


\subsection{Conclusiones}

En este capítulo se presentó el PD en E/MEG considerando el MEF. Este método constituye una solución viable a la resolución del problema directo en E/MEG sobre medios no estándar, como lo es la cabeza humana. A diferencia de algunos de los métodos existentes, el planteado permite realizar la adecuada caracterización del medio (es decir, el encéfalo) tanto geométrica como eléctricamente, contribuyendo ambos factores a la adecuada resolución del problema (Hallez et al., 2007; Güllmar et al., 2010).

Existen numerosos trabajos que estudian la factibilidad del MEF en la aplicación al PD en E/MEG. En muchos de ellos (van Uitert et al., 2004; Wolters et al., 2006, 2007) la implementación es prácticamente omitida, por lo que resultan un tanto distantes de la aplicación inmediata. Por esta razón se formuló un método considerando funciones de base lineales a partir de la transformación de volumen, conllevando a expresiones analíticas de muy sencilla implementación. A nuestro entender este trabajo resulta ser el primero en donde se dan expresiones directas de cálculo para la resolución del problema directo en E/MEG, con su correspondiente tratamiento teórico y análisis de errores.

Una metodología similar se planteó para EEG en Zhang et al. (2004) considerando funciones de base cuadráticas, sin llegar a expresiones tan sencillas y analíticas como las aquí planteadas. Si bien las funciones de forma cuadráticas llevan a resultados más precisos, el número de incógnitas aumenta drásticamente (de 4 incógnitas por elemento para el caso lineal a 10 para el caso cuadrático), por lo que el costo computacional resulta determinante. Para paliar este hecho, deben tomarse elementos de mayor volumen a los considerados en el método lineal, implicando una menor adherencia del modelo.

Los resultados mostrados son comparables a los presentados en los estudios citados, logrando una gran simplicidad en la implementación. Más aún, considerando el caso isótropo presenta un mejor desempeño en el PD de EEG respecto de otras implementaciones en donde se utiliza un número de nodos por demás superior al aquí utilizado (Wolters et al., 2007). Comparaciones con otros métodos serán parte 
de futuros trabajos, en donde se evalúen tanto los errores cometidos por cada uno como su costo computacional. 


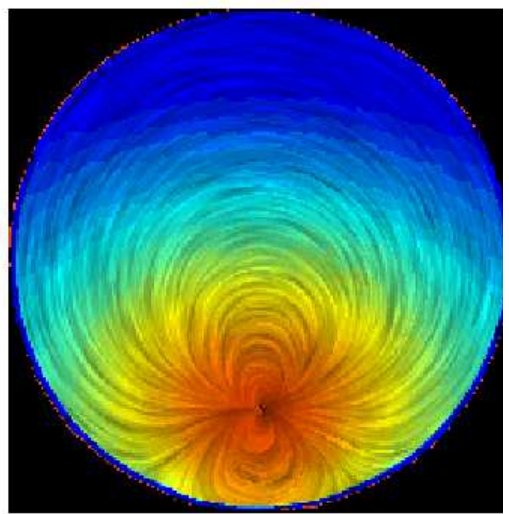

(a)

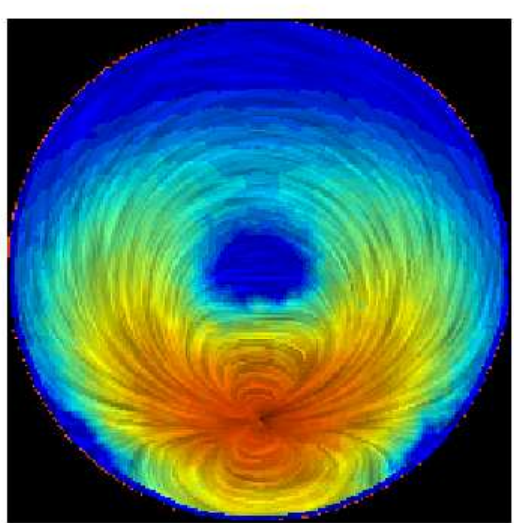

(b)

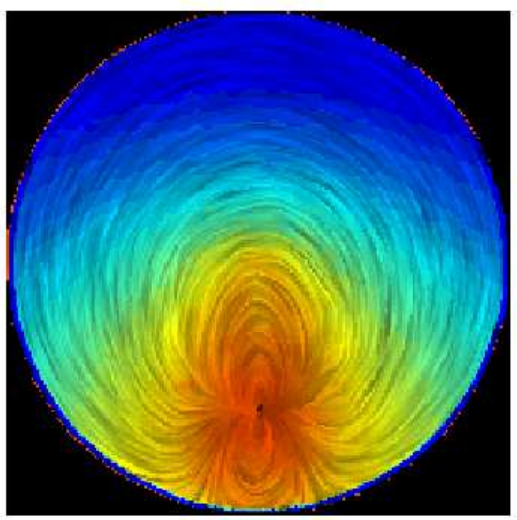

(c)

Figura 2.7: Distribución de la densidad de corriente considerando al medio conductor como isótropo (a), anisótropo tangencial (b) y anisótropo radial (c). Los colores corresponden a la magnitud de la densidad de corriente en escala logarítmica. 


\section{Capítulo 3}

\section{Influencia del modelo de cabeza en el problema inverso de EEG}

\subsection{Introducción}

Investigadores de distintos campos relacionados a las neurociencias, tales como psicólogos y médicos, emplean métodos de resolución del problema inverso (PI) con el fin de identificar a los generadores de la actividad cerebral relacionada con una cierta distribución de potencial eléctrico medido sobre el cuero cabelludo. En muchas oportunidades el fin último del estudio consiste en hallar el origen de la actividad eléctrica debida a la presentación de un estímulo determinado, estudio que lleva el nombre de potenciales evocados (PE). Éstos tienen múltiples finalidades, como la caracterización anatómico-funcional de los procesos cognitivos y la diferenciación de los mismos según se evalúen sujetos sanos o pacientes. De este modo, algunos PE resultan ser marcadores de patologías neuropsicológicas tales como bipolaridad (Sokhadze et al., 2010), prosopagnosia (Harris et al., 2005), depresión (Deldin et al., 2000) e hiperactividad (Herrmann et al., 2009), entre otros. Luego, la adecuada caracterización de las fuentes que dan lugar a dichos potenciales resulta de sumo interés debido a su carácter informativo, aportando datos significativos en la construcción de modelos de procesamiento cognitivo.

El PI asociado a PE se encuentra afectado por la incerteza del modelo utili- 
zado, tal como la geometría de la cabeza, su conductividad eléctrica y la posición de los electrodos. En relación a ello se ha mostrado que si el modelo utilizado no difiere considerablemente del modelo ideal (e individual), los efectos debidos a estas incertezas tienden a ser del mismo orden al ruido electrónico de fondo, lo que las vuelve individualmente (pero no necesariamente en forma conjunta) despreciables (von Ellenrieder et al., 2006; Güllmar et al., 2010; Beltrachini et al., 2011c). Para construir estos modelos realistas resulta necesario el conocimiento de cierta información adicional, generalmente provista por imágenes de RM y sistemas posicionadores. Sin embargo, dada la baja amplitud de los PE (no más de $10 \mu V$ ), la repetibilidad de los ensayos (a diferencia de mediciones de EEG en epilepsia) y la finalidad de caracterizar respuestas en grupos de individuos, la mayoría de los estudios de PE consisten en el análisis de múltiples realizaciones de las señales sobre una gran cantidad de personas, volviendo la adquisición de imágenes de RM y de la posición de los electrodos impráctica. Luego, un modelo genérico de cabeza debe ser utilizado.

El modelo más sencillo e históricamente aceptado es el esférico isótropo de tres capas, en donde cada una de ellas representa al cerebro, cráneo y cuero cabelludo, y las conductividades eléctricas se suponen homogéneas e isótropas (de Munck y Peters, 1993; Ermer et al., 2001). Este modelo presenta múltiples imprecisiones que afectan los problemas directo e inverso en EEG, tal como la falta de adherencia geométrica del modelo respecto a la cabeza real, así como el modelado simplificado de la conductividad eléctrica de los tejidos internos (Wolters et al., 2007). Con el objeto de evitar estos problemas se plantea el uso de modelos basados en atlas. Estos modelos se construyen mediante un promedio de cien o más sujetos sanos y generalmente conllevan a mejoras respecto de modelos sobre-simplificados (ValdésHernández et al., 2009; von Ellenrieder et al., 2009b). En el presente capítulo se consideran ambos modelos y comparan resultados en la localización de fuentes.

La influencia del modelo en los problemas directo e inverso de EEG ha sido objeto de múltiples estudios (Baillet et al., 2001; Vanrumste et al., 2002; Vatta et al., 2010). En general, éstos realizan comparaciones múltiples entre las soluciones obtenidas mediante distintos modelos, el real del individuo y aproximaciones de éste, 
considerando fuentes dipolares. Sin embargo, los modelos de cabeza a utilizar en estudios relacionados a PE son todos aproximados, por lo que un análisis comparativo con el modelo real no resulta factible. En el presente capítulo se muestra un método que permite comparar la varianza de los estimadores de densidad de corriente obtenidos con cada modelo sin contar con el conocimiento del verdadero modelo individual, basado en el popular algoritmo sLORETA (Pascual-Marqui, 2002). Mediante este método se muestra que, bajo algunas hipótesis, la varianza de los estimadores depende solamente del modelo de cabeza considerado, lo cual constituye un mecanismo de comparación válido en la reconstrucción de fuentes distribuidas.

Para realizar el análisis se utilizan datos de un estudio del PE N170. Esta componente se caracteriza por mostrar un pico negativo alrededor de los 140-200 ms posteriores al estímulo, y se ha mostrado su intervención en el procesamiento de caras y palabras (Rossion et al., 2003). La componente N170 es sensible a la clase de estímulo utilizado, así como a la valencia emocional (Itier y Taylor, 2004; Vuilleumier y Pourtois, 2007; Ibáñez et al., 2011b). El procesamiento de caras y sus emociones corresponde a uno de los pasos más importantes en el desarrollo de los comportamientos sociales cognitivos (Grossmann, 2010). Por ende, la localización precisa de las regiones corticales asociadas a su generación puede ayudar a entender este proceso tan complejo.

Múltiples estudios se han avocado a localizar los generadores de la componente N170, llegando a diferentes resultados. Algunos autores (Rossion et al., 2003; Deffke et al., 2007) han concluido en que los generadores del N170 se localizan en el Giro Fusiforme (FG), o en el FG y estructuras adicionales, tal como el Giro Lingual (LG) (Mnatsakanian y Tarkka, 2004). Otros trabajos han encontrado a los generadores de dicha componente en la corteza Temporal-Occipital (TOC) (Schweinberger et al., 2002) o en las cisuras del lóbulo temporal superior (Itier y Taylor, 2004). Estas diferencias pueden deberse a distintos factores, como ser el estímulo utilizado para su generación o el modelo de cabeza y fuente adoptado a tal fin. Como se muestra en este capítulo, la utilización de un modelo de cabeza y fuente poco realista conlleva a un aumento en la varianza de localización, por lo que su uso debe ser evitado. 
En este capítulo se distinguen entre sujetos sanos de control y pacientes con Trastorno por Déficit de Atención con Hiperactividad (TDAH). Este desorden corresponde a una condición neuropsiquiátrica que se origina en la infancia y se extiende a la vida adulta, afectando el adecuado funcionamiento neurológico. Se ha mostrado que la componente N170 representa un marcador factible en el análisis de caras y palabras en TDAH (Marsh y Williams, 2006; Ibáñez et al., 2011a). Luego, la adecuada reconstrucción de las fuentes de este PE es un factor fundamental a la hora de reportar diferencias entre sujetos de control y pacientes. Esta localización se ve sumamente afectada por la disminución del pico de amplitud de la componente medida respecto de los sujetos sanos, por lo que la influencia del modelo puede cobrar un papel preponderante en el análisis. En las siguientes secciones se analizan estas diferencias y se muestra que un modelo esférico puede conllevar a resultados erróneos, aún peores si el modelo de una única fuente dipolar es considerado.

El presente capítulo se organiza del siguiente modo: en la Sección 3.2 se describe el problema asociado a la localización de las fuentes de actividad cerebral asociadas al PE N170. Luego, se presentan los modelos considerados en el análisis y la metodología propuesta para la comparación del desempeño de éstos en la resolución del PI. En la Sección 3.3 se presentan resultados que permiten validar las hipótesis acerca de la conveniencia del modelo realista basado en atlas. Finalmente, en la Sección 3.4 se presenta una discusión sobre la importancia de los resultados obtenidos en relación a los trabajos existentes en la materia, conclusiones finales y líneas de trabajo futuro.

\subsection{Materiales y Métodos}

Este estudio es parte de un proyecto de investigación más amplio relacionado a la asociación entre el procesamiento cortical de expresiones emocionales de las caras, habilidades de cognición social y funcionamiento ejecutivo (Ibáñez et al., 2011a,b). Solo los datos relevantes se presentan en este capítulo. 


\subsubsection{Participantes}

Diez pacientes adultos con TDAH (uno masculino, tres zurdos, edad 33,1 $\pm 3,42$ años) y diez participantes sanos (uno masculino, dos zurdos, edad 33,3 $\pm 3,64$ años) se consideraron para el estudio. Los pacientes con TDAH se diagnosticaron de acuerdo al criterio de DSM-IV (American Psychiatric Association, 2000), realizado por tres expertos. Todos los pacientes se encontraban bajo tratamiento con medicación (metilfenidato), la cual fue suspendida en el día de mediciones. El diagnóstico de TDAH basado en DSM-IV se complementó con pruebas de impulsividad/hiperactividad, manía, depresión y estudios neuropsicológicos correspondientes. Todas las pruebas se realizaron con el consentimiento de los participantes, de acuerdo a la declaración de Helsinki. Más detalles acerca de la selección de pacientes puede encontrarse en Ibáñez et al. (2011b).

\subsubsection{Estímulos de potenciales evocados}

Con el fin de obtener los potenciales evocados correspondientes a la componente N170 se presentaron imágenes de caras $(\mathrm{C})$, palabras $(\mathrm{P})$ y caras y palabras en simultáneo $(\mathrm{C}+\mathrm{P})$ a los participantes. Se pidió a los sujetos categorizar P y C dispuestas en un monitor tan rápido como sea posible. En la Figura 3.1 se muestra un diagrama del experimento realizado. Cada realización se iniciaba con una cruz de fijación por $1000 \mathrm{~ms}$. Luego, se presentaba un estímulo durante $100 \mathrm{~ms}$, seguido de una cruz de fijación hasta que los participantes respondieran. Si la respuesta resultaba ser incorrecta, se mostraba una cruz de fijación roja como realimentación por 100 ms y la realización se daba por concluida. No se daba realimentación en caso de una respuesta acertada. Luego de cada realización, se agregaba un período inter-estímulos de $1000 \mathrm{~ms}$.

Respecto a la prueba de bloques simultáneos, a cada participante se le mostraba una cara en el centro y una palabra por debajo, dispuestas simultáneamente durante 100 ms. A ellos se les pedía indicar la emoción mostrada por la cara ignorando la palabra. En los estudios de $\mathrm{P}$ y $\mathrm{C}$ individualmente, a los participantes se les pidió categorizar la emoción de la cara o palabra correspondiente como positiva o 
FIJACIÓN

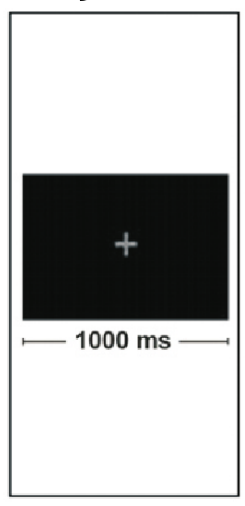

ESTÍMULO

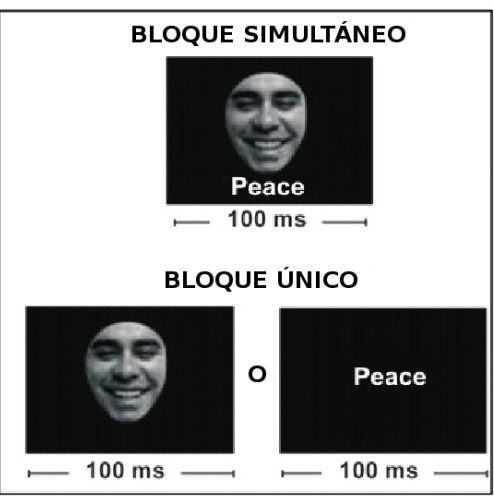

FIJACIÓN

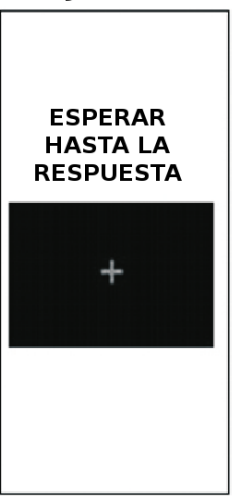

RESPUESTA REALIM.

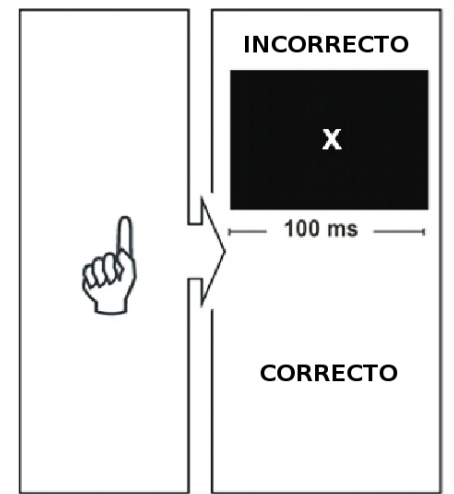

Figura 3.1: Diseño experimental de la generación del PE. Ver la Sección 3.2.2 para su explicación en detalle.

negativa. Más detalles acerca de las condiciones del experimento pueden encontrarse en Ibáñez et al. (2011b).

\subsubsection{Adquisición de datos}

Las señales de EEG se adquirieron con un sistema de 128 canales de BioSemi (BioSemi, Amsterdam, Países Bajos). La frecuencia de muestreo se fijó en $500 \mathrm{~Hz}$ y las señales se filtraron entre $0,3 \mathrm{~Hz}$ y $30 \mathrm{~Hz}$. Luego, los datos fueron sub-muestreados a $128 \mathrm{~Hz}$ y re-referenciados al potencial eléctrico promedio instantáneo. Con la finalidad de remover la contaminación debida al movimiento ocular se utilizaron dos derivaciones de supervisión vertical y horizontal, que permitieron su remoción mediante inspección visual y algoritmos automáticos (Gratton et al., 1983). Sólo segmentos libres de artefactos fueron promediados para la obtención de los PE.

\subsubsection{Modelo de cabeza y problema directo}

Con la finalidad de resolver el PI debe escogerse un modelo de cabeza. Dado que las RM de los sujetos y pacientes no se encontraban disponibles, dos modelos estándar fueron considerados. El primero de ellos es el ampliamente utilizado modelo esférico isótropo de tres capas, en donde cada capa representa a los diferentes tejidos 
de la cabeza (i.e. cerebro, cráneo y cuero cabelludo). Las conductividades eléctricas adoptadas para cada capa son los aceptadas $0,33 \mathrm{~S} / \mathrm{m}$ para el cerebro y el cuero cabelludo, y 0,0096 S/m para el cráneo (Dannhauer et al., 2010).

El segundo modelo adoptado en este análisis corresponde a un modelo promedio construido a partir de un total de 152 RM de participantes sanos provisto por el Consorcio Internacional de Mapeado del Cerebro (ICBM) (Mazziotta et al., 2001). Las superficies del cuero cabelludo y el cráneo se extrajeron mediante el uso de la utilidad de extracción del cerebro BET (Jenkinson et al., 2005) y luego corregidas utilizando MeshLab ${ }^{1}$. Con el fin de realizar un modelo lo más realista posible se utilizó el mapa de anisotropía cerebral promedio basado en imágenes de RM por tensor de difusión tomadas a 81 participantes sanos (Mori et al., 2008) co-registrados con el modelo ICBM ${ }^{2}$. Esto permite considerar a la conductividad eléctrica del cerebro como un campo tensorial a partir de una transformación lineal en los autovalores de dichos tensores (Tuch et al., 2001). La conductividad eléctrica del cuero cabelludo y el hueso se considera de igual forma que en el modelo esférico. Dado que este modelo captura en forma realista tanto la geometría como la conductividad eléctrica, en lo siguiente se lo llamará indistintamente modelo realista o promedio.

En la Figura 3.2(a) se muestra un corte sagital en donde las superficies entre capas del modelo esférico (líneas delgadas) y el modelo realista (líneas gruesas) se superponen. Las diferencias geométricas entre ellos se vislumbran con facilidad. En la Figura 3.2(b) se muestra, para el mismo corte, el mapa de conductividad eléctrica (conductividad media en cada elemento del mallado) considerada para el modelo realista. La adecuada consideración de las variaciones de la conductividad en el cerebro son de importancia (Wolters et al., 2006; Güllmar et al., 2010), especialmente debido a la presencia del LCR, el cual ha mostrado ser de suma influencia en las soluciones de los problemas directo e inverso considerando modelos individuales (Ramon et al., 2006; Wolters et al., 2007; Wendel et al., 2008).

Con el objeto de resolver el problema directo en EEG se utilizó el MEF desarrollado en el Capítulo 2. Los modelos originales se discretizaron en aproximadamente

\footnotetext{
${ }^{1}$ Disponible en http://meshlab. sourceforge.net

${ }^{2}$ Disponible en http://www.loni.ucla.edu/ICBM/Downloads/Downloads_DTI-81.shtml
} 


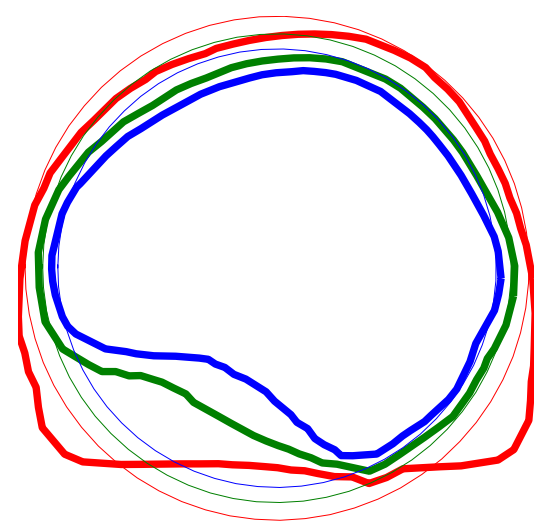

(a)

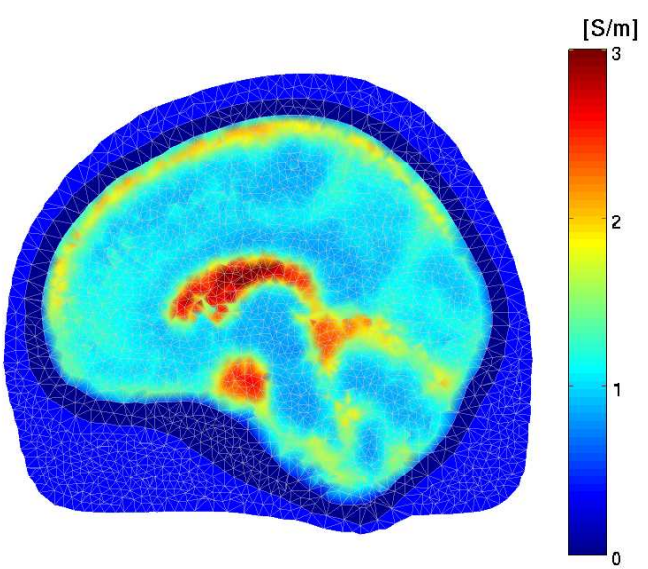

(b)

Figura 3.2: Modelos de cabeza utilizados en el presente análisis. (a) Superposición de los modelos realista (líneas gruesas) y esférico (líneas finas) sobre un corte sagital. (b) Disctretización del modelo realista coloreado de acuerdo al mapa de conductividad eléctrica (conductividad media en cada elemento).

450.000 tetraedros utilizando el paquete ISO2Mesh ${ }^{3}$ (Fang y Boas, 2009), resultando en una malla con elementos de $2 \mathrm{~mm}$ de longitud de lado promedio (Figura 3.2(b)). Se restringieron las ubicaciones de las fuentes de actividad cerebral a la superficie cortical (discretizada en 8000 nodos) con orientación libre, tal como se sugiere en Valdés-Hernández et al. (2009).

Debe notarse que, con el fin de confinar a la superficie cortical en el interior del cráneo, como se muestra en la Figura 3.2(a), el modelo esférico se ajustó al modelo promedio del mejor modo posible.

\subsubsection{Localización de fuentes}

Con objeto de localizar a los generadores de la actividad cerebral bajo estudio se consideró el algoritmo sLORETA (Pascual-Marqui, 2002). Éste es un método estadístico de localización de fuentes distribuidas espacialmente de forma suave que no presupone el número de focos de actividad cerebral. Si bien su resultado es un mapa de activación distribuido, se ha mostrado que sLORETA permite localizar

\footnotetext{
${ }^{3}$ Disponible en http://iso2mesh.sourceforge.net/cgi-bin/index.cgi
} 
fuentes dipolares en forma exacta en la ausencia de ruido (Greenblatt et al., 2005). A pesar de que esta condición nunca se cumple en casos reales, el método sLORETA ha resultado ser sumamente eficiente en la localización de fuentes dipolares (Sekihara et al., 2005) y distribuidas (Olbrich et al., 2009), volviéndolo un método apropiado y ampliamente considerado en la literatura relacionada a PE (Weber et al., 2005; Sumiyoshi et al., 2009; Utama et al., 2009).

Básicamente, sLORETA estima la densidad de corriente con restricción de norma mínima, para luego obtener la localización de la fuente mediante la estandarización de los valores encontrados con respecto a la varianza de dichos estimadores. Esto conlleva a resultados en forma de potencia de densidad de corriente estandarizada (SCDP), la cual denota activación cortical. Cuanto mayores sean los valores de SCDP, mayor será la evidencia de activación cortical. La restricción de mínima norma impuesta por el método conlleva a una solución suave, cuya interpretación fisiológica resulta en la activación sincrónica de poblaciones neuronales vecinas, disponiendo sólo de cambios graduales en la orientación. Esta suposición es apoyada por estudios propios de la electrofisiología, en donde se ha mostrado que las poblaciones neuronales contiguas muestran una actividad altamente correlacionada (Vaadia et al., 1995; Haalman y Vaadia, 1997). Para más detalles del método se remite al Apéndice B.1 o a la publicación original (Pascual-Marqui, 2002).

El método de sLORETA permite también estudiar el error introducido en caso de considerar el modelo de fuente dipolar equivalente, ampliamente utilizado en la literatura relacionada a la localización de las fuentes que originan la componente N170 (Shibata et al., 2002; Schweinberger et al., 2002; Watanabe et al., 2003; Rossion et al., 2003; Mnatsakanian y Tarkka, 2004; Deffke et al., 2007). Éste es un modelo muy sencillo, pero su simplicidad puede conllevar a resultados erróneos en caso de que las hipótesis para la validez del modelo no sean cumplidas, i.e. el área de corteza involucrada en la generación del PE no es lo suficientemente pequeña (Kobayashi et al., 2005). En este capítulo se considera al punto con mayor SCDP como la mejor solución de fuente dipolar equivalente, debido a la ausencia de error en condiciones ideales. 


\subsubsection{Comparación de los modelos de cabeza}

Cuando no existen suposiciones a-priori acerca de la ubicación e interacción de las fuentes, puede mostrarse que la varianza del estimador de la densidad de corriente resultante del método sLORETA sólo depende del modelo de cabeza utilizado (ver Apéndice B.1). Luego, esta varianza puede actuar como indicadora de las diferencias de resultados obtenidos mediante los diferentes modelos de cabeza, permitiendo comparar las influencias de los mismos en la solución del PI.

Con el fin de cuantificar el efecto del modelo en la solución del PI de EEG se consideró calcular el cociente entre las varianzas totales de los estimadores de la densidad de corriente correspondientes a los modelos esférico y realista, aquí llamada relación de varianza $\gamma$. Matemáticamente

$$
\gamma_{l}=\frac{\operatorname{tr}\left\{\left[\mathbf{S}_{\mathbf{j}}^{e}\right]_{l}\right\}}{\operatorname{tr}\left\{\left[\mathbf{S}_{\mathbf{j}}^{r}\right]_{l}\right\}},
$$

en donde $\left[\mathbf{S}_{\mathbf{j}}^{e}\right]_{l}$ y $\left[\mathbf{S}_{\mathbf{j}}^{r}\right]_{l}$ son las matrices de covarianza de dimensión $3 \times 3$ correspondientes a los estimadores de la densidad de corriente $\mathbf{j}$ asociada a los modelos esférico y realista, respectivamente, en la l-ésima posición sobre la corteza. Luego, el parámetro $\gamma$ permite comparar la varianza de los estimadores de la densidad de corriente considerando ambos modelos. En el Apéndice B.1 se muestra que, en caso de que la densidad de corriente estimada es del mismo orden para los modelos esférico y realista, la relación de varianza tendrá en cuenta a las diferencias en el factor SCDP, el cual será responsable por las diferencias en la localización de las fuentes. Valores de $\gamma>1$ se encontrarán asociados a regiones corticales en donde el modelo promedio resulta mejor (menor varianza en la reconstrucción de la fuente) que el modelo esférico.

\subsubsection{Selección de datos}

Con la finalidad de obtener las imágenes de SCDP correspondientes a la componente N170 se consideraron los picos de las componentes negativas del PE. Como ejemplo de ello, en la Figura 3.3 se muestran los valores promedio de los potenciales 


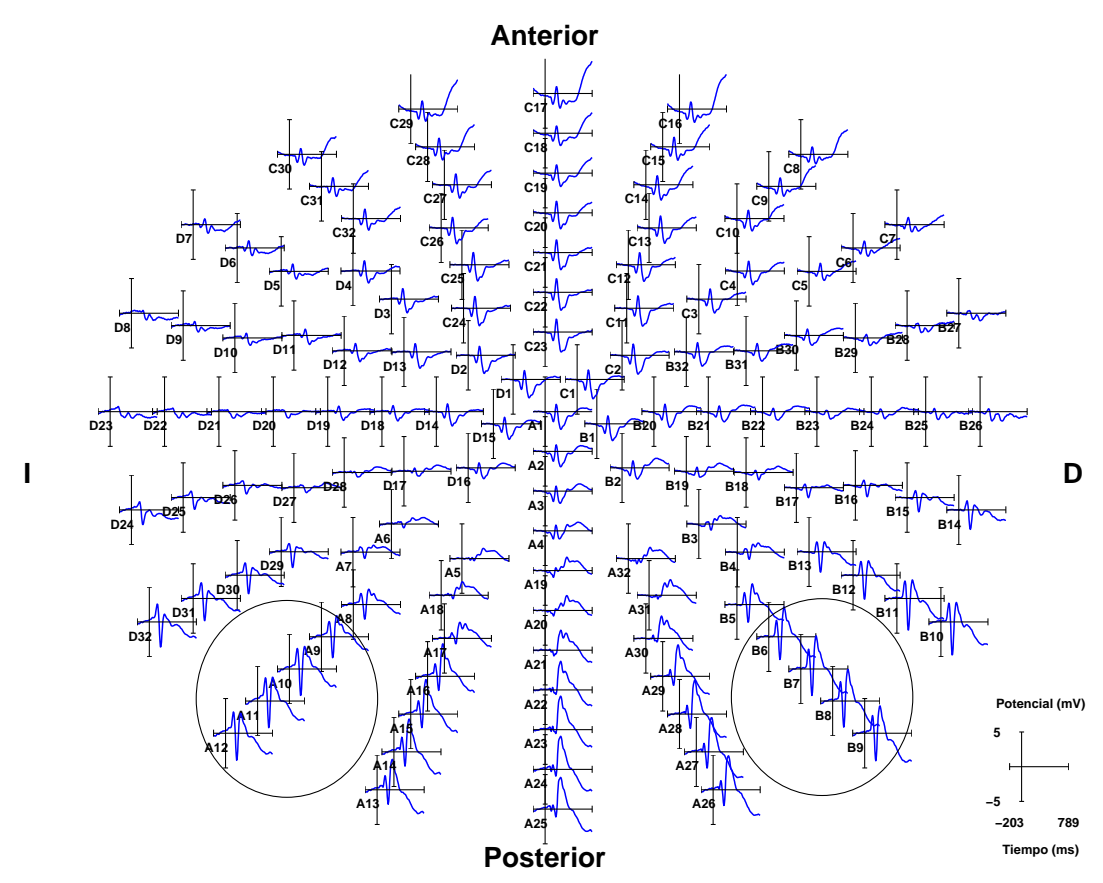

Figura 3.3: Promedio de los potenciales eléctricos medidos en la configuración estándar de 128 electrodos de BioSemi, considerando C como estímulos. Los electrodos representativos se encuentran marcados (ver Sección 3.2.7).

eléctricos medidos sobre sujetos control considerando estímulos de C, ubicados en la posición de 128 electrodos estándar de BioSemi ${ }^{4}$.

Puede verse que el potencial eléctrico correspondiente a la componente bajo estudio presenta una distribución bilateral, tal como se reportó en algunos estudios (Rossion y Jacques, 2008), extendiéndose alrededor de los electrodos A9-A12 y B6-B9 (i.e. P8/P7 y PO8/PO7 en el sistema estándar). Para determinar el pico del $\mathrm{PE}$ se consideró la señal promedio en dichos electrodos representativos, considerando una ventana temporal de 167-229 ms para C y C+P, y de 182-284 ms para $\mathrm{P}$, para cada sujeto. Luego, el pico de amplitud de la componente N170 se consideró como el mínimo de dicho promedio.

En la Figura 3.4 se muestra el promedio de la señal medida en los canales TO representativos considerando $\mathrm{C}(\mathrm{a}), \mathrm{C}+\mathrm{P}(\mathrm{b})$ y $\mathrm{P}$ (c) como estímulos. Con diferentes

\footnotetext{
${ }^{4}$ Disponible en http://www.biosemi.com/
} 


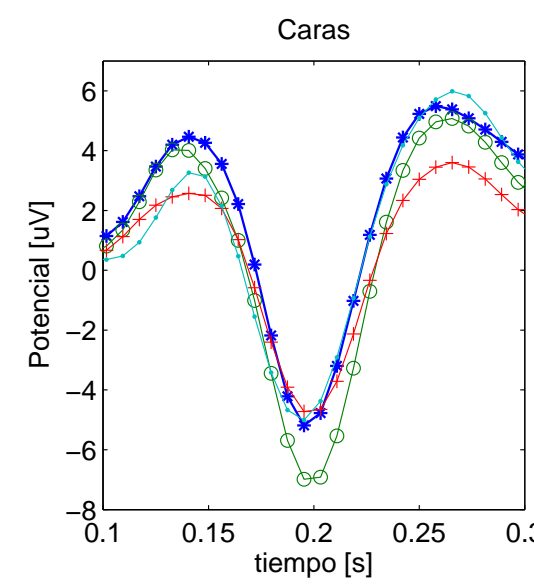

a.

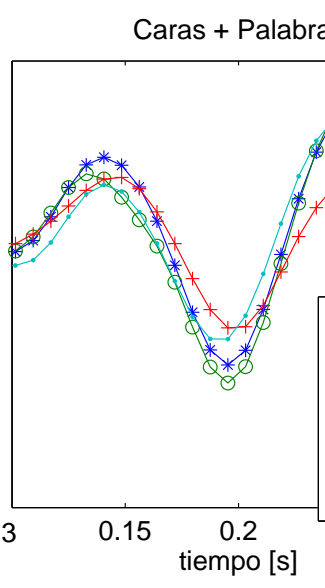

b.

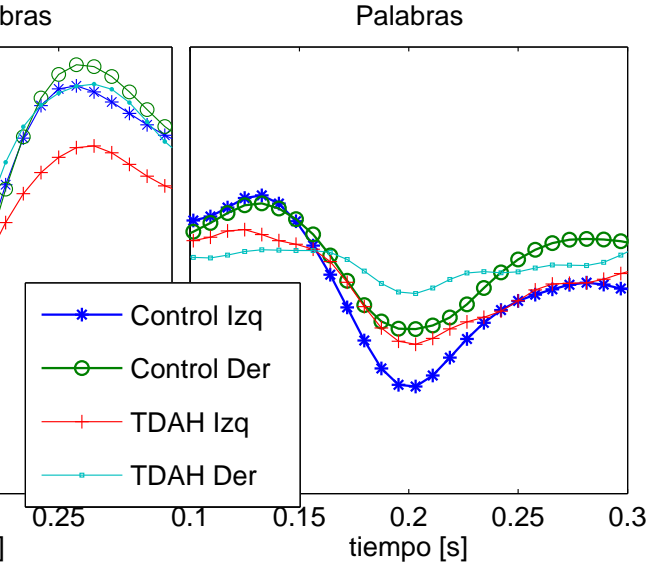

Figura 3.4: Detalle del PE N170. Se muestra el promedio del potencial eléctrico medido en los electrodos representativos (en $\mu V$ ) considerando $\mathrm{C}(\mathrm{a}), \mathrm{C}+\mathrm{P}(\mathrm{b}), \mathrm{y} \mathrm{P}$ (c) como estímulos. Con diferentes marcadores se muestran la ubicación de los electrodos (derecha o izquierda) y la naturaleza del individuo (control o paciente).

marcadores se distingue entre sujetos de control y pacientes con TDAH, así como entre mediciones con electrodos posicionados en el hemisferio derecho como izquierdo.

Puede observarse que la amplitud del promedio de los PE en los electrodos representativos es mayor en los electrodos posicionados en el hemisferio derecho cuando se consideraron tanto los estímulos de $\mathrm{C}$ como los de $\mathrm{C}+\mathrm{P}$, mientras que es mayor en los ubicados en el hemisferio izquierdo cuando en el caso de presentar sólo P. Este resultado cualitativo permite suponer que las fuentes debidas a los dos primeros estímulos se encuentran ubicadas en la TOC derecha, mientras que la TOC izquierda parece generar a las fuentes debidas a estímulos del tipo de palabras. Puede verse también que las señales medidas en pacientes con TDAH se encuentran más atenuadas y menos lateralizadas que las correspondientes a sujetos de control, como se destaca en Sprengelmeyer y Jentzsch (2006). 


\subsection{Resultados}

En esta sección se muestran resultados correspondientes a la localización de la componente N170 y el estudio de la influencia del modelo de cabeza utilizado, considerando sujetos control y pacientes con TDAH. En principio se muestra la influencia de los modelos en la resolución del PI y su comparación mediante el análisis de la relación de varianza. Luego, se ejemplifican estas diferencias en la resolución del PI utilizando fuentes distribuidas y puntuales, remarcando los errores a los que conllevan las simplificaciones sobre el modelo de cabeza y fuente.

\subsubsection{Comparación de modelos de cabeza}

Inicialmente se procedió a calcular la relación de varianza introducida en la Sección 3.2.6. Ésta permite comparar en forma directa los efectos de cada modelo en la reconstrucción de fuentes. Para ello resulta primordial la verificación de la hipótesis planteada, i.e. las densidades de corriente reconstruidas utilizando ambos modelos son del mismo orden. En la Figura 3.5 se muestra, desde un punto de vista basal, el cociente entre las densidades de corrientes estimadas utilizando el modelo realista y el esférico, para cada grupo y estímulo presentado. Se ve que la densidad de corriente estimada utilizando los dos modelos es del mismo orden en todos los casos, sobre todo en la TOC, en donde se supone que las fuentes asociadas al PE N170 se encuentran. Luego, el cálculo de la relación de varianza y su utilización para la comparación de la influencia de los modelos en la resolución del PI resulta factible.

En la Figura 3.6 se muestra la relación de varianza entre los modelos esférico y realista, desde un punto de vista superior (izquierda) y basal (derecha). Puede verse que $\gamma$ es predominantemente mayor a la unidad, lo cual significa que el modelo realista de cabeza es generalmente de mayor confianza al modelo esférico. Debe notarse que la relación de varianza aumenta en regiones en donde el modelo esférico difiere (geométricamente) del modelo realista, tal como en zonas superior de los lóbulos frontal y parietal (corteza motora superior), así como en regiones frontales, occipitales y temporales. En dichas regiones, de sumo interés para la localización del 

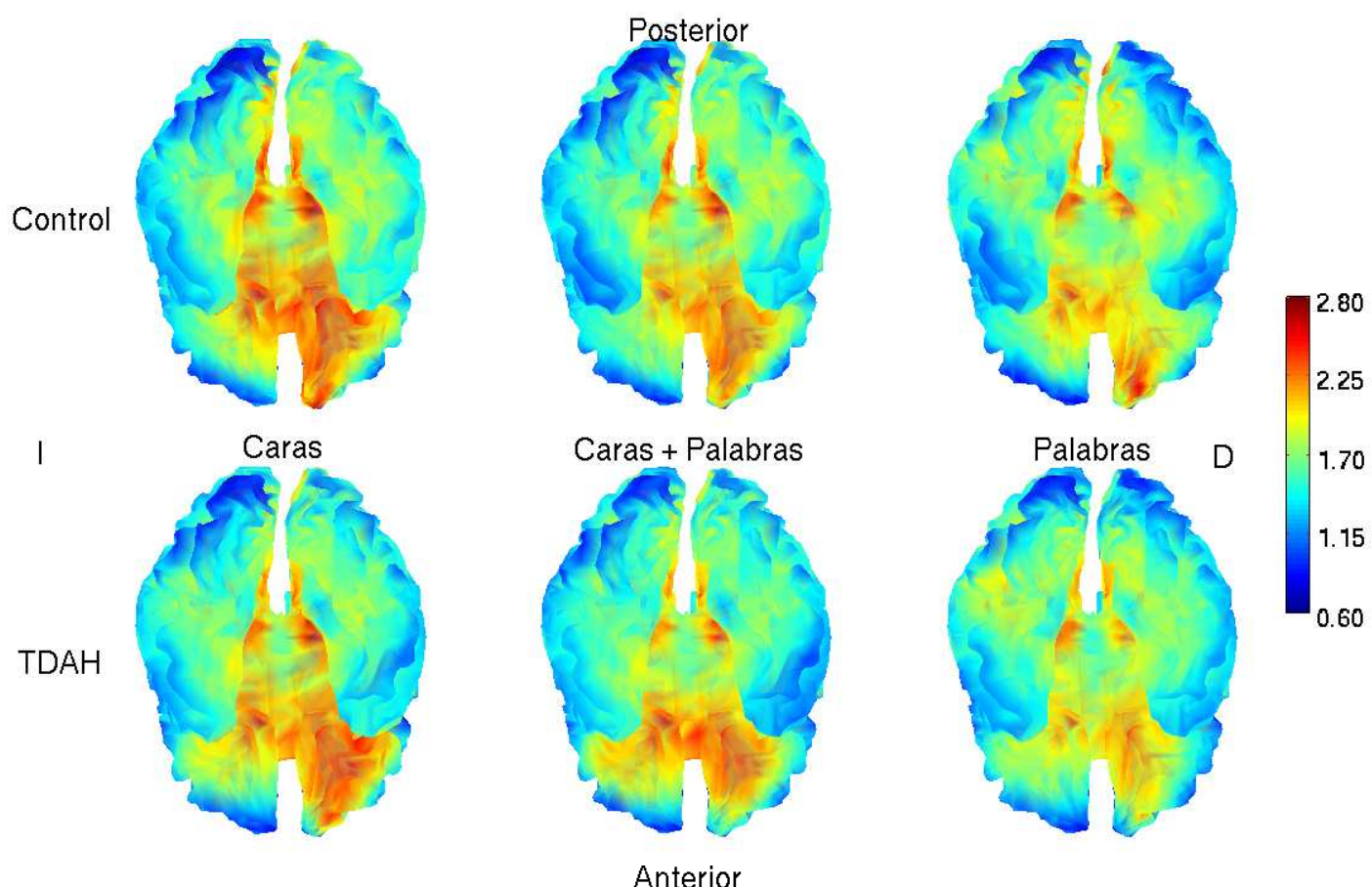

Figura 3.5: Cociente entre las densidades de corriente estimadas considerando el modelo de cabeza realista y esférico. Los resultados se muestran para sujetos control (arriba) y pacientes con TDAH, considerando C (izquierda), $\mathrm{C}+\mathrm{P}$ (centro) y $\mathrm{P}$ (derecha) como estímulos.

PE N170, la relación de varianza llega a tomar valores de hasta 5 veces.

En la Figura 3.7 se muestra un histograma que denota el porcentaje de corteza cerebral correspondiente a cada valor de $\gamma$. Puede verse que la varianza introducida por el modelo esférico es generalmente mayor a la introducida por el modelo realista, cumpliendo esta relación más del $81 \%$ de la superficie cortical.

\subsubsection{Localización de fuentes}

Una vez caracterizadas las regiones con mayor varianza debida al modelo de cabeza utilizado se procedió a localizar las fuentes de actividad cerebral de acuerdo a lo dispuesto en la Sección 3.2.5. Se calculó el SCDP para cada condición y grupo de individuos utilizando sLORETA en el pico negativo del PE N170, considerando los modelos realista y esférico. En las Figuras 3.3.2 y 3.3.2 se muestran los promedios 


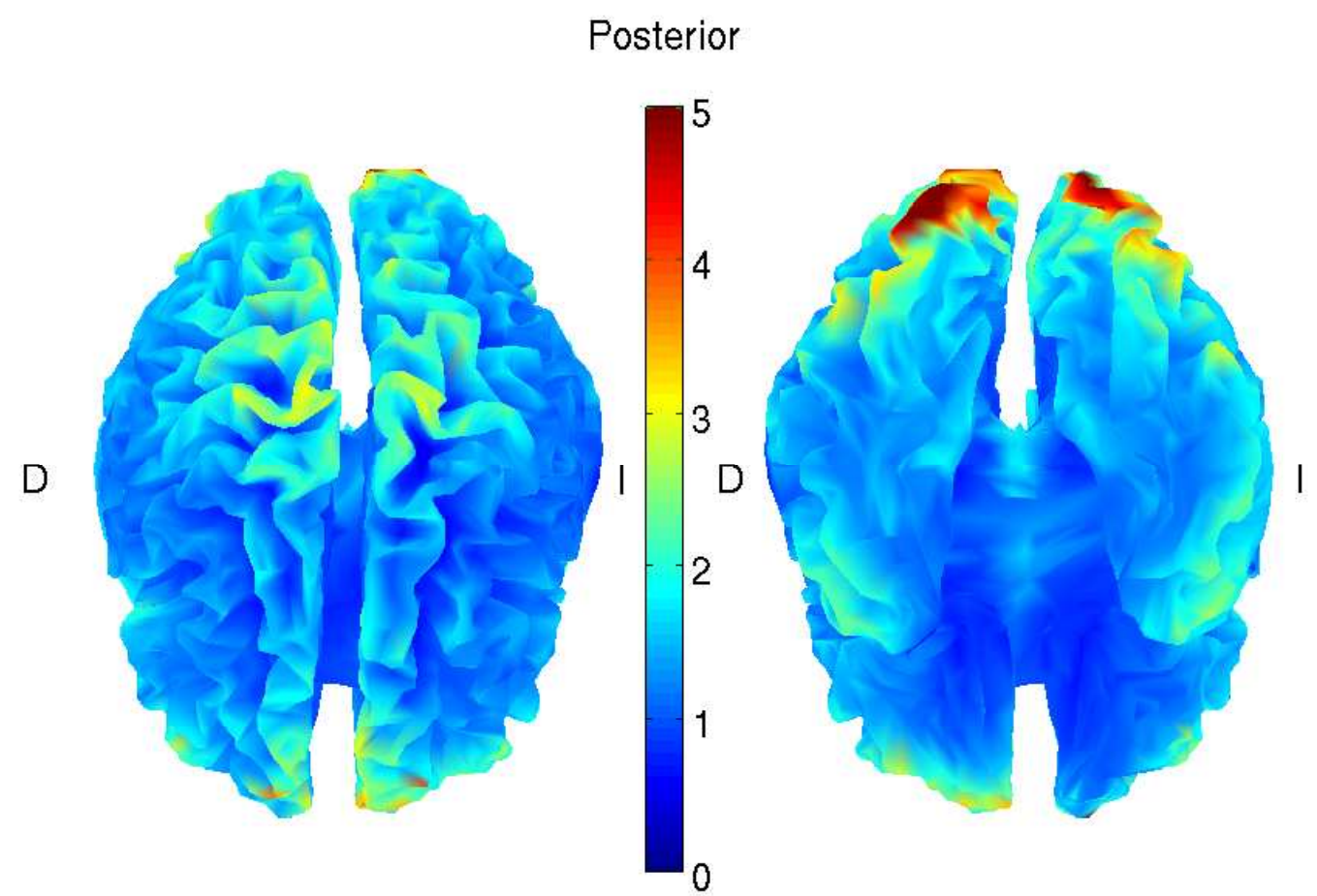

Anterior

Figura 3.6: Vista superior (izquierda) y basal (derecha) de la superficie cortical mostrando a la relación de varianza. Valores mayores se encuentran asociados a regiones corticales en donde el desempeño del modelo realista supera al del modelo esférico. Nótese que el el OP y en el OFG la relación llega a ser de aproximadamente 5.

(en las realizaciones) del SCDP para los sujetos control y pacientes con TDAH, respectivamente. Se diferencian los resultados de acuerdo al estímulo presentado (C, $\mathrm{C}+\mathrm{P}$ y P) y al modelo de cabeza utilizado (realista y esférico).

Puede observarse que en caso de utilizar el modelo esférico la región de activación se encuentra en zonas más anteriores y menos lateralizadas que en caso de considerar el modelo realista, conllevando a diferentes regiones de activación cortical. Esto concuerda plenamente con los resultados de la relación de varianza, en donde se encontró que el modelo esférico introduce mayor varianza en la localización que el modelo realista, especialmente en la TOC, lo que permite afirmar que la uti- 


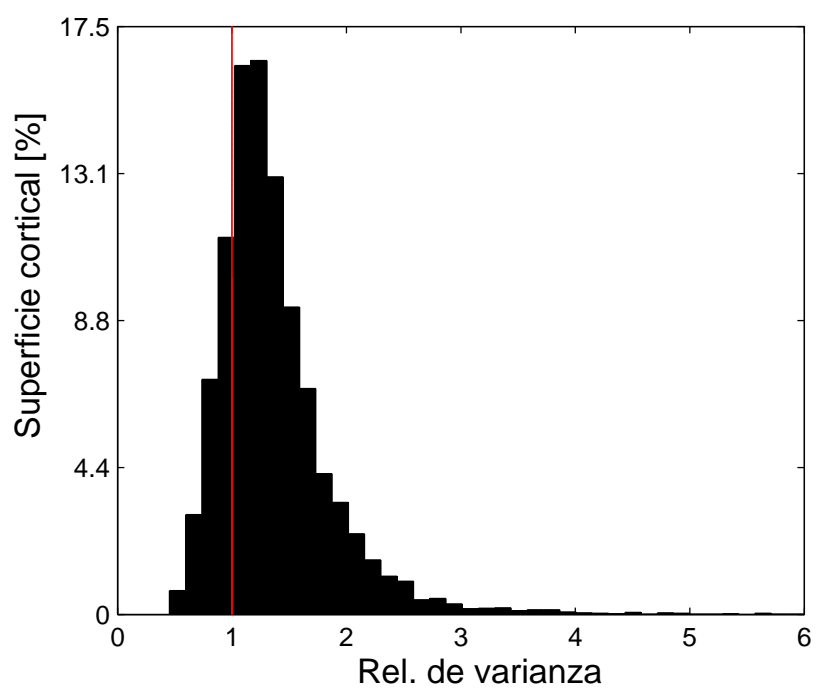

Figura 3.7: Porcentaje de área cortical asociada a distintos valores de la relación de varianza. El umbral marcado en rojo indica el límite para el cual un modelo es mejor al otro. Se consideró un tamaño de compartimiento de 0,14 .

lización de un modelo poco realista influye notoriamente en los resultados. Además, la intensidad del SCDP resultó ser notoriamente mayor cuando el modelo realista fue considerado, implicando soluciones más fidedignas del PI. Estas diferencias se mantienen para ambos grupos y estímulos.

Las diferencias marcadas resultan aún mayores en el caso de considerar el modelo de una única fuente dipolar equivalente, posicionada en el punto con mayor valor de SCDP. En el Cuadro 3.1 se muestran las ubicaciones de dichas fuentes dipolares estimadas considerando los distintos grupos de individuos y estímulos. Dado que resulta de interés determinar la región de la corteza en la cual la fuente de actividad cerebral se origina se consideró el uso del atlas de Harvard-Oxford (Desikan et al., 2006), incluido en el programa FSL. Éste es un atlas probabilístico que cubre 48 regiones de la corteza obtenido a partir de 37 RM de sujetos sanos registradas en forma afín con el modelo de ICBM utilizado. Haciendo uso de dicho atlas se buscaron las zonas en los que las fuentes dipolares se encontraban, las cuales se detallan en el mismo cuadro.

Puede verse que, en el caso de considerar el modelo realista, los valores máximos 

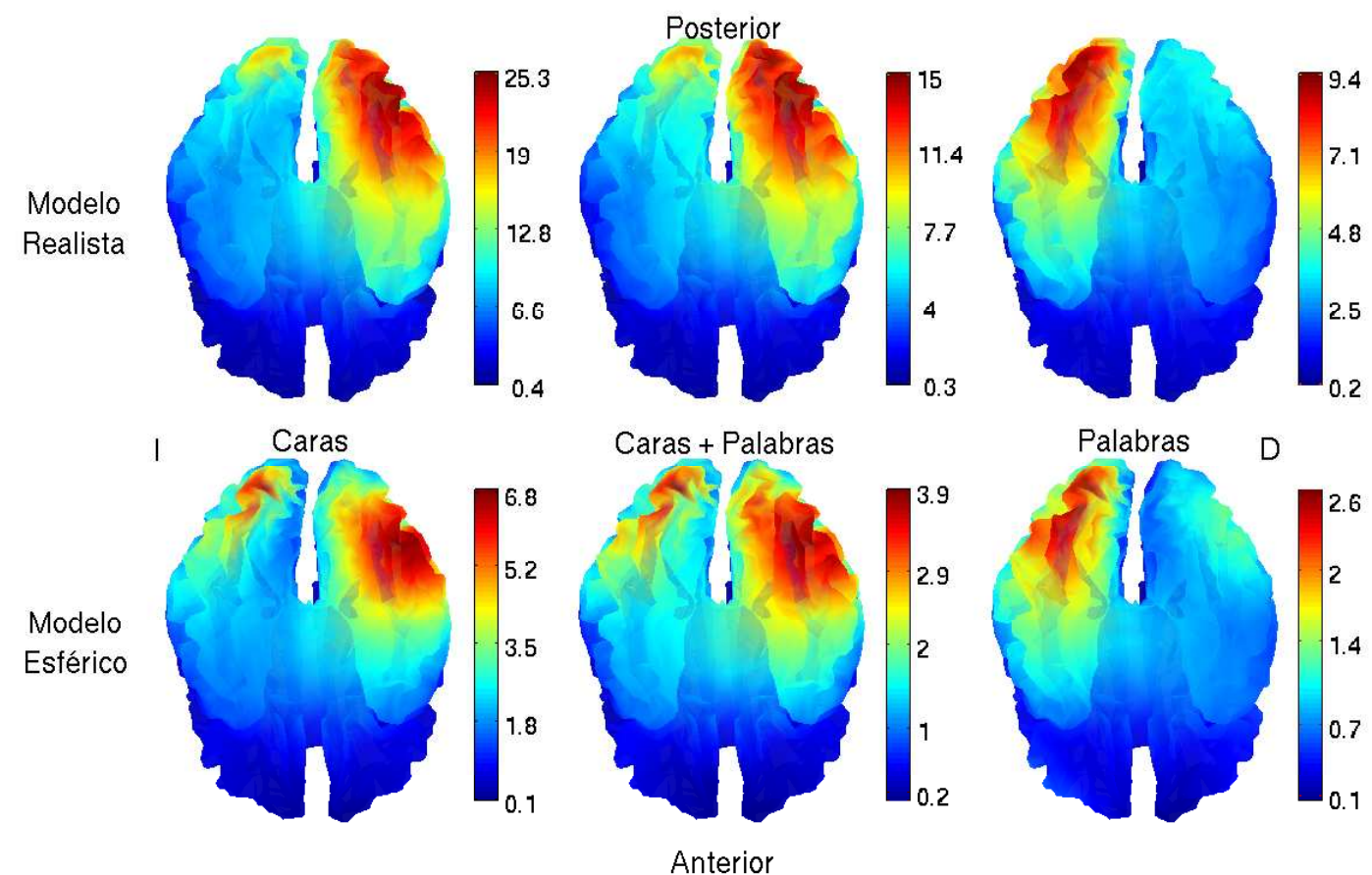

Figura 3.8: $\operatorname{SCDP}\left(\times 10^{-6}\right)$ para sujetos control. Se muestran resultados considerando el modelo de cabeza promedio (arriba) y el modelo esférico de tres capas (abajo), desde un punto de vista basal. Los tres estímulos diferentes se muestran: $\mathrm{C}$ (izquierda), $\mathrm{C}+\mathrm{P}$ (centro) y $\mathrm{P}$ (derecha).

de SCDP para $\mathrm{C}$ y $\mathrm{C}+\mathrm{P}$ considerando el grupo control fue encontrado en el OFG derecho, mientras que la corteza Fusiforme Temporal-Occipital (TOFC) derecha fue encontrada en caso de utilizar el modelo esférico. Esta diferencia resultó ser aún más notoria para el grupo de pacientes, dado que, considerando los mismos estímulos, el OFG derecho fue encontrado cuando se utilizó el modelo de cabeza realista, mientras que el OFG izquierdo fue encontrado en caso de utilizar el modelo esférico. Resultados similares se obtuvieron al considerar estímulos de P, en donde el OFG izquierdo fue encontrado en todos los casos (con distintas zonas de activación secundarias). Sin embargo, debe notarse que valores de SCDP mayores fueron encontrados cuando el modelo realista fue utilizado, por lo que los resultados con este modelo resultan más fidedignos. 


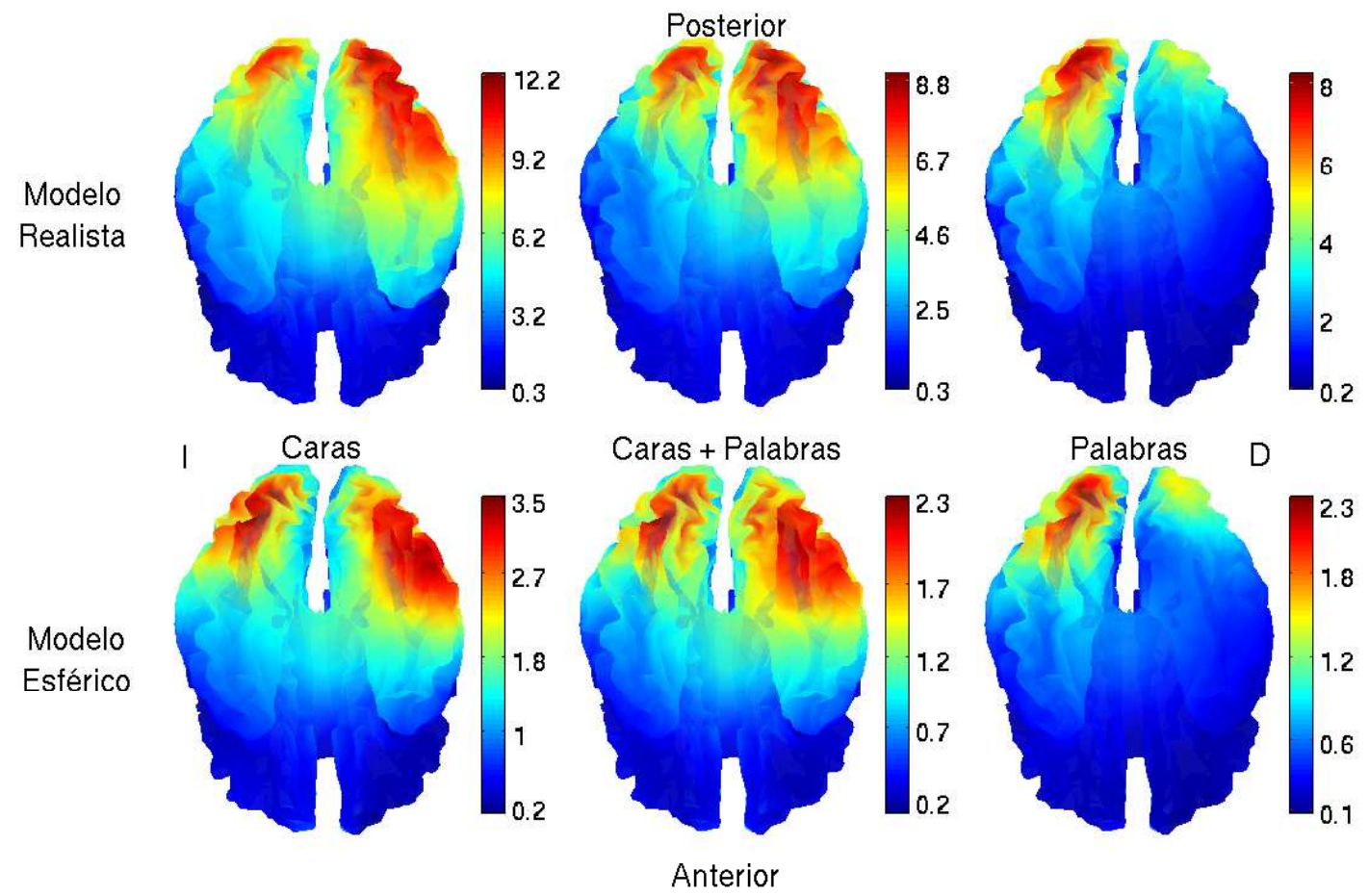

Figura 3.9: $\operatorname{SCDP}\left(\times 10^{-6}\right)$ para pacientes con TDAH. Se muestran resultados considerando el modelo de cabeza promedio (arriba) y el modelo esférico de tres capas (abajo), desde un punto de vista basal. Los tres estímulos diferentes se muestran: C (izquierda), C+P (centro) y P (derecha). 


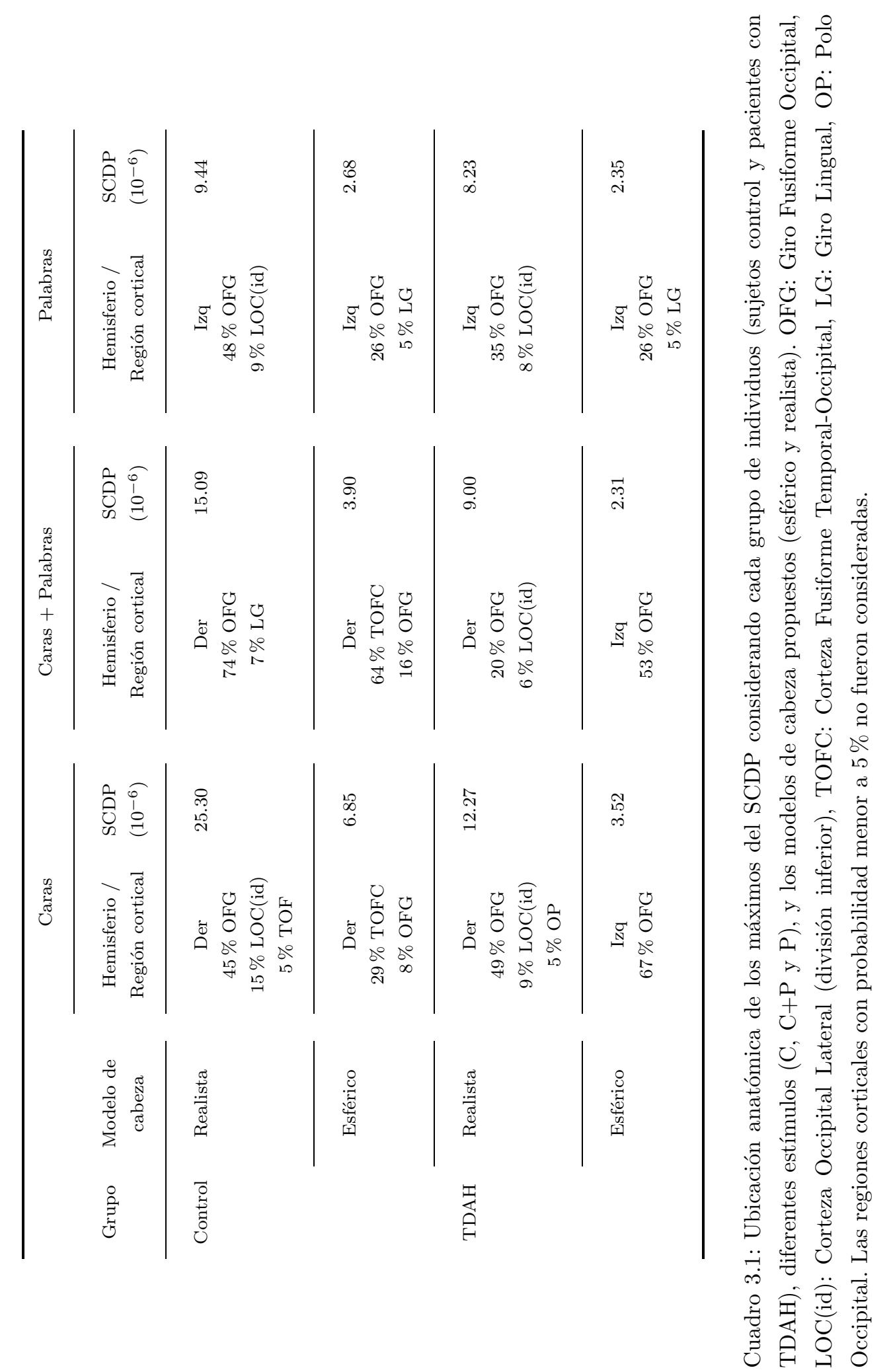




\subsection{Discusión y conclusiones}

El objeto del presente capítulo fue estudiar el impacto del modelo de cabeza en la localización de fuentes asociadas a PE, constituyendo una aplicación directa de los conceptos desarrollados en el Capítulo 2. El análisis de este factor resulta de suma importancia a fin de explicar, en parte, la heterogeneidad de resultados de localización disponibles en la literatura. En particular se analizó la componente N170 considerando C, C+P y P como estímulos, en sujetos de control y pacientes con TDAH.

Con la finalidad de estudiar la influencia del modelo de cabeza en la resolución del PI se consideraron dos modelos. El primero fue el esférico de tres capas, cada una con conductividad eléctrica isótropa y homogénea. Este modelo es utilizado en la mayoría de los trabajos que tienen como eje el análisis de las fuentes que originan a la componente N170, debido a su simplicidad y disponibilidad en un gran número de aplicaciones informáticas de neurociencias (Schweinberger et al., 2002; Watanabe et al., 2003; Herrmann et al., 2005; Santesso et al., 2008; Corrigan et al., 2009). El segundo modelo considerado fue un modelo promedio realista basado en los atlas ICBM- 152 e ICBM- DTI- 81, en donde la anisotropía y heterogeneidad de la conductividad eléctrica del cerebro fue tenida en cuenta. Considerando el método ampliamente aceptado sLORETA se evaluó la varianza de la densidad de corriente reconstruida, la cual permitió comparar la influencia de ambos modelos en la localización de la fuente mediante la relación de varianza. Éste resulta ser un método eficiente dado que depende principalmente de los modelos de cabeza considerados. Los resultados muestran que la varianza en la localización de las fuentes es mayor en el caso de considerar el modelo esférico, especialmente en la TOC, en donde la componente N170 suponía encontrarse. Estos resultados son acordes a los de Vatta et al. (2010), en donde se compara al modelo esférico del realista individual del sujeto.

Sin embargo, puede verse que algunas áreas corticales presentaron una relación de varianza menor a la unidad, lo cual significa que el modelo esférico introduce menor varianza al estimador de la densidad de corriente que el modelo promedio. 
Estas regiones (menores al $20 \%$ del total) resultaron ser mesiales, cercanas al cuerpo calloso. Las razones pueden deberse a múltiples factores. En primer lugar, y como se muestra en Vanrumste et al. (2002), la mejora del modelo realista sobre el modelo esférico en la solución del PI para un sujeto individual resulta ser despreciable en regiones mesiales cuando se considera ruido correlacionado espacial y temporalmente, y un número de electrodos mayor a 44, lo cual sucede en el presente análisis. En segundo lugar, los modelos aquí utilizados son ambos aproximados al real y, por lo tanto, sesgados. Este no es el caso de Vanrumste et al. (2002), en donde los autores consideran a la geometría verdadera conocida. Luego, en ciertos casos puede darse que el modelo promedio diste del modelo individual más que del esférico, justificando estos resultados. De todos modos debe destacarse que las regiones mesiales se descartan del análisis de activación cortical de PE por su ubicación.

En el presente capítulo se consideró el método de sLORETA para resolver el PI. Este método se eligió de un modo un tanto arbitrario dado que ha probado ser adecuado en múltiples problemas relacionados a EEG, tales como iEEG (Rullmann et al., 2009; Cho et al., 2011), ECoG (Dümpelmann et al., 2011), y PE (Weber et al., 2005; Utama et al., 2009; Sumiyoshi et al., 2009). La extensión a otros métodos lineales puede realizarse en función de la matriz de resolución (Michel et al., 2009), la cual relaciona a las densidades de corriente estimada y verdadera, por lo que puede ser utilizada para cuantificar la influencia debida al modelo de cabeza utilizado. Sin embargo, un estudio basado en la matriz de resolución dependerá del conocimiento de los modelos individuales de cada sujeto, lo cual excede el propósito de lo aquí planteado. En el futuro se planea focalizar en el estudio de la influencia del modelo mediante dicho método.

Asimismo, debe aclararse que el método presentado es totalmente dependiente del parámetro de regularización $\alpha$ escogido (ver el ApéndiceB.1), por lo que su variación conllevará a cambios en la distribución del SCDP sobre la superficie cortical. Distintos resultados se han obtenido en función de $\alpha$, considerando en el presente $\alpha=0,5$ para ambos modelos. Futuros estudios estarán ligados a formalizar de un mejor modo la elección del parámetro de regularización, lo cual constituye una materia pendiente en todos los métodos en los que se imponen condiciones sobre la 
norma del estimador buscado. Posibles soluciones a este problema se presentan en los Capítulos 6 y 7 de la presente tesis.

La varianza en la localización es también dependiente del modelo de fuente considerado. El más común es el modelo dipolar, el cual es ampliamente asumido en la bibliografía relacionada a la componente N170 (Shibata et al., 2002; Schweinberger et al., 2002; Watanabe et al., 2003; Rossion et al., 2003; Mnatsakanian y Tarkka, 2004; Deffke et al., 2007). Se mostró en el presente capítulo que al considerar el modelo de fuente dipolar la influencia del modelo de cabeza resultó más alto que de considerar un modelo distribuido, más aún en caso de pacientes con TDAH, debido a su baja amplitud y naturaleza de activación bilateral (Ibáñez et al., 2011b). Luego, si el modelo dipolar es considerado, el modelo de cabeza promedio resulta recomendable. Debe destacarse que el modelo dipolar resulta adecuado cuando se considera a la fuente de actividad cerebral lo suficientemente focalizada. Sin embargo, existe evidencia para considerar al N170 un proceso distribuido e interactivo (Vuilleumier y Pourtois, 2007; Corrigan et al., 2009), volviendo a los modelos dipolares fisiológicamente inadecuados.

Mediante el método propuesto y el modelo de cabeza promedio se buscaron las fuentes de la componente N170. Considerando sujetos control dichas fuentes fueron mayormente localizadas en la TOC derecha (OFG, TOFC y LG) considerando estímulos del tipo $\mathrm{C}$ y $\mathrm{C}+\mathrm{P}$, y en la TOC izquierda (OFG, TOFC y LG) cuando estímulos del tipo $\mathrm{P}$ fueron utilizados. Estos resultados se encuentran en concordancia con algunos trabajos que utilizan, a fin de localizar las regiones corticales relacionadas al estímulo, imágenes de RM funcional (Corrigan et al., 2009). Sin embargo, los resultados aquí presentados no se encuentran en total acuerdo a otros trabajos (Schweinberger et al., 2002; Rossion et al., 2003; Deffke et al., 2007), en donde sólo pocas regiones fueron consideradas. Las razones pueden deberse al modelo de cabeza y, principalmente, de fuente utilizado.

Respecto a estímulo de $\mathrm{P}$, los resultados presentados se encuentran en concordancia con estudios previos (Bentin et al., 1999; Rossion et al., 2003), en donde la fuente se localiza mayormente en la TOC izquierda (OFG, TOFC y LG). Asimismo, se encontró una fuerte lateralización izquierda, como se indica en Rossion et al. (2003); 
Koenig et al. (1998). Sin embargo, y de acuerdo a nuestro conocimiento, no existen resultados de localización distribuida relativa a estímulos del tipo P. Finalmente se reportaron áreas de activación para estímulos del tipo $\mathrm{C}+\mathrm{P}$, las cuales resultaron ser iguales que en el caso de considerar C, con una moderada lateralización a la derecha y menor intensidad.

Respecto de los pacientes con TDAH, las fuentes generadoras de la componente N170 se encontraron en las mismas regiones corticales que para los sujetos de control. Sin embargo, dichas fuentes presentaron una menor intensidad y mayor bilateralidad respecto de los sujetos sanos, siendo acorde a los resultados existentes en la literatura (Herrmann et al., 2009). Esta disminución del potencial medido puede causar una estimación desacertada si el modelo de cabeza utilizado es el esférico, pudiendo hasta influir en el lóbulo de origen del PE. Luego, la influencia de los modelos de fuente y cabeza son aún más importantes cuando se analizan pacientes con patologías ligadas a una disfunción en el procesamiento del estímulo bajo estudio (caras y palabras en el presente caso), por lo que el modelo de cabeza realista debe tenerse en cuenta.

Si bien se mostró que la varianza introducida por los modelos de cabeza y fuente es de importancia, no son las únicas fuentes de error en la localización de la fuente relacionada a los PE. Por ejemplo, en muchos trabajos se resuelve el PI considerando una pequeña cantidad de electrodos (no más de 38 en Shibata et al. (2002); Schweinberger et al. (2002); Herrmann et al. (2005); Itier y Taylor (2004); Deffke et al. (2007); Herrmann et al. (2009)) lo cual conlleva a un aumento inevitable de la varianza en la estimación (Ollikainen et al., 2000; Lantz et al., 2003). Luego, con el fin de reducir la varianza originada por la escasa cantidad de electrodos, disposiciones de 64 o más deben de ser consideradas (Lantz et al., 2003).

Por último debe notarse que los resultados sobre la influencia del modelo en la resolución del PI relacionado a PE puede no verse limitado al caso de la componente N170. Los valores que toma el coeficiente de varianza en otras regiones del cerebro, como la corteza somatosensorial, permite evidenciar la ventaja de la utilización del modelo promedio aquí planteado en el estudio de los PE en general. 


\section{Capítulo 4}

\section{Efecto del modelo de cabeza en estudios simultáneos de ECoG y E/MEG}

\subsection{Introducción}

La finalidad última del PI en E/MEG es la localización de las fuentes de actividad cerebral ligadas a mediciones de potencial eléctrico y/o campo magnético de forma no invasiva. La precisión con la que se resuelve este problema se encuentra limitada por diversos factores, como son la actividad cerebral de fondo, el ruido electrónico de los sensores y los efectos aislantes del cráneo, entre otros. Estas limitaciones pueden ser sustanciales en situaciones donde se requiere la mayor precisión, como es el caso de la planificación de una intervención neuro-quirúrgica de resección. Es por ello que frente a la necesidad de localizar las fuentes del mejor modo posible, los neurocirujanos usualmente resuelven utilizar dispositivos de medición (electrodos) intracraneales, de modo tal de mejorar la calidad de las señales adquiridas y obtener mediciones más fidedignas que las obtenidas mediante métodos no invasivos, i.e. E/MEG.

Las mediciones intracraneales consisten en la adquisición de señales por parte 
de electrodos implantados en el interior del cráneo. Existen diversos dispositivos de medición intracraneal según sean necesarios, resultando ser el electrocorticograma (ECoG) y la electroencefalografía de profundidad (sEEG) las más utilizadas. El ECoG (Blount et al., 2008) consiste en la disposición de electrodos en forma de grilla sobre una base de silicona con la finalidad de colocarse directamente sobre la corteza cerebral y estudiar así su actividad eléctrica. En cambio, la sEEG (Lachaux et al., 2003) consiste en la disposición de electrodos sobre una aguja semi-flexible con la finalidad de adquirir mediciones dentro de la corteza cerebral. Las diferencias entre ambas modalidades son tanto en su utilidad como en su implantación. La grilla subdural permite la adquisición de señales en una amplia región cortical, necesitando para su implantación la realización de una craneatomía de dimensiones similares a la de la grilla. En cambio, los electrodos profundos son recomendados para la adquisición de señales originadas en estructuras corticales profundas o subcorticales. Dada su disposición unidimensional, su implantación necesita de la realización de un agujero en el cráneo mucho más pequeño que en el caso de ECoG. Estas técnicas permiten a los neurocirujanos determinar, junto a otros elementos de juicio, la zona que debe extirparse, normalmente mediante una inspección visual de los registros de ECoG y/o sEEG.

Dado que los registros intracraneales involucran la adquisición de potenciales eléctricos locales durante aproximadamente siete días (Tao et al., 2007b; Huiskamp et al., 2010), los investigadores suelen aprovechar la situación para estudiar con mayor detalle el funcionamiento del cerebro y los efectos del modelo sobre las mediciones de E/MEG. Ejemplos de ello son los estudios cuya finalidad es evaluar comportamientos locales de grupos de neuronas (Ulbert et al., 2001), con el objeto de estudiar la pato-fisiología de desórdenes cerebrales (Engel et al., 2011), así como el estudio de modelos corticales fisiológicamente plausibles (Blenkmann et al., 2011). Del mismo modo, mediciones de ECoG en forma simultánea con EEG (Pacia y Ebersole, 1997; Tao et al., 2005, 2007a; Ray et al., 2007; Hashiguchi et al., 2007; van der Loo et al., 2007; Yamazaki et al., 2011) y MEG (Mikuni et al., 1997; Oishi et al., 2002) se realizan con la finalidad de comparar ambas modalidades y evaluar la confiabilidad y limitaciones de los métodos no invasivos. Dentro de éstos existen 
quienes utilizan dichas señales de ECoG y E/MEG con el objetivo de caracterizar los efectos del cráneo en la medición de señales vinculadas a epilepsia y relacionar a la mínima espiga detectable en el cuero cabelludo con el área de corteza involucrada (Mikuni et al., 1997; Oishi et al., 2002; Tao et al., 2005, 2007a; Ray et al., 2007; Hashiguchi et al., 2007). Diversos factores son encontrados como causantes de la variación de señales medidas con ECoG y E/MEG, tales como la extensión del área cortical involucrada, la amplitud de la descarga, el grado de sincronización neuronal y la región cortical en donde se encuentra la lesión. Sin embargo, en dichos trabajos no se han considerado los efectos aislantes del sustrato no conductor de la grilla subdural ni los de los huecos en el cráneo, pudiendo éstos llegar a ser determinantes en la amplitud y distribución del potencial eléctrico y el campo magnético medido. En el presente capítulo se analiza la importancia de la adecuada consideración del modelo de cabeza utilizado en estudios de ECoG y E/MEG simultáneos. Utilizando el MEF desarrollado en el Capítulo 2 se comparan las soluciones del problema directo haciendo uso de un modelo de cabeza realista (que contemple tanto la grilla subdural como los huecos en el cráneo) y simplificaciones del mismo.

Numerosos trabajos han focalizado en el estudio de la influencia del modelo post quirúrgico en señales de E/MEG. En muchos de ellos (van den Broek et al., 1998; Benar y Gotman, 2002; Flemming et al., 2005; Li et al., 2007) se analiza el efecto de los huecos en el cráneo, concluyendo que los mismos son locales, mayores a medida que la fuente se encuentre más cerca de ellos. En Zhang et al. (2006a) se estudia la influencia de la grilla en mediciones de EEG sobre el cuero cabelludo, resaltando la importancia de su consideración. Sin embargo, ninguno de ellos analiza el efecto conjunto de los huecos y la grilla sobre mediciones de E/MEG, así como tampoco cuantifican los efectos de amplificación/atenuación del modelo en mediciones realizadas por dichas modalidades. En este capítulo se estudian los efectos debidos a las alteraciones del modelo prequirúrgico considerando fuentes dipolares restringidas anatómicamente. Se estudia además la importancia de la adecuada consideración de la grilla subdural y los huecos en el cráneo en estudios de reconstrucción de fuentes basado en señales de ECoG solamente, los cuales rara vez son tenidos en cuenta (Korzyukov et al., 2007; Dümpelmann et al., 2009; Kim et al., 2010; Tanaka 
et al., 2010; Cho et al., 2011; Dümpelmann et al., 2011).

La mayor ventaja de los estudios invasivos respecto de los no invasivos radica en la mejora de la resolución espectral y espacial, así como en la calidad de señal medida (Shenoy et al., 2008), lo que suele cuantificarse mediante la relación señal a ruido (SNR). Este parámetro suele ser determinante en los problemas de detección y clasificación (Bast et al., 2006), por lo que la adecuada caracterización del mismo considerando las diferentes modalidades de medición resulta de notable interés. En Ball et al. (2009) se computa la SNR entre señales de EEG y ECoG adquiridas en forma simultánea, remarcando la importancia de la invasividad del estudio en la calidad de la medición. Sin embargo, en dicho estudio no se considera la variación de la SNR de acuerdo a la posición de la fuente relativa a la grilla aislante, por lo que las razones de la variación resultan ser un tanto difusas. Aquí se utilizan los modelos realizados para comparar la SNR entre señales de E/MEG y ECoG simultáneos, así como la variación de dicho parámetro en modelos pre y post quirúrgicos. Los resultados son de sumo interés en neurociencias, pues permiten cuantificar la ganancia en la calidad de señal obtenida a partir de estudios invasivos.

El presente capítulo se organiza del siguiente modo: en la Sección 4.2 se presenta el mecanismo desarrollado para la obtención de los modelos de cabeza utilizados, así como su discretización y resolución del problema directo mediante el MEF. En la Sección 4.3 se muestran resultados correspondientes a las simulaciones efectuadas, las cuales permiten caracterizar adecuadamente tanto las influencias del modelo en la distribución y magnitud de señal así como de SNR. Finalmente, en la Sección 4.4 se presenta una discusión de los resultados y su implicancia en los estudios propios del estado del arte.

\subsection{Métodos}

En la siguiente sección se explica la metodología desarrollada para la obtención de los modelos de cabeza buscados y la posterior resolución del problema directo. Asimismo, se detallan los parámetros de cálculo que permiten determinar la influen- 
cia del modelo en la obtención del potencial eléctrico y el campo magnético.

\subsection{1. $\quad$ Modelo de cabeza}

Con el objetivo de evaluar los efectos del modelado de la cabeza se utilizaron datos reales de un paciente con epilepsia focal cortical en el lóbulo frontal, refractaria a la medicación. Dicho paciente fue evaluado para resección de la zona dañada utilizando diversos estudios, los cuales incluyeron la utilización de una grilla intracraneal para lograr una mejor descripción de la zona epileptógena. La grilla subdural utilizada consiste en una lámina cuadrada de silicona de $3 \mathrm{~mm}$ de espesor y $8 \mathrm{~cm}$ de lado, conteniendo un total de 64 electrodos dispuestos sobre un trazado cuadricular. La misma fue ubicada con la finalidad de cubrir zonas candidatas a resección descubiertas mediante otras modalidades pre-quirúrgicas.

Durante el estudio invasivo se adquirieron imágenes de RM y de tomografía axial computarizada (TAC), los cuales permitieron la correcta formulación del modelo de la cabeza utilizado. En principio, las imágenes de RM y TAC fueron corregistradas mediante el utilitario SPM8 ${ }^{1}$. Utilizando la imagen de TAC se extrajeron manualmente máscaras correspondientes al cuero cabelludo y el cráneo, así como a la posición de los electrodos de la grilla. Dado que la extracción de la máscara correspondiente a la grilla no fue lo suficientemente adecuada, se corrigió la misma utilizando la ubicación de los electrodos y se suavizó mediante el uso de armónicos esféricos. Luego, basado en la imagen de RM, se extrajo la máscara de líquido cefalorraquídeo (LCR) mediante la herramienta de segmentación probabilística disponible en SPM8. La consideración de este tejido resulta de notable importancia en el modelo, como se muestra en Wolters et al. (2006); Wendel et al. (2008).

Cabe destacar que los segmentadores convencionales, tales como los incluidos en los programas FSL y BrainSuite, no mostraron una adecuada segmentación del dominio debido a la arbitraria posición de los huecos en el cráneo, considerándolos como errores en las imágenes y no como variaciones del modelo.

\footnotetext{
${ }^{1}$ Disponible en http://www.fil.ion.ucl.ac.uk/spm/
} 


\subsubsection{Problema directo}

Una vez segmentado el dominio se procedió al mallado correspondiente para la resolución del problema directo mediante el MEF. Para ello se consideraron tres modelos de cabeza posibles. En el primero (modelo 1) se consideró el modelo de una forma totalmente realista, en donde tanto la grilla subdural como los huecos en el cráneo fueron tenidos en cuenta. En el segundo (modelo 2) se consideraron solamente los huecos en el cráneo, pero no así la grilla subdural. Por último, en el tercero (modelo 3) se simplificó aun más el problema y se consideró un modelo sin huecos en el cráneo ni grilla subdural. La determinación de estos modelos y su posterior comparación permiten determinar la influencia de la consideración de los huecos y la grilla en la resolución del problema directo y evaluar el desempeño de los modelos menos realistas.

Para cada uno de los modelos se discretizó el volumen utilizando elementos tetraédricos obtenidos mediante la aplicación ISO2Mesh ${ }^{2}$ (Fang y Boas, 2009), y corregidos utilizando MeshLab ${ }^{3}$. La malla resultante consistió en aproximadamente 800.000 elementos para cada modelo, con un tamaño promedio de elemento menor a $1 \mathrm{~mm}$ de lado, refinada aún más en las proximidades de las superficies internas. Las conductividades eléctricas consideradas para el cuero cabelludo, cráneo, LCR y cerebro fueron isótropas y homogéneas de valores $0,33 \mathrm{~S} / \mathrm{m}, 0,0093 \mathrm{~S} / \mathrm{m}, 1,79 \mathrm{~S} / \mathrm{m}$ y 0,33 S/m, respectivamente (Baumann et al., 1997; Dannhauer et al., 2010). Dado que el modelo 1 presenta la grilla subdural, y ésta es no conductora, se consideró apropiado eliminar los elementos internos a la misma, resultando ser la grilla un hueco dentro del modelo. En la Figura 4.1 se muestra un corte coronal del mallado correspondiente al modelo 1 , en donde se pueden observar los distintos tejidos considerados, así como la grilla subdural y dos huecos del cráneo.

Por último se resolvió el PD en E/MEG utilizando el MEF desarrollado en el Capítulo 2. Las posibles fuentes de corriente fueron ubicadas en la superficie cortical con momento dipolar normal a la interfaz entre materia blanca y gris (Goldenholz et al., 2009). Para obtener las mallas triangulares de la superficie cortical e interfaz

\footnotetext{
${ }^{2}$ Disponible en http://iso2mesh. sourceforge.net/cgi-bin/index.cgi

${ }^{3}$ Disponible en http://meshlab. sourceforge.net/
} 


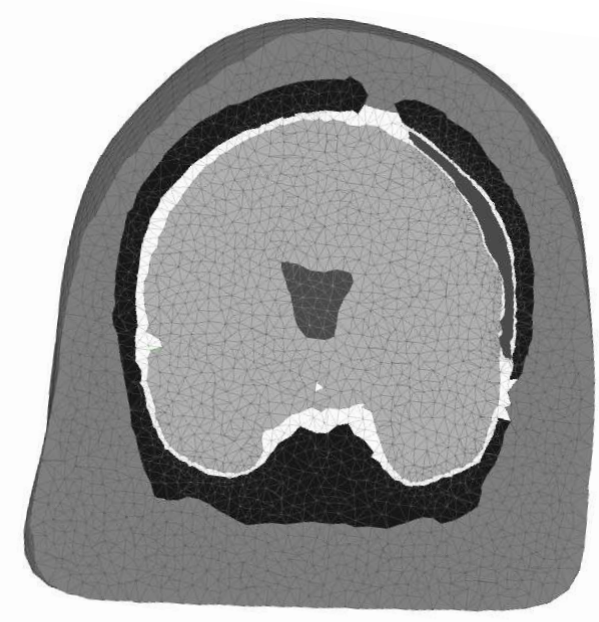

Figura 4.1: Corte coronal del mallado correspondiente al modelo 1. Pueden apreciarse los diferentes tejidos considerados, así como la grilla subdural y dos huecos del cráneo.

de materia blanca-gris se utilizó el programa Freesurfer ${ }^{4}$. En la Figura 4.2 se muestra el modelo resultante desde un punto de vista lateral (a.) y superior (b.), en donde se evidencian las posiciones relativas entre los huecos, la corteza y la grilla.

\subsubsection{Cuantificación de la influencia del modelo}

Una vez resuelto el PD deben definirse parámetros que permitan la adecuada cuantificación de la influencia del modelo sobre el mismo. Diversos factores pueden calcularse en función de los resultados, dependiendo éstos de lo que se quiera estudiar. En este trabajo se consideraron de interés las variaciones tanto en la distribución y magnitud de potencial eléctrico y campo magnético como en la calidad de la señal medida debido a la consideración errónea del modelo.

\footnotetext{
${ }^{4}$ Disponible en http://surfer.nmr.mgh.harvard.edu/
} 

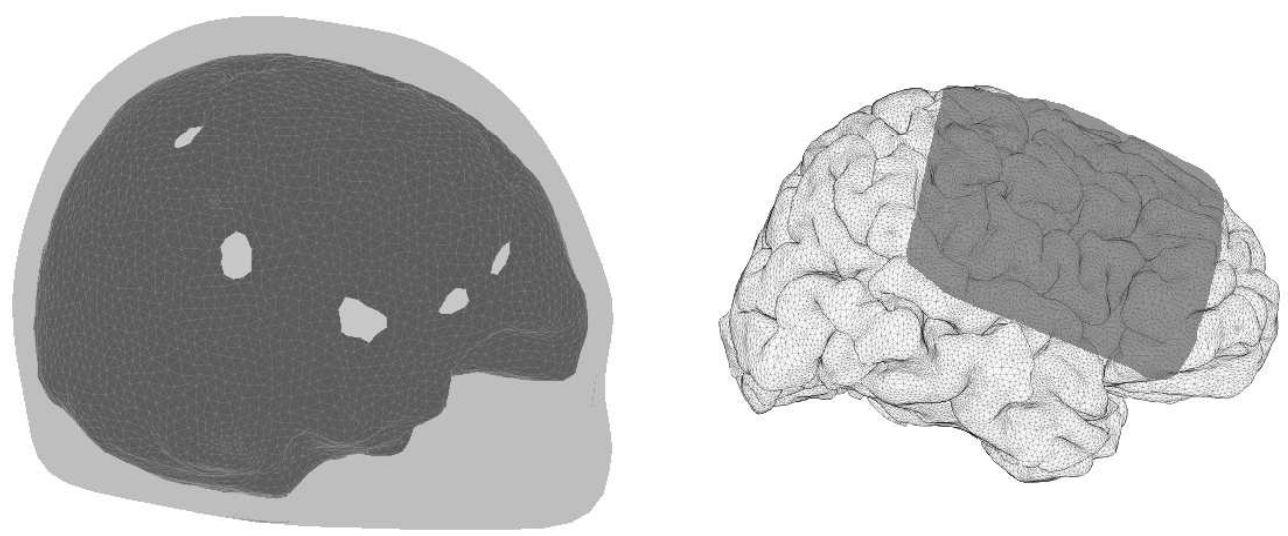

a.
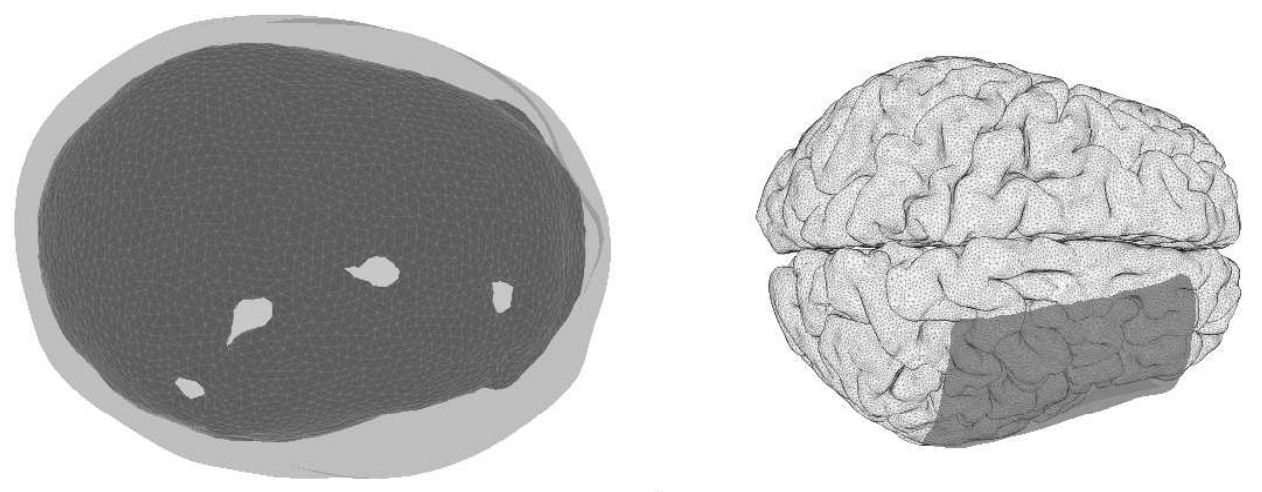

b.

Figura 4.2: Vistas lateral (a.) y superior (b.) del modelo realista utilizado. Pueden observarse las posiciones relativas entre los huecos, la corteza y la grilla.

\section{Variaciones de potencial eléctrico y campo magnético debidas al modelo}

Con la finalidad de evaluar la influencia del modelo en la solución del problema directo es común calcular las variaciones tanto en la distribución espacial como en la amplitud de señal, para lo cual se considera el cómputo de los parámetros NRDM y MAG definidos en el Capítulo 2. Dado que en el presente caso se presumen variaciones locales de ambos factores, se considera adecuado el cálculo de $N R D M_{\infty} \mathrm{y}$ $M A G_{\infty}$. Asimismo, se consideró de interés el cálculo del error normalizado $R D M_{\infty}$, a partir del cual se pueden establecer comparaciones directas entre los errores de potencial eléctrico y campo magnético debidos al modelo en relación a las mediciones obtenidas. 
Los parámetros anteriormente definidos permiten evaluar, de una forma u otra, los errores cometidos en la resolución del PD considerando fuentes sobre la superficie cortical. Sin embargo éstos no posibilitan cuantificar la variación espacial en las mediciones de E/MEG sobre el cuero cabelludo y/o el casco, respectivamente. Esta cuantificación podría resultar de interés para realizar un adecuado posicionamiento de los electrodos sobre el cuero cabelludo a fin de realizar estudios de EEG y ECoG simultáneos. Con este objetivo se propone adaptar los coeficientes anteriores a fin de tener en cuenta dichas variaciones.

La sensibilidad de los electrodos (o bobinas) frente a variaciones del modelo dependerá de la región cortical en donde se consideren ubicadas las fuentes de interés, denominada región de interés (ROI) $\Omega$. En el presente caso, $\Omega$ corresponde a la región de corteza ubicada por debajo de la grilla, asociada a $N_{f}$ nodos de la corteza. Luego, se definen los siguientes factores de mérito

$$
\begin{gathered}
s N R D M^{a b}(\Omega)=\left\|\frac{\phi_{i}^{a}(\Omega)}{\left\|\phi_{i}^{a}(\Omega)\right\|_{2}}-\frac{\phi_{i}^{b}(\Omega)}{\left\|\boldsymbol{\phi}_{i}^{b}(\Omega)\right\|_{2}}\right\|_{2}, \\
s M A G^{a b}(\Omega)=\frac{\left\|\boldsymbol{\phi}_{i}^{a}(\Omega)\right\|_{2}}{\left\|\boldsymbol{\phi}_{i}^{b}(\Omega)\right\|_{2}} \\
\operatorname{sRD} M^{a b}(\Omega)=\frac{\left\|\boldsymbol{\phi}_{i}^{a}(\Omega)-\boldsymbol{\phi}_{i}^{b}(\Omega)\right\|_{2}}{\left\|\boldsymbol{\phi}_{i}^{b}(\Omega)\right\|_{2}}
\end{gathered}
$$

en donde $\phi_{i}^{a}$ es el vector de $N_{f}$ elementos que contiene el potencial eléctrico (o campo magnético) en el i-ésimo sensor (o punto sobre la superficie del cuero cabelludo/casco) debido a cada una de las $N_{f}$ fuentes de la ROI, considerando el modelo $a$ (la definición de $\phi_{i}^{b}$ es análoga considerando el modelo $b$ ). Estos factores permiten determinar, de forma análoga al NRDM, MAG y RDM, la influencia media en la resolución del PD debida a la variación del modelo para fuentes ubicadas en la ROI.

\section{Variaciones de la calidad de señal}

Con el objetivo de evaluar la calidad de la señal y la influencia del modelo en ella consideramos calcular la variación de la SNR. Este parámetro actúa como factor 
de calidad que permite cuantificar la mejora o el empeoramiento en las mediciones debido a la incorporación de la grilla subdural y la consecuente variación del modelo (Ball et al., 2009). Asimismo, es un parámetro de gran importancia en epilepsia, pues las señales utilizadas para su análisis (denominadas espigas) deben exhibir una SNR lo suficientemente alta como para distinguirse del ruido de fondo (Cobb, 1983; Iwasaki et al., 2005) y permitir localizar la fuente en forma precisa (Mosher et al., 1993; Fuchs et al., 1998; Muravchik y Nehorai, 2001; Tarkiainen et al., 2003; Bast et al., 2006).

Para cada fuente de interés sobre la corteza se define la SNR sobre el k-ésimo electrodo como

$$
S N R_{k}=\frac{a_{k}^{2}}{s_{k}^{2}}
$$

en donde $a_{k}$ es la amplitud de la señal de interés y $s_{k}^{2}$ es la varianza de ruido, ambas en el k-ésimo sensor. A partir de esta definición es posible calcular un factor de mérito sobre la SNR de todos los sensores, como el promedio (Goldenholz et al., 2009) o el máximo, siendo este último el aquí elegido. Dado que en este caso sólo se busca comparar los efectos debidos al modelo, los resultados serán comparativos e independientes de la amplitud de fuente de señal considerada.

El ruido de fondo se obtuvo de suponer 400 fuentes con posición aleatoria sobre la interfaz entre la materia blanca y la gris, normalizado de modo tal que el potencial eléctrico máximo utilizando el modelo 3 sea de $6 \mu V$ (un análisis similar se utiliza, por ejemplo, en Dogandžić y Nehorai (2000); Goldenholz et al. (2009)).

\subsection{Resultados}

En esta sección se muestran los resultados correspondientes a los parámetros de cuantificación de error debido al modelo utilizado. En primer lugar, se muestran variaciones en los resultados del PD en E/MEG y ECoG debidos a la utilización de los distintos modelos. Éstos permiten determinar la influencia de la grilla, los huecos, o ambas en la medición de potencial eléctrico y/o campo magnético. Luego se mues- 
tran variaciones en la SNR en E/MEG y se las compara con la obtenida mediante ECoG. La ROI considerada corresponde a zonas de corteza cerebral distantes de la grilla subdural en hasta $1,5 \mathrm{~cm}$.

\subsubsection{Factores de desempeño}

Se muestran ahora resultados concernientes a la variación en la distribución y magnitud del potencial eléctrico (tanto sobre la grilla subdural como sobre el cuero cabelludo) y del campo magnético debidas a la variación del modelo de cabeza utilizado.

\section{EEG}

En primer lugar se procedió a calcular la variación en la distribución y magnitud del potencial eléctrico debida a la utilización de los modelos detallados anteriormente. En la Figura 4.3 se muestran los factores NRDM, MAG y RDM de EEG para fuentes dipolares ubicadas sobre la corteza considerando los modelos 2- 3 (izquierda), 1-2 (centro), y 1-3 (derecha).

En ella pueden evaluarse las diferencias en la distribución y magnitud del potencial eléctrico medido según se consideren la grilla subdural, los huecos en el cráneo, o ambos. De la Figura 4.3 se desprende que los efectos en la distribución (dados por el NRDM) y magnitud (dados por el MAG) del potencial eléctrico son en su mayoría debidos a la disposición de la grilla subdural, tal como puede apreciarse en la comparación entre los modelos 1-2 y 1-3. En ella se vislumbra que la variación en la distribución del potencial aumenta a medida que las fuentes se encuentran más cercanas a los bordes de la grilla, llegando a tomar el NRDM valores de hasta 1,5. Respecto a las variaciones de la magnitud entre los modelos 1-3, éstas aumentan a medida que las fuentes se encuentran más cercanas al centro de la grilla, tomando valores mínimos de hasta 0,03. Esto implica que si una fuente dipolar se encuentra en el centro de la zona de interés justo por debajo de la grilla, el máximo potencial eléctrico medido sobre el cuero cabelludo considerando el modelo 1 puede reducirse hasta 30 veces respecto del potencial eléctrico medido utilizando los modelos 2 ó 3. 

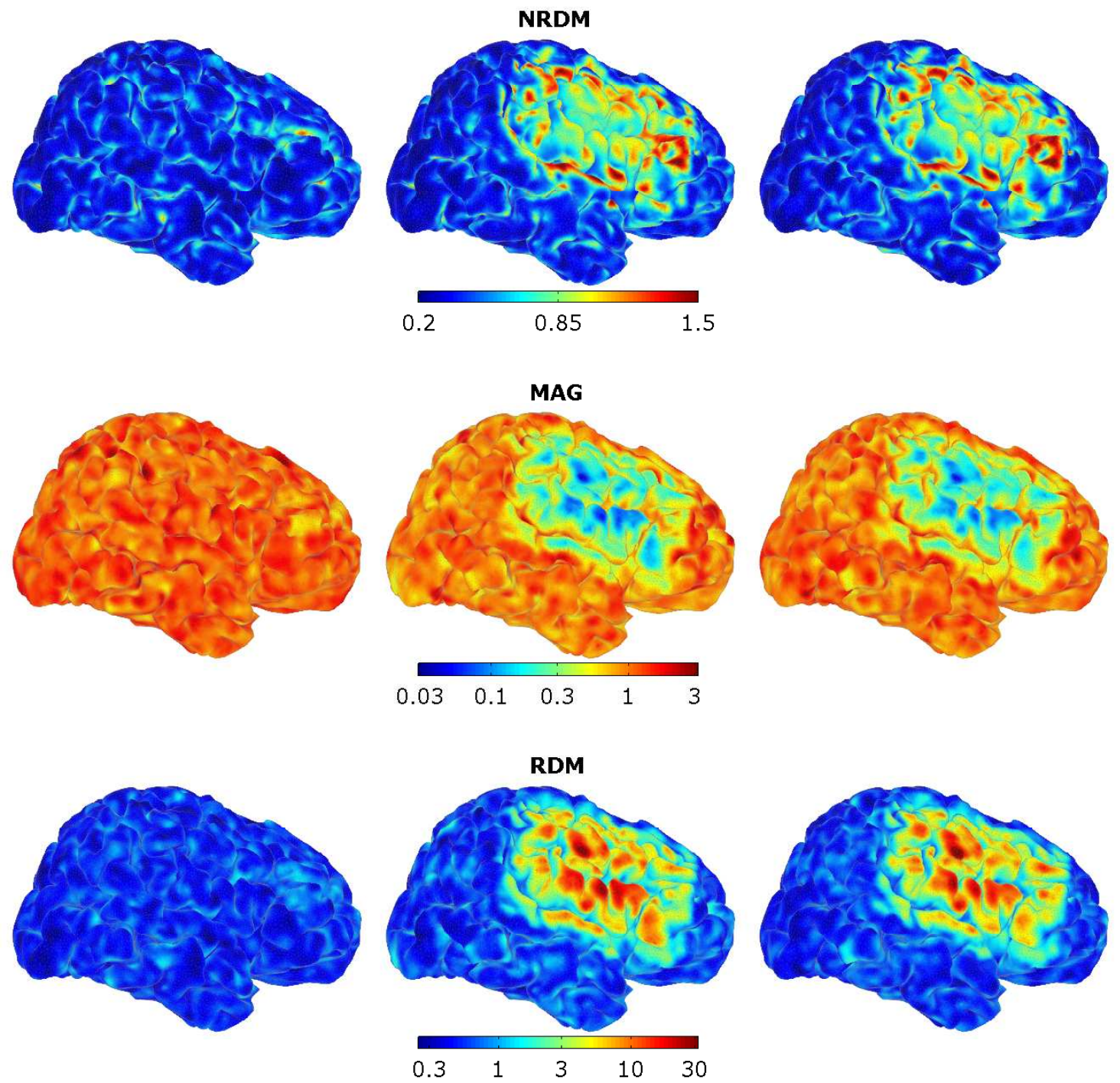

Figura 4.3: Influencia del modelo en el PD de EEG para fuentes dipolares ubicadas sobre la corteza cerebral. Se muestra la variación de NRDM, MAG y RDM considerando los modelos 23 (izquierda), 1-2 (centro), y 1-3 (derecha).

Cabe destacar que los efectos de la grilla subdural son dominantes frente a los de los huecos, como puede verse de la comparación de los factores entre los modelos 2-3 y 1-3, respectivamente. Sin embargo, estos efectos son solo de gran significación para fuentes cercanas a la grilla subdural con momento dipolar normal a ésta. En 
cambio, los efectos locales debidos a los huecos en el cráneo son mayores para fuentes tangenciales cercanas a los mismos, especialmente en el NRDM, en donde supera a la unidad.

Tanto los efectos del NRDM como del MAG se ven comprendidos en el cálculo del RDM, el cual permite cuantificar la influencia relativa del modelo en el potencial eléctrico resultante del PD. En la Figura 4.3 puede verse que el RDM se encuentra fuertemente influenciado por la inclusión de la grilla en el modelo, tomando un valor promedio de aproximadamente 2 en la zona de interés. Debe notarse que, al igual que el MAG, este factor aumenta a medida que la fuente se encuentra mas cercana al centro de la grilla, llegando a tomar un valor máximo de 30. Esto implica que la no consideración de la grilla conlleva a errores del 200 \% (en promedio en la zona de interés).

Cabe destacar que en el caso de considerar los factores $N R D M_{2}, M A G_{2}$ y $R D M_{2}$ los efectos de la grilla se reducen debido al efecto de promediación propio de la norma 2. Dichas variaciones pueden apreciarse en la Figura 4.4, en donde se muestran histogramas de los valores de NRDM, MAG y RDM correspondientes a la comparación entre los modelos 1-3 en la zona de interés, considerando tanto la norma infinito como la norma 2.

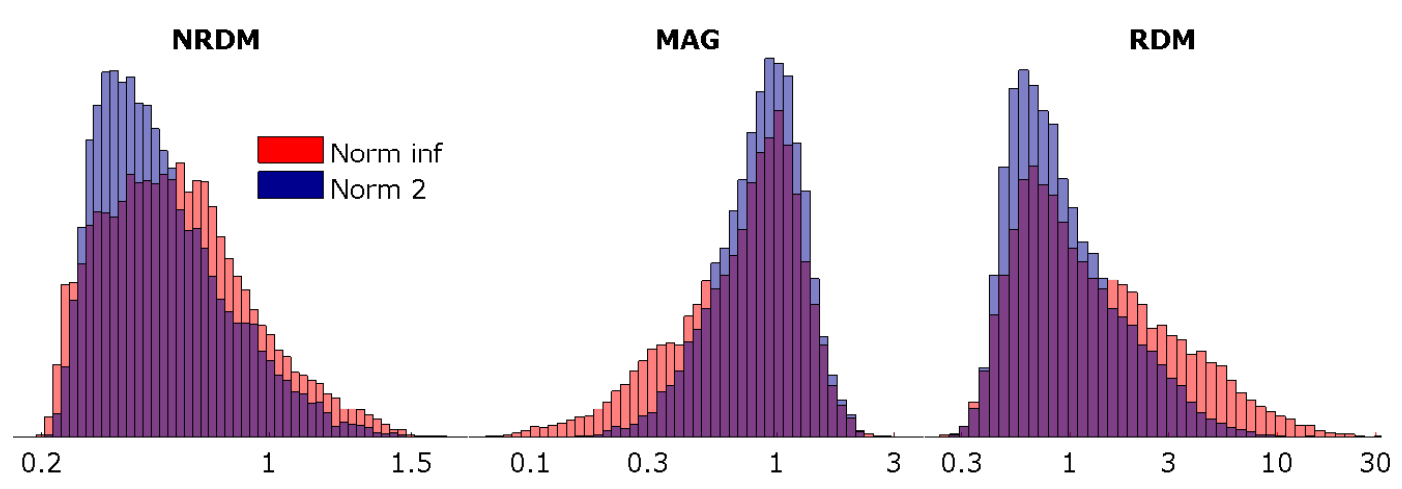

Figura 4.4: Histogramas comparativos del NRDM, MAG y RDM de EEG entre los modelos 1 y 3 , considerando la norma infinito (en rojo) y la norma 2 (en azul).

Se ve claramente que si bien la influencia del modelo tiende a disminuir cuando se utiliza la norma 2, ésta sigue siendo sustancial. Asimismo, pueden notarse (al 
igual que en la Figura 4.3) que para ciertas fuentes el MAG toma valores mayores a la unidad, teniendo lugar éstas en las circunvoluciones vecinas a los huecos.

En la Figura4.5 se muestran los efectos del modelo en las mediciones de potencial medido sobre la superficie del cuero cabelludo, de acuerdo a las expresiones (4.1) - (4.3). Para ello se consideraron posibles fuentes sobre cada nodo de la superficie cortical bajo análisis. Por ende, se muestran las variaciones de potencial sobre el cuero cabelludo debidas a la grilla, los huecos o ambos considerando sólo fuentes en la ROI. La perspectiva utilizada corresponde a la de la Figura 4.2a.
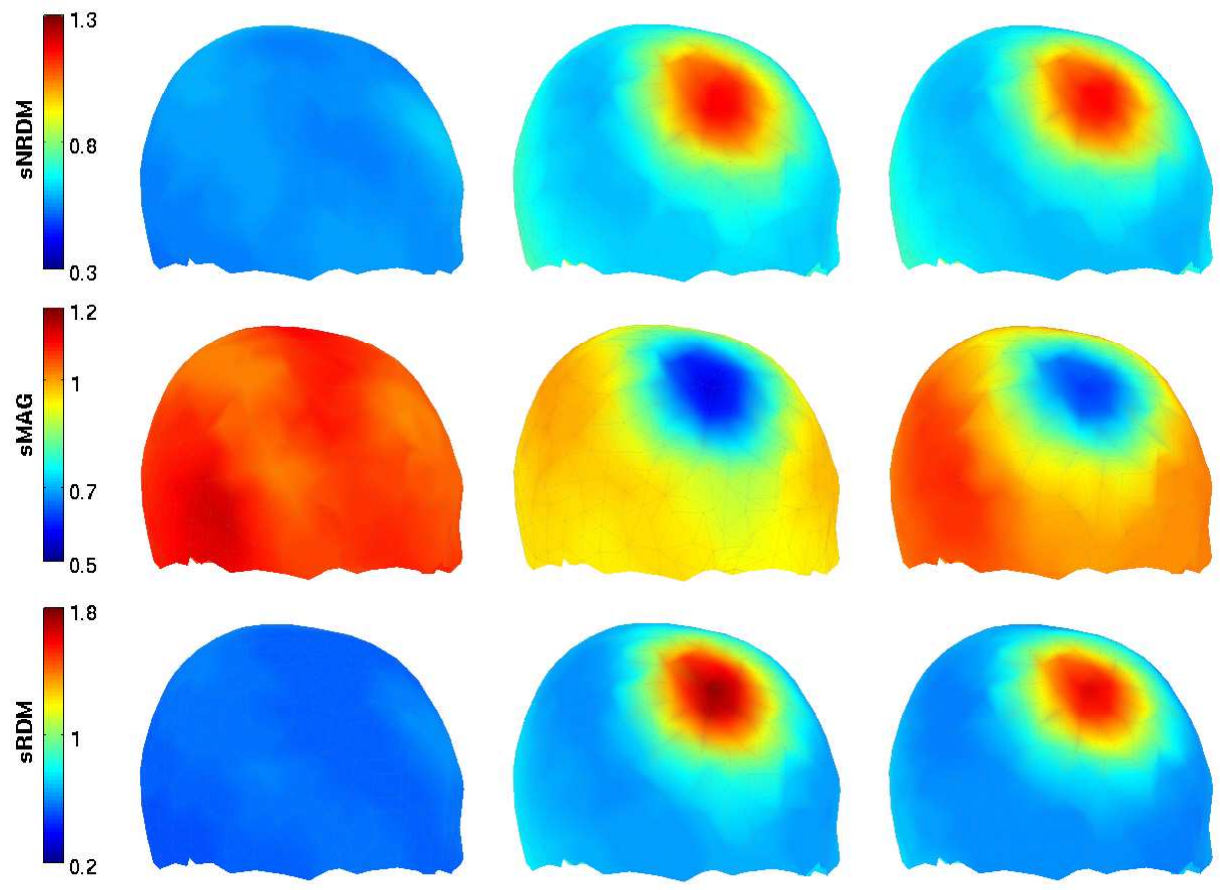

Figura 4.5: sNRDM, sMAG y sRDM sobre el cuero cabelludo para fuentes dipolares en la zona de la corteza bajo estudio. Se muestra este factor considerando los modelos 1-2 (izquierda), 2-3 (centro), y 1-3 (derecha).

Puede verse que los efectos sobre las mediciones son predominantes en aquellos electrodos cercanos a la grilla, para los cuales la distorsión en distribución y magnitud son considerablemente elevados. Es de destacar la variación del MAG para electrodos en dicha zona, en donde llega a tomar el valor de 0,5. Esto implica 
que señales generadas por fuentes ubicadas en la ROI tendrán, en promedio, una disminución del $50 \%$ de amplitud en electrodos ubicados por sobre la grilla. Sin embargo es necesario remarcar que el efecto de los huecos en la amplitud de señal medida (comparación entre los modelos 2-3) tiende a contrarrestar el efecto de la grilla subdural, resultando ser el modelo 3 menos influyente sobre dicho parámetro que el modelo 2. Sin embargo, estos efectos distan de cancelarse mutuamente. Por último, debe notarse que los errores promedio en las mediciones efectuadas por los electrodos mencionados anteriormente es aproximadamente del $160 \%$.

\section{MEG}

Igual procedimiento se aplicó para señales de MEG. En la Figura 4.6 se muestra el NRDM, MAG y RDM de MEG para fuentes dipolares ubicadas sobre la corteza considerando los modelos 2-3 (izquierda), 1-2 (centro), y 1-3 (derecha). En ella puede verse que la distribución de campo normal al casco (dado por el NRDM) varía significativamente con la incorporación de los huecos y la grilla, especialmente en regiones de la corteza cercanas a los bordes de la grilla y los huecos, en donde llega a alcanzar valores de 1,6. Sin embargo, la magnitud de señal (dada por el MAG) no varía significativamente con el modelo utilizado (en comparación al caso de EEG), tomando un valor promedio de 1,07 en la zona de interés, siendo mayor para fuentes tangenciales sobre los surcos de la corteza. Este hecho hace que el error normalizado máximo tome un valor promedio de 0,53 en la región de interés, por lo que la omisión de la grilla en el modelo conllevará a errores máximos del $53 \%$ (en promedio en la zona de interés).

A diferencia del caso de EEG, los resultados prácticamente no varían al considerar la norma 2 para el cálculo de los coeficientes. Esto puede apreciarse en la Figura 4.7, en donde se muestran histogramas de los valores de NRDM, MAG y RDM correspondientes a la comparación entre los modelos 1-3 en la zona de interés, considerando tanto la norma infinito como la norma 2.

Las variaciones del campo magnético calculado respecto del modelo de cabeza utilizado responde a variaciones del campo magnético secundario definido en el Capítulo 2, los cuales son dependientes de la corriente de volumen generada por la 

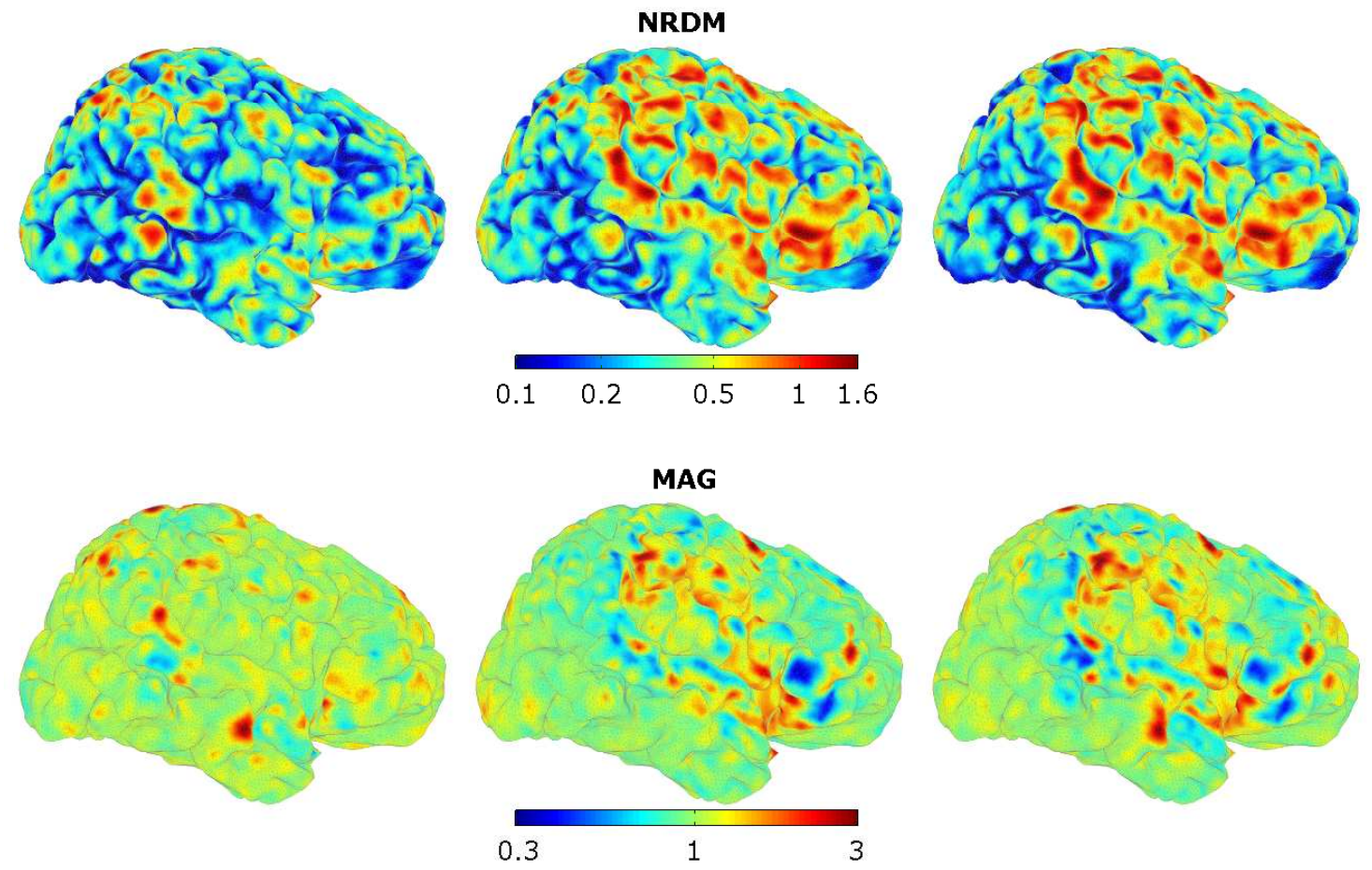

$$
0.3
$$

RDM
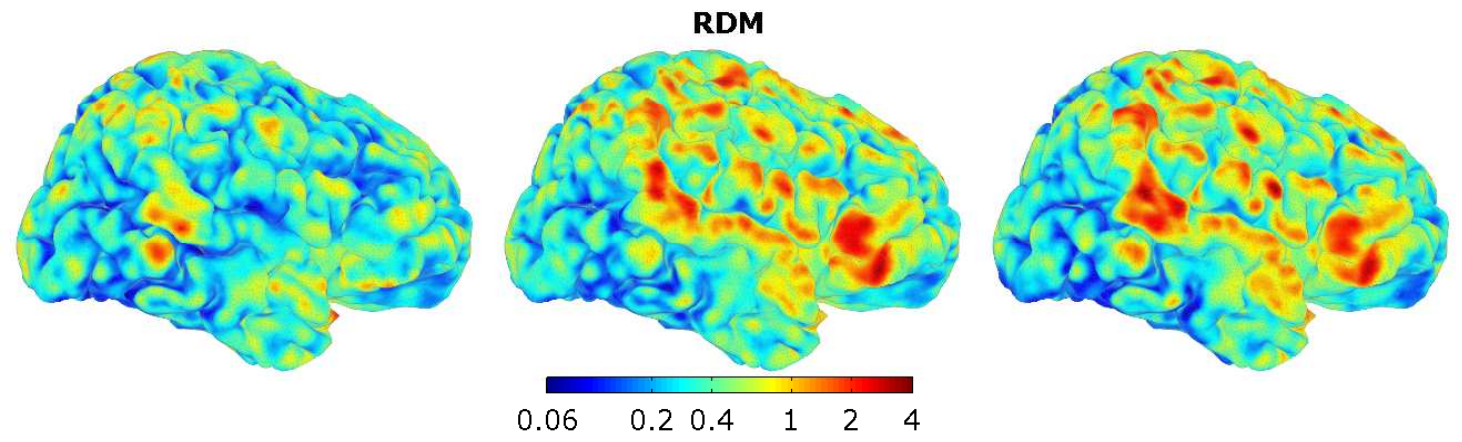

Figura 4.6: Influencia del modelo en el PD de MEG para fuentes dipolares ubicadas sobre la corteza cerebral. Se muestra la variación de NRDM, MAG y RDM considerando los modelos 23 (izquierda), 1-2 (centro), y 1-3 (derecha). Se consideró la medición de la componente normal al casco solamente.

fuente primaria. Al ser ésta dependiente del medio, la incorporación de los huecos y (fundamentalmente) de la grilla en el modelo generarán variaciones en su distribución debido a la naturaleza no conductora del sustrato. Luego, la incorporación de la grilla subdural en el modelo de MEG resulta de vital interés. 


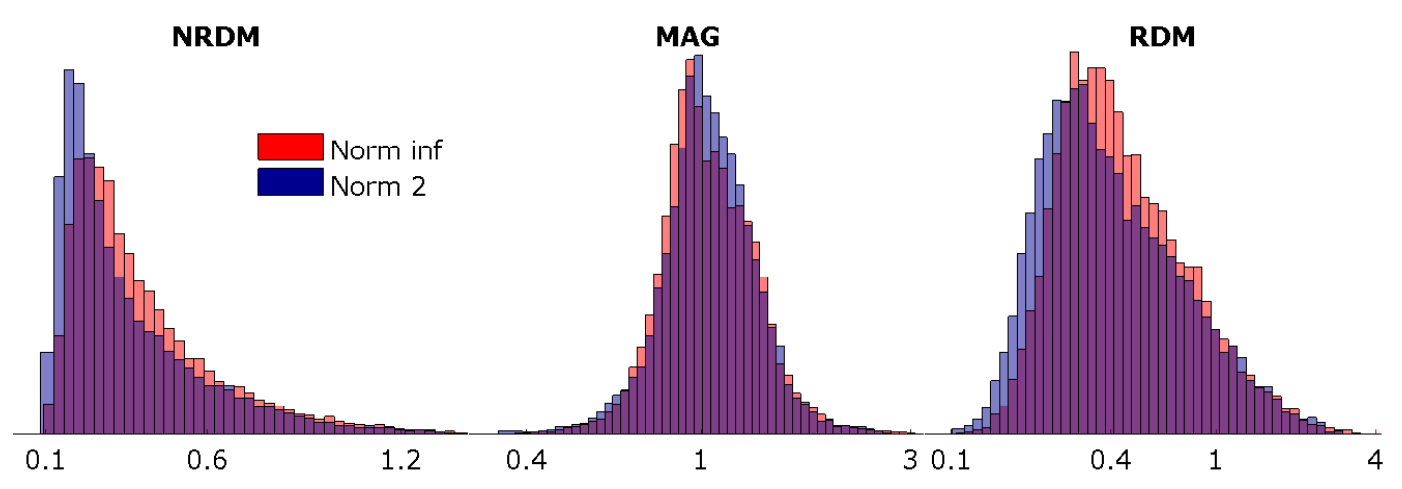

Figura 4.7: Histogramas comparativos del NRDM, MAG y RDM de MEG entre los modelos 1 y 3 , considerando la norma infinito (en rojo) y la norma 2 (en azul).

En la Figura 4.8 se muestra un ejemplo gráfico que permite percibir las variaciones de la densidad de corriente secundaria debido a la incorporación de grilla y huecos en el modelo. Para ello se consideró una fuente dipolar tangencial a la grilla ubicada debajo de ésta, de magnitud 10nAm. Se utilizó la técnica de integral de convolución lineal (LIC) para graficar el campo vectorial correspondiente (Cabral y Leedom, 1993). Puede observarse la variación de la densidad de corriente debida fundamentalmente a la grilla, la que genera una redistribución significativa tanto de su amplitud como de su dirección. Similares influencias se encontraron para fuentes dipolares con orientación normal a la grilla y en distintas posiciones de la ROI.

Finalmente, en la Figura 4.9 se muestran los coeficientes descriptos por las expresiones (4.1) - (4.3) sobre el casco de medición. Al igual que en el caso de EEG, se consideraron solo fuentes en la ROI, por lo que los resultados corresponden solo a fuentes en dicha región cortical. La perspectiva corresponde a la de la Figura 4.2b. Son de remarcar nuevamente los aportes significativos de la grilla a la variación del campo magnético medido, llevando a errores de medición de $50 \%$ en promedio para fuentes ubicadas en la ROI y bobinas en la cercanía de la grilla. Sin embargo debe notarse que dichos efectos son sustancialmente menores que en el caso de EEG, lo cual se ve reflejado en la disminución del sMAG en sólo el 5\% (en las bobinas anteriormente mencionadas). 


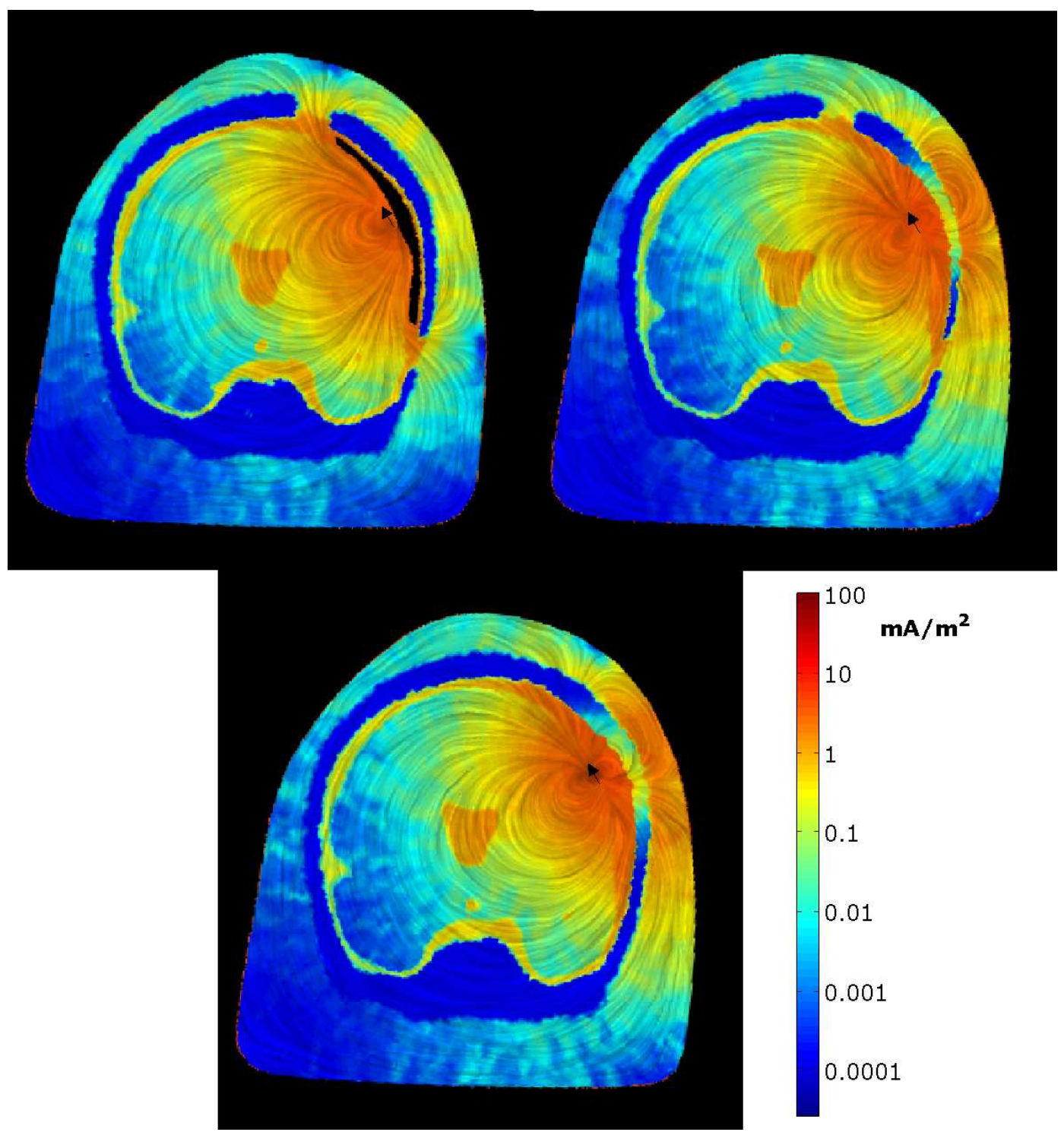

Figura 4.8: Densidad de corriente secundaria debida a una fuente dipolar de 10nAm (marcada por una flecha) considerando los modelos 1 (arriba izquierda), 2 (arriba derecha) y 3 (abajo).

\section{ECoG}

En tercer lugar se estudió la influencia del modelo utilizado en la reconstrucción del potencial eléctrico medido por los electrodos ubicados en el sustrato. Si bien los mismos factores de desempeño utilizados en E/MEG pueden calcularse para este caso, la normalización que conlleva a su cómputo tiende a volver un tanto confusos 

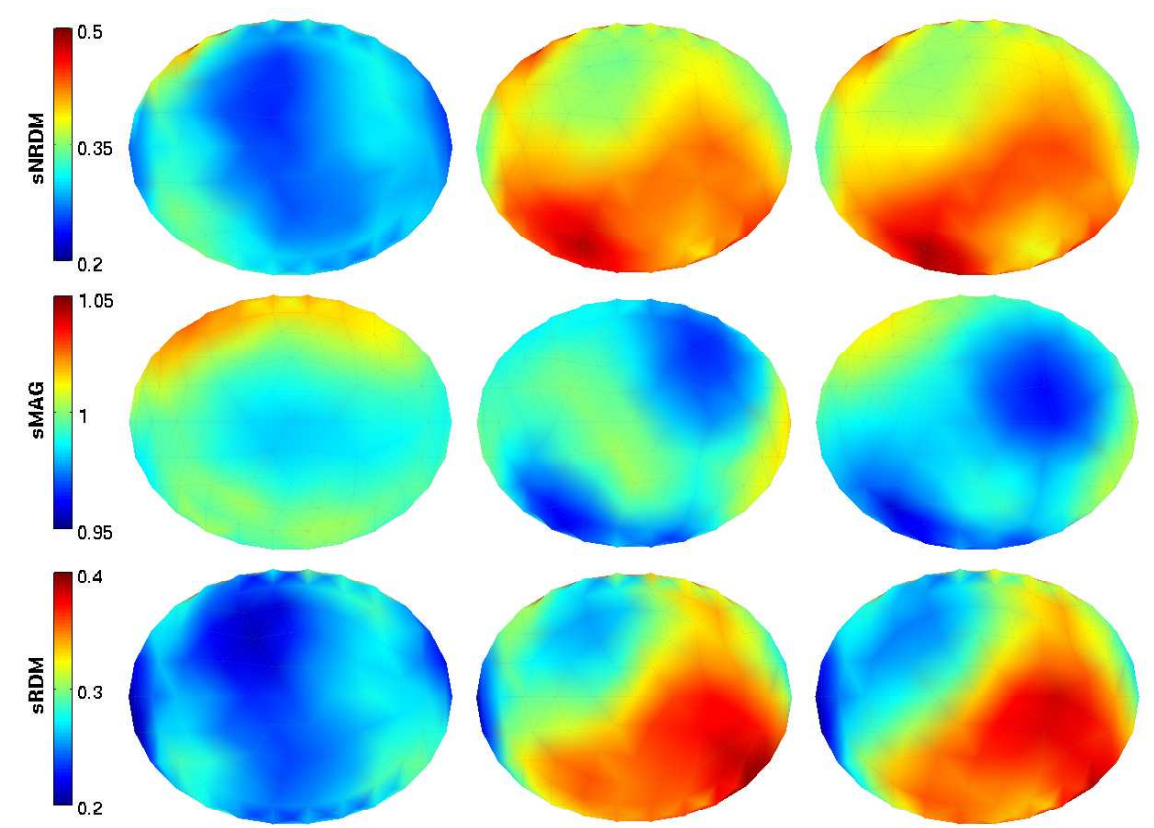

Figura 4.9: sNRDM, sMAG y sRDM sobre el casco de medición para fuentes dipolares en la zona de la corteza bajo estudio. Se muestra este factor considerando los modelos 1-2 (izquierda), 2-3 (centro), y 1-3 (derecha).

los resultados. Esto es debido a que, a diferencia de E/MEG, la distancia de la fuente a los electrodos es altamente variable, razón por la cual errores pequeños pueden resultar en factores de desempeño elevados debida a la baja amplitud de señal. Por ello se consideró válido calcular la norma de la diferencia como factor de interés en las mediciones de ECoG. En la Figura 4.10 se muestra la norma infinito de la diferencia entre los modelos 2-3 (izquierda), 1-2 (centro) y 1-3 (derecha), normalizados todos ellos por el promedio del máximo potencial medido en los 64 electrodos (M).

Puede verse de la comparación de los modelos 2-3 que la influencia de la grilla es considerablemente alta en las vecindades de los electrodos, llegando a tomar tres veces el valor de M. Sin embargo, esta influencia se vuelve 10 veces menor a solo unos pocos milímetros, permitiendo confirmar el efecto local de la grilla sobre las mediciones. Cabe remarcar que, como se ve de la comparación de los modelos 2-3, la influencia de los huecos es considerablemente menor a la de la grilla subdural 

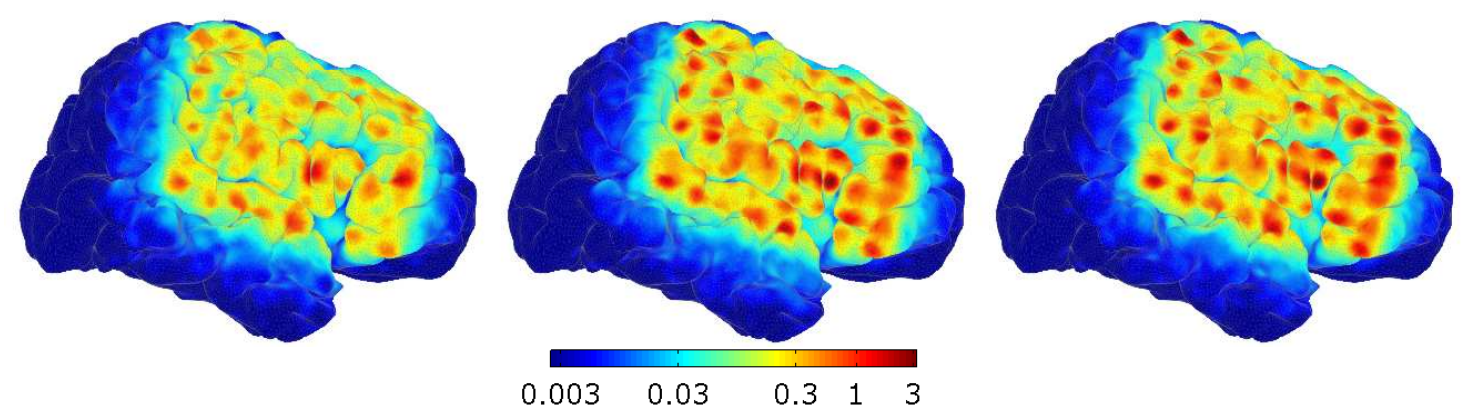

Figura 4.10: Norma infinito de la diferencia entre los modelos 2-3 (izquierda), 1-2 (centro) y 1-3 (derecha). Dichos valores se encuentran normalizados por el promedio del máximo potencial medido en los 64 electrodos.

(entre 5 y 10 veces), por lo que sus efectos resultarán de segundo orden frente a los del sustrato.

Finalmente se procedió a comparar la relación entre las amplitudes de señal máxima obtenidas mediante EEG sobre el cuero cabelludo y ECoG sobre la grilla subdural, es decir, el $M A G_{\infty}$ entre ambos sistemas de medición. Esto permite evaluar la influencia del modelo en la medición simultánea de señales para su análisis cualitativo. En la Figura 4.11 se muestra dicho coeficiente considerando los modelos 1 y 2, desconsiderando al modelo 3 por presentar resultados prácticamente iguales a los correspondientes al segundo. Asimismo, se muestra un histograma que permite evaluar la diferencia de dicho parámetro en la ROI.

De aquí se desprende la necesidad de representar al sustrato no conductor de la grilla en estudios de EEG y ECoG simultáneos, ya que de no considerarla se concluiría que la atenuación sobre la señal máxima es de 8,7 veces (en promedio para fuentes sobre la ROI), cuando en realidad es de 39,4. Esta relación es menor para fuentes más cercanas al borde de la grilla o fuentes más profundas, y mayor para fuentes cercanas al centro del sustrato. 


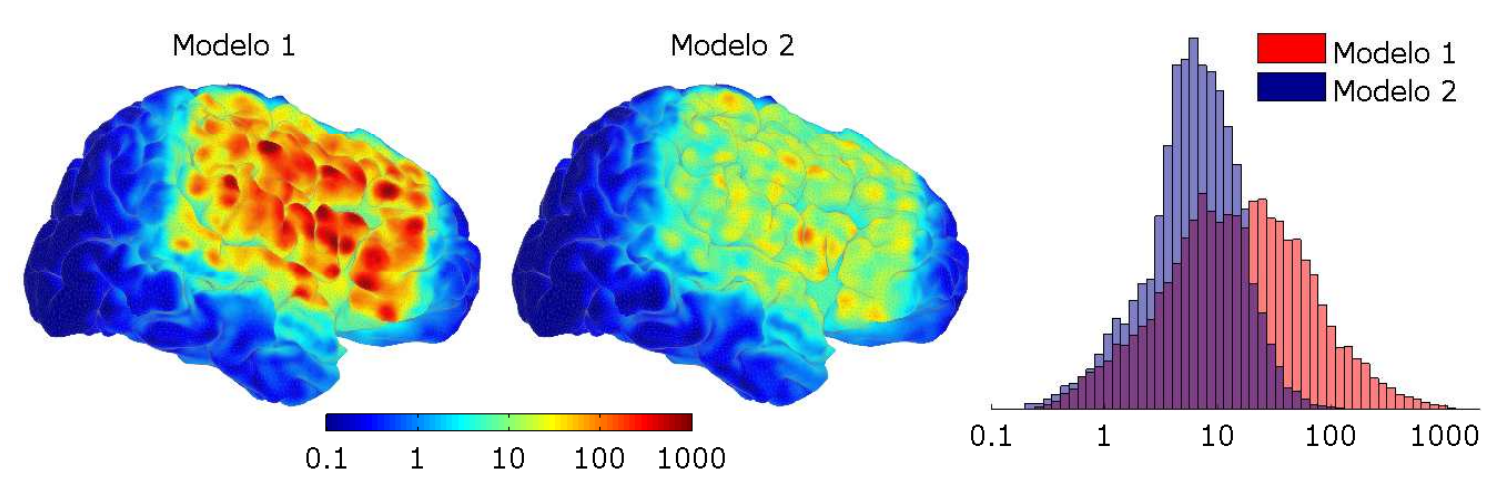

Figura 4.11: Relación entre las amplitudes de señal máxima obtenidas mediante EEG sobre el cuero cabelludo y ECoG sobre la grilla subdural, utilizando los modelos 1 y 2. Asimismo, se muestra un histograma que permite comparar dichos valores en la ROI.

\subsubsection{Variaciones de la calidad de señal}

Por último se procedió a calcular los efectos del modelo en la SNR. En la Figura 4.12 se muestra el cociente entre la SNR de las mediciones de ECoG (utilizando el modelo 1) y la SNR de las mediciones de EEG (considerando el modelo 1 en a. y el modelo 3 en b.) y MEG (considerando el modelo 1 en c. y el modelo 3 en d.). La comparación directa entre las SNR correspondientes a la ECoG y E/MEG considerando el modelo 1 permite estudiar la diferencia existente entre la calidad de señal medida simultáneamente entre dichas modalidades. Por otro lado, la comparación entre las SNR de ECoG y E/MEG utilizando el modelo 3 permite evaluar la mejora en la calidad de señal debida a la disposición de la grilla subdural, es decir, los beneficios del estudio invasivo respecto del no invasivo. Asimismo, se presenta un histograma que permite cuantificar dichas diferencias en la ROI para los casos de EEG (arriba) y MEG (abajo).

Mediante la comparación directa entre las Figuras 4.12a. y 4.12b. puede observarse la influencia del modelo considerado en las mediciones de EEG sobre el cuero cabelludo. En el caso de contar con un modelo realista (modelo 1), la SNR de las señales de ECoG puede llegar a ser hasta 1000 veces mayor a la de las señales de EEG en las inmediaciones del centro de la grilla. Sin embargo, esta relación decrece para fuentes alejadas de ésta, tomando valores entre 10 y 100 (con un valor medio 
a.
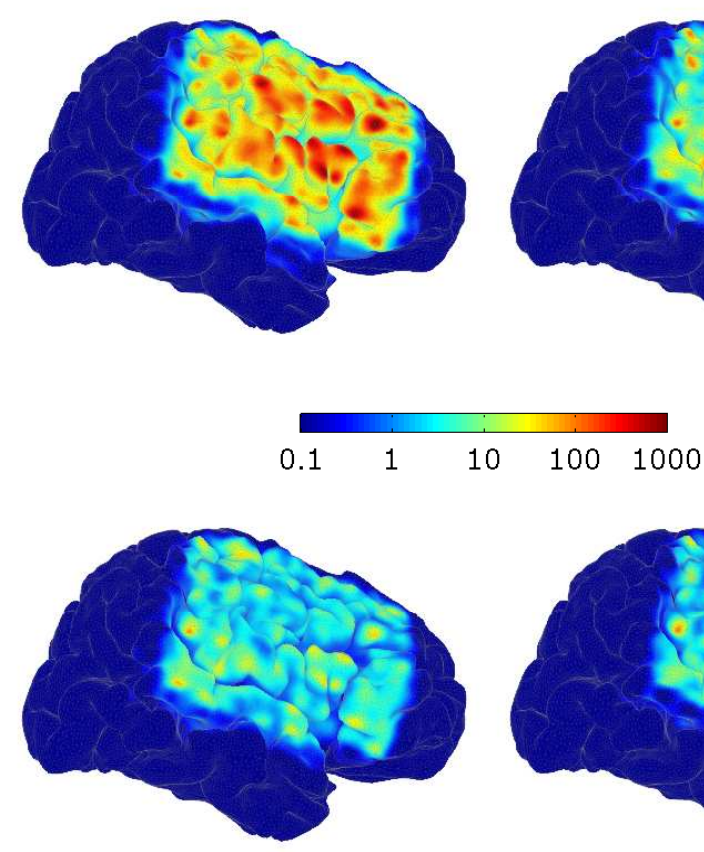

C. b.

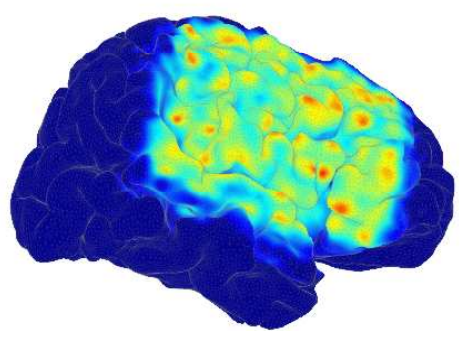

1001000

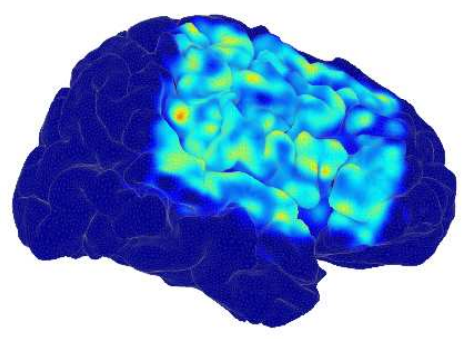

d.
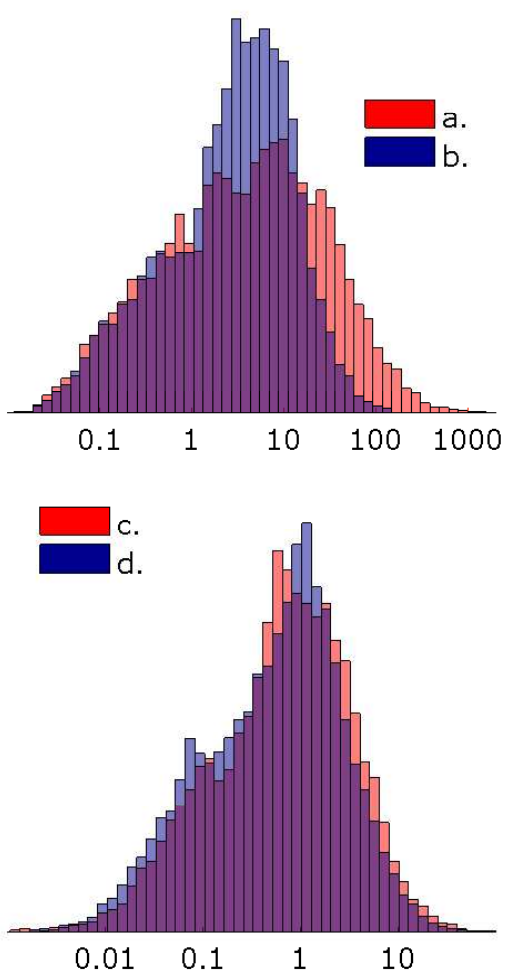

Figura 4.12: Ganancia de SNR de las señales de ECoG respecto de las de EEG y MEG. Se muestra el cociente entre la SNR de las mediciones de ECoG (utilizando el modelo 1) y la SNR de las mediciones de EEG (considerando el modelo 1 en a. y el modelo 3 en b.) y MEG (considerando el modelo 1 en c. y el modelo 3 en d.). Asimismo, se presenta un histograma que permite cuantificar dichas diferencias en la ROI para los casos de EEG (arriba) y MEG (abajo).

de 25) en la zona de interés. Esta relación disminuye si se considera el modelo 3 para las señales de EEG, tomando valores entre 1 y 10 en la zona bajo análisis (con un promedio de 6). Puede notarse que los efectos de los electrodos de ECoG en la SNR son aún más locales que en el caso anterior, llegando a ser 100 veces mayor a la señal medida en EEG justo por debajo de ellos. Luego, el modelo influye de sobremanera en la calidad de señal medida sobre el cuero cabelludo.

Distinto es el caso para mediciones de campo magnético (Figuras 4.12c. y 4.12d.) en donde el modelo no influye de forma tan determinante en la SNR. Si bien la ganancia de SNR de las señales de ECoG respecto a las de MEG se incrementa cuando el modelo 1 es utilizado, ésta no lo hace de forma tan sustancial como en 
el caso de mediciones de EEG. Tanto si se considera el modelo 1 como el modelo 2 los valores de dicha ganancia de SNR oscila entre 1 y 50, tomando el máximo en las inmediaciones de los electrodos subdurales. Sin embargo, el valor medio de esta relación en la zona de interés pasa de ser 4,6 utilizando el modelo 1 a 7,7 utilizando el modelo 3, por lo que la incorporación de la grilla subdural reduce al $60 \%$ la SNR del registro de MEG (en promedio para la ROI).

\subsection{Discusión y conclusiones}

En el presente capítulo se analizó la influencia del adecuado modelado de la grilla subdural y los huecos en el cráneo relacionados a estudios invasivos de ECoG. A partir de la comparación directa entre un modelo realista que considera dichas variaciones con aproximaciones simplistas del mismo se mostraron los efectos en la magnitud y distribución de la señal medida utilizando tanto ECoG como E/MEG. Dichos modelos permitieron también evaluar cambios en la SNR debidas a la variación del modelo.

En primer lugar se estudió la variación en la magnitud y distribución de señal medida mediante E/MEG debida a la incorporación de la grilla y los huecos en el cráneo. Los resultados indicaron que en el caso de señales de EEG los efectos de la grilla son por demás influyentes tanto en la distribución como en la magnitud del potencial eléctrico sobre el cuero cabelludo. Esta influencia no es uniforme para todas las zonas corticales, sino que varía con la posición relativa de la fuente de actividad respecto a la grilla. Cuanto menor sea la distancia de la fuente al borde de la grilla mayor será la variación en la distribución del potencial eléctrico sobre el cuero cabelludo, pero menor será su atenuación. Justamente lo contrario sucede para fuentes cercanas al centro del sustrato, influenciando en mayor proporción a la magnitud de la señal medida en EEG. Dicha atenuación varía entre 2 y 13 veces para fuentes normales a la grilla y cercanas a ésta, reduciéndose notablemente para fuentes sobre las circunvoluciones (es decir, tangenciales a la grilla). Estos resultados coinciden con los de Zhang et al. (2006a), en donde fuentes dipolares dispuestas 
arbitrariamente y sin restricciones anatómicas fueron consideradas.

Respecto a la consideración de los huecos en el cráneo, se encontró que éstos sólo generan variaciones locales de segundo orden respecto a los del sustrato de la grilla, principalmente para fuentes tangenciales al cuero cabelludo. Los efectos encontrados coinciden con los reportados en la bibliografía (van den Broek et al., 1998; Benar y Gotman, 2002; Li et al., 2007), en donde se destaca el efecto local de los huecos y resalta su importancia para fuentes cercanas a éstos, principalmente orientadas tangencialmente. Dado que la incorporación de la grilla subdural conlleva a la deformación local de los tejidos blandos, alejando a la corteza de los huecos, los mismos no influyen en los cálculos posteriores y, por ende, su omisión en el modelo parece factible. Esta conclusión también encuentra soporte en el tamaño reducido de los huecos realizados (menos de $8 \mathrm{~mm}$ de radio) cuya influencia se torna despreciable (Li et al., 2007). Sin embargo, pueden existir casos en donde los huecos sean más grandes y/o queden más cercanos a la corteza, por lo que su consideración en el modelo debería ser evaluada.

Estos resultados permiten explicar en parte la variación del potencial eléctrico encontrado en diversos estudios de ECoG y EEG simultáneos. Distintos reportes destacan relaciones entre el máximo potencial eléctrico medido entre ECoG y EEG de entre 3 y 10 (Pacia y Ebersole, 1997; Tao et al., 2005, 2007a,b), y mayores aún (Hashiguchi et al., 2007). Las razones son generalmente atribuidas a la extensión de la zona epileptógena, el grado de sincronismo neuronal (Tao et al., 2007b; Hashiguchi et al., 2007) y la región anatómica en donde tiene lugar (Goldenholz et al., 2009). Sin embargo, los resultados aquí mostrados advierten de la necesidad de la consideración de la grilla subdural en estudios de EEG y ECoG simultáneos. Esto no concuerda con las hipótesis presentadas en Tao et al. (2007a,b), en donde se atribuyen efectos de la grilla menores a los del cráneo basados en la observación de la atenuación del ruido de fondo. En ellos se argumenta que los efectos de la grilla son similares tanto sobre la señal epiléptica como sobre el ruido de fondo, lo cual es parcialmente cierto dado que solo una proporción de las fuentes de fondo se encuentran en regiones corticales afectadas por la grilla. Menos aún coinciden con la hipótesis presentada en Tao et al. (2005), en donde se explicita la posibilidad de 
cancelación de efectos contrarios debidos a los huecos y la grilla. Luego, la ubicación de la zona epileptógena relativa a la grilla subdural es un parámetro de suma importancia a tener en consideración.

Menores efectos fueron hallados en el cálculo de la componente normal del campo magnético. Si bien altas variaciones de la distribución del campo debidas al modelo utilizado fueron encontradas para fuentes cercanas a la grilla y los huecos, la atenuación de la amplitud de señal no resultó ser de gran importancia. Se mostró que dichas influencias se deben a la variación de la corriente de volumen generadas por la grilla y los huecos, que puede generar diferencias normalizadas del $200 \%$ (principalmente para fuentes muy cercanas a la grilla). Estas diferencias repercuten en la solución del problema inverso (Schimpf et al., 2002), por lo que en caso de buscar resolverlo la consideración de un modelo con detalle es recomendable. Ejemplos de ello son los estudios cuya finalidad es evaluar la sensibilidad de MEG mediante la comparación directa entre las soluciones del problema inverso utilizando señales de MEG y de ECoG adquiridas simultáneamente. Si bien puede resultar innecesaria la adquisición de datos simultáneos (Agirre-Arrizubieta et al., 2009; Tanaka et al., 2010), son muchos los autores que confían en la supuesta idealidad de los mismos (Mikuni et al., 1997; Sutherling et al., 2001; Oishi et al., 2002; Shigeto et al., 2002), llegando algunos de ellos a realizar comparaciones de los resultados sobre modelos por demás simplistas (Mikuni et al., 1997; Oishi et al., 2002). Luego, el adecuado modelado de la cabeza permitiría reducir las fuentes de error al mínimo posible, tales como el ruido de fondo o la interferencia electromagnética propia de dichos estudios (Santiuste et al., 2008).

Sin embargo se vio que la variación del MAG promedio en el casco considerando fuentes en la ROI es muy pequeña, por lo que la atenuación de las señales de campo magnético debidas a la consideración de la grilla subdural es prácticamente despreciable. Luego, estudios en donde se busquen comparaciones de señales subdurales y de MEG mediante inspección visual o comparación directa de amplitudes no necesitarían la incorporación de un modelado detallado para la posterior interpretación de datos (al contrario que para señales de EEG). Dichos estudios engloban, entre otros, a aquellos que pretenden caracterizar el área de la zona epileptógena necesaria para 
su detectabilidad con MEG (Oishi et al., 2002; Shigeto et al., 2002; Santiuste et al., 2008).

Los modelos utilizados en este trabajo permitieron evaluar la influencia de los huecos en el cráneo y la grilla subdural en señales de ECoG. Si bien no implica variación en estudios cuya inspección visual es realizada, permite estudiar la necesidad de su incorporación en la resolución del problema inverso en ECoG, la cual no es usualmente tenida en cuenta (Korzyukov et al., 2007; Dümpelmann et al., 2009; Kim et al., 2010; Tanaka et al., 2010; Cho et al., 2011; Dümpelmann et al., 2011). Se mostró que la grilla subdural influye localmente en la determinación del potencial eléctrico en la corteza, llegando a ser influyente en fuentes cercanas a los electrodos. Asimismo, el efecto de los huecos resultó ser local y de segundo orden respecto a los de la grilla, llegando a ser similares a los de ésta en las vecindades de los mismos. Luego, la adecuada representación de la grilla y los huecos es recomendable en estudios que impliquen la estimación de fuentes puntuales en base a señales medidas con ECoG.

Finalmente se estudió la influencia del modelo en el cálculo de la SNR. Esto es de sumo interés en aplicaciones en donde la caracterización de la calidad de la señal es fundamental, como en la localización de fuentes epilépticas (Bast et al., 2006) y en aplicaciones de interfaz cerebro-computadora. La necesidad de SNR altas lleva a utilizar técnicas invasivas, las cuales mostraron ser de suma utilidad en estas aplicaciones (Miller et al., 2007; Shenoy et al., 2008). Sin embargo, sólo Ball et al. (2009) estudian la cuantificación de este parámetro utilizando señales simultáneas de EEG y ECoG.

En esta capítulo se calculó la ganancia en la SNR obtenida mediante métodos invasivos, y se la comparó tanto con métodos no invasivos simultáneos como también con métodos no invasivos prequirúrgicos. Se mostró que la ganancia en SNR de ECoG frente a señales de EEG simultáneas varía de 10 a 100 veces en la zona de interés, concordando con resultados experimentales existentes (Ball et al., 2009). Esta relación disminuye aproximadamente 10 veces si no se considera la grilla no conductora ni los huecos en el cráneo en las mediciones de EEG, indicando la influencia de la grilla subdural en la calidad de la señal sobre el cuero cabelludo y 
nuevamente contradiciendo la hipótesis presentada por Tao et al. (2005). Estos resultados permiten cuantificar la ganancia en la SNR esperable tras la incorporación de la grilla sobre la corteza, de modo que pueden resultar de interés en el análisis que lleve a la decisión de la realización de dichos estudios invasivos.

Diferentes fueron los resultados en el caso de considerar la medición de la componente normal del campo magnético, en donde la ganancia en SNR de ECoG frente a dichas señales de MEG simultáneas varía aproximadamente de 1 a 10 veces en la zona de interés. Esta relación prácticamente no se vio influenciada por la modificación del modelo, por lo que se evidencian ventajas de las señales de MEG frente a las de EEG. Estas ventajas radicarían en la detectabilidad de señales de interés por sobre el ruido incluso en presencia de la grilla subdural y huecos en el cráneo, las cuales permitirían realizar un análisis cualitativo en simultáneo al subdural más certero que si se utilizaran señales de EEG.

Cabe destacar que un modelo similar (Zhang et al., 2006a) fue realizado a fin de calcular la alteración de las señales de EEG debida a la incorporación de la grilla subdural. Si bien en éste se utiliza el MEF el mallado utilizado es más burdo, utilizando elementos 5 veces más grandes que los aquí utilizados, componiendo un modelo con número de elementos 10 veces menor. Como destacan los autores en dicho trabajo, esto impide el uso de fuentes restringidas anatómicamente, lo cual es solucionado en el presente estudio. Asimismo, en éste no son considerados los efectos simultáneos de la grilla y los huecos, por lo que se evalúa un modelo no del todo realista.

En el presente estudio se utilizaron fuentes dipolares restringidas anatómicamente como generadoras de señal. Si bien estos modelos son altamente aceptados en la comunidad científica (de Munck et al., 1988b; Merlet y Gotman, 1999), existen motivos para considerar su utilización no del todo conveniente en estudios de ECoG (Lachaux et al., 2003). Luego, modelos de fuentes distribuidas permitirán simular fuentes realistas, por lo que su uso (y desarrollo) formará parte de futuros estudios. Asimismo, deberían usarse modelos con mayor detalle de los electrodos, a fin de minimizar los efectos de la aproximación puntual de los mismos (Ollikainen et al., 2000). Sin embargo, un trabajo reciente (von Ellenrieder et al., 2012) 
mostró influencias despreciables para fuentes distantes a $1 \mathrm{~mm}$ utilizando electrodos profundos, por lo que se espera que su consideración no lleve a resultados muy diferentes de los aquí presentados. Próximos trabajos también focalizarán en la evaluación de diversas adaptaciones a la grilla utilizada con el fin de reducir sus efectos en la medición del potencial eléctrico sobre el cuero cabelludo, como pueden ser agujeros dispuestos en forma arbitraria entre los electrodos (Tao et al., 2007a). 


\section{Capítulo 5}

\section{Influencia de la incertidumbre en la posición de los electrodos}

\subsection{Introducción}

El problema inverso (PI) en EEG consiste en estimar las fuentes de actividad neuronal dadas las mediciones de potencial eléctrico en un número finito de puntos sobre el cuero cabelludo. Existen muchos factores que afectan la calidad de la solución de este problema, como el ruido electrónico de los sensores (Muravchik y Nehorai, 2001), la actividad cerebral de fondo (de Munck et al., 1992, 2002; Huizenga et al., 2002; Beltrachini et al., 2010) y los errores en el modelo de cabeza utilizado (Ollikainen et al., 1999; Wendel y Malmivuo, 2006; Wolters et al., 2006; von Ellenrieder et al., 2006). Asimismo, el problema de localización de fuentes requiere del conocimiento de la posición de los electrodos sobre el cuero cabelludo. Existen múltiples métodos (de Munck et al., 1991; Wang et al., 1996; Yoo et al., 1997; Khosla et al., 1999; von Ellenrieder et al., 2009a; de Munck et al., 2012) que son generalmente utilizados en la práctica con el fin de lograr minimizar dichos errores.

Los errores en la ubicación de los electrodos son producidos por la aleatoriedad del método utilizado a tal fin, así como por la técnica de registración empleada con objeto de ajustar y posicionar a dichos electrodos sobre la superficie del cuero cabelludo. Esta última fuente de error es inevitable ya que el cuero cabelludo y los 
tejidos blandos de la cabeza son ligeramente deformables, por lo que no existe un ajuste exacto entre la superficie del cuero cabelludo, generalmente obtenida a partir de imágenes de RM, y el conjunto de mediciones de la posición de los electrodos. En este capítulo se asume que la superficie del cuero cabelludo obtenida a partir de la RM se encuentra libre de errores, lo que es habitual en la práctica de EEG.

La influencia de los errores en el posicionamiento de los electrodos en la solución del PI ha sido estudiada anteriormente. En Khosla et al. (1999); Kavanagh et al. (1978); Towle et al. (1993); Hoey et al. (2000); Bruno et al. (2004) se adoptan modelos conductores esféricos, mientras que sólo en Wang y Gotman (2001) se considera un modelo realista de la cabeza. Asimismo, en la mayoría de estos trabajos se analiza el efecto de los errores en el posicionamiento de los electrodos considerando un método de resolución particular del PI. En este capítulo se presenta un método general basado en la cota de Cramér-Rao (CRB) (Kay, 1993) con el fin de analizar los efectos en el problema de localización considerando un modelo realista de la cabeza. Este método provee una cota inferior, ajustada, en la varianza de todo estimador insesgado de los parámetros de la fuente, por lo que constituye una generalización de los trabajos anteriores por ser éste independiente del algoritmo utilizado.

Con la finalidad de cuantificar el efecto de los errores en el posicionamiento de los electrodos se adopta un modelo aleatorio para la ubicación de los mismos. Esta aleatoriedad afecta la solución del problema directo (PD) mediante una aproximación lineal del potencial eléctrico en las inmediaciones de cada sensor. Luego, se calcula la sensibilidad del PD a estos parámetros y se obtiene la CRB. Los resultados muestran que el efecto de los errores en el posicionamiento de los electrodos es aproximadamente del mismo orden que la influencia del ruido electrónico de los sensores, en concordancia con resultados previos.

El presente capítulo se organiza del siguiente modo: en la Sección 5.2 se introduce el modelo aleatorio adoptado para realizar el análisis de los efectos de la incertidumbre en la posición de los electrodos. En la Sección 5.3 se obtiene la CRB para el presente problema. En la Sección 5.4 se discretiza el problema considerando el método de los elementos de contorno (BEM), el cual posibilita un tratamiento adecuado de la cota debido a resultados existentes relacionados con su implementa- 
ción. Esto permite calcular numéricamente la CRB, cuyos resultados se presentan en la Sección 5.5. Finalmente, la discusión de los resultados y las conclusiones del capítulo se presentan en la Sección 5.6.

\subsection{Descripción del modelo aleatorio}

En esta sección se describe el modelo aleatorio adoptado para representar al error en la determinación de la posición de los electrodos. Se consideran pequeñas perturbaciones de los electrodos respecto de su posición verdadera, cuyas fuentes recaen en la técnica de localización de los electrodos y en el proceso de ajuste de dichos sensores sobre la superficie del cuero cabelludo. Dado que existen múltiples causas diferentes para estos errores, resulta razonable la consideración de una distribución Gaussiana para las perturbaciones. Luego, variaciones en el potencial eléctrico medido con respecto a sus posiciones verdaderas aparecerán como variaciones en el potencial eléctrico medido en dichas posiciones verdaderas. Se encuentra la distribución probabilística del potencial eléctrico medido y se utiliza para la caracterización de la influencia del error en la posición de los electrodos en la solución del PI.

Sea $\Omega$ la superficie del cuero cabelludo sobre la cual se encuentran ubicados los electrodos. Sea $\boldsymbol{x}_{0}=\left\{\boldsymbol{x}_{i}^{0}\right\}_{i=1, \ldots, N s}$ el vector cuyos elementos representan a las posiciones de las coordenadas actuales (y desconocidas) de los $N_{s}$ electrodos, y $\boldsymbol{x}=\left\{\boldsymbol{x}_{i}\right\}_{i=1, \ldots, N s}$ la posición supuesta, en cualquier sistema de coordenadas. Para cada electrodo $\boldsymbol{x}_{i}$ posicionado en $\Omega$ se define un sistema de coordenadas locales, dado por $\left\{\boldsymbol{t}_{i}^{a}, \boldsymbol{t}_{i}^{b}, \boldsymbol{n}_{i}\right\}$, en donde $\boldsymbol{n}_{i}$ es el vector unitario normal a $\Omega$ en $\boldsymbol{x}_{i}$ y $\left\{\boldsymbol{t}_{i}^{a}, \boldsymbol{t}_{i}^{b}\right\}$ es la base ortonormal del plano $T_{X_{i}} \Omega$ tangente a $\Omega$ en $\boldsymbol{x}_{i}$ (Figura 5.1).

Si $\boldsymbol{x}_{i}^{0}$ es la posición verdadera del $i$-ésimo electrodo, es posible afirmar que $\boldsymbol{x}_{i}=\boldsymbol{x}_{i}^{0}+\boldsymbol{\xi}_{i}$, en donde $\boldsymbol{\xi}_{i}$ es una variable aleatoria que describe su desplazamiento, que es desconocido. Si se suponen pequeñas desviaciones respecto de $\boldsymbol{x}_{i}^{0}$, puede considerarse que $\boldsymbol{\xi}_{i}$ es un vector en $T_{X_{i}} \Omega$. Esto puede adoptarse dada la suposición de considerar a la reconstrucción de la superficie del cuero cabelludo sin errores y debido a que los electrodos se encuentran sobre dicha superficie y, por ende, la componente normal puede despreciarse por proyectar a los electrodos sobre la misma 


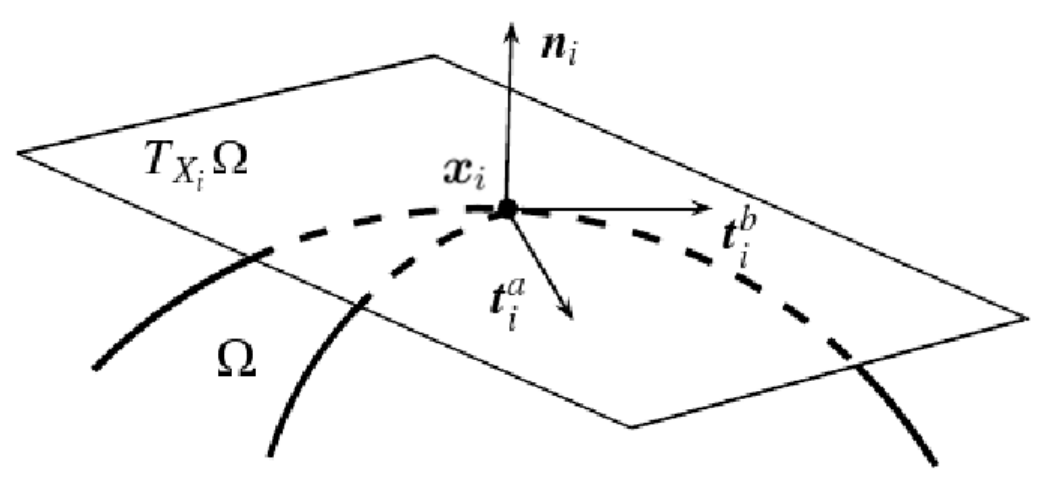

Figura 5.1: Representación del sistema de coordenadas locales correspondiente al i-ésimo electrodo.

superficie. Luego, se puede escribir $\boldsymbol{\xi}_{i}=\xi_{i}^{a} \boldsymbol{t}_{i}^{a}+\xi_{i}^{b} \boldsymbol{t}_{i}^{b}$. Se considera que $\xi_{i}^{a}$ y $\xi_{i}^{b}$ son variables aleatorias con distribución Gaussiana con media nula, i.e. $\mathbb{E}\left\{\boldsymbol{x}_{i}\right\}=\boldsymbol{x}_{i}^{0}$. La correlación entre la posición de dos electrodos diferentes permite modelar diferentes fuentes de error. De hecho, la medición de la posición de los electrodos tendrá errores descorrelacionados en el caso de utilizar una técnica de adquisición de la posición de un electrodo a la vez, tal como los lápices posicionadores electromagnéticos, o una baja correlación en caso de utilizar alguna otra técnica, e.g. de Munck et al. (1991); von Ellenrieder et al. (2009a); de Munck et al. (2012). Por otro lado, los errores debidos a un ajuste imperfecto entre los electrodos y la superficie del cuero cabelludo tienden a ser altamente correlacionados, ya que se relacionan con magnitudes globales, tal como rotaciones, traslaciones y escalado.

Sea $\varphi(\chi)$ la función escalar que representa al potencial eléctrico en algún punto $\chi$ de $\Omega$. Dado que la solución del PD en EEG es continua en $\Omega$, el cual se supone lo suficientemente suave respecto a $\boldsymbol{\chi}$, es posible escribir la expansión en serie de $\varphi\left(\boldsymbol{x}_{i}\right)$ alrededor de $\boldsymbol{x}_{i}^{0}$,

$$
\varphi\left(\boldsymbol{x}_{i}\right)=\varphi\left(\boldsymbol{x}_{i}^{0}\right)+\left.\nabla^{T} \varphi(\boldsymbol{\chi})\right|_{\boldsymbol{x}_{i}^{0}}\left(\boldsymbol{x}_{i}-\boldsymbol{x}_{i}^{0}\right)+\ldots
$$

Sea $\boldsymbol{\Phi}(\boldsymbol{x})=\left[\varphi\left(\boldsymbol{x}_{1}\right), \ldots, \varphi\left(\boldsymbol{x}_{N s}\right)\right]^{T}$ el vector de potenciales eléctricos medidos, i.e. el potencial eléctrico en cada una de las $N_{s}$ posiciones de los sensores. En este capítulo, 
al igual que en la totalidad de la tesis, se considera una referencia de potencial eléctrico promedio, i.e. se considera al promedio de todas las señales de potencial como referencia en la medición. Dado que el potencial eléctrico es una función de las posiciones de los electrodos, que a su vez son aleatorias, el vector $\boldsymbol{\Phi}(\boldsymbol{x})$ será también aleatorio. Utilizando definiciones y suposiciones previas, cualquier punto $\boldsymbol{x}_{i}$ puede ser caracterizado en el sistema de coordenadas locales por solo dos coordenadas distintas de cero, i.e. $\boldsymbol{x}_{i}=\left(x_{i}, y_{i}, 0\right)$. Luego, se puede escribir al vector de posiciones de los electrodos $\boldsymbol{x}$ como

$$
\boldsymbol{x}=\left[x_{1}, \ldots, x_{N_{s}}, y_{1}, \ldots, y_{N s}\right]^{T}
$$

y a la matriz de gradiente de potencial eléctrico en las verdaderas posiciones de los electrodos

$$
\boldsymbol{\nabla} \boldsymbol{\Phi}\left(\boldsymbol{x}_{0}\right)=\left[\begin{array}{ccc}
\left.\frac{\partial \varphi(\boldsymbol{\chi})}{\partial x_{1}}\right|_{\boldsymbol{x}_{1}^{0}} & \cdots & \left.\frac{\partial \varphi(\boldsymbol{\chi})}{\partial x_{N s}}\right|_{\boldsymbol{x}_{N s}^{0}} \\
\left.\frac{\partial \varphi(\boldsymbol{\chi})}{\partial y_{1}}\right|_{\boldsymbol{x}_{1}^{0}} & \cdots & \left.\frac{\partial \varphi(\boldsymbol{\chi})}{\partial y_{N s}}\right|_{\boldsymbol{x}_{N s}^{0}} ^{0}
\end{array}\right]^{T},
$$

en donde cada componente se encuentra en su correspondiente sistema de coordenadas locales. Luego, se puede encontrar una aproximación lineal de $\boldsymbol{\Phi}(\boldsymbol{x})$ alrededor de $\boldsymbol{x}_{0}$,

$$
\boldsymbol{\Phi}(\boldsymbol{x}) \approx \boldsymbol{\Phi}\left(\boldsymbol{x}_{0}\right)+\boldsymbol{E} \boldsymbol{D}\left(\boldsymbol{x}-\boldsymbol{x}_{0}\right)
$$

en donde $\boldsymbol{\Phi}\left(\boldsymbol{x}_{0}\right)=\left[\varphi\left(\boldsymbol{x}_{1}^{0}\right), \ldots, \varphi\left(\boldsymbol{x}_{N s}^{0}\right)\right]^{T}, \boldsymbol{E}=\left[\boldsymbol{I}_{N_{s}} \mid \boldsymbol{I}_{N_{s}}\right]$ y $\boldsymbol{D}=\operatorname{diag}\left\{\operatorname{vec}\left(\boldsymbol{\nabla} \boldsymbol{\Phi}\left(\boldsymbol{x}_{0}\right)\right)\right\}$. Debe notarse que $\boldsymbol{I}_{N}$ es la matriz identidad de $N \times N$ y, en el presente capítulo, diag $\mathrm{y}$ vec refieren a los operadores que retornan una matriz diagonal dado un vector $\mathrm{y}$ un vector dada una matriz, respectivamente.

Bajo estas suposiciones de aproximación lineal, y dado que $\boldsymbol{\xi}$ es Gaussiana, $\boldsymbol{\Phi}(\boldsymbol{x})$ será también un vector aleatorio con distribución Gaussiana, por lo que su caracterización resultará completa con los momentos de primer y segundo orden. La esperanza de las mediciones de potencial en (5.4) será $\mathbb{E}\{\boldsymbol{\Phi}(\boldsymbol{x})\}=\boldsymbol{\Phi}\left(\boldsymbol{x}_{0}\right)$, la solución del PD en las verdaderas posiciones de los electrodos. La matriz de covarianza del potencial eléctrico medido $\mathbb{C}_{\boldsymbol{\Phi}}$ vendrá dada por

$$
\begin{aligned}
\mathbb{C}_{\boldsymbol{\Phi}} & =\mathbb{E}\left\{(\boldsymbol{\Phi}(\boldsymbol{x})-\mathbb{E}\{\boldsymbol{\Phi}(\boldsymbol{x})\})(\boldsymbol{\Phi}(\boldsymbol{x})-\mathbb{E}\{\boldsymbol{\Phi}(\boldsymbol{x})\})^{T}\right\} \\
& =\mathbb{E}\left\{\boldsymbol{E} \boldsymbol{D}\left(\boldsymbol{x}-\boldsymbol{x}_{0}\right)\left(\boldsymbol{x}-\boldsymbol{x}_{0}\right)^{T} \boldsymbol{D}^{T} \boldsymbol{E}^{T}\right\}=\boldsymbol{E} \boldsymbol{D} \mathbb{C} \boldsymbol{x} \boldsymbol{D}^{T} \boldsymbol{E}^{T}
\end{aligned}
$$


en donde $\mathbb{C}_{\boldsymbol{x}}=\mathbb{E}\left\{\left(\boldsymbol{x}-\boldsymbol{x}_{0}\right)\left(\boldsymbol{x}-\boldsymbol{x}_{0}\right)^{T}\right\}$ es la matriz de covarianza del vector $\boldsymbol{x}$, y $\boldsymbol{E}$ y $\boldsymbol{D}$ son las definidas en (5.4). Luego, $\boldsymbol{\Phi}(\boldsymbol{x}) \sim \mathcal{N}\left(\boldsymbol{\Phi}\left(\boldsymbol{x}_{0}\right), \mathbb{C}_{\boldsymbol{\Phi}}\right)$, por lo que (5.5) indica que la relación entre la matriz de covarianza de las mediciones, $\mathbb{C}_{\boldsymbol{\Phi}}$, y la matriz de covarianza de la posición de los electrodos, $\mathbb{C}_{\boldsymbol{x}}$, dependerá solo del gradiente de potencial eléctrico.

Aquí se consideran dos modelos diferentes para la matriz de covarianza $\mathbb{C}_{\boldsymbol{x}}$, que permiten modelar diferentes fuentes de error. Se adopta un modelo descorrelacionado, i.e. $\mathbb{C}_{\boldsymbol{x}}=\sigma^{2} \boldsymbol{I}_{2 N_{s}}$, con el fin de representar a los errores en la medición de la posición de los sensores, y un modelo conjuntamente Gaussiano y altamente correlacionado, con matriz de covarianza $\mathbb{C}_{\boldsymbol{x}_{i j}}=\exp \left\{-\left\|\boldsymbol{x}_{i}-\boldsymbol{x}_{j}\right\|_{2}^{2} / 2 g^{2}\right\} \mathbf{t}_{i}^{\alpha_{i}} \cdot \mathbf{t}_{j}^{\alpha_{j}}, g=10 \mathrm{~cm}$, y $\alpha_{k}$ denota al superíndice $a$ si $k \leq N_{s}$ o al superíndice $b$ si $N s<k \leq 2 N_{s}$, con el fin de modelar errores que surjan del ajuste entre los electrodos y la superficie del cuero cabelludo. En las fórmulas anteriores, · representa al producto escalar.

\subsection{Cotas de desempeño}

El PI en EEG consiste en la estimación de los parámetros de la fuente basado en mediciones ruidosas del potencial eléctrico sobre el cuero cabelludo. Si bien existen múltiples métodos que resuelven este problema, la finalidad del presente análisis es estudiar en forma general la influencia de la incertidumbre en la posición de los electrodos en la estimación de los parámetros de la fuente. Esto se logra mediante la utilización de la CRB, la cual revela una cota inferior en la varianza de estimación de todos los estimadores insesgados, independientemente del algoritmo utilizado (Kay, 1993). Para métodos de estimación multiparamétrica, la CRB establece que

$$
\mathbb{E}\left\{(\boldsymbol{\theta}-\hat{\boldsymbol{\theta}})(\boldsymbol{\theta}-\hat{\boldsymbol{\theta}})^{T}\right\} \geq \mathbf{C R B}(\boldsymbol{\theta})=[\boldsymbol{J}(\boldsymbol{\theta})]^{-1}
$$

en donde $\hat{\boldsymbol{\theta}}$ es cualquier estimador insesgado del vector $\boldsymbol{\theta}$, CRB denota a la cota de Cramér-Rao, $\boldsymbol{J}(\boldsymbol{\theta})=\left\{J_{i j}\right\}_{i j}$ es la matriz de información de Fisher, y la desigualdad significa que la diferencia entre las matrices es positiva semi-definida. Para el caso particular de mediciones distribuidas en forma Gaussiana, la matriz de información 
de Fisher toma la forma (Kay, 1993)

$$
J_{i j}=\frac{\partial \boldsymbol{\Phi}\left(\boldsymbol{x}_{0}\right)^{T}}{\partial \theta_{i}} \mathbb{C}_{\boldsymbol{\Phi}}^{-1} \frac{\partial \boldsymbol{\Phi}\left(\boldsymbol{x}_{0}\right)}{\partial \theta_{j}}+\frac{1}{2} \operatorname{tr}\left\{\mathbb{C}_{\boldsymbol{\Phi}}^{-1} \frac{\partial \mathbb{C}_{\boldsymbol{\Phi}}}{\partial \theta_{i}} \mathbb{C}_{\boldsymbol{\Phi}}^{-1} \frac{\partial \mathbb{C}_{\boldsymbol{\Phi}}}{\partial \theta_{j}}\right\}
$$

en donde $\theta_{i}, i=1, \ldots, p$ son los parámetros de la fuente a estimar, i.e. elementos de $\boldsymbol{\theta}$. Nótese que el último término de (5.6) surge debido a que la matriz de covarianza $\mathbb{C}_{\boldsymbol{\Phi}}$ depende de los parámetros de la fuente.

Si se considera una única fuente dipolar estática (de Munck et al., 1988a), sólo será necesario estimar $p=6$ parámetros, tres de ellos correspondientes a la ubicación de la fuente y los restantes a la intensidad y orientación. Por simplicidad, se divide al cálculo de (5.6) en

$$
J_{i j}^{0}=\frac{\partial \boldsymbol{\Phi}\left(\boldsymbol{x}_{0}\right)^{T}}{\partial \theta_{i}} \mathbb{C}_{\boldsymbol{\Phi}}^{-1} \frac{\partial \boldsymbol{\Phi}\left(\boldsymbol{x}_{0}\right)}{\partial \theta_{j}}
$$

y

$$
J_{i j}^{1}=\frac{1}{2} \operatorname{tr}\left\{\mathbb{C}_{\boldsymbol{\Phi}}^{-1} \frac{\partial \mathbb{C}_{\boldsymbol{\Phi}}}{\partial \theta_{i}} \mathbb{C}_{\boldsymbol{\Phi}}^{-1} \frac{\partial \mathbb{C}_{\boldsymbol{\Phi}}}{\partial \theta_{j}}\right\}
$$

\subsection{Discretización}

En la presente sección se utiliza el modelo adoptado con el fin de calcular las variables requeridas para el cálculo de la cota. El método de elementos de contorno (BEM) (Geselowitz, 1967; Sarvas, 1987) es utilizado para discretizar a las ecuaciones correspondientes al PD y con el fin de calcular aproximaciones numéricas del potencial eléctrico, su gradiente y la sensibilidad de éstos respecto de los parámetros de la fuente. Con este método las superficies que definen al modelo de cabeza son discretizadas en un número grande de elementos triangulares, para llegar a la resolución de un sistema lineal que permite obtener una aproximación de los potenciales buscados en los vértices de los triángulos.

Para calcular el potencial eléctrico utilizando BEM se considera el uso de elementos que contemplen variación lineal del mismo sobre los elementos triangulares (de Munck, 1992). Luego, la solución del problema directo se encuentra dada por

$$
\boldsymbol{\Phi}(\mathbb{S})=\boldsymbol{G} \boldsymbol{\Phi}_{F},
$$


en donde $\boldsymbol{\Phi}(\mathbb{S})$ es el vector de $N$ elementos correspondiente al potencial eléctrico en los vértices de los nodos del mallado $\mathbb{S}$, y $\boldsymbol{G}$ es una matriz dependiente solo de la geometría y la conductividad eléctrica del modelo de cabeza. El término $\boldsymbol{\Phi}_{F}$ corresponde al potencial eléctrico generado por la misma fuente de actividad neuronal en un medio infinito y homogéneo y puede ser calculado en forma analítica. Dado que la matriz $\boldsymbol{G}$ no depende de los parámetros de la fuente, la sensibilidad del potencial eléctrico respecto de $\boldsymbol{\theta}$ se obtiene reemplazando $\boldsymbol{\Phi}_{F}$ por $\partial \boldsymbol{\Phi}_{F} / \partial \boldsymbol{\theta}$ en (5.9), el cual también se calcula analíticamente (Sarvas, 1987). La expresión correspondiente se da en el Apéndice C.1.

El potencial eléctrico en las $N_{s}$ posiciones de electrodos se obtiene mediante una interpolación lineal del valor de los vértices del elemento que contiene al electrodo (Muravchik y Nehorai, 2001). Si se define a la matriz rala de interpolación $\boldsymbol{H}_{l}(\boldsymbol{x})$ de dimensión $N_{s} \times N$, puede escribirse

$$
\Phi(\boldsymbol{x})=\boldsymbol{H}_{l}(\boldsymbol{x}) \Phi(\mathbb{S}) .
$$

Luego, se puede calcular (5.7) mediante

$$
\begin{aligned}
\boldsymbol{J}^{0} & =\frac{\partial \boldsymbol{\Phi}\left(\boldsymbol{x}_{0}\right)^{T}}{\partial \boldsymbol{\theta}} \mathbb{C}_{\boldsymbol{\Phi}}^{-1} \frac{\partial \boldsymbol{\Phi}\left(\boldsymbol{x}_{0}\right)}{\partial \boldsymbol{\theta}} \\
& =\frac{\partial \boldsymbol{\Phi}_{F}^{T}}{\partial \boldsymbol{\theta}} \boldsymbol{G}^{T} \boldsymbol{H}_{l}^{T}\left(\boldsymbol{x}_{0}\right) \mathbb{C}_{\boldsymbol{\Phi}}^{-1} \boldsymbol{H}_{l}\left(\boldsymbol{x}_{0}\right) \boldsymbol{G} \frac{\partial \boldsymbol{\Phi}_{F}}{\partial \boldsymbol{\theta}} .
\end{aligned}
$$

Para calcular el gradiente del potencial eléctrico en la posición de los electrodos se utiliza nuevamente BEM sobre el mismo mallado, pero ahora considerando un valor de potencial eléctrico constante sobre cada triángulo. Este gradiente es calculado en forma directa mediante la adaptación de la formulación presentada por von Ellenrieder et al. (2005a), tal como se explica en el Apéndice C.2. Esto conduce a

$$
\operatorname{vec}(\nabla \Phi(\mathbb{S}))=\boldsymbol{M f}
$$

en donde la matriz $\boldsymbol{M}$ solo depende de la geometría y de la conductividad eléctrica del modelo de cabeza. El vector $\boldsymbol{f}$ se relaciona al gradiente del potencial eléctrico generado por una fuente en un medio homogéneo e infinito. Expresiones analíticas de $\boldsymbol{f}$ y su sensibilidad respecto a parámetros de la fuente se dan en el Apéndice C.1. 
Nuevamente, se utiliza una matriz de interpolación rala $\boldsymbol{H}_{F}(\mathbb{X})$ con el fin de seleccionar al elemento de la discretización del mallado del cuero cabelludo en donde se encuentra cada electrodo. Luego, para calcular (5.8), se ve claramente de (5.5) que

$$
\frac{\partial \mathbb{C}_{\boldsymbol{\Phi}}}{\partial \theta_{j}}=\boldsymbol{E}\left(\frac{\partial \boldsymbol{D}}{\partial \theta_{j}} \mathbb{C}_{\boldsymbol{x}} \boldsymbol{D}+\boldsymbol{D} \mathbb{C}_{\boldsymbol{x}} \frac{\partial \boldsymbol{D}}{\partial \theta_{j}}\right) \boldsymbol{E}^{T}
$$

En el caso particular de considerar una matriz de covarianza diagonal (como se explica en la Sección 5.2) se muestra en el Apéndice C.3 que

$$
\frac{\partial \mathbb{C}_{\boldsymbol{\Phi}}}{\partial \theta_{j}}=2 \sigma^{2} \boldsymbol{E} \operatorname{diag}\left\{\left[\left(\boldsymbol{I}_{2} \otimes \boldsymbol{H}_{F}^{0}\right) \boldsymbol{M} \boldsymbol{f}\right] \circ\left[\left(\boldsymbol{I}_{2} \otimes \boldsymbol{H}_{F}^{0}\right) \boldsymbol{M} \frac{\partial \boldsymbol{f}}{\partial \theta_{j}}\right]\right\} \boldsymbol{E}^{T},
$$

en donde $\boldsymbol{H}_{F}^{0}=\boldsymbol{H}_{F}\left(\boldsymbol{x}_{0}\right), \otimes$ refiere al producto de Kronecker y $\circ$ refiere al producto de matrices elemento a elemento, también llamada multiplicación de Hadamard.

\subsection{Resultados}

En esta sección se utiliza la formulación previa para mostrar la influencia de los errores en la posición de los electrodos en la solución del PI en EEG. Para eso se consideró un modelo de cabeza realista conformado por tres capas, con superficies entre capas discretizadas en 2562 vértices (5120 elementos triangulares), disponible en SPM8 ${ }^{1}$ (Friston et al., 2007). Esta discretización implica triángulos de $6 \mathrm{~mm}$ de lado promedio, lo cual es suficiente para considerar a las superficies como suaves. La Figura 5.2 muestra dicha discretización del cuero cabelludo. Las tres capas consideradas corresponden al cerebro, cráneo y cuero cabelludo, cuyas conductividades eléctricas se asumieron $0,3 \mathrm{~S} / \mathrm{m}, 0,02 \mathrm{~S} / \mathrm{m}$ y $0,3 \mathrm{~S} / \mathrm{m}$, respectivamente (Oostendorp et al., 2000; Wendel y Malmivuo, 2006; Zhang et al., 2006b). Se consideraron 120 electrodos posicionados de acuerdo a una extensión del estándar 10-20 (Deuschl y Eisen, 2000) (Figura 5.2). El problema directo se resolvió utilizando BEM, tal como se describió en la sección anterior, utilizando el enfoque de cráneo aislado (Meijs et al., 1989).

\footnotetext{
${ }^{1}$ Disponible en http://www.fil.ion.ucl.ac.uk/spm/
} 


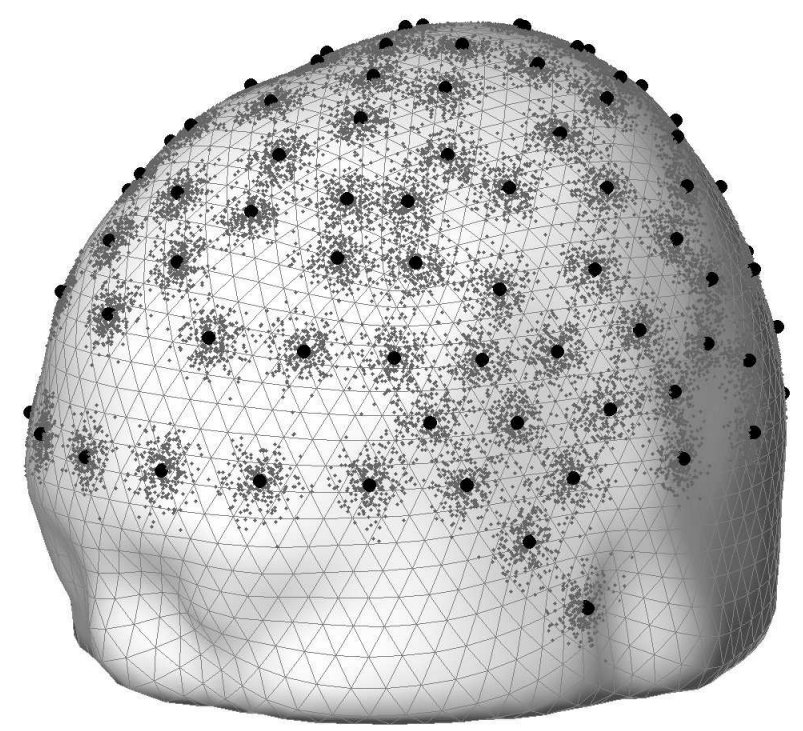

Figura 5.2: Posicionamiento de los electrodos sobre el modelo realista de cabeza adoptado. Con puntos negros se muestra el valor medio de la posición de los electrodos, de acuerdo a una extensión del estándar 10-20. Con puntos grises se muestran 250 realizaciones diferentes de las posiciones de los electrodos, tal como se explicó en la Sección 2. Se muestra también la discretización considerada.

Se calculó la CRB de acuerdo a la expresión (5.6) asumiendo independencia entre las posiciones de los electrodos. Dado que la cota para una única fuente dipolar se encuentra representada por una matriz de $6 \times 6$ que resulta difícil de visualizar, se estudian dos factores escalares relacionados a la posición de la fuente. El primero de ellos es el volumen del elipsoide de 90 \% de probabilidad (Hochwald y Nehorai, 1997; Muravchik y Nehorai, 2001). Este factor es un intervalo de confianza tridimensional que permite cuantificar al volumen del elipsoide que contiene a la fuente con un $90 \%$ de probabilidad, considerando distribuciones conjuntamente Gaussianas. Asimismo, se consideró el cálculo de la cota en la desviación estándar de la profundidad de la fuente, la cual se encuentra dada por la raíz cuadrada del elemento de la matriz de la cota correspondiente a la profundidad de la fuente.

En las Figuras 5.3 y 5.4 se muestra, sobre un corte coronal, el volumen del elipsoide de $90 \%$ de probabilidad y la cota de la desviación estándar en la estimación de la profundidad de la fuente considerando fuentes dipolares orientadas tangen- 


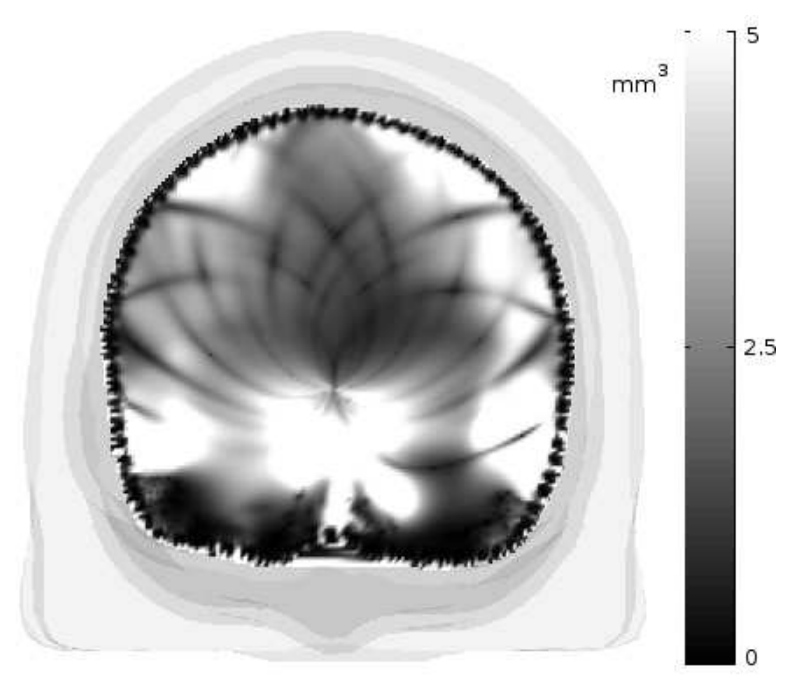

Figura 5.3: Volumen del elipsoide de confianza de $90 \%$ en la estimación de la posición de las fuentes. Los resultados corresponden a fuentes orientadas tangencialmente ubicadas sobre un corte coronal del cerebro. El nivel de gris indica el mínimo volumen del elipsoide de confianza de $90 \%$ de probabilidad considerando cualquier estimador insesgado de la posición de la fuente.

cialmente. Cada punto del corte representa a la posición de la fuente dipolar, y el diferente tono de gris es proporcional al volumen (Figura 5.3) o a la desviación estándar (Figura 5.4).

Se consideraron errores en el posicionamiento de los electrodos con una desviación estándar de $5 \mathrm{~mm}$ para cada electrodo, una hipótesis pesimista obtenida a partir de datos de electrodos posicionados manualmente (Wang y Gotman, 2001). Se adoptaron a fuentes dipolares de $20 \mathrm{nAm}$. Debe notarse que en las figuras se muestran las mallas correspondientes al cuero cabelludo y cráneo solo por claridad de las mismas (el tono de gris en ellas no tiene el significado anteriormente descripto). Las figuras muestran que las regiones con mayor influencia debida a los errores en la posición de los electrodos son la corteza cerebral (la capa exterior) y el cuerpo calloso (materia blanca profunda).

En la Figura 5.5 se comparan los efectos en el problema inverso de EEG debidos al error en la posición de los electrodos con aquellos originados por el ruido electrónico en los sensores. El modelo adoptado para éste último es Gaussiano de media nula 


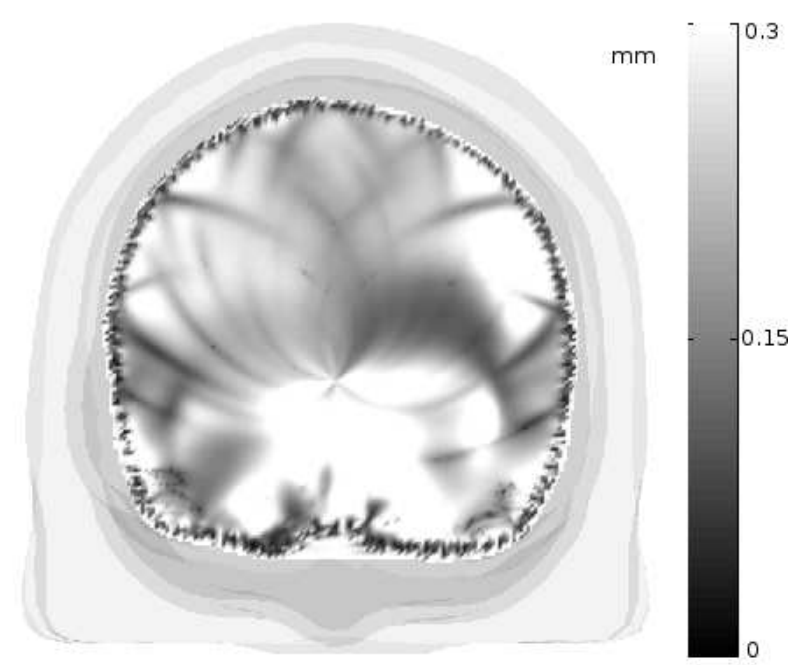

Figura 5.4: Raíz cuadrada de la CRB en la estimación de la profundidad de la fuente. Los resultados corresponden a fuentes orientadas tangencialmente ubicadas sobre un corte coronal del cerebro. El nivel de gris indica la mínima desviación estándar en la estimación de la profundidad de la fuente considerando cualquier estimador insesgado de su posición.

y desviación estándar $0,5 \mu \mathrm{V}$ (Muravchik y Nehorai, 2001), descorrelacionado entre sensores. Si bien existen otras fuentes de error, tal como la actividad cerebral de fondo y los errores en el modelo, se consideró al error electrónico de fondo debido a su naturaleza inevitable. En la Figura 5.5 se muestra el cociente entre las cotas en la estimación de la profundidad de la fuente en la presencia de errores en la posición de los electrodos y en presencia de ruido electrónico en función de la posición de la fuente. Puede verse que la influencia de los errores en la posición de los electrodos es relevante solo si se trata de fuentes en la cercanía de la corteza cerebral, en donde su influencia es a lo sumo igual a la debida al ruido electrónico en los sensores.

Algunos puntos en común deben notarse de las Figuras 5.3-5.5. En principio, pueden observarse líneas curvas oscuras que cruzan los cortes, las cuales denotan zonas de menor valor de la cota. Éstas se encuentran asociadas a regiones con menor magnitud del gradiente de potencial eléctrico, como se muestra en von Ellenrieder y Muravchik (2000). Asimismo, se vislumbran imprecisiones en los resultados correspondientes a fuentes muy cercanas a la superficie interior del cráneo, representado 


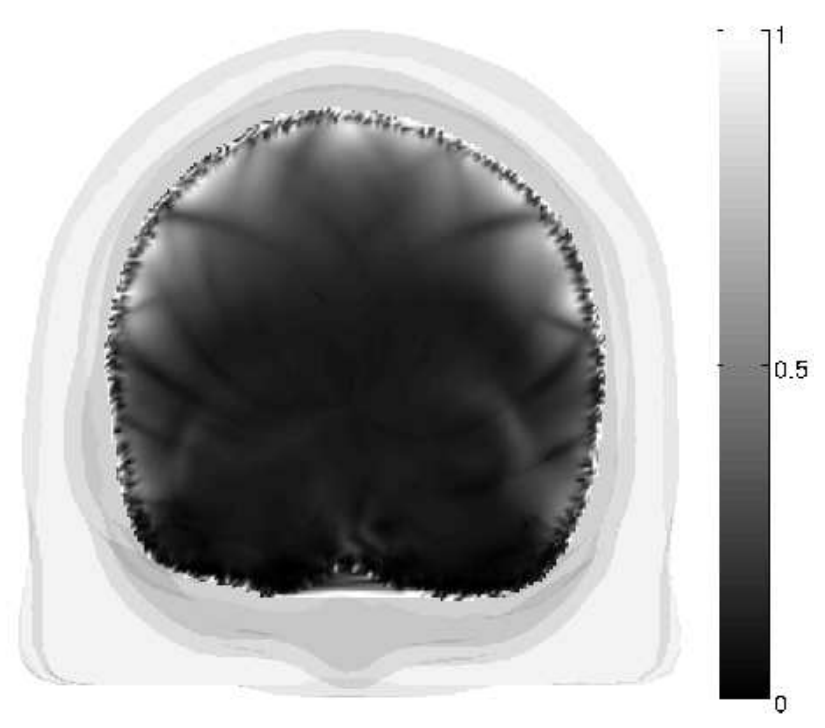

Figura 5.5: Relación entre las incertidumbres en la solución del problema inverso debido al efecto del error en la posición de los electrodos y debido al ruido electrónico. Este cociente se calcula entre las raíces cuadradas de la CRB en la estimación de la profundidad de la fuente, para cada posición considerada.

en las figuras mediante una región de resultados sumamente variables. La razón se encuentra en los errores numéricos que el BEM comete para fuentes con profundidad menor a la mitad del lado de los elementos triangulares de los elementos del mallado de la superficie (Haueisen et al., 1997).

Tal como se mostró en la Sección 5.2, se consideraron dos fuentes de error posibles: el error en el posicionamiento de los electrodos modelado como independiente entre sensores y el de registración entre los electrodos y la superficie del cuero cabelludo, modelado como espacialmente correlacionado. En la Figura 5.6 se muestra la comparación entre los resultados para ambos casos. Esta figura muestra la cota inferior en la desviación estándar de estimadores insesgados de la profundidad de la fuente en función de la desviación estándar considerada en la posición de los electrodos. Las fuentes fueros separadas en dos grupos, uno de ellos representando dipolos en la materia gris sobre una capa de $11 \mathrm{~mm}$ de espesor por debajo de la superficie interior del cráneo, y la otra más profunda representando fuentes ubicadas en la materia blanca. Si bien es sabido que las fuentes de mayor importancia en señales 


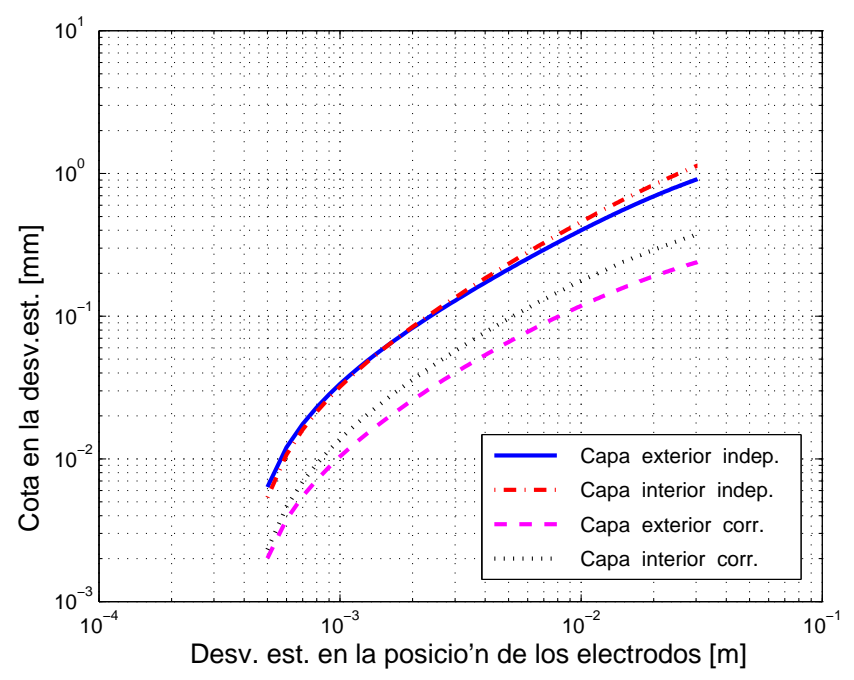

Figura 5.6: Raíz cuadrada de la CRB en la estimación de la profundidad de la fuente en función de la desviación estándar en la posición de los electrodos. Los resultados corresponden a los efectos promedio de las perturbaciones en las capas interior y exterior considerando errores en la posición de los electrodos correlacionados e independientes.

de EEG son las neuronas piramidales ubicadas en la corteza de la materia gris, se simularon fuentes que pueden representar materia gris profunda en cerebros atípicos. Los resultados mostrados corresponden al promedio de más de 200 fuentes en cada capa. La comparación entre estos resultados y la cota inferior en la estimación de la profundidad debida al ruido electrónico de fondo se muestra en la Figura 5.7. Puede verse que el efecto de los errores en la posición de los electrodos es mayor para fuentes corticales y errores no correlacionados. Sin embargo, hasta en el peor de los casos la desviación estándar en la estimación de la profundidad de la fuente puede ser tan pequeña como 1mm, como puede observarse en la Figura 5.6.

Si bien se muestran resultados sólo considerando fuentes orientadas tangencialmente, el efecto es ligeramente menor en caso de considerar fuentes orientadas en forma radial. Esto resulta razonable dado que la distribución de potencial eléctrico debido a fuentes orientadas radialmente presenta menor amplitud que la de las fuentes orientadas tangencialmente. También debe notarse que el método aquí propuesto puede ser utilizado para calcular el efecto de la incertidumbre en la posición de los 


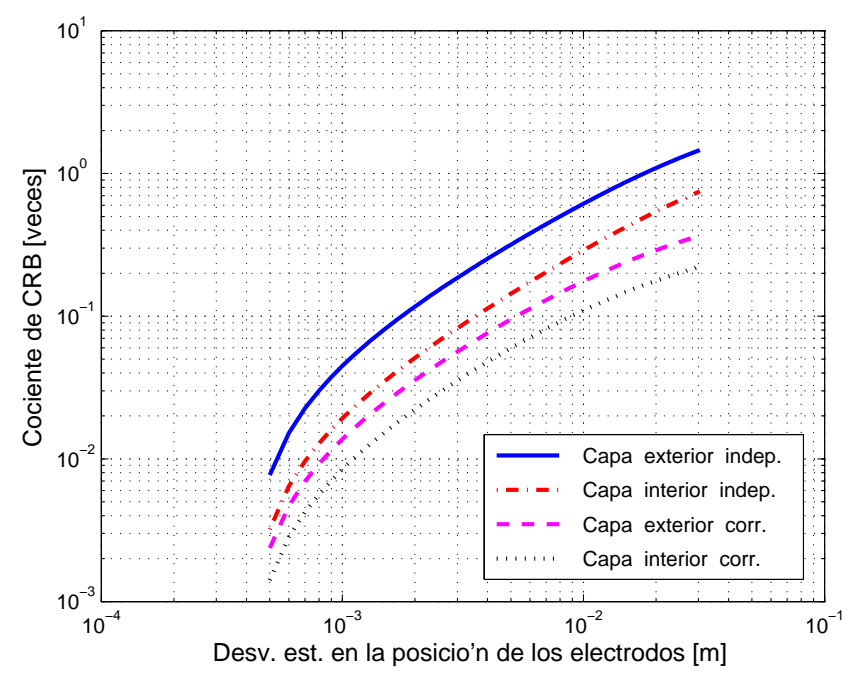

Figura 5.7: Variaciones del cociente entre la raíz cuadrada de la CRB en a estimación de la profundidad de la fuente debido a errores en la ubicación de los electrodos y al ruido electrónico en función de la desviación estándar en la posición de los electrodos. Los resultados corresponden a los efectos promedio de las perturbaciones en las capas interior y exterior considerando errores en la posición de los electrodos correlacionados e independientes.

electrodos considerando otra clase de fuentes de actividad cerebral, tal como fuentes distribuidas sobre una línea (Yetik et al., 2005) o un parche (Yetik et al., 2006). Esto repercute solamente en el vector $\boldsymbol{f}$ en la ecuación (5.12). Se presupone que los resultados se mantendrán para fuentes modeladas por un conjunto de dipolos.

Tal como se explicó anteriormente, los resultados mostrados corresponden a una configuración de 120 electrodos sobre el cuero cabelludo. Un análisis similar fue realizado considerando 64 electrodos, conduciendo prácticamente a los mismos resultados. La relación entre las incertidumbres en la solución del PI debido a los efectos de la posición de los electrodos y al ruido electrónico resultó ser menor, lo cual es razonable dado que la desviación estándar debida al ruido en los sensores aumenta a medida que el número de electrodos decrece. 


\subsection{Conclusiones}

Con el fin de cuantificar el efecto de los errores de posición de los electrodos en la localización, se modelaron como variables aleatorias y, a través de una aproximación lineal del potencial eléctrico alrededor de los electrodos, se determinó la influencia de esta aleatoriedad en la resolución del PD. Luego, considerando a esta aleatoriedad como ruido se calculó la CRB de la varianza de los parámetros de la fuente. Los resultados aquí presentados corresponden a un análisis general en el desempeño óptimo de los estimadores, sin proponer ningún método que consiga dichos resultados, si bien algunos que parecen hacerlo ya fueron presentados en trabajos previos (Khosla et al., 1999; Wang y Gotman, 2001).

Se concluyó en que el efecto de los errores en la medición de los electrodos y en la registración entre dichas mediciones y la superficie del cuero cabelludo no son de suma importancia en el uso regular de EEG. En particular se mostró que para fuentes dipolares y desviación estándar de $5 \mathrm{~mm}$ en la ubicación de los electrodos los errores en la estimación de la ubicación de la fuente son despreciables en comparación con la desviación estándar de los estimadores debidas al ruido electrónico en la adquisición de EEG. La máxima influencia debida a la incertidumbre en el posicionamiento de los electrodos se encontró para fuentes orientadas tangencialmente. Sin embargo, aún en el peor de los casos la cota en la desviación estándar de la estimación de la posición de la fuente no es mayor a $1 \mathrm{~mm}$, por lo que puede ser despreciado en caso de considerar otras fuentes de error, tal como la actividad de fondo (de Munck et al., 2002; Huizenga et al., 2002; Beltrachini et al., 2010) o variaciones respecto del modelo de cabeza de tres capas homogéneas (Ollikainen et al., 1999; Wendel y Malmivuo, 2006; Wolters et al., 2006; Vatta et al., 2010). Los resultados también muestran que el uso de una gorra con electrodos, la cual puede introducir errores correlacionados en la determinación de su posición, no implica mayores efectos en la precisión de la estimación de la ubicación de la fuente que de considerar errores descorrelacionados de electrodos colocados individualmente.

Finalmente debe destacarse que los resultados encontrados son consistentes con 
reportes previos en donde se utilizan resolvedores del PI particulares (Khosla et al., 1999; Wang y Gotman, 2001), lo cual implica que los errores inevitables en la registración de los electrodos y la superficie del cuero cabelludo y de la posición de éstos mismos per se son tolerables en la solución del PI en EEG. Más aún, el efecto de los errores en el posicionamiento de los electrodos es lo suficientemente pequeño para sugerir que una técnica tan precisa en la medición de dichas posiciones no es necesaria. Sin embargo, en el caso de considerar todas las fuentes de error en forma conjunta puede resultar de interés el conocimiento preciso de la posición con la finalidad de reducir al mínimo una de estas causas. 


\section{Capítulo 6}

\section{Estimación por constricción de la matriz de covarianza}

\subsection{Introducción}

La actividad cerebral de fondo de EEG está correlacionada espacial y temporalmente. La caracterización de dicha actividad de fondo es de sumo interés, pues permite estudiar mapas de coherencia y de correlación (ten Caat et al., 2008; Liu et al., 2010) entre diferentes bandas de frecuencias (Freeman, 2005), así como redes estructurales y funcionales del cerebro (Bullmore y Sporns, 2009). Otros usos de importancia radican en la localización de fuentes de actividad cerebral, ya que la adecuada caracterización espacio-temporal de la actividad de fondo es necesaria para la obtención de resultados fidedignos (Lütkenhöner, 1998). Al respecto se ha mostrado que la incorporación de esta información mejora los resultados de la localización, reduciendo la varianza de los parámetros a estimar (de Munck et al., 2002).

Una posible y adecuada caracterización de la actividad cerebral de fondo consiste en suponer que es un proceso aleatorio y especificar su momento de segundo orden, i.e. la matriz de covarianza. En caso de suponer que la amplitud del ruido de fondo viene dada por un proceso Gaussiano de media nula, la matriz de covarianza caracteriza completamente a dicho proceso. Sin embargo, la matriz de covarianza 
general del proceso espacio-temporal de actividad de fondo es muy grande. Con la finalidad de reducir su dimensionalidad es generalmente descompuesta como el producto de Kronecker de dos matrices de covarianza de menor dimensión, llamadas matrices de covarianza espacial y temporal (de Munck et al., 2002; Huizenga et al., 2002; Bijma et al., 2005). Para estimar estas matrices en forma adecuada se necesitan múltiples realizaciones del proceso bajo análisis. En estudios relacionados con mediciones de EEG, tales como en potenciales evocados y epilepsia, el valor medio de la señal medida es usualmente la señal de interés. Las matrices de covarianza generalmente se estiman utilizando técnicas muestrales (empíricas) o de máxima verosimilitud (de Munck et al., 2002). Sin embargo, si el número de realizaciones disponibles es pequeño en comparación al número de entradas espacio-temporales de las mediciones estos estimadores presentarán un pobre desempeño, conllevando a problemas del tipo numérico y estadístico (Stein, 1956; Khatri y Rao, 1987). Esta situación sucede, por ejemplo, en algunos estudios ligados a epilepsia, en donde el número de realizaciones se reduce al de espigas interictales observadas y medidas que muestran una misma morfología y distribución espacial, las cuales generalmente no son más de diez. Para mejorar este desempeño es que en el presente capítulo se plantea el uso de los estimadores de constricción.

La metodología de constricción para la estimación de las matrices de covarianza ha sido objeto de estudio en diferentes áreas del conocimiento, tales como el análisis multivariable (Sancetta, 2008), economía (Ledoit y Wolf, 2001), biología (Schäfer y Strimmer, 2005), y procesamiento de señales (Stoica et al., 2008; Chen et al., 2010). La propuesta de constricción consiste en realizar un balance entre el sesgo y la (co)varianza del estimador (James y Stein, 1961), con el fin de reducir la distancia entre el estimador propuesto (que es sesgado) y la matriz de covarianza verdadera. Este estimador se construye mediante una combinación lineal (generalmente convexa) de dos estimadores diferentes: uno con menor (co)varianza que sesgo y otra con menor sesgo que (co)varianza. Luego, la estimación se completa mediante la búsqueda del coeficiente de la combinación que minimiza la distancia entre el estimador y la covarianza verdadera. Asimismo, se busca que el estimador buscado sea tanto numéricamente estable así como estadísticamente consistente. En el presen- 
te capítulo se presentan condiciones suficientes para su consistencia y un resultado sobre la estabilidad numérica de los estimadores propuestos. Asimismo, se utilizan modelos existentes de las matrices de covarianza temporal (Bijma et al., 2003) y espacial (de Munck et al., 1992) de la actividad cerebral de fondo y se muestra como la utilización de esta información permite obtener estimadores aún mejores, considerando datos sintéticos y reales.

Con el objeto de evaluar el desempeño de los estimadores de constricción se compararon tanto con otros estimadores comúnmente utilizados, así como con el mejor estimador insesgado que podría obtenerse, cuyo desempeño está dado por la cota de Cramér-Rao (CRB). Se muestra que el estimador presentado siempre es preferible frente al estimador muestral y, bajo algunas hipótesis es incluso mejor que cualquier estimador insesgado de las matrices de covarianza de EEG.

El presente capítulo se organiza del siguiente modo: en la Sección 6.2 se formula el problema de la estimación de la matriz de covarianza espacio-temporal y se muestra como los estimadores comúnmente utilizados pueden fallar cuando el número de realizaciones disponibles es pequeño. Para paliar este hecho, en la Sección 6.3 se presenta el método de estimación por constricción de la matriz de covarianza, del cual se muestran resultados teóricos que promueven su utilización. Asimismo, se presentan cotas de desempeño que permiten su comparación con estimadores insesgados. En la Sección 6.4 se muestran resultados utilizando datos sintéticos y reales, los cuales permiten validar el entorno de estimación aquí propuesto. Finalmente, en la Sección 6.5 se presentan conclusiones del capítulo y líneas de trabajo futuro.

Notación: En el presente capítulo se utiliza Diag $\{\cdot\}$ para referir al operador que, dada una matriz cuadrada, devuelve una matriz de las mismas dimensiones, con los mismos elementos sobre la diagonal y ceros fuera de la misma; y $\operatorname{diag}\{\cdot\}$ al operador que, dada una matriz cuadrada, devuelve un vector columna cuyos elementos son los de la diagonal de la matriz de entrada. 


\subsection{Estimadores usuales}

En esta sección se presenta el modelo de señal usualmente adoptado en EEG, junto a varios de los estimadores de la matriz de covarianza más frecuentemente utilizados. Se muestran algunos problemas asociados a estos estimadores cuando el número de realizaciones es pequeño. Luego, se presentan cotas de desempeño para estimadores insesgados de la matriz de covarianza, los cuales sirven como referencia en la comparación presentada en la Sección 6.3.

\subsubsection{Modelo de señal}

Con la finalidad de establecer el modelo de señal es preciso realizar algunas suposiciones iniciales. Se considera que las diferentes realizaciones medidas resultan de la misma fuente neuronal, contaminadas éstas con ruido correlacionado espaciotemporalmente, tal como en de Munck et al. (2002). Las mediciones de EEG se encuentran también contaminadas con ruido electrónico de los sensores. Sin embargo, dado que esta fuente de ruido se encuentra bien caracterizada y es considerablemente de menor amplitud que la actividad de fondo (Mosher et al., 1993; Radich y Buckley, 1995; Muravchik y Nehorai, 2001) su estimación puede ser desestimada o absorbida en la estimación de la actividad de fondo.

Sea $P$ el número de sensores ubicados en la gorra de adquisición, $N$ el número de muestras temporales de cada realización y $K$ la cantidad de realizaciones disponibles. Asimismo, sea $\boldsymbol{Y}$ la matriz aleatoria de $P \times N$ correspondiente al fenómeno bajo análisis, e $\boldsymbol{Y}^{k}$ la $k$-ésima realización medida. Se considera una distribución Gaussiana matricial para las mediciones (de Munck et al., 2002; Kollo y von Rosen, 2005), independiente en las realizaciones, con media $\boldsymbol{M}$ asociada al término de señal, covarianza temporal $\boldsymbol{\Sigma}$ y covarianza espacial $\boldsymbol{\Omega}$, i.e. $\boldsymbol{Y} \sim \mathcal{N}_{P \times N}(\boldsymbol{M}, \boldsymbol{\Sigma}, \boldsymbol{\Omega})$, en donde se escoge $\boldsymbol{\Omega}=\mathbb{E}\left\{(\boldsymbol{Y}-\boldsymbol{M})(\boldsymbol{Y}-\boldsymbol{M})^{T}\right\}$ y $\boldsymbol{\Sigma}=\mathbb{E}\left\{(\boldsymbol{Y}-\boldsymbol{M})^{T}(\boldsymbol{Y}-\boldsymbol{M})\right\} / c$, en donde $c$ es una constante que permite asegurar la adecuada normalización de potencia (Kollo y von Rosen, 2005). Bajo estas hipótesis, el problema consiste en encontrar estimadores adecuados de $\boldsymbol{\Sigma}$ y $\boldsymbol{\Omega}$. 


\subsubsection{Covarianza muestral y de máxima verosimilitud}

Los estimadores más utilizados en la bibliografía son los estimadores muestrales. Dadas $K$ realizaciones, los estimadores (sesgados) de las matrices de covarianza temporal y espacial vendrán dados por

$$
\begin{aligned}
& \widehat{\boldsymbol{\Sigma}}_{K}=\frac{1}{P K} \sum_{k=1}^{K}\left(\boldsymbol{Y}^{k}-\overline{\boldsymbol{Y}}\right)^{T}\left(\boldsymbol{Y}^{k}-\overline{\boldsymbol{Y}}\right) \\
& \widehat{\boldsymbol{\Omega}}_{K}=\frac{1}{N K} \sum_{k=1}^{K}\left(\boldsymbol{Y}^{k}-\overline{\boldsymbol{Y}}\right)\left(\boldsymbol{Y}^{k}-\overline{\boldsymbol{Y}}\right)^{T},
\end{aligned}
$$

en donde $\overline{\boldsymbol{Y}}=1 / K \sum_{k=1}^{K} \boldsymbol{Y}^{k}$ es el estimador muestral de la media. Puede probarse fácilmente que el rango de $\widehat{\Omega}_{K}$ es menor o igual a $K \operatorname{mín}(P, N)$. Luego, resulta evidente verificar que $\widehat{\Omega}_{K}$ presenta problemas numéricos si $K \operatorname{mín}(P, N) \leq P$. Si se asume una situación típica de $P=120$ y $N=20$, resulta que $\widehat{\Omega}_{K}$ tendrá problemas si $K \leq 6$, lo cual es una situación usual en algunos estudios relacionados a EEG, tal como epilepsia. Luego, en estos casos el estimador muestral de la covarianza espacial no resulta ser adecuado. Asimismo, puede mostrarse (ver Sección 6.3) que si se considera un número pequeño de realizaciones ambos estimadores muestrales de las matrices de covarianza presentan un pobre desempeño.

Otra opción consiste en encontrar el estimador de máxima verosimilitud (MLE), como se propone en de Munck et al. (2002). Los MLE de las covarianzas temporal y espacial vendrán dadas por

$$
\begin{aligned}
& \widehat{\boldsymbol{\Sigma}}_{M L}=\frac{1}{P K} \sum_{k=1}^{K}\left(\boldsymbol{Y}^{k}-\overline{\boldsymbol{Y}}\right)^{T} \widehat{\boldsymbol{\Omega}}_{M L}^{-1}\left(\boldsymbol{Y}^{k}-\overline{\boldsymbol{Y}}\right), \\
& \widehat{\boldsymbol{\Omega}}_{M L}=\frac{1}{N K} \sum_{k=1}^{K}\left(\boldsymbol{Y}^{k}-\overline{\boldsymbol{Y}}\right) \widehat{\boldsymbol{\Sigma}}_{M L}^{-1}\left(\boldsymbol{Y}^{k}-\overline{\boldsymbol{Y}}\right)^{T} .
\end{aligned}
$$

Si bien estos estimadores resultan ser más adecuados que los muestrales, se muestra fácilmente que (6.4) presenta problemas en las mismas situaciones que $\widehat{\Omega}_{K}$, y luego $\widehat{\Omega}_{M L}$ no será numéricamente estable para algunos estudios de epilepsia. Más aún, la estimación de los MLE se encuentra definida por un sistema de ecuaciones matriciales acoplado. Como se detalla en Bijma et al. (2003), los estimadores derivados de (6.3) 
y (6.4) requieren de un gran costo computacional, lo cual conforma una razón válida para evitar su utilización.

Luego, el problema abordado en el presente capítulo es el siguiente: considerando un número pequeño de realizaciones, encontrar estimadores de las matrices de covarianza temporal y espacial numéricamente estables, que requieran de un bajo costo computacional y que se encuentren lo más "cerca" posible a las verdaderas (y desconocidas) matrices de covarianza. Para ello se proponen los estimadores de constricción.

\subsection{Estimadores de constricción}

El método de estimación de matrices de covarianza por constricción consiste en buscar un estimador sesgado que minimice la distancia (bajo alguna métrica) entre el estimador propuesto y la verdadera matriz de covarianza. En este capítulo se considera a la norma Frobenius de la diferencia entre matrices como función de distancia. Si bien no es la única métrica posible (Smith, 2005; Peeters et al., 2009), es razonable para los objetivos presentes, permitiendo derivar expresiones de sencilla implementación.

Sea $\Omega$ la matriz de covarianza espacial a estimar. El método de estimación por constricción permite buscar un estimador estructurado $\widehat{\Omega}_{S}$ de la matriz de covarianza verdadera (y desconocida) $\Omega$ de modo que el error cuadrático medio (ECM), definido por $\operatorname{ECM}\left(\widehat{\boldsymbol{\Omega}}_{S}\right)=\mathbb{E}\left\{\left\|\widehat{\boldsymbol{\Omega}}_{S}-\boldsymbol{\Omega}\right\|_{F}^{2}\right\}$, sea mínimo. Con el objeto de minimizar el ECM, Ledoit y Wolf (2001) proponen un estimador basado en la combinación lineal convexa de dos estimadores; el primero, $\boldsymbol{T}_{\Omega}$, con relativamente baja varianza y alto sesgo, y el segundo, $\boldsymbol{S}_{\Omega}$, con menor sesgo y mayor varianza que $\boldsymbol{T}_{\Omega}$. Luego, el estimador de constricción queda definido por

$$
\widehat{\boldsymbol{\Omega}}_{S}=\alpha_{\Omega} \boldsymbol{T}_{\Omega}+\left(1-\alpha_{\Omega}\right) \boldsymbol{S}_{\Omega}
$$

en donde el coeficiente $\alpha_{\Omega} \in[0,1]$ se llama coeficiente de constricción espacial. Si bien no es la única combinación posible (Li et al., 2008; Zhu et al., 2008), esta elección permite obtener el parámetro de forma sencilla y con bajo costo computacional. 
Utilizando resultados existentes (Schäfer y Strimmer, 2005), se encuentra que el parámetro de constricción con el que se obtiene el mínimo ECM viene dado por

$$
\alpha_{\Omega}^{o}=\frac{\mathbb{V}\left\{\boldsymbol{S}_{\Omega}\right\}-\mathbb{C}\left\{\boldsymbol{S}_{\Omega}, \boldsymbol{T}_{\Omega}\right\}+\operatorname{tr}\left\{\mathbb{E}\left\{\boldsymbol{S}_{\Omega}-\boldsymbol{T}_{\Omega}\right\} \mathbb{B}\left(\boldsymbol{S}_{\Omega}\right)\right\}}{\mathbb{E}\left\{\left\|\boldsymbol{S}_{\Omega}-\boldsymbol{T}_{\Omega}\right\|_{F}^{2}\right\}},
$$

en donde $\mathbb{V}\left\{\boldsymbol{S}_{\Omega}\right\}=\mathbb{E}\left\{\left\|\boldsymbol{S}_{\Omega}-\mathbb{E}\{\boldsymbol{\Omega}\}\right\|_{F}^{2}\right\}$ es la varianza de $\boldsymbol{S}_{\Omega}$ considerando la norma Frobenius, $\mathbb{C}\left(\boldsymbol{S}_{\Omega}, \boldsymbol{T}_{\Omega}\right)=\operatorname{tr}\left\{\mathbb{E}\left\{\left(\boldsymbol{T}_{\Omega}-\mathbb{E}\left\{\boldsymbol{T}_{\Omega}\right\}\right)\left(\boldsymbol{S}_{\Omega}-\mathbb{E}\left\{\boldsymbol{S}_{\Omega}\right\}\right)^{T}\right\}\right\}$ es la covarianza entre $\boldsymbol{S}_{\Omega}$ y $\boldsymbol{T}_{\Omega}$ considerando la misma norma, y $\mathbb{B}\left(\boldsymbol{S}_{\Omega}\right)=\mathbb{E}\left\{\boldsymbol{S}_{\Omega}\right\}-\boldsymbol{\Omega}$ es la matriz de sesgo de $\boldsymbol{S}_{\Omega}$. Una expresión simplificada se obtiene en caso de escoger $\boldsymbol{S}_{\Omega}$ como un estimador insesgado de $\boldsymbol{\Omega}$ (i.e. $\mathbb{B}\left(\boldsymbol{S}_{\Omega}\right)=\mathbf{0}_{P}$, la matriz nula de $P \times P$ ), llevando a

$$
\alpha_{\Omega}^{o}=\frac{\mathbb{V}\left\{\boldsymbol{S}_{\Omega}\right\}-\mathbb{C}\left\{\boldsymbol{S}_{\Omega}, \boldsymbol{T}_{\Omega}\right\}}{\mathbb{E}\left\{\left\|\boldsymbol{S}_{\Omega}-\boldsymbol{T}_{\Omega}\right\|_{F}^{2}\right\}} .
$$

A partir de aquí, y por simplicidad, se considera que $\boldsymbol{S}_{\Omega}$ es el estimador muestral insesgado de la matriz de covarianza $\boldsymbol{\Omega}$, i.e. $\boldsymbol{S}_{\Omega}=K /(K-1) \widehat{\boldsymbol{\Omega}}_{K}$.

El mismo procedimiento se lleva a cabo para encontrar al estimador de constricción de $\boldsymbol{\Sigma}$. Considerando $\boldsymbol{T}_{\Sigma}$ y $\boldsymbol{S}_{\Sigma}$ como los estimadores sesgado y muestral, respectivamente, el parámetro de constricción óptimo $\alpha_{\Sigma}^{o}$ se encuentra mediante el simple reemplazo de $\boldsymbol{\Omega}$ por $\boldsymbol{\Sigma}$ en (6.6) ó (6.7).

A continuación se presentan resultados teóricos acerca de algunas propiedades de los estimadores propuestos, que permiten la adecuada elección de la matriz de sesgo. Las demostraciones se encuentran en el Apéndice D.

Teorema 6.3.1 Sean $\widehat{\Sigma}_{S}$ y $\widehat{\Omega}_{S}$ los estimadores de constricción definidos por (6.5) y (6.7). Luego, las relaciones

$$
\begin{aligned}
& \operatorname{ECM}\left(\widehat{\boldsymbol{\Sigma}}_{S}\right) \leq \operatorname{ECM}\left(\boldsymbol{S}_{\Sigma}\right), \\
& \operatorname{ECM}\left(\widehat{\boldsymbol{\Omega}}_{S}\right) \leq \operatorname{ECM}\left(\boldsymbol{S}_{\Omega}\right),
\end{aligned}
$$

se verifican siempre. Más aún, si existe una constante $K_{1}$ independiente de $K$ tal que $\mathbb{E}\left\{\left(Y_{p n}^{k}-M_{p n}\right)^{2}\right\} \leq K_{1}$ para todo $p=1, \ldots, P$ y $n=1, \ldots, N$, luego $\widehat{\Sigma}_{S}$ y $\widehat{\Omega}_{S}$ son consistentes bajo la norma Frobenius.

El Teorema 6.3.1 establece dos importantes resultados concernientes a los estimadores de constricción. En principio, determina que el ECM del estimador propuesto 
es siempre menor o igual que el ECM de la matriz de covarianza muestral. En segundo lugar, establece como condición suficiente del estimador de constricción para ser consistente que el segundo momento central de las mediciones sea finito. Dado que en el presente trabajo se asume una distribución Gaussiana para las mediciones, la condición se cumple siempre, por lo que los estimadores propuestos son consistentes bajo la norma Frobenius.

Sin embargo, el Teorema 6.3.1 no aclara nada sobre la estabilidad numérica de los estimadores. Por ello se muestra que un estimador sesgado $\boldsymbol{T}$ con número de condición adecuado puede ayudar en este sentido.

Teorema 6.3.2 Sea $\lambda_{i}(\boldsymbol{C})$ el $i$-ésimo autovalor de la matriz $\boldsymbol{C}$ de modo que $\lambda_{1}(\boldsymbol{C})=$ $\lambda_{m}(\boldsymbol{C})$ es el menor, $\lambda_{M}(\boldsymbol{C})$ es el mayor y se encuentran ordenados en orden ascendente. Luego, los autovalores de $\widehat{\Sigma}_{S} y \widehat{\Omega}_{S}$ se encuentran acotados por

$$
\begin{gathered}
\alpha_{\Sigma} \lambda_{n}\left(\boldsymbol{T}_{\Sigma}\right)+\left(1-\alpha_{\Sigma}\right) \lambda_{m}\left(\boldsymbol{S}_{\Sigma}\right) \leq \lambda_{n}\left(\widehat{\boldsymbol{\Sigma}}_{S}\right) \leq \alpha_{\Sigma} \lambda_{n}\left(\boldsymbol{T}_{\Sigma}\right)+\left(1-\alpha_{\Sigma}\right) \lambda_{M}\left(\boldsymbol{S}_{\Sigma}\right), \\
\alpha_{\Omega} \lambda_{p}\left(\boldsymbol{T}_{\Omega}\right)+\left(1-\alpha_{\Omega}\right) \lambda_{m}\left(\boldsymbol{S}_{\Omega}\right) \leq \lambda_{p}\left(\widehat{\boldsymbol{\Omega}}_{S}\right) \leq \alpha_{\Omega} \lambda_{p}\left(\boldsymbol{T}_{\Omega}\right)+\left(1-\alpha_{\Omega}\right) \lambda_{M}\left(\boldsymbol{S}_{\Omega}\right),
\end{gathered}
$$

$\operatorname{para} n=1, \ldots, N$ y $p=1, \ldots, P$.

Luego, con el objeto de obtener estimadores de constricción numéricamente aptos, la matriz de sesgo $\boldsymbol{T}$ debe ser bien condicionada, de acuerdo al Teorema 6.3.2. Asimismo, y de ser posible, ésta debe presentar una estructura similar a la de la verdadera matriz de covarianza, pues del Teorema 6.3.1 se ve que a medida que el parámetro de constricción se acerca a la unidad, el estimador homónimo es mejor que el muestral. Dado que $\mathbb{V}\{\boldsymbol{S}\}$ es dependiente de los datos (y no puede cambiarse), se deben escoger $\mathbb{C}(\boldsymbol{S}, \boldsymbol{T})$ y $\mathbb{E}\left\{\|\boldsymbol{T}-\boldsymbol{S}\|_{F}^{2}\right\}$ lo más pequeños posibles. Esto puede obtenerse reduciendo la aleatoriedad de $\boldsymbol{T}$ y, como resulta evidente, escogerlo de modo que sea lo más parecido posible a la matriz de covarianza verdadera.

\subsubsection{Selección de la estructura subyacente}

Con el objeto de obtener estimadores de constricción de buen desempeño, y haciendo uso de los Teoremas 6.3.1 y 6.3.2, se proponen diferentes alternativas para 
las matrices de sesgo $\boldsymbol{T}_{\Omega}$ y $\boldsymbol{T}_{\Sigma}$, basados en modelos existentes de las covarianzas de la actividad de fondo.

Múltiples modelos han sido propuestos como estructura subyacente de la matriz de covarianza espacial de la actividad de fondo (de Munck et al., 1992, 1997; Huizenga et al., 2002). En el presente se considerará el modelo propuesto en de Munck et al. (1992), debido a su fundamento fisiológico y su simplicidad. El modelo descripto en dicho trabajo consiste en una capa de fuentes dipolares aleatorias que describen a la actividad cerebral de fondo, considerando un modelo de cabeza esférica de tres capas. En el caso de asumir fuentes dipolares orientadas radialmente y uniformemente distribuidas en una superficie esférica de radio $r_{0}$, la covarianza espacial entre el $i$-ésimo y el j-ésimo electrodo resulta ser proporcional a

$$
\sum_{n=1}^{\infty}(2 n+1) r_{0}^{2 n} P_{n}\left(\cos \left(\beta_{i j}\right)\right)=\left(1-r_{0}^{4}\right)\left(1-2 \cos \left(\beta_{i j}\right) r_{0}^{2}+r_{0}^{4}\right)^{-3 / 2}-1
$$

en donde $\beta_{i j}$ es el ángulo entre el $i$-ésimo y el j-ésimo electrodo con respecto al centro de la esfera, y $P_{n}(x)$ en el polinomio de Legendre de grado $n$. De más está decir que la matriz de covarianza verdadera no responderá en forma exacta a este modelo, pero bien puede capturar una gran porción de la estructura subyacente de dicha matriz. Luego, esta matriz es utilizada para construir al estimador sesgado $\boldsymbol{T}_{\Omega}$.

Sea $\boldsymbol{D}_{\Omega}$ la matriz cuyos elementos se encuentran dados por la expresión (6.12) normalizada, i.e. con coeficiente de correlación entre el $i$-ésimo y el j-ésimo electrodo definido por

$$
D_{i j}^{\Omega}=\frac{\left(1-r_{0}^{4}\right)\left(1-2 \cos \left(\beta_{i j}\right) r_{0}^{2}+r_{0}^{4}\right)^{-3 / 2}-1}{r_{0}^{2}\left(3-r_{0}\right)\left(1-r_{0}^{2}\right)^{-1}} .
$$

Para posiciones de electrodos conocidas, $\boldsymbol{D}_{\Omega}$ dependerá sólo de la elección de $r_{0}$. Basado en (6.13) se proponen dos estructuras para $\boldsymbol{T}_{\Omega}$. La primera se encuentra basada en la suposición de igual potencia en los sensores debida a la actividad cerebral correlacionada espacialmente. Esto lleva a la elección

$$
\boldsymbol{T}_{\Omega}=\left(\operatorname{tr}\left\{\boldsymbol{S}_{\Omega}\right\} / P\right) \boldsymbol{D}_{\Omega}
$$

en donde $\operatorname{tr}\left\{\boldsymbol{S}_{\Omega}\right\} / P$ es la potencia media en los electrodos. Esta estructura puede resultar no del todo realista, pues existe evidencia de que la actividad de fondo no se 
encuentra igualmente distribuida en la superficie del cuero cabelludo, por lo que la potencia entre los electrodos debida a actividad espacialmente correlacionada puede variar de un sensor a otro (de Jongh et al., 2001; Liu et al., 2010). Si se considera esto se llega a la segunda estructura aquí propuesta,

$$
\boldsymbol{T}_{\Omega}=\operatorname{Diag}\left\{\boldsymbol{S}_{\Omega}\right\}^{1 / 2} \boldsymbol{D}_{\Omega} \operatorname{Diag}\left\{\boldsymbol{S}_{\Omega}\right\}^{1 / 2},
$$

en donde la pre y post multiplicación por $\operatorname{Diag}\left\{\boldsymbol{S}_{\Omega}\right\}^{1 / 2}$ asegura la normalización de potencia adecuada.

Un análisis similar se puede llevar adelante con la matriz de covarianza temporal. Si bien existen algunos modelos que permiten su descripción con detalle (Bijma et al., 2003), no se encontraron modelos tan simples como en el caso espacial. Por esta razón se propuso una estructura sencilla cuyos elementos normalizados se encuentran dados por

$$
e^{-\kappa \Delta t_{i j}} \cos \left(\omega_{f} \Delta t_{i j}\right)
$$

en donde $\Delta t_{i j}$ es la diferencia temporal entre la $i$-ésima y la $j$-ésima muestras temporales, y $\kappa \mathrm{y} \omega_{f}$ son variables a determinar. Como se explica en Bijma et al. (2003), $\omega_{f}$ se relaciona con la frecuencia dominante de la actividad cerebral de fondo, la cual es generalmente una combinación entre los ritmos Alfa $(\sim 10 \mathrm{~Hz})$, Zeta $(\sim 6 \mathrm{~Hz})$ y Delta $(\sim 4 \mathrm{~Hz})$.

Nuevamente, esta estructura es utilizada a fin de construir el estimador sesgado $\boldsymbol{T}_{\Sigma}$. Sea $\boldsymbol{D}_{\Sigma}$ la matriz con elementos $D_{i j}^{\Sigma}$ dados por (6.14). En el caso de suponer estacionareidad, el estimador propuesto será

$$
\boldsymbol{T}_{\Sigma}=\left(\operatorname{tr}\left\{\boldsymbol{S}_{\Sigma}\right\} / N\right) \boldsymbol{D}_{\Sigma}
$$

en donde $\operatorname{tr}\left\{\boldsymbol{S}_{\Sigma}\right\} / N$ es la potencia media a lo largo de las muestras temporales. Al igual que en el caso espacial, esta hipótesis no es del todo realista, por lo que se propone el esquema

$$
\boldsymbol{T}_{\Sigma}=\operatorname{Diag}\left\{\boldsymbol{S}_{\Sigma}\right\}^{1 / 2} \boldsymbol{D}_{\Sigma} \operatorname{Diag}\left\{\boldsymbol{S}_{\Sigma}\right\}^{1 / 2}
$$

en donde la variación de potencia en el tiempo se encuentra contemplada. Los esquemas propuestos en esta sección se resumen en el Cuadro 6.1 con el nombre de esquemas 5 y 6 . 
Cuadro 6.1: Esquemas considerados en el trabajo.

\begin{tabular}{|c|c|c|}
\hline$N^{o}$ & Estructura espacial $\left(\boldsymbol{T}_{\Omega}\right)$ & Estructura temporal $\left(\boldsymbol{T}_{\Sigma}\right)$ \\
\hline \hline 1 & $\mathbf{I}_{P}$ & $\mathbf{I}_{N}$ \\
\hline 2 & $\left(\operatorname{tr}\left\{\boldsymbol{S}_{\Omega}\right\} / P\right) \mathbf{I}_{P}$ & $\left(\operatorname{tr}\left\{\boldsymbol{S}_{\Sigma}\right\} / N\right) \mathbf{I}_{N}$ \\
\hline 3 & $\operatorname{Diag}\left(\boldsymbol{S}_{\Omega}\right)$ & $\operatorname{Diag}\left(\boldsymbol{S}_{\Sigma}\right)$ \\
\hline 4 & $\left(\operatorname{diag}\left(\boldsymbol{S}_{\Omega}\right) \operatorname{diag}\left(\boldsymbol{S}_{\Omega}\right)^{T}\right)^{1 / 2}$ & $\left(\operatorname{diag}\left(\boldsymbol{S}_{\Sigma}\right) \operatorname{diag}\left(\boldsymbol{S}_{\Sigma}\right)^{T}\right)^{1 / 2}$ \\
\hline 5 & $\left(\operatorname{tr}\left\{\boldsymbol{S}_{\Omega}\right\} / P\right) \boldsymbol{D}_{\Omega}$ & $\left(\operatorname{tr}\left\{\boldsymbol{S}_{\Sigma}\right\} / N\right) \boldsymbol{D}_{\Sigma}$ \\
\hline 6 & $\operatorname{Diag}\left(\boldsymbol{S}_{\Omega}\right)^{1 / 2} \boldsymbol{D}_{\Omega} \operatorname{Diag}\left(\boldsymbol{S}_{\Omega}\right)^{1 / 2}$ & $\operatorname{Diag}\left\{\boldsymbol{S}_{\Sigma}\right\}^{1 / 2} \boldsymbol{D}_{\Sigma} \operatorname{Diag}\left\{\boldsymbol{S}_{\Sigma}\right\}^{1 / 2}$ \\
\hline
\end{tabular}

\subsubsection{Esquemas propuestos e implementación numérica}

Se pretende comparar el desempeño de los estimadores de constricción utilizando los esquemas propuestos con otros esquemas genéricos utilizados en la bibliografía. Se contemplan estimadores muestrales para calcular $\alpha^{o}$ (Schäfer y Strimmer, 2005), para lo cual se necesita estimar $\mathbb{V}\{\boldsymbol{S}\}$ y $\mathbb{C}\{\boldsymbol{S}, \boldsymbol{T}\}$ de las mediciones. Sean las matrices $\boldsymbol{W}^{k}$ con elementos $w_{i j}^{k}=\left(Y_{i}^{k}-\overline{Y_{i}}\right)\left(Y_{j}^{k}-\overline{Y_{j}}\right)^{T}$ y $\overline{\boldsymbol{W}}=1 / K \sum_{k=1}^{K} \boldsymbol{W}^{k}$, en donde $Y_{i}^{k}$ refiere a la $i$-ésima fila de $\boldsymbol{Y}^{k}$. Luego, puede mostrarse (Schäfer y Strimmer, 2005) que la varianza en el caso espacial viene dada por

$$
\widehat{\mathbb{V}\{\boldsymbol{S}\}}=\frac{K}{N^{2}(K-1)^{3}} \sum_{k=1}^{K}\left\|\boldsymbol{W}^{k}-\overline{\boldsymbol{W}}\right\|_{F}^{2} .
$$

Del mismo modo se obtiene una expresión para el caso temporal. Con el fin de estimar $\mathbb{C}\{\boldsymbol{S}, \boldsymbol{T}\}$ debe escogerse una matriz $\boldsymbol{T}$. En el Cuadro 6.1 se enumeran los cinco modelos considerados en el trabajo.

El primero consiste en tomar $\boldsymbol{T}=\boldsymbol{I}$, en donde $\boldsymbol{I}$ es la matriz identidad. Ésta es la estructura más trivial, dado que no utiliza información del proceso bajo estudio. Es generalmente utilizada en regresión regularizada y métodos de regularización de Tikhonov (Hastie et al., 2001). El segundo es el esquema propuesto por Ledoit y Wolf (2001), en donde $\boldsymbol{T}=(\operatorname{tr}\{\boldsymbol{S}\} / r) \boldsymbol{I}$, con $r=N$ ó $r=P$ según se trate de la estimación de la matriz de covarianza temporal o espacial, respectivamente. Éste corresponde a una extensión del modelo diagonal en donde se considera un vec- 
tor de pesos uniforme. La característica en común de ambos es la consideración de igual varianza para cada parámetro, reduciendo a todos los coeficientes desconocidos en sólo uno. Para salvar esta limitación, Schäfer y Strimmer (2005) proponen un esquema diagonal con elementos no necesariamente iguales, listado en el cuadro como modelo 3. Sin embargo, el modelo diagonal puede resultar poco adecuado para múltiples problemas. Por esta razón los mismos autores proponen un modelo de matriz llena, en donde $\boldsymbol{T}=\left(\operatorname{diag}(\boldsymbol{S}) \operatorname{diag}(\boldsymbol{S})^{T}\right)^{1 / 2}$. Si bien esta estructura (estructura 4) admite elementos distintos de cero fuera de la diagonal, presenta un problema crítico (así como la estructura 3), que es su directa dependencia con la matriz de covarianza muestral. Como se mostró anteriormente, este estimador presenta problemas numéricos cuando el número de realizaciones es lo suficientemente pequeño, lo que puede llevar a las estructuras 3 y 4 a ser mal condicionadas (de acuerdo al Teorema 6.3.2). Los modelos de estructura presentados en la sub-sección anterior se muestran también en el cuadro, llamados estructuras 5 y 6 . Para cada una de ellas se evalúa $\mathbb{C}\{\boldsymbol{S}, \boldsymbol{T}\}$. Su cálculo resulta evidente de las definiciones previas, así como el cómputo de $\widehat{\mathbb{V}\{\boldsymbol{S}\}}$.

Cabe destacar que las estructuras subyacentes 5 y 6 dependen de los valores $r_{0}$ (para el caso espacial) y $\omega_{f}$ y $\kappa$ (para el caso temporal). En este trabajo se estiman estas variables de las realizaciones utilizando un algoritmo (de muy baja complejidad computacional) de región de confianza reflectivo (trust-region-reflective), basado en Coleman y Li (1994, 1996).

\subsubsection{Cotas de desempeño}

Tal como se explicó en la Sección 6.1, el objetivo principal de los estimadores de constricción es minimizar el ECM del estimador de la matriz de covarianza. Como consecuencia de ello se espera mejorar el desempeño del estimador respecto a los insesgados. Con la finalidad de calcular el desempeño óptimo de un estimador insesgado se considera el cálculo de la CRB, la que devuelve una cota inferior en la varianza del estimador, sin importar el algoritmo utilizado. Para estimación multi- 
paramétrica, la CRB establece

$$
\mathbb{E}\left\{(\boldsymbol{\gamma}-\widehat{\gamma})(\boldsymbol{\gamma}-\widehat{\gamma})^{T}\right\} \geq \operatorname{CRB}(\boldsymbol{\gamma})=[\boldsymbol{J}(\boldsymbol{\gamma})]^{-1},
$$

en donde $\widehat{\boldsymbol{\gamma}}$ es un estimador insesgado de $\boldsymbol{\gamma}, \mathbf{C R B}$ es la cota de Cramér-Rao, $\boldsymbol{J}(\boldsymbol{\gamma})$ es la matriz de información de Fisher, y la desigualdad implica que la diferencia entre las matrices es positiva definida. Para el caso particular de considerar mediciones distribuidas en forma Gaussiana con media nula y matriz de covarianza $\Upsilon$, la matriz de información de Fisher estará dada por (Kay, 1993)

$$
J_{i j}=\frac{1}{2} \operatorname{tr}\left\{\boldsymbol{\Upsilon}^{-1} \frac{\partial \Upsilon}{\partial \gamma_{i}} \boldsymbol{\Upsilon}^{-1} \frac{\partial \Upsilon}{\partial \gamma_{j}}\right\}
$$

en donde $\gamma_{i}, i=1, \ldots, p$ son los parámetros a estimar, i.e. los elementos de $\gamma$.

En el presente caso los parámetros de interés serán los elementos de las matrices de covarianza $\boldsymbol{\Sigma}$ y $\boldsymbol{\Omega}$. Sea vech $(\cdot)$ el operador que apila las columnas de una matriz cuadrada una debajo de la otra en una única columna, pero empezando desde el elemento de la diagonal. Dado que toda matriz de covarianza es hermítica, se nota claramente que vech $(\cdot)$ contiene el máximo número de parámetros diferentes que la matriz de covarianza puede tener. Sea $\gamma=\left[\boldsymbol{\gamma}_{\sigma}^{T}, \boldsymbol{\gamma}_{\omega}^{T}\right]^{T}$, en donde $\boldsymbol{\gamma}_{\sigma}=\operatorname{vech}(\boldsymbol{\Sigma}) \mathrm{y}$ $\gamma_{\omega}=\operatorname{vech}(\boldsymbol{\Omega})$. Luego, los elementos de $\gamma$ serán todos los parámetros desconocidos de interés. Se muestra en el Apéndice D.3 que la CRB en el ECM de cualquier estimador insesgado de $\boldsymbol{\Sigma}$ y $\boldsymbol{\Omega}$ están dadas por

$$
\begin{gathered}
E C M(\widehat{\boldsymbol{\Sigma}}) \geq \boldsymbol{\rho}_{\sigma}^{T} \operatorname{diag}\left\{\left(\boldsymbol{C}-\boldsymbol{B} \boldsymbol{A}^{-1} \boldsymbol{B}^{T}\right)^{-1}\right\}, \\
\operatorname{ECM}(\widehat{\boldsymbol{\Omega}}) \geq \boldsymbol{\rho}_{\omega}^{T} \operatorname{diag}\left\{\left(\boldsymbol{A}-\boldsymbol{B}^{T} \boldsymbol{C}^{-1} \boldsymbol{B}\right)^{-1}\right\},
\end{gathered}
$$

en donde las matrices $\boldsymbol{A}, \boldsymbol{B}$ y $\boldsymbol{C}$ y los vectores $\boldsymbol{\rho}_{\sigma}$ y $\boldsymbol{\rho}_{\omega}$ sólo dependen de los elementos de $\boldsymbol{\Sigma}$ y $\boldsymbol{\Omega}$, y sus definiciones son dadas en el mismo apéndice. Debe notarse que el caso aquí presentado es una extensión de los resultados mostrados en Dogandžić y Nehorai (2000), en donde se asume independencia temporal.

\subsection{Resultados}

En esta sección se presentan resultados en dos modos diferentes. En primer lugar se simulan señales contaminadas con ruido correlacionado espacial y temporalmente 
utilizando un modelo de cabeza real y se muestra como los estimadores de constricción mejoran el desempeño en la estimación de las matrices de covarianza. Luego, se evalúa la técnica propuesta considerando los diferentes esquemas utilizando datos de EEG tomados de un paciente epiléptico.

\subsubsection{Resultados utilizando datos sintéticos}

En este estudio de simulación se evalúan los estimadores de constricción utilizando datos sintéticos. En principio se generaron señales de EEG contaminadas con ruido electrónico y actividad de fondo resultante de fuentes dispuestas aleatoriamente. Luego, considerando diferentes cantidades de realizaciones disponibles se estimaron las matrices de covarianza utilizando el método propuesto y se compararon los resultados tanto con los estimadores muestrales como con la CRB para estimadores insesgados desarrollada en la Sección 6.2.

Con el fin de simular el potencial eléctrico sobre el cuero cabelludo se utilizó un modelo de cabeza realista constituido por tres capas, con superficies intermedias segmentadas en 2562 vértices cada una (5120 elementos triangulares), disponible en el programa SPM8 ${ }^{1}$ (Friston et al., 2007). Dichas capas corresponden al cerebro, cráneo y cuero cabelludo, cuyas conductividades eléctricas se supusieron $0,3 \mathrm{~S} / \mathrm{m}$, 0,02 S/m y 0,3 S/m respectivamente (Oostendorp et al., 2000; Wendel y Malmivuo, 2006; Zhang et al., 2006b). Se simularon fuentes dipolares con orientación fija y amplitud variante en el tiempo de acuerdo a la función $\nu(t)=5 \exp (-(t-$ $\left.21 / 2)^{2} / 3^{2}\right)-\exp \left(-(t-18)^{2} / 4^{2}\right)$. Se asumieron 73 electrodos dispuestos de acuerdo a una extensión del estándar 10-10 (Deuschl y Eisen, 2000). El problema directo se resolvió utilizando el BEM (Geselowitz, 1967; Sarvas, 1987), considerando el enfoque de cráneo aislado (Meijs et al., 1989). Se consideraron $N=20$ muestras temporales por realización, una relación de señal a ruido electrónico de $10 \mathrm{~dB}$ y una relación de actividad de fondo a ruido electrónico de $4 \mathrm{~dB}$.

La actividad cerebral de fondo se consideró como el potencial eléctrico generado por 500 fuentes dipolares ubicadas aleatoriamente y distribuidos uniformemente en

\footnotetext{
${ }^{1}$ Disponible en http://www.fil.ion.ucl.ac.uk/spm/
} 
el cerebro. La covarianza temporal se basó en el modelo OAM propuesto por Bijma et al. (2003) y adaptado a EEG. Los parámetros de dicho modelo se eligieron con el fin de ajustar los datos de EEG verdaderos. Debe notarse que es un modelo de ruido correlacionado temporalmente más complejo que el utilizado como esquema subyacente en el estimador de constricción. Dos conjuntos de actividad de fondo fueron creados. En el primer caso (A), se supuso estacionareidad temporal e igual potencia en cada sensor. En el segundo caso (B) no se consideró estacionareidad temporal y se contempló potencia aleatoria en cada sensor. Asimismo, se modeló una fuerte correlación entre electrodos ubicados cerca de la región occipital (en donde el ritmo Alfa es dominante) y cerca del lóbulo frontal (en donde el ritmo Delta es dominante).

En las Figuras 6.1(a) y 6.1(b) se muestran los parámetros de constricción correspondientes a la estimación de la covarianza espacial considerando los casos A y B, respectivamente. En ambas figuras, así como en todas las correspondientes a esta sub-sección, se muestra el promedio de 50 realizaciones del ruido anteriormente descripto. Se vislumbra que la diferencia entre los parámetros de constricción considerando los esquemas 5 y 6 son mayores en el caso B que en el A, como era de esperarse por la distribución desigual de la potencia de ruido en los sensores. Asimismo, se ve que el esquema 4 presenta los peores resultados, lo cual se debe a que es el único esquema con matriz de estructura subyacente de rango unitario. 


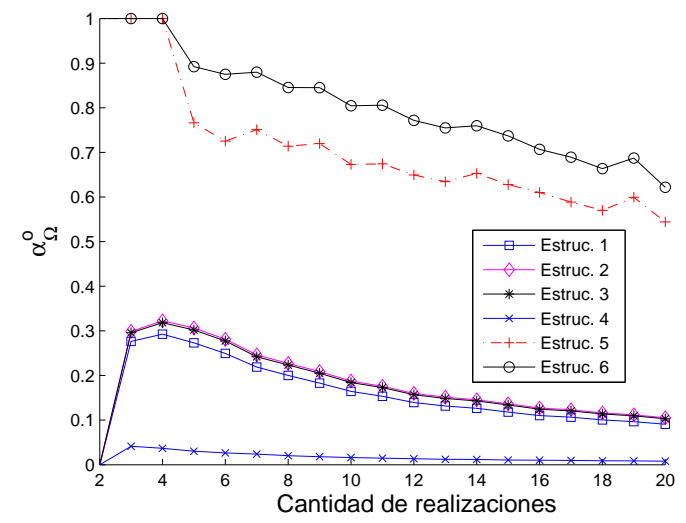

(a)

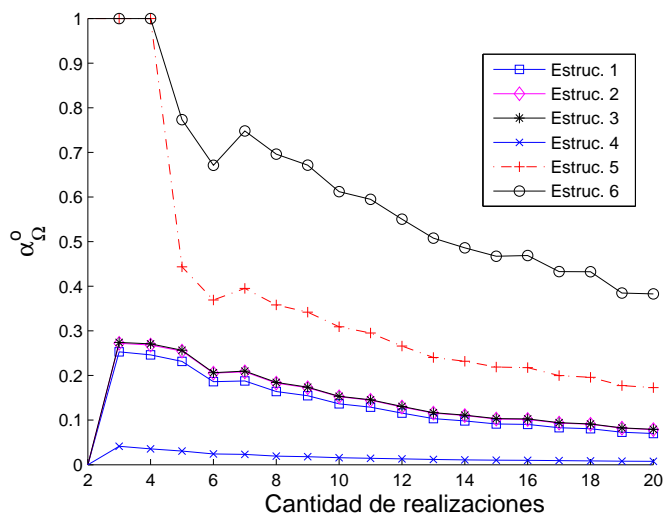

(b)

Figura 6.1: Parámetro de constricción espacial considerando los casos A (a) y B (b) (ver texto). Las diferentes curvas corresponden a las diferentes estructuras presentados en el Cuadro 6.1.

Si bien altos valores del parámetro de constricción indican una buena elección de la estructura subyacente, la relación entre este parámetro y la distancia entre las matrices de covarianza estimada y verdadera no es tan directa. Puede suceder que un estimador con estructura tal que su parámetro de constricción sea mayor a otro se encuentre más distante a la matriz de covarianza verdadera que éste. Esto puede verse en la ecuación (D.1) en el Apéndice D.1, en donde se muestra que parámetros de constricción altos pueden ser contrarrestados por pequeños valores de $\mathbb{E}\left\{\|\boldsymbol{T}-\boldsymbol{S}\|_{F}^{2}\right\}$. De todos modos, si la diferencia entre los parámetros de constricción aumenta, el factor $\mathbb{E}\left\{\|\boldsymbol{T}-\boldsymbol{S}\|_{F}^{2}\right\}$ tiende a aumentar. En las Figuras 6.2(a) y 6.2(b) se muestran las distancias normalizadas entre cada estimador y la matriz de covarianza verdadera, considerando los casos A y B, respectivamente. La distancia normalizada se calculó como $\|\boldsymbol{\Omega}-\widehat{\boldsymbol{\Omega}}\|_{F} /\|\boldsymbol{\Omega}\|_{F}$, en donde $\boldsymbol{\Omega}$ es la covarianza muestral considerando 3000 realizaciones. En la figura también se muestra la raíz cuadrada de la CRB del ECM normalizada, así como la distancia del estimador muestral (también normalizada). Puede verse que todos los estimadores propuestos superan en desempeño al estimador muestral, de acuerdo al Teorema 6.3.1. Más aún, cuando el número de realizaciones es pequeño $(\leq 6)$ y la potencia entre electrodos y muestras es constante (caso A), el estimador de constricción con la estructura 5 presenta un desempeño inclusive mejor al de la CRB, i.e. es mejor que cualquier estimador insesgado. Esto 
no sucede en el caso B, y la razón es que en este caso el estimador muestral converge más rápidamente que en el caso A, lo cual puede observarse mediante la comparación de la distancia normalizada para ambos casos. En la Figura 6.2, así como en muchas otras de la sección, se omiten las curvas correspondientes a los esquemas 1 y 3 debido a que prácticamente se superponen a la correspondiente al esquema 2.

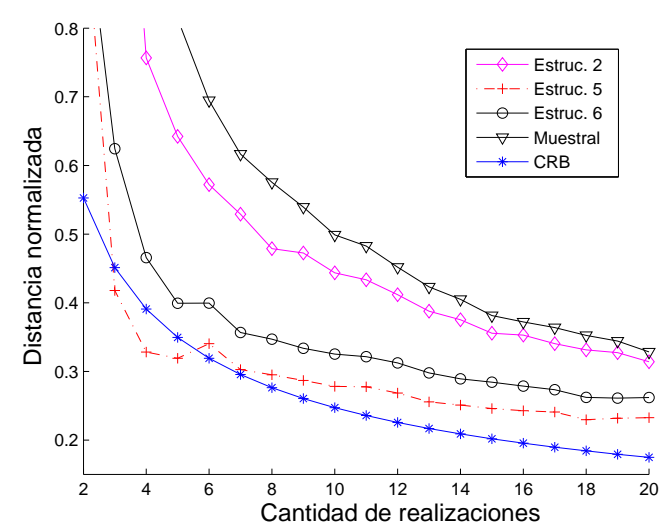

(a)

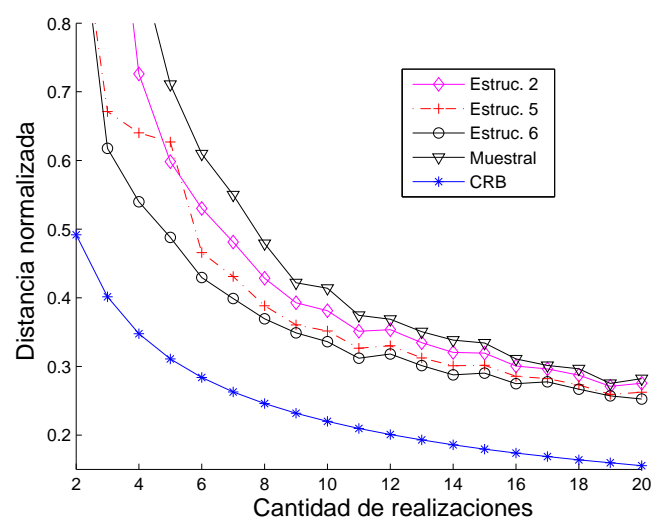

(b)

Figura 6.2: Distancia normalizada de los estimadores de constricción para la estimación de la matriz de covarianza espacial, considerando los casos A (a) y B (b) (ver texto). Las diferentes curvas corresponden a las diferentes estructuras presentados en el Cuadro 6.1. Se muestran también la raíz cuadrada normalizada de la CRB en el ECM y la distancia normalizada del estimador muestral para su comparación.

Otro parámetro de interés es el número de condición de los estimadores propuestos, y su comparación con el del estimador muestral. Como se mencionó en la Sección 6.1 la estabilidad numérica es un punto de importancia en los estimadores cuando el número de realizaciones es pequeño. En la Figura 6.3 se muestra el número de condición $c_{n}$ (en escala logarítmica) en función del número de realizaciones. El límite superior es de $10^{15}$ dado que es el límite de la precisión del programa utilizado. Los estimadores de constricción (salvo el basado en el esquema 4) presentan un mejor desempeño que el estimador muestral. Además, solo las estructuras basadas en modelos de actividad de fondo producen estimadores de la matriz de covarianza espacial no singulares en el caso de contar con 2 ó 3 realizaciones.

Se procedió de igual forma para el caso de la matriz de covarianza temporal. Dado 


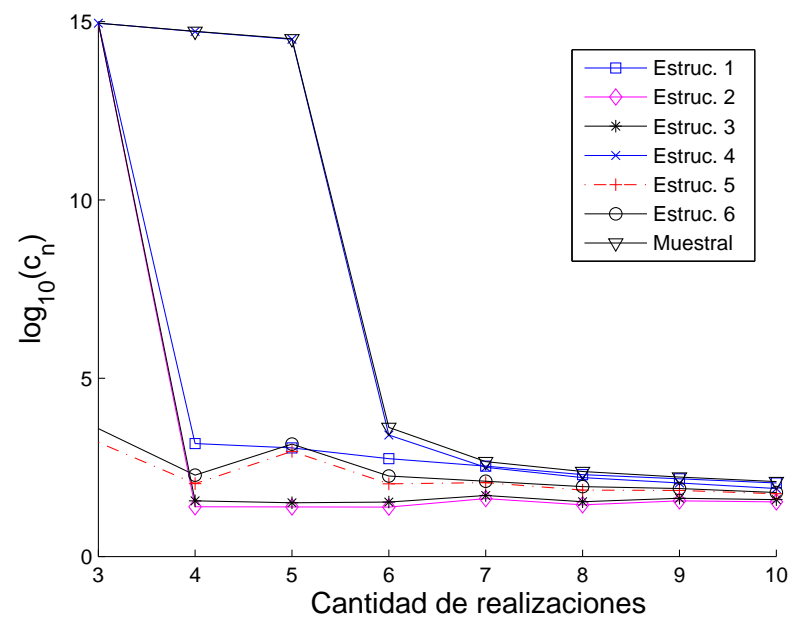

Figura 6.3: Número de condición de los estimadores de constricción en función del número de realizaciones considerado (en escala logarítmica). Las diferentes curvas corresponden a las diferentes estructuras presentados en el Cuadro 6.1.

que en este caso la estabilidad numérica del estimador muestral no es un problema (ver Sección 6.2), se espera que el estimador muestral se encuentre más cercano a la verdadera matriz de covarianza que en el caso espacial. En la Figura 6.4 se muestran las distancias normalizadas de las estructuras sesgadas utilizadas (Figura 6.4(a)) y de los estimadores de constricción (Figura 6.4(b)) a la matriz de covarianza temporal verdadera. Esta distancia se muestra como función del número de realizaciones considerado. Todas las curvas corresponden al caso de simulación B (los resultados son similares para el caso A). Asimismo, se dibujan las curvas correspondientes al estimador muestral y la CRB normalizada.

Puede verse de las figuras que, si bien los estimadores de constricción son mejores que los muestrales, dicha mejora no es de tanta importancia como en el caso de la covarianza espacial. Allí se ve que en el caso de considerar hasta 7 realizaciones de señal, los estimadores por constricción presentan un mejor desempeño que el estimador muestral, por lo que resulta recomendable. Este desempeño se presenta a partir de mayores valores del parámetro de constricción, como puede verse en la Figura 6.5, en donde se muestra el parámetro de constricción temporal en función del número de realizaciones consideradas. 


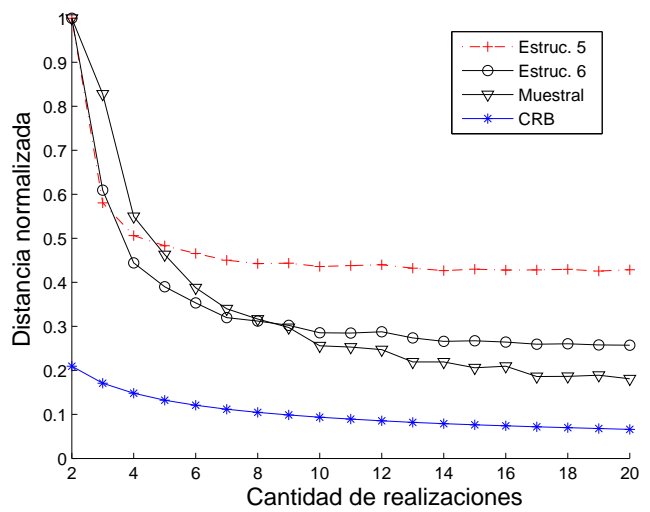

(a)

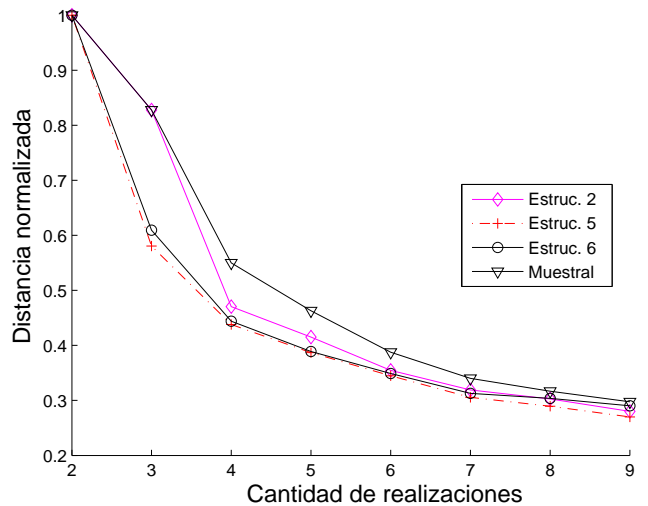

(b)

Figura 6.4: Distancia normalizada de los esquemas propuestos (a) y los estimadores de constricción (b) respecto a la verdadera matriz de covarianza, para la estimación de la matriz de covarianza temporal, considerando el caso B (ver texto). Las diferentes curvas corresponden a las diferentes estructuras presentados en el Cuadro 6.1. Se muestran también la raíz cuadrada normalizada de la CRB en el ECM y la distancia normalizada del estimador muestral para su comparación.

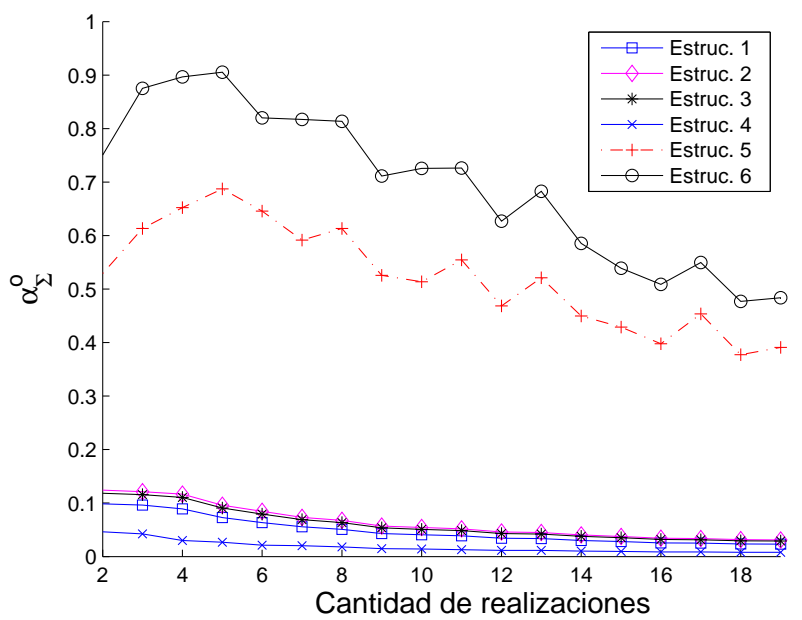

Figura 6.5: Parámetro de constricción temporal considerando datos sintéticos (caso B, ver texto). Las diferentes curvas corresponden a las diferentes estructuras presentados en el Cuadro 6.1.

\subsubsection{Resultados utilizando datos reales}

Luego, se evaluaron los estimadores de constricción de las matrices de covarianza utilizando datos reales de EEG. Como en este caso la matriz de covarianza verdadera 
es desconocida, se compararon los diferentes esquemas mediante la contrastación de los parámetros de constricción para cada uno de ellos en función del número de realizaciones consideradas. Como se mostró en la Sección 6.2, cuanto mayores sean los parámetros de constricción mejores serán los resultados (en función del ECM).

Las señales de EEG fueron adquiridas a $200 H z$ utilizando el equipo BioScience ${ }^{\circledR}$ Vector EEG64 USB, adquiriendo señales en 62 electrodos dispuestos sobre una gorra de acuerdo a una extensión del estándar 10-20 (Deuschl y Eisen, 2000), considerando la referencia promedio. El paciente resultó ser una mujer de 25 años de edad con displasia focal cortical en el lóbulo frontal. Los datos adquiridos fueron filtrados entre $0.1 \mathrm{~Hz}$ y $40 \mathrm{~Hz}$ utilizando un filtro FIR de orden 512 (Porat, 1996). Las espigas interictales fueron detectadas y extraídas de los datos filtrados y clasificadas según su forma de onda y distribución espacial. Uno de estos juegos de señales fue escogido y utilizado en este ejemplo, cuyo valor medio se muestra en la Figura 6.6. Dicho juego consistía en 14 realizaciones de 39 muestras temporales cada una.

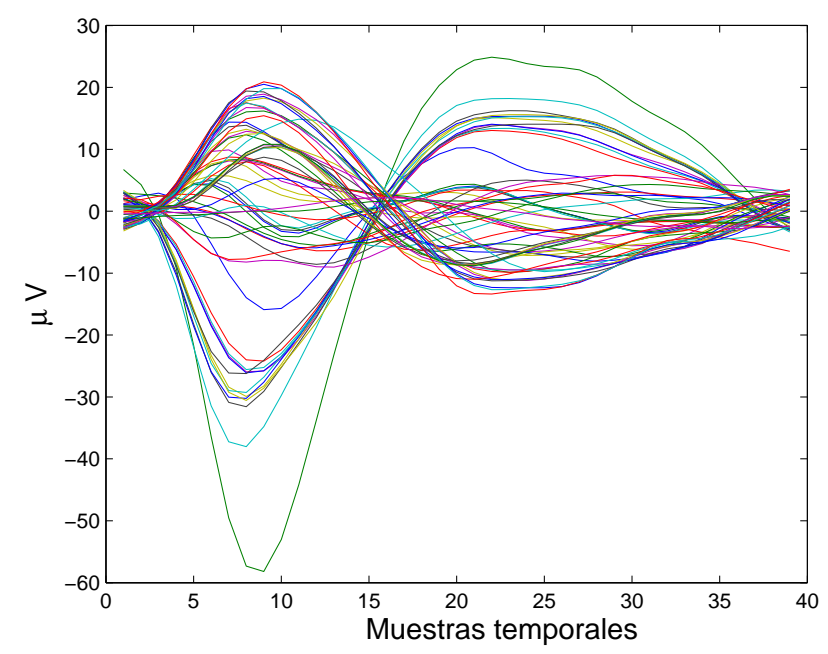

Figura 6.6: Valor medio del juego de 14 espigas consideradas para el ejemplo.

En primer lugar se evaluaron los modelos subyacentes de actividad de fondo desarrollados en la Sección 6.3.1. Para ello se calcularon los estimadores muestrales de las señales utilizando las 14 realizaciones y se las compararon con los modelos anteriores. Los resultados se muestran en las Figuras 6.7 y 6.8. Puede verse que 
los modelos desarrollados se adaptan adecuadamente a los datos, quedando además plenamente justificado el uso de los estimadores de constricción.

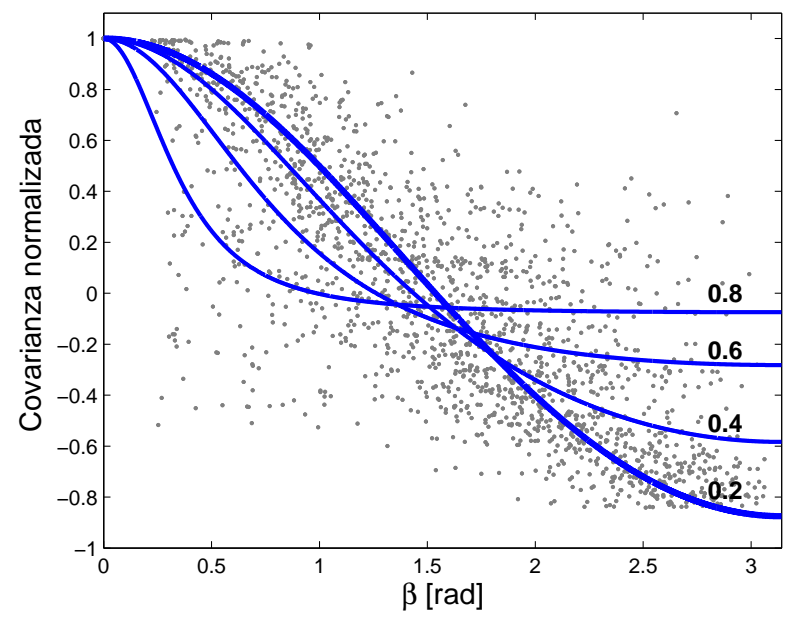

Figura 6.7: Elementos normalizados del estimador muestral de la matriz de covarianza espacial en función del ángulo entre electrodos. Los puntos grises corresponden a los datos del estimador muestral, mientras que las líneas continuas al modelo teórico representado por la ecuación (6.13) considerando $r_{0}=0.2,0.4,0.6$ y 0.8 .

Luego se calcularon los estimadores y parámetros de constricción para cada modelo presentado en el Cuadro 6.1. En las Figuras 6.9(a) y 6.9(b) se muestran los parámetros de constricción para los casos espacial y temporal, respectivamente, considerando hasta 14 realizaciones. Los resultados mostrados corresponden al valor medio de 500 diferentes estimaciones computadas mediante la consideración de distintos subconjuntos de las 14 realizaciones. La desviación estándar se omitió por claridad, pero resultó ser del orden de $2 \times 10^{-2}$ en el caso espacial y $1 \times 10^{-10}$ en el caso temporal. Puede verse en las figuras que todos los estimadores resultan ser mejores que el estimador muestral, pues los parámetros de constricción son mayores a cero. Asimismo, puede notarse que el conocimiento de la estructura subyacente permite obtener mayores parámetros de constricción. Los modelos de estructura 5 y 6 , basados en dicho conocimiento, presentan valores del parámetro de constricción mucho mayores a los obtenidos con los demás esquemas genéricos. Tal como se dijo anteriormente, una diferencia pequeña en el valor del parámetro de constricción no 


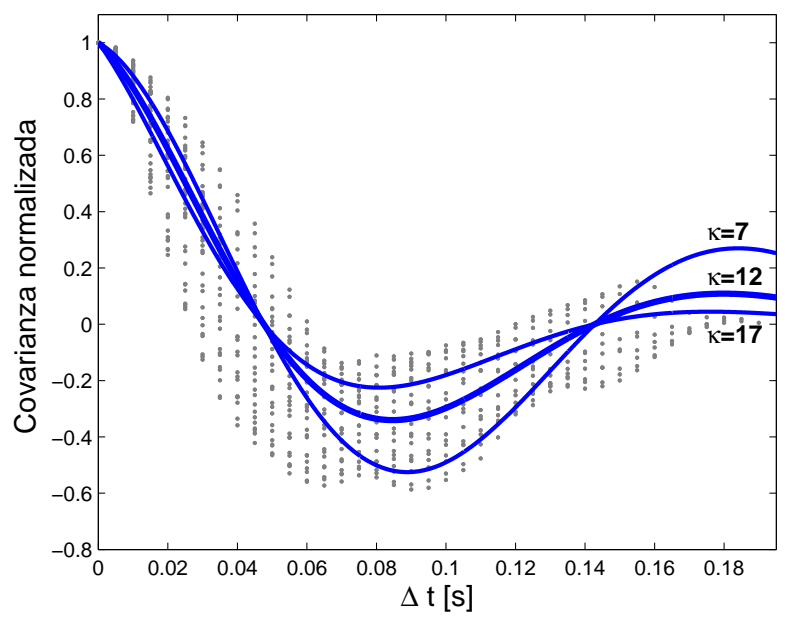

Figura 6.8: Elementos normalizados del estimador muestral de la matriz de covarianza temporal en función del tiempo entre muestras. Los puntos grises corresponden a los datos del estimador muestral, mientras que las líneas continuas al modelo teórico representado por la ecuación (6.14) considerando $\omega=33 \mathrm{rad} / \mathrm{s}$ y $\kappa=7,12$ y $17 \mathrm{~s}^{-1}$.

necesariamente indica un mejor desempeño del estimador, pero a medida que esta diferencia aumenta se vuelve un indicador de menor distancia entre las matrices de covarianza estimadas y las verdaderas.

\subsection{Discusión y conclusiones}

En este capítulo se presentó el método de constricción para la estimación de la matriz de covarianza en EEG. Estos estimadores se plantean para mitigar los problemas que presentan los estimadores comúnmente utilizados cuando el número de realizaciones es pequeño, tales como la inestabilidad numérica o el tiempo de cómputo. Se probaron algunos resultados de interés que permitieron establecer la importancia de una adecuada selección del modelo subyacente para mejorar el desempeño del método. Esto parece ser una novedad, dado que otros trabajos del área no tienen en cuenta dicho conocimiento previo. Se mostró que, en el caso de conocer información sobre la estructura de fondo de las matrices de covarianza, los estimadores propuestos presentan un mejor desempeño que los estimadores general- 


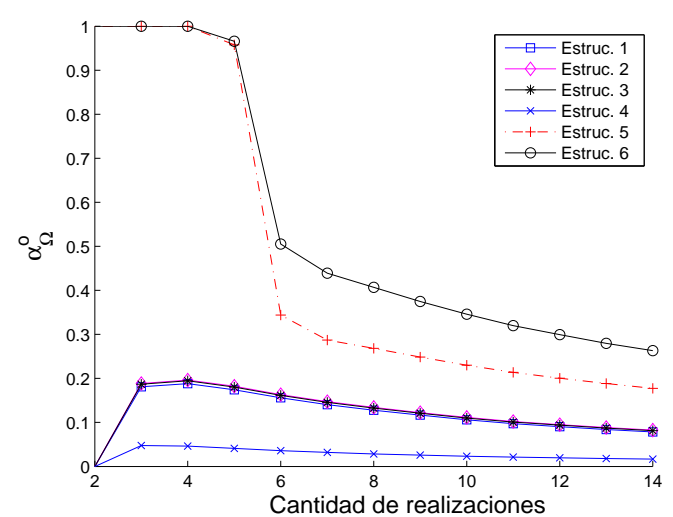

(a)

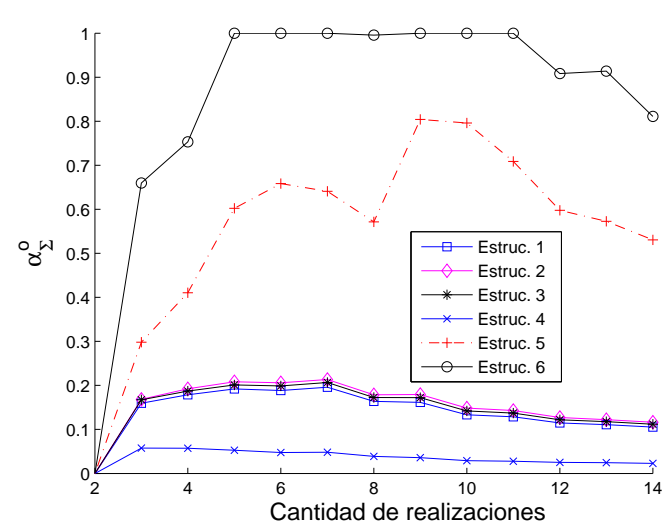

(b)

Figura 6.9: Variación del parámetro de constricción en función del número de realizaciones consideradas, para los estimadores de las matrices de covarianza espacial (a) y temporal (b). Se muestran los valores medios de 500 diferentes estimaciones seleccionando distintos subconjuntos de espigas. Las diferentes curvas corresponden a los modelos presentados en el Cuadro 6.1.

mente utilizados, especialmente si el número de realizaciones disponibles es bajo.

Se obtuvieron cotas de desempeño en la estimación de la matriz de covarianza para la métrica de ECM considerando estructura subyacente de producto de Kronecker, válido para el planteo de EEG. Ésta resulta ser una herramienta de sumo interés debido a que permite comparar el desempeño de los estimadores propuestos con el mejor estimador insesgado posible. Se aplicaron los resultados al problema de la estimación de las matrices de covarianza en EEG, concluyendo que en el supuesto de contar con potencia entre electrodos prácticamente constante y un número de realizaciones bajo (menor a 6), los estimadores de constricción aquí propuestos presentan un desempeño aún mejor al de la cota.

En el presente capítulo se presentó el método de constricción con el sentido de minimizar el ECM del estimador. Técnicamente, esto refiere a la utilización de la norma Frobenius como métrica de medida de distancia entre matrices. Como se sabe, esta métrica (también llamada plana) no es la única posible (Smith, 2005). Trabajos futuros estarán dedicados a generalizar los estimadores de constricción considerando otras métricas, tal como la natural (Smith, 2005).

Debe notarse que el presente análisis puede ser aplicado a múltiples problemas 
asociados al procesamiento estadístico de señales, pues la estimación de las matrices de covarianza de señal y ruido son partes cruciales de muchos algoritmos del área. Estos problemas pueden encontrarse relacionados a radar, beamforming, o modelado de canales para comunicaciones MIMO, en donde la estructura subyacente de producto de Kronecker se encuentra también presente. Trabajos recientes han comenzado a proponer la utilización de las técnicas aquí planteadas en dichos problemas (Stoica et al., 2008; Zhu et al., 2008; Du et al., 2009; Chen et al., 2010). Si bien estos trabajos estudian métodos eficientes de implementación de técnicas de procesamiento de señales, no toman en consideración la ventaja provista por el conocimiento de la estructura a priori.

Las estructuras aquí propuestas pueden ser utilizadas en forma adaptativa, pues los parámetros de las mismas se estiman de las realizaciones. Este hecho es de mucha importancia dado que permite lidiar con las no-estacionareidades del ruido de fondo. Esto es de sumo interés, por ejemplo, en estudios de epilepsia, pues se recomienda realizar los mismos durante el sueño (Fountain et al., 1998; Malow et al., 1999), durante el cual la actividad de fondo cambia de un modo significativo (Poepel et al., 2007). Luego, la adaptabilidad de los estimadores de constricción es de importancia en estudios de E/MEG basados en beamforming, pues en estos métodos se suele considerar mediciones de estados activo y pasivo en los que se asume que la caracterización estadística del ruido no varía en el tiempo (Sekihara y Nagarajan, 2007). Errores en la estimación de las matrices de covarianza pueden inducir un aumento de la varianza (y en algunos casos de sesgo) en la estimación de los parámetros de interés, por lo cual deben ser tenidos en cuenta.

Los estimadores de constricción también pueden ser utilizados para estudiar la dinámica entre diferentes bandas de frecuencia debido a la no-estacionareidad del ruido de fondo. Éstos pueden ser utilizados para elaborar modelos dinámicos que permitan explicar la fluctuación de potencia en el tiempo. En esta dirección se están adaptando los esquemas propuestos, como primer paso para derivar nuevos modelos con base fisiológica de la actividad de fondo. 


\section{Capítulo 7}

\section{Desempeño de los estimadores de constricción en beamforming}

\subsection{Introducción}

La resolución del PI en EEG se ve afectada por múltiples factores que limitan su desempeño, entre los que se encuentran los errores en el modelado de la cabeza (ver Capítulo 3), la incertidumbre en la posición de los electrodos (ver Capítulo 5) y las incertezas inherentes a las mediciones (Muravchik y Nehorai, 2001). Sin embargo, los efectos debidos al adecuado tratamiento de la actividad cerebral de fondo resultan ser dominantes frente a los anteriormente mencionados, por lo que el método de resolución del PI utilizado debe contemplarlo adecuadamente. Esto se logra a partir de la utilización de los momentos estadísticos que la definen, resultando ser los momentos de primer y segundo orden (i.e. la media y la covarianza) suficientes en caso de considerar una distribución Gaussiana para las señales (ver Capítulo 6, Sección 6.2.1). Estos momentos deben estimarse de las realizaciones de señal disponibles, las cuales pueden ser escasas en algunas situaciones ligadas a epilepsia, como se describe en el capítulo anterior. Para paliar estos problemas se desarrolló en el Capítulo 6 el método de estimación por constricción, el cual permite la adecuada estimación de la matriz de covarianza aún disponiendo de un escaso número de realizaciones de señal. En el presente capítulo se aplica la técnica de estimación por 
constricción en la resolución del PI en EEG, mostrando sus ventajas frente a los estimadores convencionales.

Una de las técnicas de resolución del PI en EEG que permite la consideración adecuada de la actividad de fondo es la de filtrado espacial o beamforming (van Veen et al., 1997; Robinson y Vrba, 1999; Sekihara y Nagarajan, 2007). Básicamente, el método de beamforming consiste en concentrar el arreglo de medición para el estudio de la actividad neuronal originada en una posición y en un instante determinado. En cada uno de los puntos de interés se plantea la búsqueda de la fuente que mejor representa al término de señal correspondiente a la medición, evaluando su desempeño a partir de la potencia de señal que ésta permita representar. De este modo se repite el procedimiento para todos los puntos de interés, concluyendo en un mapa de distribución de actividad cortical.

Existen dos grandes clases de beamforming disponibles en la literatura de E/MEG, los vectoriales y los escalares. Los primeros se basan en la descomposición vectorial de las componentes del momento dipolar, necesitando para ello el cálculo de tres filtros ortogonales (van Veen et al., 1997; Sekihara et al., 2001). Su principal ventaja radica en la eficiencia de cómputo para cada posición requerida, debido a que se basa en procedimientos determinísticos. En cambio, los métodos de beamforming escalar (Robinson y Vrba, 1999; Sekihara et al., 2004; Chen et al., 2006) consisten en calcular un solo filtro espacial que permite estimar la actividad en cada posición. Comparadas con el método vectorial, el método de beamforming escalar presenta mayor relación señal a ruido y mejor desempeño en la estimación de actividad neuronal (Vrba y Robinson, 2000; Sekihara et al., 2004), por lo que es el adoptado en el presente estudio.

En este capítulo se focaliza en el análisis del desempeño de una variación del método de beamforming de máximo contraste (MCB), propuesto por Chen et al. (2006). Este método, al igual que muchos de los disponibles en la literatura (Sekihara y Nagarajan, 2007), requiere de dos clases de mediciones a realizarse. Una de ellas denominada de control, en la que se miden realizaciones del ruido de fondo solamente. La otra, denominada de actividad, consiste en la medición de realizaciones de la señal de interés, como ser espigas interictales en el caso de epilepsia. Luego, 
suponiendo que la actividad de fondo no cambia en el tiempo y es la misma en ambas modalidades de medición, se calculan los filtros escalares que permiten determinar la fuente de actividad cerebral. Como se dijo en el capítulo anterior, esto presenta serias limitaciones. En principio, se necesita de mediciones de control que permitan la adecuada representación del ruido, las cuales no siempre se encuentran disponibles. Más aún, existe evidencia sobre la variabilidad de la actividad de fondo en el tiempo (Poepel et al., 2007), especialmente sobre algunas bandas de frecuencia en momentos de actividad patológica (Telenczuk et al., 2011), variando ésta de acuerdo a la medicación suministrada al paciente (Tan, 1989). Luego, resulta necesario basar el análisis en los datos disponibles, a partir de los cuales se debe caracterizar adecuadamente el ruido de fondo.

Por esta razón se propone el uso del método de constricción desarrollado en el capítulo anterior. Tal como se mostró anteriormente, este método permite la caracterización del ruido de fondo de forma adecuada aún en circunstancias en las que se poseen pocas realizaciones de señal. Se muestran las ventajas del método en la formulación teórica del problema, reduciendo a sólo uno el número de restricciones impuesto originalmente.

El presente capítulo se organiza del siguiente modo: en la Sección 7.2 presenta el método de MCB y sus limitaciones, tras lo cual se propone el uso de los estimadores por constricción de la matriz de covarianza, resultando en el método de beamforming por constricción. Luego, en la Sección 7.3 se presentan resultados utilizando datos sintéticos generados a partir de un modelo realista, los cuales permiten vislumbrar las ventajas del método propuesto. Finalmente, en la Sección 6.4 se presentan conclusiones del capítulo y líneas de trabajo futuro.

\subsection{Métodos}

En la siguiente sección se presenta el MCB adaptado al conocimiento de señales de actividad solamente. Luego, se plantea el uso de los estimadores de constricción y sus ventajas en relación al número de realizaciones disponible. 


\subsubsection{Beamforming de máximo contraste}

En el presente análisis se considera el modelo de señal presentado en la Sección 6.2.1 del capítulo anterior. Es decir, se considera que el fenómeno bajo estudio es $\boldsymbol{Y} \sim \mathcal{N}_{P \times N}(\boldsymbol{M}, \boldsymbol{\Sigma}, \boldsymbol{\Omega})$, en donde $\boldsymbol{Y}$ es la matriz aleatoria de $P \times N, \boldsymbol{M}$ es la media (asociada al término de señal), y $\boldsymbol{\Sigma}$ y $\boldsymbol{\Omega}$ son las matrices de covarianza temporal y espacial, respectivamente. Esto resulta equivalente a asumir un modelo de la forma $\boldsymbol{Y}=\boldsymbol{M}+\boldsymbol{R}$, en donde $\boldsymbol{M}$ es el término de señal, determinístico, y $\boldsymbol{R} \sim \mathcal{N}_{P \times N}(\mathbf{0}, \boldsymbol{\Sigma}, \boldsymbol{\Omega})$ es el término de ruido de fondo. Asimismo, se considera que se miden $K$ realizaciones de $\boldsymbol{Y}$, siendo $\boldsymbol{Y}^{k}$ la $k$-ésima. Dado que se supone que la señal es la misma en cada iteración, $\boldsymbol{M}$ será independiente de $k$, conllevando a $\boldsymbol{Y}^{k}=\boldsymbol{M}+\boldsymbol{R}^{k}$, en donde $\boldsymbol{R}^{k}$ es la $k$-ésima realización del ruido. Si se supone que la actividad cerebral de interés se debe a una fuente dipolar, el término de señal puede representarse mediante $\boldsymbol{M}=\boldsymbol{l} \boldsymbol{s}^{T}$, en donde $\boldsymbol{l}=\boldsymbol{L}_{q} \boldsymbol{q}$ es el vector de señal en los $P$ sensores considerando una fuente de intensidad unitaria, $\boldsymbol{L}_{q}$ es la matriz de lead-field correspondiente a dicha fuente, de momento $\boldsymbol{q}$, y $\boldsymbol{s}$ es el vector de $N \times 1$ con elementos que representan la amplitud del momento dipolar en los $N$ instantes de tiempo considerados en cada realización.

El método de MCB consiste básicamente en buscar un vector de pesos $\boldsymbol{w}$ de dimensión $P \times 1$ de modo que la salida del filtro, $\boldsymbol{z}^{T}=\boldsymbol{w}^{T} \boldsymbol{Y}$, represente del mejor modo a la amplitud de la fuente en los $N$ instantes de tiempo. Utilizando el modelo de señal se tiene que

$$
\boldsymbol{z}^{T}=\boldsymbol{w}^{T} \boldsymbol{Y}=\boldsymbol{w}^{T} \boldsymbol{M}+\boldsymbol{w}^{T} \boldsymbol{R}=\boldsymbol{w}^{T} \boldsymbol{l} \boldsymbol{s}^{T}+\boldsymbol{w}^{T} \boldsymbol{R},
$$

por lo que el vector de pesos buscados resulta de minimizar la varianza del ruido de la salida del filtro imponiendo $\boldsymbol{w}^{T} \boldsymbol{l}=1$. Matemáticamente el problema queda expresado por

$$
\widehat{\boldsymbol{w}}=\arg \min _{\boldsymbol{w}}\left[\mathbb{E}\left\{\left(\boldsymbol{w}^{T} \boldsymbol{R}\right)\left(\boldsymbol{w}^{T} \boldsymbol{R}\right)^{T}\right\}\right] \text { sujeto a } \boldsymbol{w}^{T} \boldsymbol{l}=1,
$$

cuya solución resulta ser (Chen et al., 2006)

$$
\widehat{\boldsymbol{w}}=\rho \boldsymbol{\Omega}^{-1} \boldsymbol{l},
$$


en donde $\rho$ es un factor de proporcionalidad constante. Debe notarse que el vector de pesos buscado depende del momento dipolar de la fuente, por lo que se lo debe estimar previo a la determinación del vector de pesos. Para ello se propone evaluar un funcional en cada punto de interés que permita determinar el aporte de una fuente dipolar en dicha ubicación a la señal resultante, desconsiderando adecuadamente el ruido de fondo. En forma similar (pero no igual) a Chen et al. (2006) se plantea minimizar la relación entre la potencia de señal adquirida respecto de la de ruido solamente, i.e.

$$
F=\frac{\mathbb{E}\left\{\left\|\boldsymbol{w}^{T} \boldsymbol{Y}\right\|^{2}\right\}}{\mathbb{E}\left\{\left\|\boldsymbol{w}^{T} \boldsymbol{Q}\right\|^{2}\right\}}=\frac{\boldsymbol{w}^{T} \boldsymbol{C}_{Y} \boldsymbol{w}}{\boldsymbol{w}^{T} \boldsymbol{\Omega} \boldsymbol{w}},
$$

en donde $\boldsymbol{C}_{Y}=\mathbb{E}\left\{\boldsymbol{Y} \boldsymbol{Y}^{T}\right\}$. Luego, se busca el momento dipolar $\boldsymbol{q}$ que maximice $F$. Reemplazando (7.3) en (7.4) se tiene

$$
F=\frac{\boldsymbol{l}^{T} \boldsymbol{\Omega}^{-1} \boldsymbol{C}_{Y} \boldsymbol{\Omega}^{-1} \boldsymbol{l}}{\boldsymbol{l}^{T} \boldsymbol{\Omega}^{-1} \boldsymbol{l}}=\frac{\boldsymbol{q}^{T} \boldsymbol{L}_{q}^{T} \boldsymbol{\Omega}^{-1} \boldsymbol{C}_{Y} \boldsymbol{\Omega}^{-1} \boldsymbol{L}_{q} \boldsymbol{q}}{\boldsymbol{q}^{T} \boldsymbol{L}_{q}^{T} \boldsymbol{\Omega}^{-1} \boldsymbol{L}_{q} \boldsymbol{q}}=\frac{\boldsymbol{q}^{T} \boldsymbol{P} \boldsymbol{q}}{\boldsymbol{q}^{T} \boldsymbol{Q} \boldsymbol{q}},
$$

en donde $\boldsymbol{P}=\boldsymbol{L}_{q}^{T} \boldsymbol{\Omega}^{-1} \boldsymbol{C}_{Y} \boldsymbol{\Omega}^{-1} \boldsymbol{L}_{q}$ y $\boldsymbol{Q}=\boldsymbol{L}_{q}^{T} \boldsymbol{\Omega}^{-1} \boldsymbol{L}_{q}$. Finalmente, el estimador del momento dipolar vendrá dado por (Chen et al., 2006)

$$
\widehat{\boldsymbol{q}}=\arg \operatorname{máx}_{\boldsymbol{q}} \frac{\boldsymbol{q}^{T} \boldsymbol{P} \boldsymbol{q}}{\boldsymbol{q}^{T} \boldsymbol{Q q}}
$$

cuya solución resulta ser el autovector correspondiente al máximo autovalor de la matriz $\boldsymbol{Q}^{-1} \boldsymbol{P}$ (Horn y Johnson, 1985).

Ciertas diferencias deben destacarse entre el planteo realizado en Chen et al. (2006) y el presente. En principio, y tal como se detallara en la Sección 7.1, el planteo original del MCB presupone la medición de señales de modo activo y control, las cuales no se suponen en el presente. Por otro lado, la estimación de $\boldsymbol{w}$ aquí planteada no necesitó de la imposición de restricciones sobre su norma, como ocurre en Chen et al. (2006). Esta restricción conlleva a la variabilidad de resultados de acuerdo al valor impuesto sobre la misma, sin encontrar argumentos sólidos en la determinación de un valor fijo (Chen et al., 2006). 


\subsubsection{Beamforming por constricción}

Una vez planteado el método resulta necesario estimar las matrices de covarianza para resolver el problema planteado. Para ello se propone el uso de los estimadores por constricción de las matrices de covarianza, los cuales mostraron ser estimadores acertados con mejor desempeño que los utilizados comúnmente en condiciones de disponibilidad de un escaso número de realizaciones, sin la necesidad de realizar mediciones de control ni suposición alguna.

En el presente análisis se consideraron dos entornos de estimación posibles. En el primero, denominado de acá en mas Caso 1, se consideró la estimación por constricción de $\Omega$ tal cual fue planteada en el capítulo anterior. En el segundo, denotado Caso 2, se consideró la utilización del estimador de la matriz de covarianza temporal, no tenida en cuenta en el primer caso. Más explícitamente, se planteó realizar un pre-blanqueado temporal de los datos para luego obtener el estimador de la covarianza espacial. Esto se realiza con el fin de evaluar la forma en que la reducción de la presencia de información sistemática no relevante impacta en la estimación de $F$ y $\boldsymbol{q}$. Este pre-blanqueado consiste en la multiplicación de $\boldsymbol{Y}$ a derecha por $\boldsymbol{\Sigma}^{-1 / 2}$, en donde $\boldsymbol{\Sigma}^{-1}=\boldsymbol{\Sigma}^{-1 / 2} \boldsymbol{\Sigma}^{-T / 2}$ es la descomposición de Cholesky. Luego, $\boldsymbol{Y}_{n}=\boldsymbol{l} \boldsymbol{s}_{n}^{T}+\boldsymbol{Q}_{n}$, en donde $\boldsymbol{Y}_{n}=\boldsymbol{Y} \boldsymbol{\Sigma}^{-1 / 2}, \boldsymbol{Q}_{n}=\boldsymbol{Q} \boldsymbol{\Sigma}^{-1 / 2}$ y $\boldsymbol{s}_{n}=\boldsymbol{\Sigma}^{-T / 2} \boldsymbol{s}$. Dado que $\mathbb{E}\left\{\boldsymbol{Q} \boldsymbol{\Sigma}^{-1} \boldsymbol{Q}^{T}\right\}=N \boldsymbol{\Omega}$ (ver Kollo y von Rosen (2005), Teorema 2.2.9, p.207), el problema es similar al anterior, en donde se considera otra amplitud de señal, pero la ubicación de la fuente y el momento dipolar no cambian. Sin embargo, pueden esperarse cambios en la estimación muestral del parámetro de constricción espacial, debido a que la estimación de $\boldsymbol{\Sigma}$ se obtiene con menor error numérico que $\boldsymbol{\Omega}$, pudiendo afectar en situaciones de bajo número de realizaciones.

\subsection{Resultados}

En esta sección se presentan resultados correspondientes a la resolución del PI en EEG mediante el método de beamforming por constricción. Para ello se utilizó el modelo de cabeza realista utilizado en el capítulo anterior (Sección 6.4.2). Para la 
resolución del PI se consideró una fuente dipolar fija en una región del cerebro con $90 \%$ de probabilidad de corresponder a una zona de materia gris, de acuerdo al atlas ICBM-152. Para contar con mayor resolución en la localización de la fuente se consideró una región de interés de $15 \mathrm{~mm}$ alrededor de la fuente, en donde se calculó el lead-field en puntos equidistantes separados en $1 \mathrm{~mm}$.

La señal simulada presentó variación temporal dada por $\nu(t)=5 \exp (-(t-$ $\left.21 / 2)^{2} / 3^{2}\right)-\exp \left(-(t-18)^{2} / 4^{2}\right)$, contaminada con ruido correlacionado espaciotemporalmente. La covarianza espacial se obtuvo de la actividad generada por 400 fuentes dipolares dispuestas en forma aleatoria, mientras que la covarianza temporal se consideró igual a la correspondiente a las señales reales, mostrada en la Figura 6.14 del Capítulo 6, limitado a 20 muestras por realización. En cada uno de las figuras mostradas en la presente sección se muestra el valor medio y la desviación estándar (cuando corresponda) considerando 100 realizaciones diferentes del ruido.

En el presente análisis se consideró la comparación de dos de los esquemas propuestos en el Capítulo 6, los modelos 2 (aquí llamado LW) y 6 (aquí llamado MF). Asimismo, se consideró adecuado, también con fines de comparación, explicitar resultados correspondientes a la utilización del estimador muestral y la matriz identidad. Este último es propuesto por Chen et al. (2006) en situaciones en las que la estimación del ruido se vuelve dificultosa. En la Figura 7.1 se muestra el error en la localización y en la estimación del momento dipolar en función del número de realizaciones, considerando los esquemas y casos de simulación mencionados.

Puede verse la notable ventaja de los estimadores de constricción frente a los generalmente utilizados o propuestos, resultando aún mejor cuando el caso de simulación 2 se consideró, i.e. cuando se realiza un pre-blanqueado temporal. Estos resultados se mantienen en la estimación de la localización y del momento dipolar, logrando resultados por demás aceptables. Debe notarse que el máximo error cometido corresponde a $15 \mathrm{~mm}$ debido a la precisión espacial considerada. Resulta de interés comparar las ventajas de la consideración del pre-blanqueado mediante una comparación directa entre los casos de simulación 1 y 2 y los esquemas LW y MF. En la Figura 7.2 se muestra esta comparación, marcando la media y la desviación estándar en función del número de realizaciones. 

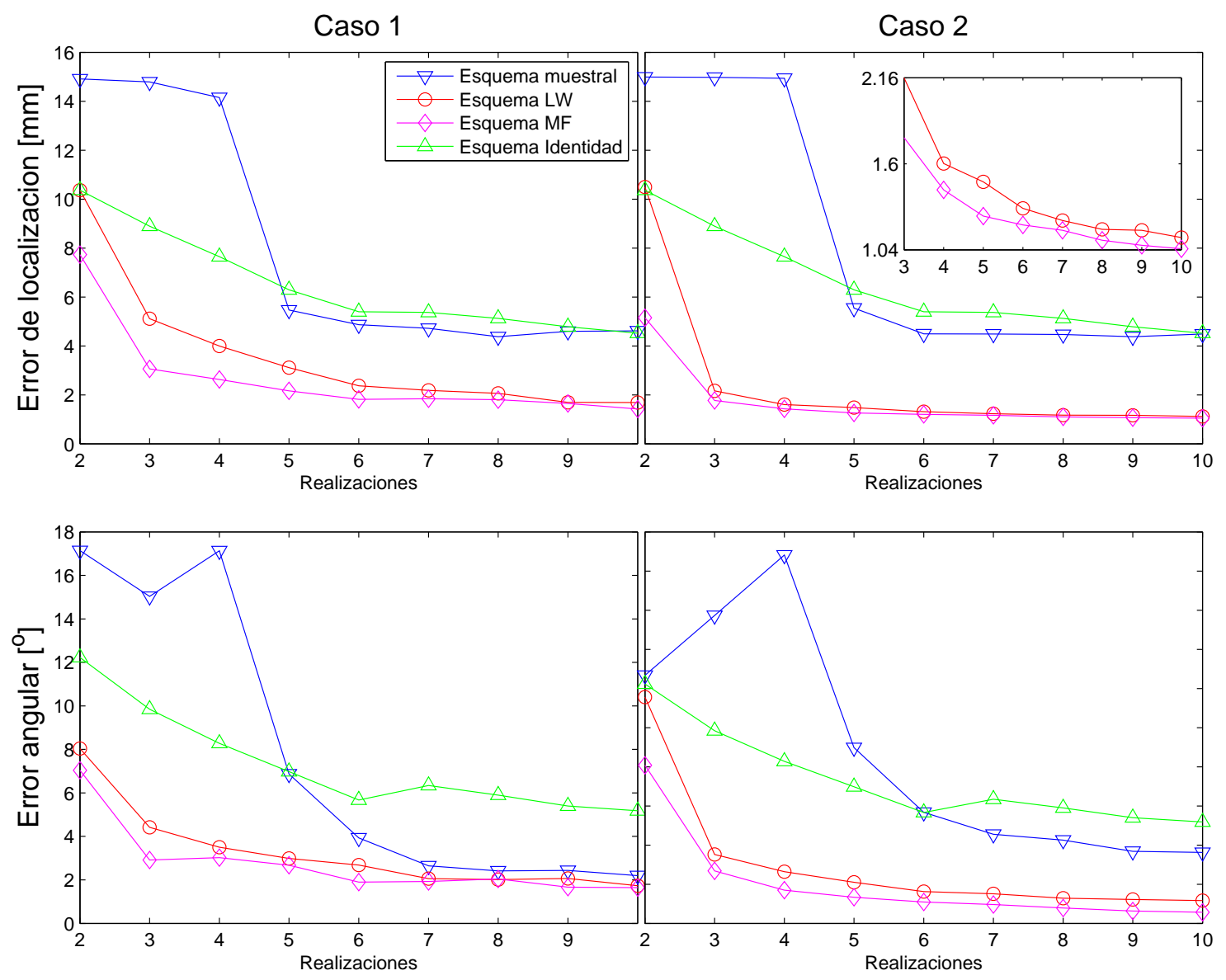

Figura 7.1: Error en la localización $[\mathrm{mm}]$ y angular $\left[^{\circ}\right]$ en función del número de realizaciones considerado. Se muestran resultados para los casos de simulación 1 (izquierda) y 2 (derecha), utilizando los esquemas propuestos (ver texto).

Se ve claramente que el caso de simulación 2 no solo permite obtener mejores estimadores de la posición y momento de la fuente, sino que permite obtener menor dispersión de los valores estimados. Para comprender las razones de ello se calculó la distancia normalizada entre las matrices de covarianza espacial estimadas y la matriz verdadera, calculada esta última como el estimador muestral considerando 3000 realizaciones. Esta distancia normalizada se calculó como $d_{i}=\left\|\boldsymbol{C}_{i} /\right\| \boldsymbol{C}_{i}\left\|_{F}-\boldsymbol{C} /\right\| \boldsymbol{C}\left\|_{F}\right\|_{F}$, en donde $\boldsymbol{C}_{i}$ es el estimador de la matriz $\boldsymbol{C}$. Esta medida de error, similar al NRDM, resulta de interés en el presente problema, pues los resultados son independientes del factor de magnitud $\rho$. 

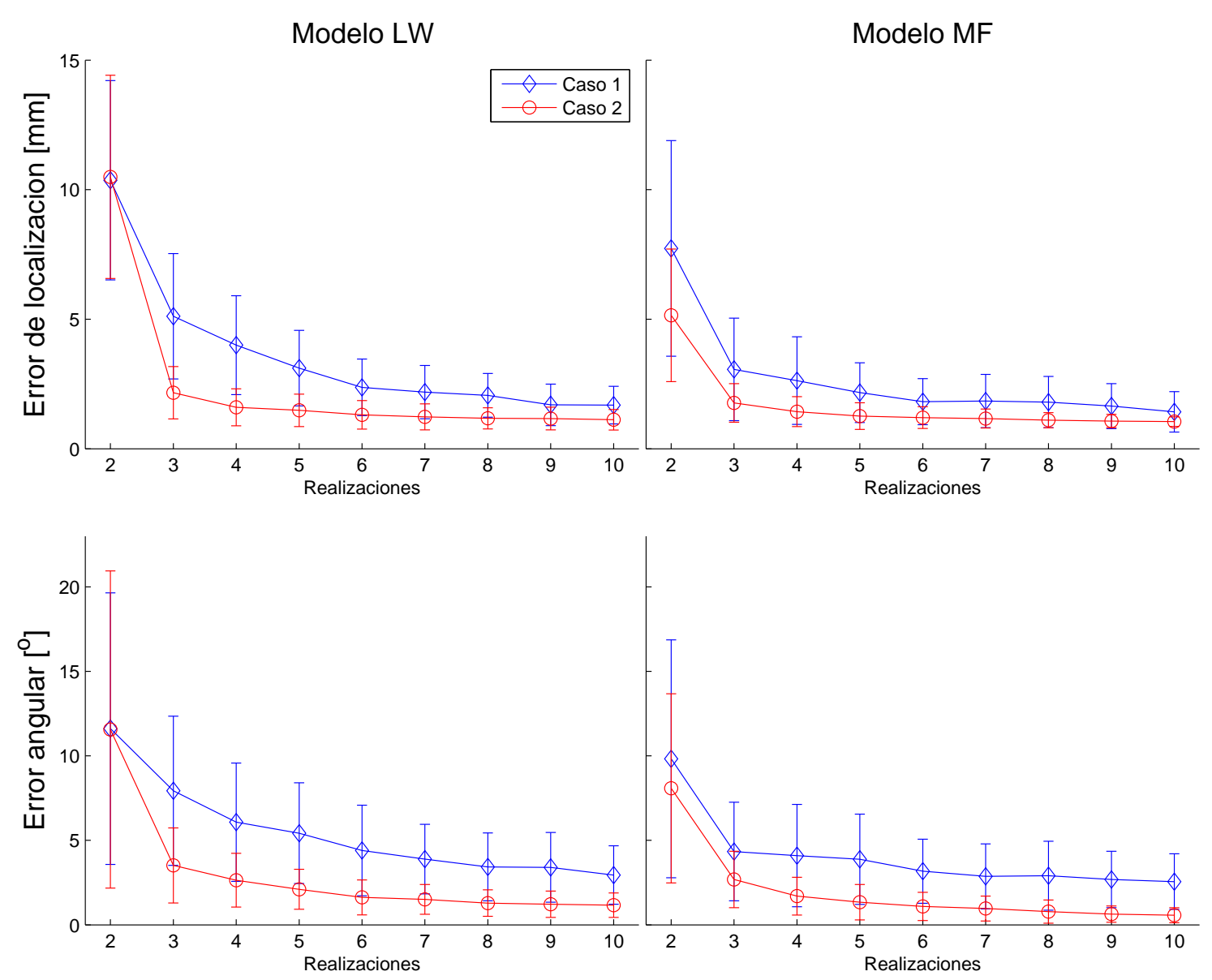

Figura 7.2: Error en la localización $[\mathrm{mm}]$ y angular $\left[^{\circ}\right]$ en función del número de realizaciones considerado. Se muestran resultados para los casos de simulación 1 y 2 (con distintos marcadores), utilizando los esquemas LW (izquierda) y MF (derecha). Asimismo, se presenta la desviación estándar en las realizaciones de simulación (100).

Se ve claramente que la distancia entre los estimadores y la matriz de covarianza verdadera ratifican los resultados anteriores, resultando de mejores estimadores en el caso de simulación 2 (al menos para su aplicación en beamforming).

\subsection{Discusión y conclusiones}

En el presente capítulo se evaluaron las técnicas de estimación de matrices de covarianza por constricción en la resolución del PI de EEG mediante beamforming 


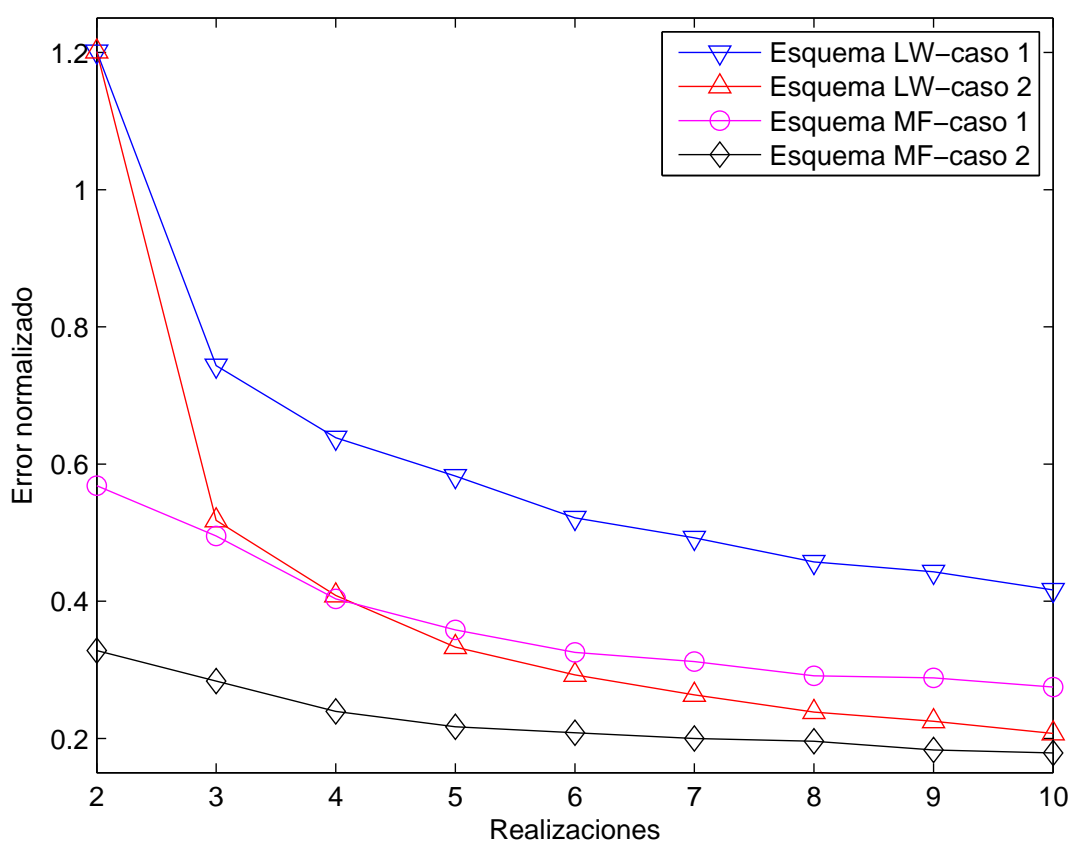

Figura 7.3: Distancia normalizada entre los esquemas LW y MF respecto de la matriz de covarianza verdadera, considerando los casos de simulación 1 y 2.

escalar. El uso de estos estimadores permitió reformular el método de MCB con la finalidad de resolver el PI sin la necesidad de mediciones en estado de control. Esto resulta un hecho de notoria importancia en los métodos de filtrado espacial aplicados en E/MEG, pues reduce las limitaciones presentadas por dichos algoritmos. Asimismo, el uso de estimadores de constricción permitió la utilización de este método de resolución del PI aún contando con un número acotado de realizaciones, siendo habitual en estudios relacionados a epilepsia.

Se plantearon dos situaciones para la estimación de la matriz de covarianza del ruido. En la primera se propuso la estimación de la matriz de covarianza espacial de acuerdo a lo explicado en el capítulo anterior. En la segunda se propuso preblanquear temporalmente la señal de entrada utilizando los estimadores de constricción de la matriz de covarianza temporal. Si bien se probó que los resultados no varían en condiciones de infinita cantidad de realizaciones, su consideración permitió obtener mejores estimadores muestrales de los parámetros de constricción, resultando en mejores estimadores y, por ende, resultados más exactos. Esto se debe 
a que su estimación resulta numéricamente estable, permitiendo descartar factores variables que no aportan a la estimación de la covarianza espacial. Asimismo, y tal como se recalcó en el capítulo precedente, el costo computacional propio de los estimadores propuestos es sumamente bajo (fracciones de segundo), por lo que su uso resulta altamente recomendable.

La aplicación del método de constricción en beamforming está siendo actualmente objeto de múltiples estudios relacionados al procesamiento de señales por arreglos de sensores (Stoica et al., 2008; Yang et al., 2009; Chen et al., 2010; Gu y Leshem, 2011; Huang y Lee, 2012) en algunas aplicaciones directas (Zhang et al., 2009). Sin embargo, como se aclaró en el capítulo anterior, el estudio de la influencia de la adecuada selección de la matriz de sesgo en la estimación de la matriz de covarianza y, por ende, en sus aplicaciones, no ha sido abordado en detalle. En este capítulo se mostraron las virtudes de ello en un problema de aplicación directa en neurociencias, destacando la importancia de su estudio.

El planteo de resolución aquí propuesto presenta las ventajas esperables en la resolución del PI mediante beamforming. Sin embargo, resultan no del todo explícitas las razones que ligan al pre-blanqueo temporal con un mejor estimador de constricción. Si bien las razones parecen ser las explicadas anteriormente, estudios futuros estarán destinados a la cuantificación explícita de las mismas. Asimismo, trabajos posteriores estarán destinados a la comparación respecto de distintos métodos de resolución del PI, así como su aplicación utilizando datos reales. 


\section{Capítulo 8}

\section{Influencia del ruido en imágenes de tensor de difusión}

\subsection{Introducción}

La resonancia magnética por tensor de difusión (RM-TD) es una técnica que permite caracterizar a los tejidos biológicos in vivo y de forma no invasiva (Basser et al., 1994; Pierpaoli et al., 1996). Básicamente consiste en la medición de la difusividad de las moléculas de agua a lo largo de múltiples direcciones con la finalidad de estudiar la movilidad de los protones de hidrógeno en función de la orientación. Dado que la difusión en los tejidos no es libre, el agua se difundirá más rápidamente en la dirección alineada con la estructura interna y más lentamente en la dirección perpendicular a membrana que lo contiene, permitiendo la obtención de información acerca de su estructura anatómica. Entre muchas otras aplicaciones, RM-TD permite caracterizar la estructura fibrilar de la materia blanca y proveer de mapas de conectividad cerebral que ayudan en el entendimiento de su funcionamiento (Hagmann et al., 2003).

Si se supone una densidad de probabilidad Gaussiana para el fenómeno de difusión, esta movilidad estará matemáticamente dada por el tensor de difusión (TD) (Moseley et al., 1990). Más explícitamente, el TD es una herramienta que permite caracterizar a la movilidad del agua en cada punto del volumen bajo análisis a partir 
de un elipsoide que represente del mejor modo la difusividad del agua en función de la dirección. Dicho elipsoide permite caracterizar el nivel de difusión en cada una de las direcciones de los semiejes a partir de su longitud. Luego, un elipsoide esférico indica igual difusión en todas las direcciones, uno oblato (i.e. tipo disco) indicará difusión prioritaria en sólo dos de ellas y uno prolato (i.e. tipo fibra) en sólo una. Matemáticamente dicho elipsoide quedará determinado por el TD, el cual no es más que una matriz de $3 \times 3$ cuyos autovectores y autovalores definen la dirección y longitud de sus semiejes. A partir de un número de mediciones se busca estimar al tensor de difusión en los voxels del volumen considerando algún criterio (Basser et al., 1994; Niethammer et al., 2006; Landman et al., 2007).

Una vez estimados los TD del volumen de interés surgen múltiples aplicaciones. Una de ellas es la cuantificación de la anisotropía de la materia blanca mediante el cálculo de factores escalares muy utilizados en la práctica clínica, tales como la anisotropía fraccional (FA) o la anisotropía relativa (RA). Su cálculo permite, por ejemplo, realizar diferentes diagnósticos médicos, como ocurre con varios desórdenes del sistema nervioso central (Sorensen et al., 1999; Bammer et al., 2000; Filippi et al., 2001). Asimismo, la estimación del TD es utilizada en la reconstrucción fibrilar del cerebro in vivo (Mori et al., 1999; Basser et al., 2000; Lazar et al., 2003), la cual sirve para probar el grado de invasividad de los neoplasmas en tractos funcionales (Mori et al., 1999) y dilucidar la conectividad funcional y anatómica (Koch et al., 2002), entre tantos otros usos. Existen también otras aplicaciones del estimador del TD, tal como el cálculo del mapa de tensores de conductividad eléctrica (Tuch et al., 2001) que permite la consideración de modelos de cabeza realistas para la resolución adecuada de los problemas directo e inverso en E/MEG (Wolters et al., 2006; Güllmar et al., 2010).

Puesto que las mediciones de RM-TD se encuentran inevitablemente contaminadas con ruido, toda función del tensor de difusión tendrá una varianza asociada. Si bien ésta puede ser reducida mediante la manipulación del sistema adquisidor, existen límites de desempeño teóricos a partir de los cuales no podrá mejorarse la estimación propuesta. Luego, la caracterización de dichos límites resulta de sumo interés en la comunidad científica, pues permiten determinar los alcances del estu- 
dio y establecer la configuración del sistema a fin de lograrlos. Con tal finalidad en algunos trabajos (Alexander, 1997; Caan et al., 2010) se ha propuesto la utilización de la cota de Cramér-Rao (CRB) con el objeto de establecer la mínima varianza conseguible con un estimador insesgado del tensor de difusión y funciones de éste. Sin embargo, los planteos existentes no toman en consideración a los esquemas de adquisición de múltiples bobinas, sólo estudiados en el marco de RM-TD en contadas publicaciones (Gilbert et al., 2007; Trisán-Vega et al., 2009; Aja-Fernández et al., 2010a; Giannelli et al., 2011). En este capítulo se plantea un modelo de adquisición de señales de RM-TD considerando múltiples bobinas receptoras. Para ello se adapta el modelo de RM paralela (RMp) ampliamente utilizado en la práctica de RM (Roemer et al., 1990; Larkman y Nunes, 2007), tras lo cual se generaliza el cálculo de la CRB considerando dicha técnica de adquisición. Esto permite estudiar los límites teóricos en la estimación tanto del tensor de difusión como de funciones de éste, como ser parámetros escalares que denoten el grado de anisotropía del mismo y el autovector principal, con consecuencias inmediatas en la reconstrucción fibrilar del cerebro. Asimismo, posibilita evaluar la necesidad de la consideración de múltiples bobinas adquisidoras en RM-TD y la variación de los límites teóricos en función del número de receptores de señal.

El presente capítulo se organiza del siguiente modo: en la Sección 8.2 se presenta el modelo de señal adoptado en RM-TD bajo la consideración de múltiples bobinas adquisidoras. Luego se obtienen expresiones del límite en el desempeño en la estimación del TD y funciones de éste. En la Sección 8.3 se muestran resultados correspondientes a la CRB considerando datos sintéticos y reales. Los primeros permiten analizar la influencia de ciertos parámetros en la estimación del TD, como su forma, el número y dirección de los gradientes de campo aplicados y el número de bobinas adquisidoras. En cambio, la consideración de datos reales permite establecer el grado de influencia certero de algunos de los parámetros bajo estudio y su distribución espacial. Finalmente, en la Sección 8.4 se presentan los alcances e implicancias de los resultados, conclusiones del capítulo y líneas de trabajo futuro. 


\subsection{Métodos}

En esta sección se presentan los fundamentos del estudio. En principio se formula el modelo de adquisición de señales de RM-TD en paralelo, basado en el modelo de RMp. Luego se presenta la CRB como metodología para cuantificar la influencia del ruido en dichas mediciones, para finalmente considerar la calidad de la estimación de algunos parámetros de interés dependientes del TD frente al ruido.

\subsubsection{Modelo de señal}

Algunas de las mayores limitaciones en aplicaciones de RM-TD recaen en la limitada resolución espacial y relación señal a ruido (SNR) conseguibles con un sistema de adquisición constituido por una única bobina receptora. Por esta razón sistemas con múltiples bobinas planteados originalmente en RM (Roemer et al., 1990) fueron adaptados a RM-TD con el fin de obtener mejoras en la resolución espacial e incrementar la SNR (Bammer et al., 2002; Jaermann et al., 2004, 2008).

\section{Modelo de señal de RM paralela}

Las señales de RM son generalmente modeladas como un proceso complejo Gaussiano, en donde la parte real e imaginaria de la señal original se encuentran contaminadas con ruido Gaussiano no correlacionado de media nula e igual varianza $\sigma^{2}$. En muchos trabajos (e.g. Constantinides et al. (1997)) se estudian las características de dicho ruido considerando sistemas de una sola bobina, concluyendo que es razonable considerar que la señal recibida es una variable aleatoria con distribución Rice en caso de comprender señal y ruido, y Rayleigh en caso de comprender solo ruido. Estos modelos son importantes en múltiples aplicaciones, como en la estimación de la SNR o la relación contraste a ruido (CNR), entre otras (Gudbjartsson y Patz, 1995; Constantinides et al., 1997; Landman et al., 2009).

Sin embargo, los sistemas de adquisición de una única bobina cuentan con algunas limitaciones, como ser la velocidad en la adquisición de datos y la SNR conseguible. Para paliar estos problemas se propusieron sistemas de adquisición de múltiples 
bobinas (Roemer et al., 1990; Larkman y Nunes, 2007), tras lo cual surgieron diferentes métodos de fusión de la información medida por cada una de ellas. Si se busca mejorar la SNR y el montaje de bobinas es completamente conocido, el filtro espacialmente adaptado es la opción recomendada (Dietrich et al., 2008). Por otro lado, si el montaje ya se encuentra caracterizado y la intención es aumentar la velocidad de adquisición algunos métodos sugeridos son el algoritmo generalizado auto-calibrado de adquisición paralela (GRAPPA) (Griswold et al., 2002) en el dominio de la frecuencia, y el método de sensibilidad codificada (SENSE) (Pruessmann et al., 1999) en el dominio de la imagen.

No obstante, a pesar de que estos métodos son los recomendables, no es común en la práctica médica el conocimiento de la caracterización completa del sistema de adquisición utilizado, así como tampoco es común el acceso a la configuración del resonador. Luego, en estos casos el método de suma-de-cuadrados (SoS) es el recomendado (Roemer et al., 1990; Larsson et al., 2003; Larkman y Nunes, 2007), y el considerado en el presente capítulo.

Sea $S_{l}$ la señal compleja medida por la l-ésima bobina, y $A_{l}$ la señal compleja ideal sin ruido ni atenuación en la misma bobina. Luego, si no se asume acoplamiento alguno entre bobinas adquisidoras, el modelo

$$
S_{l}=\rho_{l}(\boldsymbol{p}) A_{l}+\xi
$$

resulta válido, en donde $\rho_{l}(\boldsymbol{p})$ es la sensibilidad de la l-ésima bobina en el punto $\boldsymbol{p}$ del volumen bajo estudio, y $\xi$ es una variable aleatoria compleja Gaussiana de media nula y varianza $\sigma^{2}$. Luego, la magnitud de salida del SoS de un sistema compuesto por $L$ bobinas será

$$
S_{T}=\sqrt{\sum_{l=1}^{L}\left|S_{l}\right|^{2}}
$$

Se muestra fácilmente que, en presencia de señal, $S_{T}$ es una variable aleatoria con distribución chi no-central (Aja-Fernández et al., 2010b), con función densidad de probabilidad

$$
f\left(S_{T} ; A_{T}, \sigma, L\right)=\frac{A_{T}^{1-L}}{\sigma^{2}} S_{T}^{L} \exp \left(-\frac{S_{T}^{2}+A_{T}^{2}}{2 \sigma^{2}}\right) I_{L-1}\left(\frac{A_{T} S_{T}}{\sigma^{2}}\right),
$$


en donde $A_{T}=\left(\sum_{l=1}^{L}\left|\rho_{l}(\boldsymbol{p}) A_{l}\right|^{2}\right)^{1 / 2}$ e $I_{\alpha}(\cdot)$ es la función de Bessel modificada de primera especie y orden $\alpha$. Es preciso notar que (8.2) se reduce a una distribución Rice en el caso de que un sistema de una sola bobina sea utilizado.

Debe aclararse que un planteo general de SoS debería contemplar diferencias entre las varianzas de cada bobina, como se marca en Aja-Fernández et al. (2010b), conllevando a una distribución chi no-central generalizada (Mallik, 2003). Sin embargo, en situaciones prácticas la varianza del ruido es lo suficientemente homogénea respecto a los píxeles y las bobinas, por lo que la consideración de la distribución chi no-central es adecuada cuando la señal se reconstruye con SoS y GRAPPA (Dietrich et al., 2008), avalando la selección del modelo propuesto.

\section{Modelo de señal de RM-TD paralelo}

Tal como se mencionó anteriormente, RM-TD es una técnica que permite medir la anisotropía del medio y caracterizar la estructura interna del cerebro in vivo. Esto se realiza mediante la aplicación de un gradiente de campo magnético en un número de direcciones, tras lo cual la atenuación de señal en cada voxel es medida. Luego, las mediciones dependerán de la secuencia de gradientes aplicada, su dirección e intensidad. Muchos de estos parámetros se encuentran comprendidos en el factor $b$, el cual determina la escala de las mediciones y depende de la intensidad y dirección del gradiente de campo (LeBihan, 1991). Si se utiliza la secuencia convencional PGSE (Stejskal y Tanner, 1965) el tiempo de difusión vendrá dado por $\Delta-\delta / 3$, en donde $\Delta$ es el tiempo entre gradientes y $\delta$ es la duración de cada uno de ellos. Luego, el factor $b$ viene dado por (Skare, 2002)

$$
b=\gamma^{2} \delta^{2}\left(\Delta-\frac{\delta}{3}\right) g_{a}^{2}
$$

en donde $\gamma$ es la constante giromagnética y $g_{a}$ la amplitud del gradiente de campo aplicado. Valores usuales de $b$ oscilan entre $1000 \mathrm{~s} / \mathrm{mm}^{2}$ y $3000 \mathrm{~s} / \mathrm{mm}^{2}$.

En el caso de considerar que el fenómeno de difusión se encuentra distribuido de forma Gaussiana, la intensidad de la señal medida por la l-ésima bobina cuando se 
aplica el n-ésimo gradiente de campo se encuentra dada por (Larsson et al., 2003)

$$
S_{l n}=\rho_{l}(\boldsymbol{p}) A_{n}+\xi
$$

en donde $A_{n}=S_{0} \exp \left(-\boldsymbol{g}_{n}^{T} \boldsymbol{\theta}\right), S_{0}$ es la señal medida sin gradiente de campo aplicado, $\boldsymbol{g}_{n}$ es un vector de 6 elementos dependiente de la dirección del gradiente aplicado y del factor $b$, y $\boldsymbol{\theta}=\left[\theta_{1}, \cdots, \theta_{6}\right]^{T}$ es el vector de las 6 componentes que definen al tensor de difusión (véase el Apéndice E.1 por las definiciones de $\boldsymbol{g}_{n}$ y $\boldsymbol{\theta}$ y Skare (2002) por la fundamentación del modelo). Debe notarse que en el presente caso sólo es de interés la intensidad de la señal y no su fase, por lo que la intensidad de la señal sin ruido variará entre bobinas dependiendo sólo de su patrón de sensibilidad.

Si se considera independencia entre bobinas (i.e. bobinas totalmente desacopladas) y la misma desviación estándar para cada medición, el método de SoS puede ser utilizado, tras lo cual las mediciones se encontrarán distribuidas de acuerdo a (8.2). Debe notarse que, por depender la señal sin ruido medida por cada bobina del perfil de sensibilidad del sistema de adquisición, ésta dependerá también del esquema de gradiente aplicado y del tensor de difusión medido en cada voxel. Utilizando definiciones previas se muestra fácilmente que la salida de señal del SoS cuando el $n$-ésimo gradiente de campo es aplicado tiene una función distribución de probabilidad $f\left(S_{T n} ; A_{T n}, \sigma, L\right)$, con $S_{T n}=\left(\sum_{l=1}^{L}\left|S_{l n}\right|^{2}\right)^{1 / 2}, A_{T n}=C_{L}(\boldsymbol{p}) S_{0} \exp \left(-\boldsymbol{g}_{n}^{T} \boldsymbol{\theta}\right) \mathrm{y}$ $C_{L}(\boldsymbol{p})=\left(\sum_{l=1}^{L} \rho_{l}^{2}(\boldsymbol{p})\right)^{1 / 2}$

\subsubsection{Cota de Cramér-Rao}

El principal objetivo del presente capítulo es caracterizar la influencia del error de las mediciones en la estimación del tensor de difusión y funciones de éste. Dado que se busca un análisis independiente del algoritmo de estimación utilizado se propone el uso de la CRB. Sea $g(\boldsymbol{\theta})$ una función del parámetro $\boldsymbol{\theta}$ a estimar. Luego, la CRB establece que (Kay, 1993)

$$
\mathbb{E}\left\{(g(\boldsymbol{\theta})-\widehat{g}(\boldsymbol{\theta}))(g(\boldsymbol{\theta})-\widehat{g}(\boldsymbol{\theta}))^{T}\right\} \geq \mathbf{C R B}_{g}=\frac{\partial g(\boldsymbol{\theta})^{T}}{\partial \boldsymbol{\theta}} \boldsymbol{J}^{-1} \frac{\partial g(\boldsymbol{\theta})}{\partial \boldsymbol{\theta}}
$$

en donde $\mathbf{C R B}_{g}$ es la matriz que representa a la cota, $\boldsymbol{J}$ es la matriz de información de Fisher y la desigualdad implica que la diferencia entre ambas matrices es positiva 
semi-definida. Si se considera un sistema de adquisición compuesto por $L$ bobinas receptoras, en el Apéndice E.1 se muestra que

$$
\boldsymbol{J}=C_{L}^{2}(\boldsymbol{p}) \boldsymbol{G}_{\eta} \Upsilon \boldsymbol{G}_{\eta}^{T}
$$

en donde $\boldsymbol{G}_{\eta}$ es una matriz dependiente del esquema de gradientes de campo, del factor $b$ y de la SNR, y $\Upsilon$ es una matriz diagonal dependiente en el factor de sensibilidad $C_{L}(\boldsymbol{p})$ y de la SNR. En función de estos resultados es fácil ver como $\boldsymbol{J}$ se reduce al caso Rice (Alexander, 1997) cuando se utiliza un sistema con una única bobina adquisidora.

Una vez calculada $\boldsymbol{J}$, su uso para el cálculo de $\mathbf{C R B}_{g}$ es directo. Cambiando la función $g(\boldsymbol{\theta})$ pueden evaluarse distintas cotas en el desempeño teórico de funciones del tensor de difusión, tales como la FA, RA o bien sus autovalores y autovectores. A continuación se presentan algunos de ellos.

\section{Mínimo error cuadrático medio}

Sea $\boldsymbol{D}$ el tensor de difusión bajo análisis. Dado que $\boldsymbol{D}$ es una matriz de $3 \times 3$, se vuelve necesaria la definición de una métrica que permita cuantificar la distancia entre cualquier estimador $\widehat{\boldsymbol{D}}$ del tensor de difusión y su verdadero valor. Luego, el error cuadrático medio (ECM) bajo la norma Frobenius parece ser una elección adecuada (Smith, 2005). Este error se define como

$$
\operatorname{ECM}(\widehat{\boldsymbol{D}})=\mathbb{E}\left\{\|\widehat{\boldsymbol{D}}-\boldsymbol{D}\|_{F}^{2}\right\}
$$

tras lo cual es fácilmente visible que esta métrica tiende a cero a medida que la distancia entre los tensores es menor.

Con el objeto de comparar el mínimo ECM conseguible por cualquier estimador debe notarse que

$$
\begin{aligned}
\operatorname{ECM}(\widehat{\boldsymbol{D}}) & =\mathbb{E}\left\{\|\widehat{\boldsymbol{D}}-\boldsymbol{D}\|_{F}^{2}\right\}=\sum_{i, j=1}^{3} \mathbb{E}\left\{\left(D_{i j}-\widehat{D_{i j}}\right)^{2}\right\}=\sum_{i, j=1}^{3} \operatorname{Var}\left\{\widehat{D_{i j}}\right\} \\
& \geq \sum_{i, j=1}^{3} C R B\left(D_{i j}\right)=\sum_{k=1}^{6} \xi_{k} C R B_{k k}=\operatorname{EC} M_{\text {mín }}(\widehat{\boldsymbol{D}}),
\end{aligned}
$$


en donde $E C M_{\text {mín }}(\widehat{\boldsymbol{D}})$ es el mínimo ECM que puede lograrse con un estimador insesgado de $\boldsymbol{D}$, y $\xi_{k}$ es un vector que vale 1 si $k=1,2,3$ y 2 si $k=4,5,6$. Luego, a partir de (8.5) es posible caracterizar el mínimo error en la estimación del tensor de difusión y su variación con los parámetros del sistema.

\section{Mínima desviación estándar en la estimación de parámetros escalares}

Tal como se mencionó anteriormente, la caracterización de los tensores de difusión mediante factores escalares resulta de gran interés. Estos factores pueden ser los comúnmente utilizados RA y FA, ampliamente adoptados por la comunidad científica, u otros factores alternativos, como puede ser la relación de área elipsoidal (EAR) (Xu et al., 2009), el cual viene ganando adeptos (Afzali y Soltanian-Zadeh, 2010; Kang et al., 2010; Hayes et al., 2011). Éstos se definen por (Xu et al., 2009)

$$
\begin{aligned}
& R A=\sqrt{\frac{\left(\lambda_{1}-\lambda_{2}\right)^{2}+\left(\lambda_{1}-\lambda_{3}\right)^{2}+\left(\lambda_{2}-\lambda_{3}\right)^{2}}{\left(\lambda_{1}+\lambda_{2}+\lambda_{3}\right)^{2}}}, \\
& F A=\sqrt{\frac{\left(\lambda_{1}-\lambda_{2}\right)^{2}+\left(\lambda_{1}-\lambda_{3}\right)^{2}+\left(\lambda_{2}-\lambda_{3}\right)^{2}}{2\left(\lambda_{1}^{2}+\lambda_{2}^{2}+\lambda_{3}^{2}\right)}}, \\
& E A R=1-\sqrt[p]{\frac{\lambda_{1}^{p} \lambda_{2}^{p}+\lambda_{1}^{p} \lambda_{3}^{p}+\lambda_{2}^{p} \lambda_{3}^{p}}{3 \lambda_{M}^{2 p}}},
\end{aligned}
$$

en donde $\lambda_{1} \geq \lambda_{2} \geq \lambda_{3}$ son los autovalores de $\boldsymbol{D}$ ordenados en forma decreciente, y $p=1,6075$.

Con el objeto de comparar el error en la estimación de éstos para su posterior recomendación de uso se plantea calcular la CRB, que permitirá evaluar la menor desviación estándar en la estimación de cada uno de ellos. Para calcularla, de acuerdo a (8.3), es necesario evaluar las derivadas de dichas funciones con respecto a $\boldsymbol{\theta}$, para lo que se necesitan calcular las derivadas de los autovalores en función de los elementos del tensor. Esto se muestra en el Apéndice E.2.

\section{Mínimo error en la estimación del autovector principal}

Tal como se mencionó en la Sección 8.1 la caracterización del autovector asociado al mayor autovalor del tensor de difusión, aquí denominado autovector principal, re- 
sulta de sumo interés en métodos de tractografía basados en dicho autovector (Mori et al., 1999; Basser et al., 2000), muy utilizadas en clínica neurológica por su disponibilidad en numerosas aplicaciones informáticas. El interés del siguiente análisis recae en la caracterización del ángulo de error cometido en la determinación del autovector principal y en la influencia de la cantidad de gradientes de campo y de bobinas adquisidoras en dicho ángulo.

Sea $\boldsymbol{q}$ el autovector principal del tensor de difusión $\boldsymbol{D}$. En Hasan et al. (2001) se muestra que éste viene dado por $\boldsymbol{q}=\boldsymbol{a} /\|\boldsymbol{a}\|$, en donde

$$
\boldsymbol{a}=\left[\left(\theta_{4} \theta_{6}-B \theta_{5}\right)\left(\theta_{5} \theta_{6}-C \theta_{4}\right),\left(\theta_{5} \theta_{6}-C \theta_{4}\right)\left(\theta_{5} \theta_{4}-A \theta_{6}\right),\left(\theta_{4} \theta_{6}-B \theta_{5}\right)\left(\theta_{5} \theta_{4}-A \theta_{6}\right)\right]^{T},
$$

$A=\theta_{1}-\lambda_{1}, B=\theta_{2}-\lambda_{1}$ y $C=\theta_{3}-\lambda_{1}$. A partir de esta expresión resulta factible el cálculo de la $\mathrm{CRB}$ en la estimación del autovector principal, $\mathbf{C R B}_{\mathbf{q}}$, mediante la consideración de $g(\boldsymbol{\theta})=\boldsymbol{q}$ en la ecuación (8.3).

Sea $\widehat{\boldsymbol{q}}$ un estimador insesgado de $\boldsymbol{q}$. Es posible mostrar que la matriz de covarianza de dicho estimador será (Koay et al., 2008; Jeong y Anderson, 2008)

$$
\Sigma_{\mathbf{q}}=\omega_{1} \boldsymbol{c}_{1} \boldsymbol{c}_{1}^{T}+\omega_{2} \boldsymbol{c}_{2} \boldsymbol{c}_{2}^{T}+0 \boldsymbol{q} \boldsymbol{q}^{T}
$$

en donde $\boldsymbol{c}_{1}$ y $\boldsymbol{c}_{2}$ son los autovectores de $\boldsymbol{\Sigma}_{\mathbf{q}}$ correspondientes a los autovalores $\omega_{1}$ y $\omega_{2}$, respectivamente. La expresión anterior implica que $\boldsymbol{\Sigma}_{\mathbf{q}}$ es de rango 2 por no presentar variación en la dirección del autovector mismo, por lo que presentará variaciones solo en el plano normal a éste.

En el caso de suponer $\widehat{\boldsymbol{q}} \sim \mathcal{N}\left(\boldsymbol{q}, \boldsymbol{\Sigma}_{\mathbf{q}}\right)$, es posible determinar el elipsoide que contiene al estimador con una cierta probabilidad $P$. Se sabe que la probabilidad de que el estimador de $\boldsymbol{q}$ se encuentre encerrado en el elipsoide $\boldsymbol{x}^{T} \boldsymbol{\Sigma}_{\mathbf{q}}{ }^{-1} \boldsymbol{x}=c^{2}$ es (van Trees, 2001)

$$
P=1-\frac{K}{\Gamma\left(\frac{K}{2}+1\right) 2^{K / 2}} \int_{c}^{\infty} x^{K} \exp \left(-x^{2} / 2\right) d x,
$$

en donde $K$ es la dimensión del elipsoide (3 en el presente caso) y $\Gamma(\cdot)$ es la función Gamma. Asimismo, se sabe que dicho elipsoide de concentración encierra (o a lo sumo es igual) al elipsoide $\boldsymbol{x}^{T} \mathbf{C R B}_{\mathbf{q}}{ }^{-1} \boldsymbol{x}=c^{2}$, constituyendo el mismo un límite teórico en la estimación, sin importar el algoritmo utilizado (van Trees, 2001). Este elipsoide, definido por la matriz $c^{2} \mathbf{C R B}_{\mathbf{q}}$, tendrá semiejes en las direcciones de sus 
autovectores, cada uno de longitud igual a la raíz cuadrada de sus autovalores. Luego, dado que el autovalor de $\mathbf{C R B}_{\mathbf{q}}$ correspondiente a la dirección $\boldsymbol{q}$ es cero, el lugar geométrico que denota el límite teórico de concentración con probabilidad $P$ será la elipse de semiejes $c \sqrt{\omega_{1}^{C R}}$ y $c \sqrt{\omega_{2}^{C R}}$, en donde $\omega_{1}^{C R}$ y $\omega_{2}^{C R}$ son los autovalores no nulos de $\mathbf{C R B}_{\mathbf{q}}$. Este lugar geométrico suele figurarse como un cono de variación del autovector principal (Koay et al., 2008), como se observa en la Figura 8.1. En el presente análisis, dicho cono representará la mínima región en donde encontrar al estimador del autovector principal con probabilidad $P$.

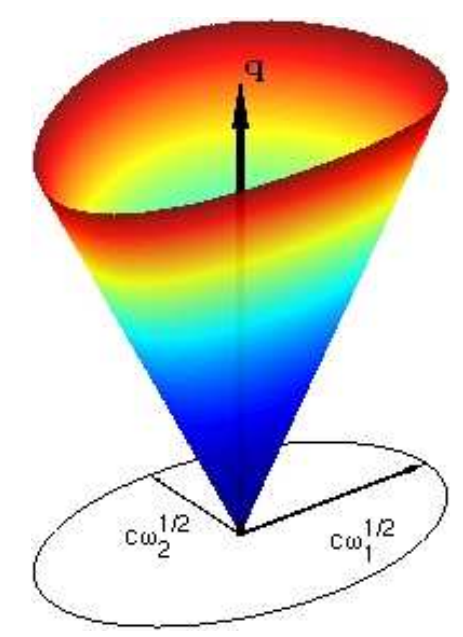

Figura 8.1: Esquema representativo del cono de concentración de $95 \%$. Se detalla tanto el cono y el autovector correspondiente como así también la elipse de error sobre el plano tangente a dicho vector (ver texto).

Dado que es de interés el cálculo del mínimo ángulo de concentración de probabilidad $P$, es fácil calcularlo mediante

$$
\alpha_{P}=\operatorname{atan}\left(c \operatorname{máx}_{j} \sqrt{\omega_{j}^{C R}}\right) .
$$

Luego, si se considera $P=0,95, \alpha_{95}$ representará al (mínimo) ángulo de concentración de $95 \%$ (de probabilidad). 


\subsection{Resultados}

En esta sección se hace uso de la formulación anteriormente desarrollada con el fin de evaluar la influencia del ruido de medición en la estimación del tensor de difusión y funciones de éste. En principio se muestran resultados utilizando datos sintéticos con el fin de evaluar la influencia del ruido en la estimación y su dependencia con el esquema de gradientes utilizado, el número de bobinas adquisidoras, la forma y orientación del tensor, la relación señal a ruido y el factor $b$. Luego, se muestra esta influencia sobre imágenes de RM-TD reales y determinan condiciones de medición necesarias para una adecuada estimación. Salvo excepciones explícitas en el texto, se consideraron los valores típicos $b=1200 \mathrm{~s} / \mathrm{mm}^{2}$ y $S N R=30$. Asimismo, la sensibilidad de cada bobina adquisidora se consideró homogénea en el volumen de interés (Dietrich et al., 2008).

En la presente sección se tomaron los coeficientes de variación porcentuales como medidas de error, e.g. si $A$ es una magnitud dependiente del tensor y $\sigma_{A}^{m i n}$ es la mínima desviación estándar en la estimación de $A$ (dada por la CRB), el coeficiente de variación porcentual vendrá dado por

$$
e_{A}=\frac{\sigma_{A}^{\min }}{A} \times 100
$$

Debe aclararse que se definió $e_{E C M}=E C M_{\text {min }}^{1 / 2}(\widehat{\boldsymbol{D}}) /\|\boldsymbol{D}\|_{F} \times 100$ solo por comodidad (y abuso) en la notación.

\subsubsection{Resultados utilizando datos sintéticos}

Con la finalidad de determinar el desempeño en la estimación del TD y funciones de éste se procedió a considerar datos sintéticos que permitiesen la variación controlada de su forma y orientación. Estas variaciones permiten estudiar la influencia de la naturaleza del tensor en su posterior estimación. Para ello se simularon TD con diferentes formas y aproximadamente 1000 orientaciones uniformemente distribuidas sobre un hemisferio de una esfera. 


\section{Comparación de esquemas de gradiente de campo}

En principio se resolvió utilizar el ECM con el fin de evaluar diferentes esquemas de gradiente de campo. Existen numerosos planteos en la bibliografía, de los cuales algunos son óptimos en algún sentido. En Skare et al. (2000) se propone un esquema cuya finalidad es la minimización del número de condición de la matriz de transformación que relaciona al tensor de difusión con los coeficientes de difusión aparente; en Poonawalla y Zhou (2004) se plantea el uso de un esquema que contempla igual ángulo sólido entre direcciones; en Jones et al. (1999) se aplica la teoría de fuerzas de repulsión electrostática para calcular configuraciones de gradientes óptimas; y en Koay et al. (2011) se propone la utilización de gradientes uniformemente distribuidos sobre la superficie de una esfera unitaria.

En la Figura 8.2 se presenta un diagrama de cajas (boxplot) que permite efectuar la comparación del $e_{E C M}$ considerando distintos números de gradientes y esquemas propuestos. En dicha figura se muestran los resultados correspondientes a los esquemas propuestos en 1: Poonawalla y Zhou (2004), 2: Skare et al. (2000), 3: Jones et al. (1999) y 4: Koay et al. (2011), considerando $N=6$ en a., $N=10$ en b., $N=30$ en c. y $N=40$ en d. Se consideraron tensores del tipo fibra $\left(\lambda_{1}=1 \times 10^{-3} \mathrm{~mm}^{2} / \mathrm{s}\right.$ y $\left.\lambda_{2}=\lambda_{3}=0,1 \times 10^{-3} \mathrm{~mm}^{2} / \mathrm{s}\right)$ y una sola bobina adquisidora.

De la comparación entre los esquemas seleccionados se ve claramente que el 3 y 4 ofrecen un mejor desempeño teórico en la reconstrucción del TD considerando la métrica del ECM, obteniendo tanto menor valor de mediana (línea roja dentro de las cajas) como mayor uniformidad espacial (extremos por fuera de las cajas). Esto es deseable pues a priori puede no suponerse conocida la distribución de direcciones de los TD, logrando de esta manera uniformidad en el error de estimación sin importar la orientación del mismo.

Del igual modo resulta factible la comparación del mínimo ángulo de concentración de $95 \%$ utilizando cada esquema. Éste permite evaluar a cada disposición de direcciones de gradiente de campo en la estimación del autovector principal, por lo que resulta de sumo interés en estudios de RM-TD cuya finalidad última sea estimar la estructura fibrilar del cerebro. Los resultados se muestran en la Figura 8.3, 


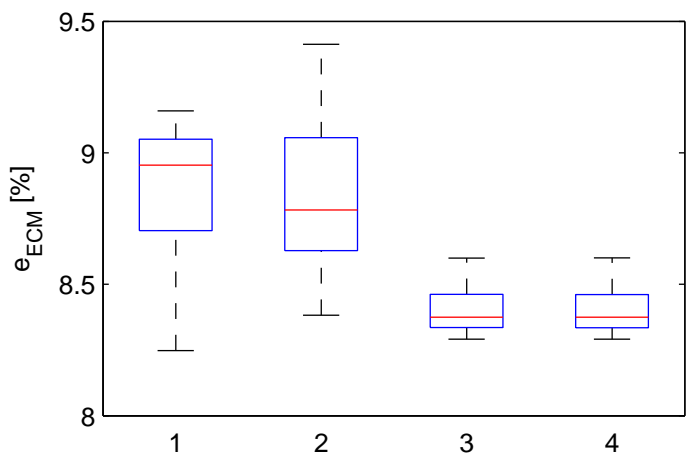

a.

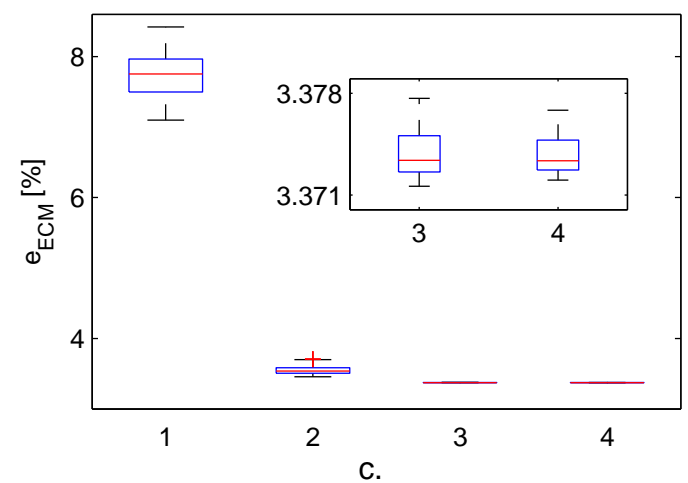

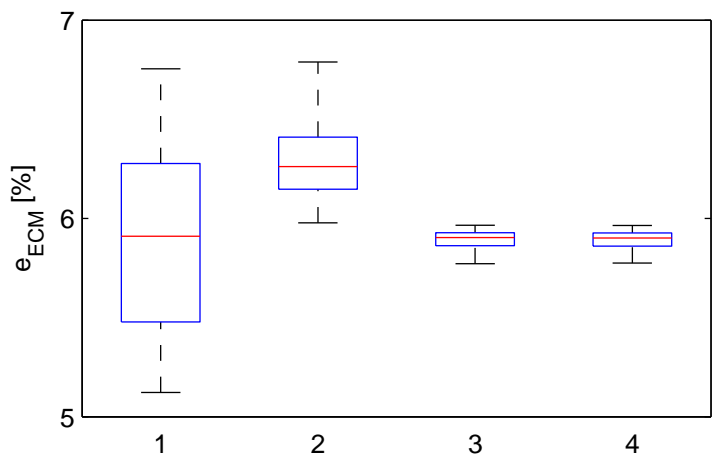

b.

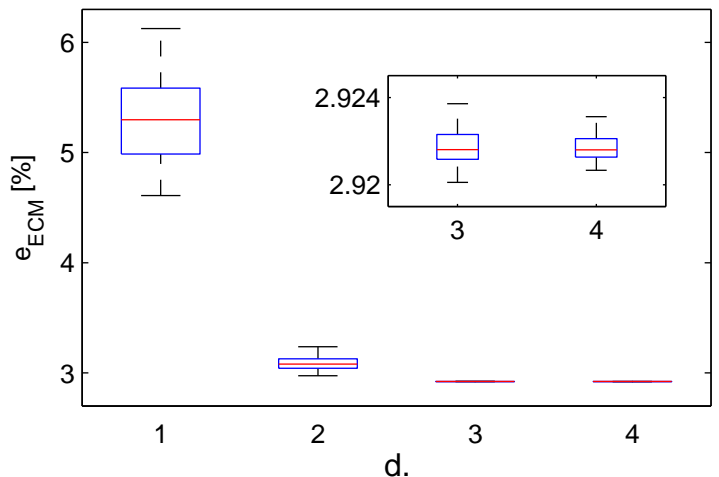

Figura 8.2: ECM normalizado porcentual $\left(e_{E C M}\right)$ considerando distinta cantidad de gradientes y esquemas. Se muestran los resultados correspondientes a los esquemas propuestos en 1: Poonawalla y Zhou (2004), 2: Skare et al. (2000), 3: Jones et al. (1999) y 4: Koay et al. (2011), considerando $N=6$ en a., $N=10$ en b., $N=30$ en c. y $N=40$ en d.

en donde puede observarse que las conclusiones efectuadas considerando el ECM son también válidas para el presente caso.

A pesar de que los esquemas 3 y 4 presentan similares cotas de desempeño, surge la recomendación de la utilización del esquema 4 tanto por su simplicidad como por su fácil adaptación al número de gradientes deseado, siendo el mismo el esquema elegido para el análisis posterior del capítulo. 


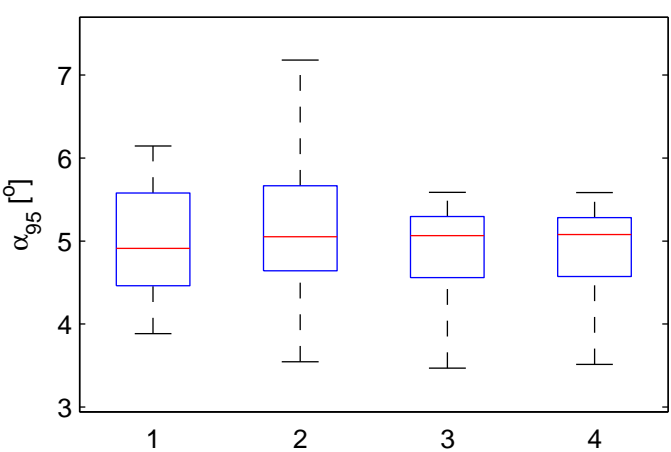

a.

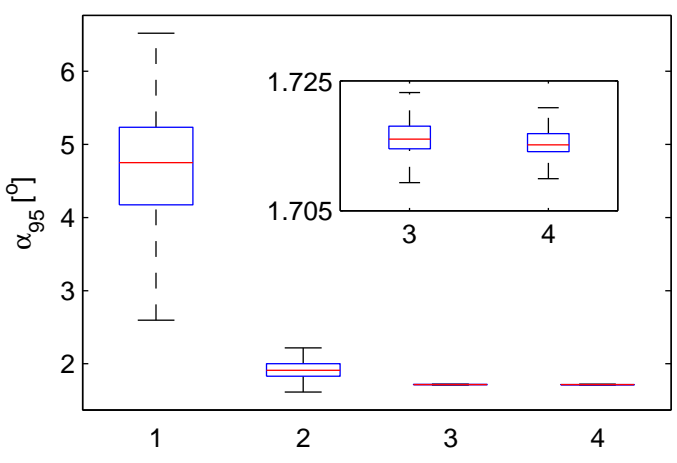

C.

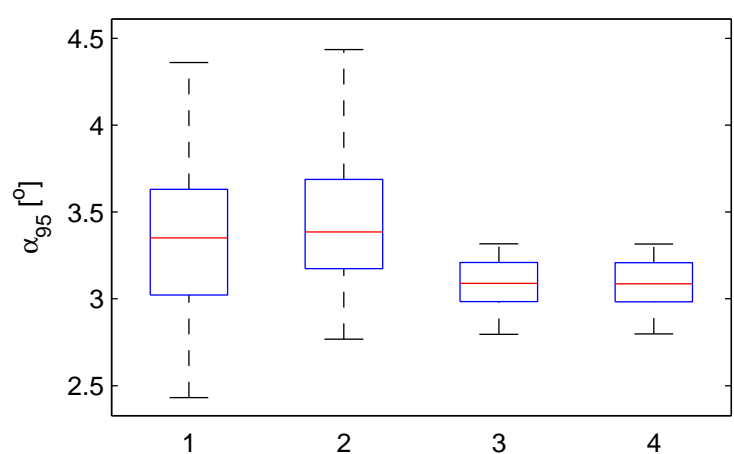

b.

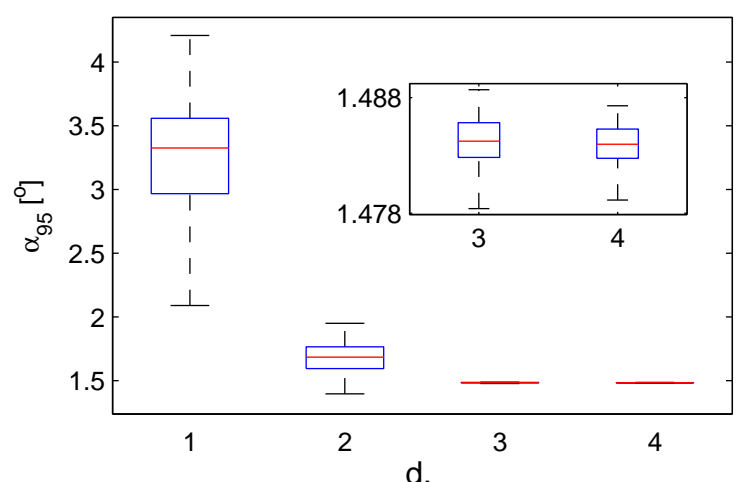

Figura 8.3: Ángulo de concentración de $95 \%$ considerando distinta cantidad de gradientes y esquemas. Se muestran los resultados correspondientes a los esquemas propuestos en 1: Poonawalla y Zhou (2004), 2: Skare et al. (2000), 3: Jones et al. (1999) y 4: Koay et al. (2011), considerando $N=6$ en a., $N=10$ en b., $N=30$ en c. y $N=40$ en d.

\section{Influencia de la forma del tensor}

Una vez caracterizados los esquemas de gradiente se evaluó la influencia de la forma del tensor en el error de la estimación. En la Figura 8.4 se muestra $e_{E C M}$ (a.) y $\alpha_{95}$ (b.) en función de la magnitud de los dos autovalores más pequeños del tensor. Para ello se supuso $\lambda_{1}=1 \times 10^{-3} \mathrm{~mm}^{2} / \mathrm{s}$ y $\lambda_{2}$ y $\lambda_{3}$ tomando valores en el intervalo $[0,1 ; 1] \times 10^{-3} \mathrm{~mm}^{2} / \mathrm{s}$. Se consideraron 6 direcciones de gradiente y una bobina adquisidora. Dada la pequeña varianza espacial del esquema seleccionado, en esta figura (al igual que en las restantes de la presente subsección) sólo se muestra el promedio de los resultados respecto de las direcciones del tensor sin indicar la desviación estándar. 


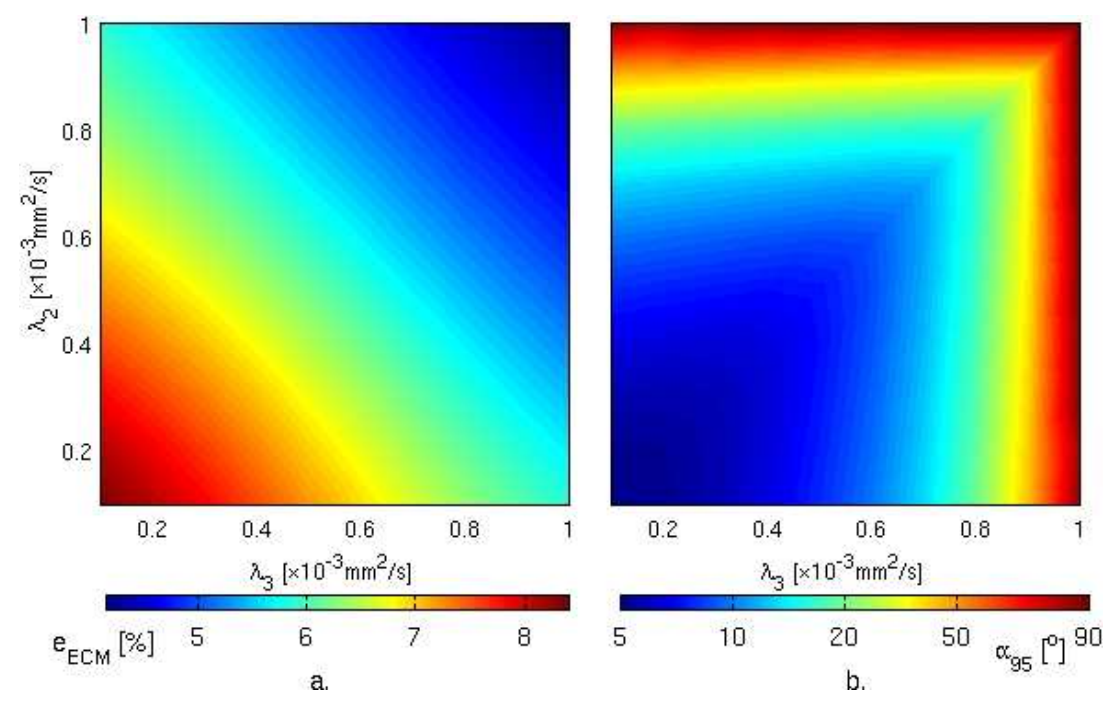

Figura 8.4: $e_{E C M}$ (a.) y ángulo de concentración de $95 \%$ (b.) en función de la forma del tensor, considerando $L=1$ y $N=6$.

Puede verse que el ECM normalizado es mayor para tensores del tipo fibra $\left(\lambda_{3}\right.$ y $\lambda_{2}$ pequeños) mientras que decrece a medida que los tensores presentan forma más esférica. Sin embargo, la influencia de la forma del tensor repercute en mayor proporción en la estimación del autovector principal. En la Figura 8.4b. se observa que el ángulo de concentración de $95 \%$ resulta pequeño (aproximadamente $5^{\circ}$ ) para tensores del tipo fibra, pero este crece notablemente para tensores con uno o ambos autovalores secundarios cercanos al autovalor principal. Esta situación corresponde a tensores del tipo disco y esfera, en donde el error en la determinación del autovalor principal radica en la igualdad de 2 ó 3 de ellos. Cabe destacar que estas relaciones se mantienen al aumentar el número de gradientes del esquema, reduciendo su amplitud en proporción similar a las mostradas por las Figuras 8.2 y 8.3.

El estudio de del coeficiente de variación en función de la forma del tensor permite evaluar y comparar de un modo explícito la conveniencia de los factores escalares cuya finalidad sea representar la anisotropía de dicho tensor. En la Figura 8.5 se muestran $e_{R A}$ (a.), $e_{F A}$ (b.) y $e_{E A R}$ (c.) en función de los dos menores autovalores del tensor. Las condiciones de simulación fueron las mismas a las de la Figura 8.4.

Es posible destacar diferencias entre ellos, como ser la reducción del coeficiente 


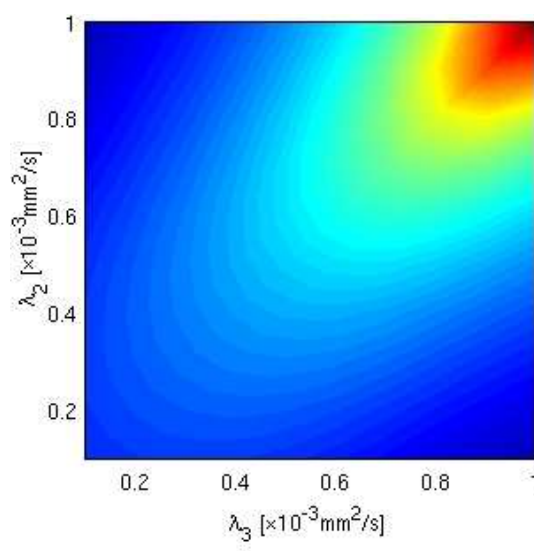

a.
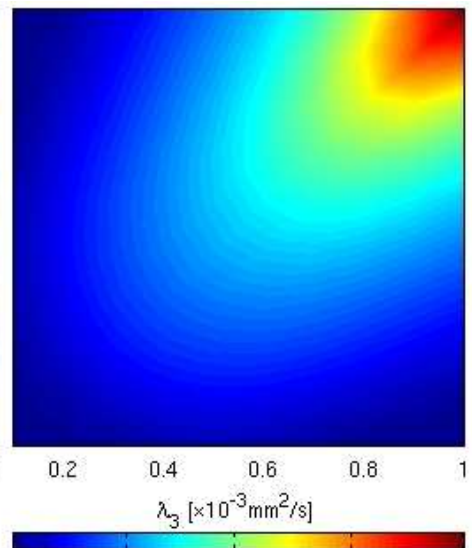

$\begin{array}{lllll}4 & 7 & 15 & 34 & 74\end{array}$ e [\%]

b.

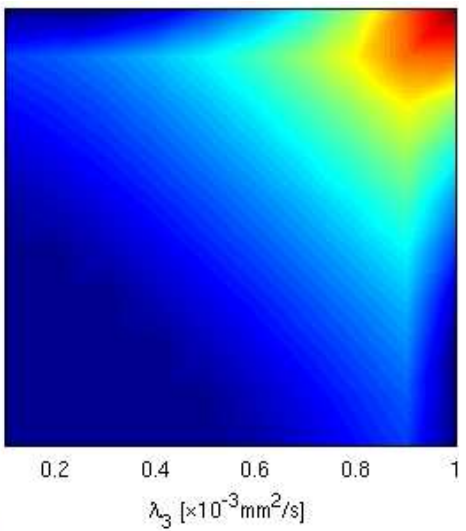

c.

Figura 8.5: Error en la estimación de parámetros escalares en función de la forma del tensor. Se muestran $e_{R A}$ (a.), $e_{F A}$ (b.) y $e_{E A R}$ (c.) en función de los dos menores autovalores del tensor de difusión, considerando $L=1$ y $N=6$.

de variación del FA respecto del RA en magnitud, manteniendo ambas similares variaciones respecto a la forma. Sin embargo, es aún más notable la diferencia del coeficiente de variación del EAR respecto al de los dos anteriores, principalmente por ser menor a éstos en zonas de tensores de tipo fibra. Sin embargo, para regiones en donde los tensores son del tipo disco, el EAR presenta coeficientes de variación mayores al correspondiente al FA e, incluso, al RA. Luego, la comparación anterior actúa como factor de mérito que permite decidir el parámetro a utilizar de acuerdo a la aplicación necesaria. Al igual que en el caso de la Figura 8.5, la distribución de los coeficientes de variación en función de la forma del tensor se mantienen en el caso de considerar mayor cantidad de gradientes de campo, reduciendo su amplitud del mismo modo que se aclaró previamente.

En las figuras anteriores se consideró una sola bobina adquisidora, lo cual genera el interrogante de la influencia de múltiples bobinas en la estimación de los parámetros y su variación con la forma del tensor. En el caso de suponer al ruido medido por las bobinas independiente e idénticamente distribuido (con distribución Rice bajo este supuesto) la mínima desviación estándar en la estimación (y consecuentemente el coeficiente de variación) considerando $L$ bobinas se reducirá $\sqrt{L}$ veces respecto de 
la utilización de una sola. Sin embargo, el modelo de ruido aquí utilizado contempla la correlación no nula entre señales medidas por diferentes bobinas. Luego, resulta de interés evaluar la validez de la aproximación de independencia y analizar la importancia del modelo propuesto. Para ello se plantea el cálculo de un coeficiente $\rho$ que permita evaluar esto de un modo sencillo. Sea $e_{A}(l)$ el coeficiente de variación porcentual del parámetro $A$ considerando $l$ bobinas adquisidoras. Luego, se define

$$
\rho_{A}=\left(\frac{\sum_{l=1}^{L}\left(e_{A}(1) / \sqrt{l}-e_{A}(l)\right)^{2}}{\sum_{l=1}^{L} e_{A}^{2}(l)}\right)^{1 / 2} \times 100,
$$

en donde $L$ es el total de bobinas utilizadas. Este parámetro permite evaluar la aproximación de independencia respecto de la forma del tensor. En la Figura 8.6 se muestra la variación de $\rho_{E C M}$ (a.), $\rho_{F A}$ (b.) y $\rho_{\alpha}$ (c.) en función de los dos autovalores más pequeños del tensor.

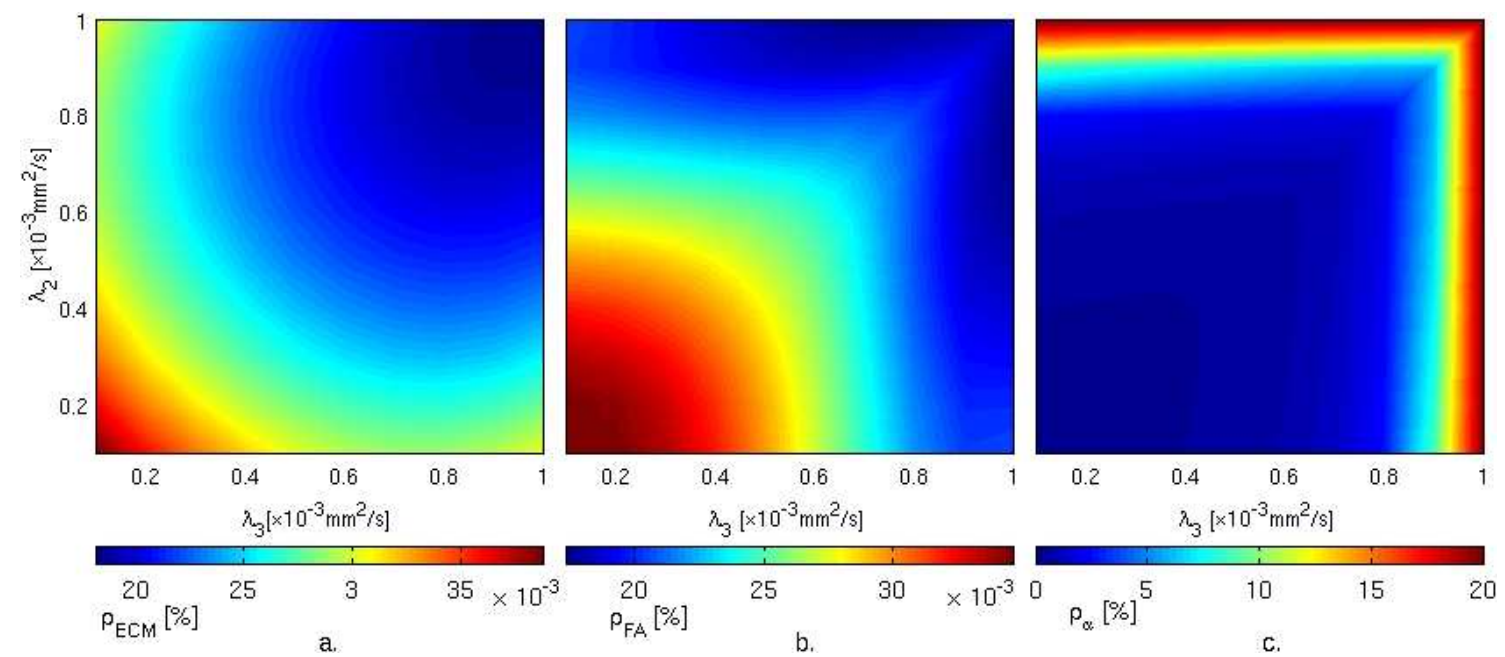

Figura 8.6: Variación de $\rho_{E C M}$ (a.), $\rho_{F A}$ (b.) y $\rho_{\alpha}$ (c.) en función de los dos autovalores más pequeños del tensor.

Se aprecia claramente que la aproximación resulta sumamente aceptable en el caso de considerar $e_{E C M}$ y $e_{F A}$, resultando ser $\rho$ aproximadamente $0.03 \%$ (lo mismo ocurre con $e_{R A}$ y $\left.e_{E A R}\right)$. No es igual en el caso de $\alpha_{95}$, resultando válida la aproximación para la gran mayoría de las formas, pero no todas. Luego, en caso de considerar 
$\lambda_{3} \leq \lambda_{2} \leq 0,85 \lambda_{1}$ la aproximación podrá suponerse válida $\left(\rho_{\alpha}<5 \%\right)$, no resultando igual para tensores de forma de disco o esfera. En conclusión, y salvo el caso recién mencionado, considerar independencia en la adquisición de las bobinas resulta ser una aproximación mayormente válida, por lo que en lo siguiente se simularán (a menos que se indique lo contrario) sólo disposiciones con una bobina receptora, debiendo dividir los resultados por $\sqrt{L}$ en el caso de considerar $L$ de éstas.

\section{Influencia de la SNR y el factor $b$}

Por último (en lo que respecta a datos sintéticos) se procedió a evaluar la influencia de la SNR y el factor $b$. En las Figuras 8.7 y 8.8 se muestran, respectivamente, resultados correspondientes a las variaciones de $e_{E C M}$ y $\alpha_{95}$ respecto del número de gradientes de campo considerado y los factores $L$ (a.) (solo por interés comparativo), $b$ (b.) y $S N R$ (c.). Cuando no se supusieron variables se consideraron $L=1$, $b=1200 \mathrm{~s} / \mathrm{mm}^{2}$ y $S N R=30$. Dado que la difusión Gaussiana puede ser asumida solo si $b<3 \times 10^{3} \mathrm{~s} / \mathrm{mm}^{2}$ (Assaf y Basser, 2005), se consideraron valores de $b$ en el intervalo $[400,2500] \mathrm{s} / \mathrm{mm}^{2}$.
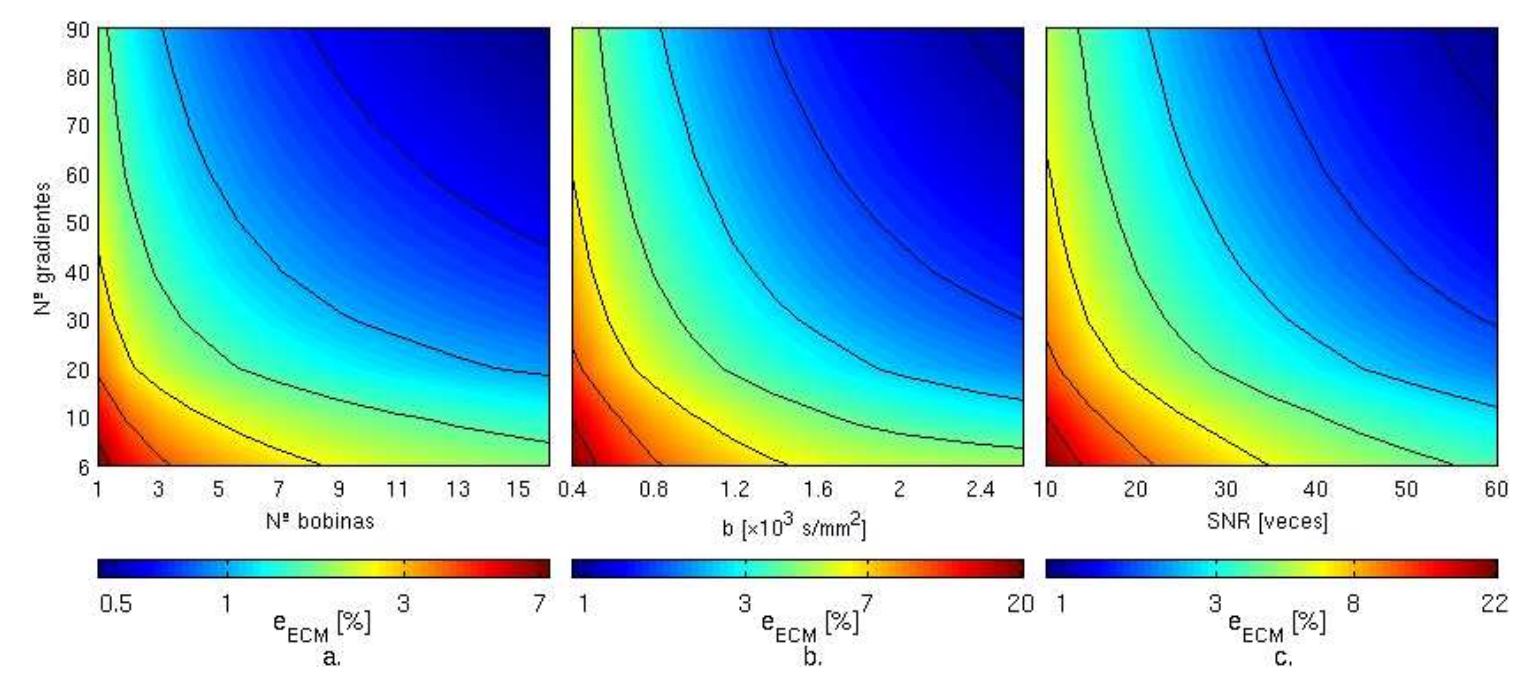

Figura 8.7: Variación del $e_{E C M}$ respecto al número de gradientes de campo aplicado y el número de bobinas adquisidoras (a.), el factor $b$ (b.) y la SNR (c.).

Se desprende de estas figuras la caracterización del ruido en función de los 

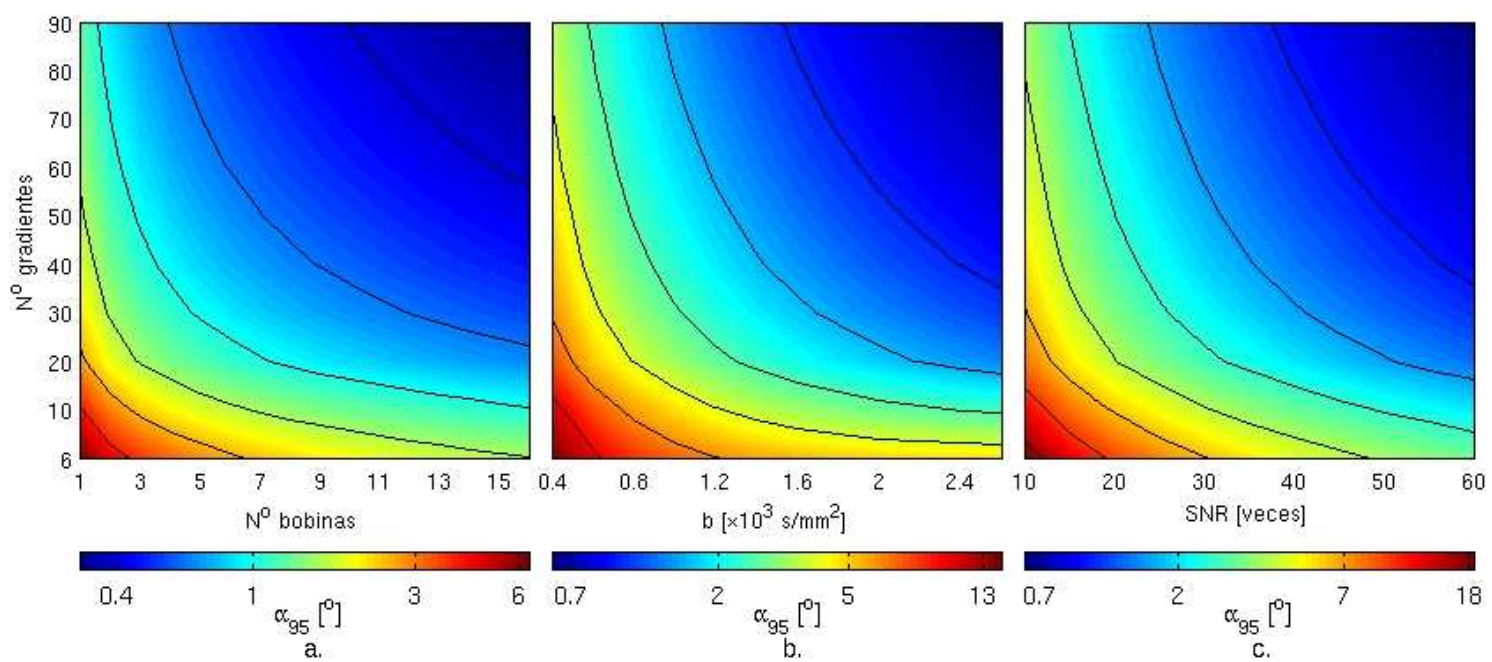

0.7
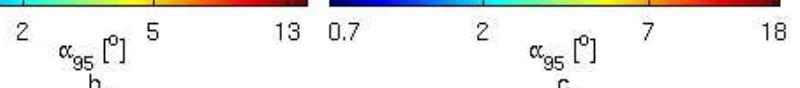

Figura 8.8: Variación del $\alpha_{95}$ respecto al número de gradientes de campo aplicado y el número de bobinas adquisidoras (a.), el factor $b$ (b.) y la SNR (c.).

parámetros del modelo. Puede observarse que si bien la SNR no es definida por el usuario, sus efectos pueden paliarse mediante la incorporación de bobinas adquisidoras y/o el aumento del número de gradientes y del factor $b$. Es preciso aclarar que la influencia de la SNR y el factor $b$ resulta ser prácticamente constante frente a cambios de $N$, variando estas constantes de acuerdo a las Figuras 8.2 y 8.3.

\subsubsection{Resultados utilizando datos reales}

Ya caracterizado el límite teórico del error en la estimación utilizando datos sintéticos se procedió a calcular dichos límites considerando datos reales. Esto permite realizar un análisis en donde las verdaderas orientaciones y formas de los tensores son tenidas en cuenta en el momento de establecer las cotas de desempeño. Para ello se utilizó el atlas IIT2 ${ }^{1}$ (Zhang et al., 2011), el cual corresponde al promedio de 67 sujetos normalizados al atlas ICBM-152 y cuyos detalles se remiten a la mencionada publicación. Primero se muestran resultados globales considerando la totalidad de los datos del volumen, de los cuales es posible extraer información probabilística de sumo interés. Luego, se enseñan resultados sobre un corte axial que, a modo de

\footnotetext{
${ }^{1}$ Datos disponibles en http://www.iit.edu/ mri
} 
ejemplo, permite evaluar la distribución espacial de los errores en la estimación del tensor de difusión y funciones de éste.

\section{Resultados sobre el total del volumen}

Se consideraron la totalidad de los datos del atlas para comparar la influencia del error en los parámetros escalares derivados del tensor. Esto permite evaluar tanto el desempeño de los factores generalmente utilizados así como su comparación con parámetros escalares alternativos de uso menos difundido. En la Figura 8.9 se muestra $e_{R A}, e_{F A}$ y $e_{E A R}$ en función del FA y considerando $N=6$ (a.) y $N=30$ (b.). Puede observarse que para tensores con FA mayor a 0,3 la mínima
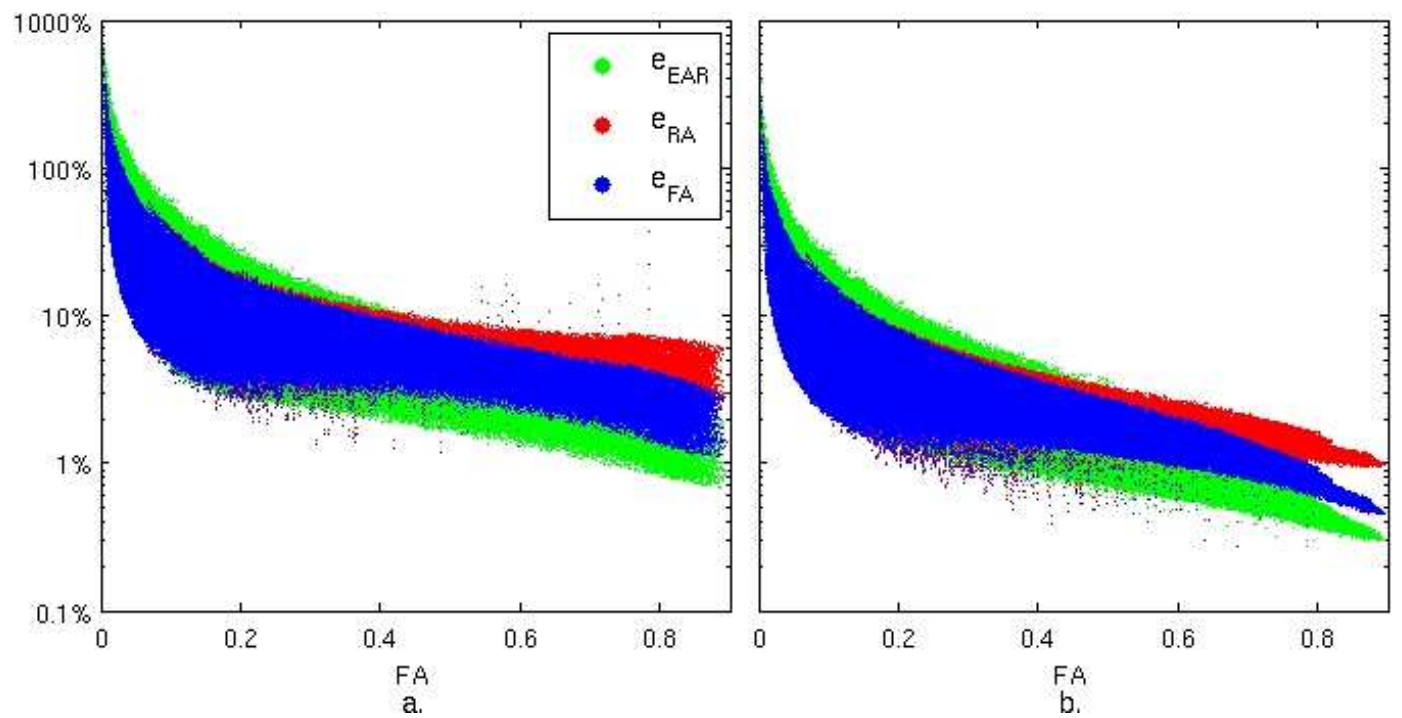

Figura 8.9: $e_{R A}, e_{F A}$ y $e_{E A R}$ sobre la totalidad de voxels del volumen de la RM en función del FA y considerando $N=6$ (a.) y $N=30$ (b.).

desviación estándar en la estimación del EAR es menor que en el caso del FA y esta a su vez es menor que la correspondiente al RA. Sin embargo, esta relación no se mantiene para tensores con FA menor a 0,3, en donde la mínima desviación estándar en la estimación del EAR es mayor que la correspondiente a los otros dos factores escalares bajo estudio. Esto denota la relación no lineal entre el FA y el EAR tal como se mostró previamente en la Figura 8.5. 
En segundo lugar se evaluó la reconstrucción del autovector principal sobre los datos reales del atlas. Tal como se mencionó anteriormente, el ángulo de confianza de $95 \%$ depende de la forma y orientación del tensor de difusión, pudiendo ser este último aspecto despreciable si se utilizan esquemas de gradiente de varianza espacial pequeña (ver Sección 8.3.1). De este modo, los errores en la adquisición de TD sobre un volumen completo dependerán de la distribución de tensores y sus respectivas formas. Luego, resulta factible el cálculo de la densidad de probabilidad (empírica) de $\alpha_{95}$ en el volumen, $f_{\alpha_{95}}\left(\alpha_{95}\right)$, la cual permite cuantificar la incidencia del ruido en la mínima desviación estándar del estimador del ángulo del autovector principal en función del número de gradientes de campo y bobinas adquisidoras utilizadas.

En la Figura 8.10 se muestra $f_{\alpha_{95}}\left(\alpha_{95}\right)$ considerando $L=1$ y $N$ variable (a.), y $N=30$ y $L$ variable (b.). Para ello sólo se tuvieron en cuenta los tensores con FA mayor a 0,2, dado que son los únicos de interés en estudios de reconstrucción de tractos fibrilares (Taoka et al., 2009). Se vislumbran claramente las mejoras en la
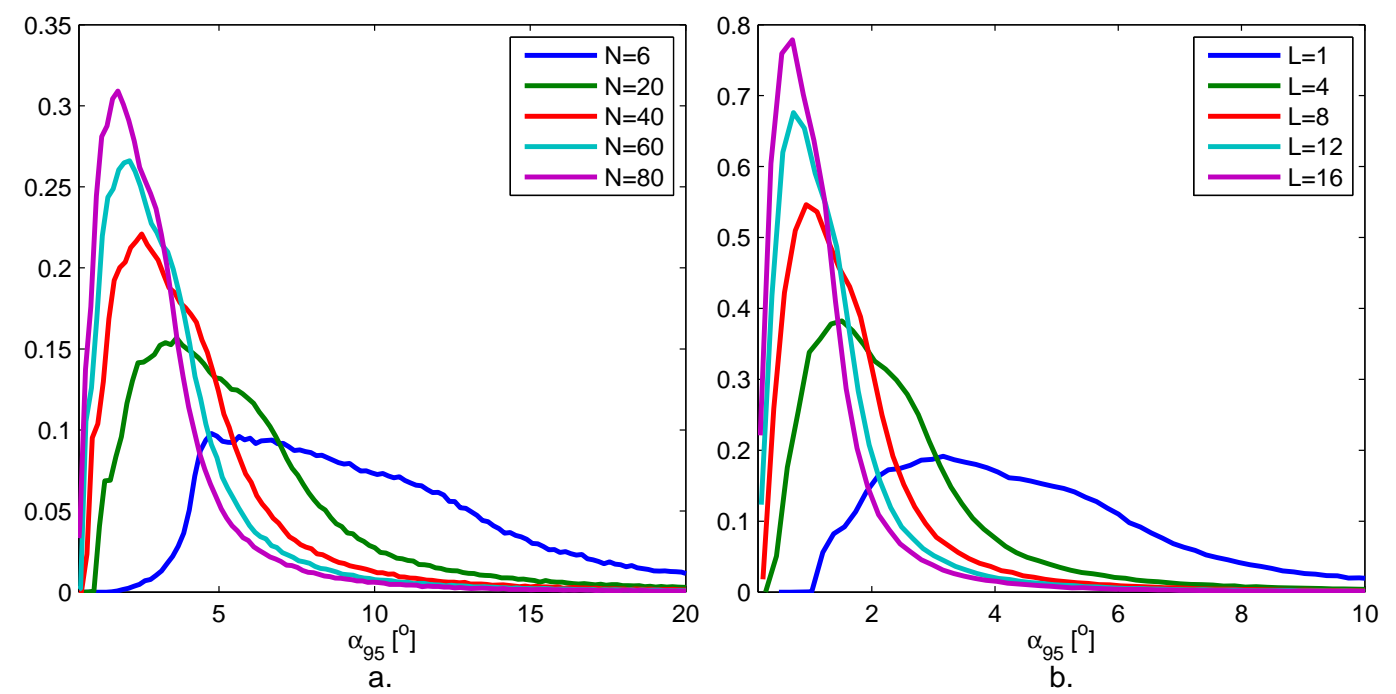

Figura 8.10: $f_{\alpha_{95}}\left(\alpha_{95}\right)$ considerando $L=1$ y $N$ variable (a.) y $N=30$ y $L$ variable (b.).

estimación del autovector principal a medida que aumenta el número de gradientes y, especialmente, el de bobinas adquisidoras.

En base al cálculo de $f_{\alpha_{95}}\left(\alpha_{95}\right)$ es posible determinar el límite de $95 \%$ de probabilidad de $\alpha_{95}$ sobre el total de voxels de interés en el volumen. Este límite, definido 
como

$$
\beta_{95}: \int_{0}^{\beta_{95}} f_{\alpha_{95}}\left(\alpha_{95}\right) d \alpha_{95}=0,95,
$$

permite afirmar que el $95 \%$ de los autovectores del volumen bajo estudio pueden estimarse con un valor de $\alpha_{95}$ menor a $\beta_{95}$. Dado que $f_{\alpha_{95}}\left(\alpha_{95}\right)$ es función de $L$ y $N$, el cálculo de $\beta_{95}$ permite evaluar la necesidad de la incorporación de bobinas adquisidoras y/o gradientes de campo de acuerdo al error que se pretenda del estudio.

En la Figura 8.11 se muestra $\beta_{95}$ en función del número de gradientes de campo considerando $1,4,8,12$ y 16 bobinas adquisidoras, diferenciadas con distintos marcadores. Resulta evidente que las cotas teóricas en este caso se ven sumamente

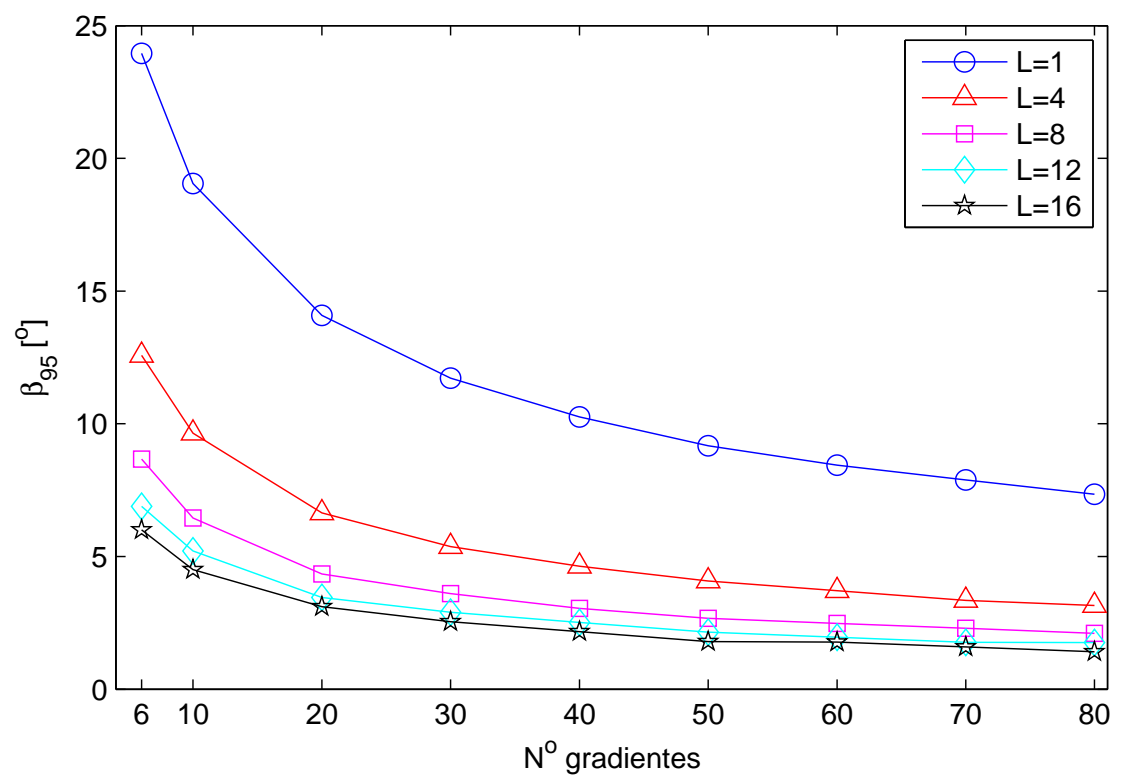

Figura 8.11: $\beta_{95}$ en función del número de gradientes de campo considerando 1, 4, 8, 12 y 16 bobinas adquisidoras, diferenciadas con distintos marcadores.

disminuidas tanto con la incorporación de bobinas como con el aumento de gradientes del esquema. Esto puede evidenciarse, por ejemplo, en el hecho de que $\beta_{95}$ es menor en el caso de considerar $N=10$ y $L=8$ que utilizando un esquema de 80 gradientes pero una sola bobina adquisidora. Estos resultados avalan la posibilidad de considerar mayor número de bobinas adquisidoras con la finalidad de reducir el número de gradientes $\mathrm{y}$, de este modo, el tiempo de adquisición, sin sacrificar en 
ninguna proporción los resultados de la estimación del autovector principal.

\section{Resultados sobre un corte axial}

Finalmente, se muestran resultados sobre un corte axial $(Z=90$ en las coordenadas estándar de MNI). Esto permite evaluar espacialmente las ventajas en la consideración de sistemas de múltiples bobinas adquisidoras y mayor número de gradientes de campo. Para ello se consideraron sólo tensores con $F A \geq 0,1$. El fondo de las imágenes corresponde al mismo corte de la resonancia T2 provista por el atlas.

En la Figura 8.12 se enseñan los coeficientes $e_{R A}$ (izquierda), $e_{F A}$ (centro) y $e_{E A R}$ (derecha) considerando $N=6$ (arriba) y $N=40$ (abajo). Puede verse que los

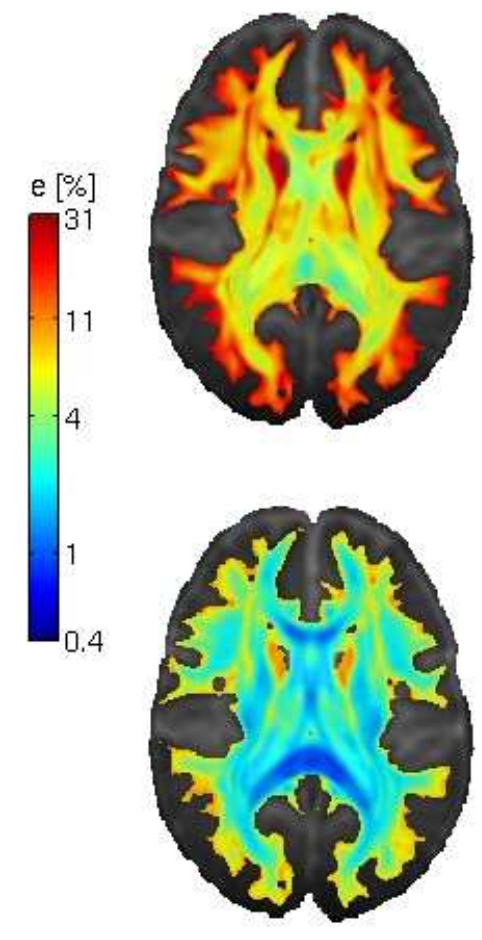

$\mathrm{RA}$
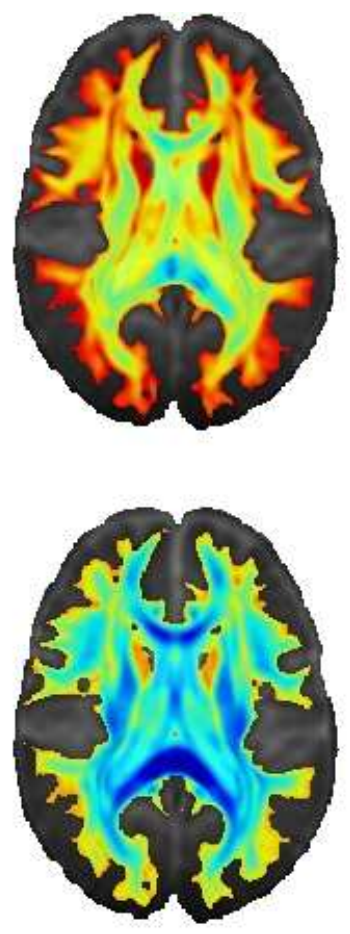

FA
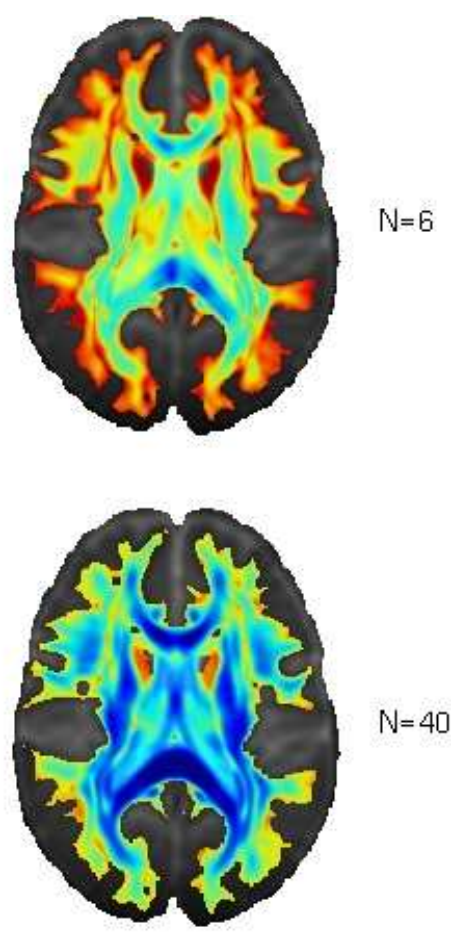

EAR

Figura 8.12: Corte axial $Z=90$ mostrando $e_{R A}$ (izquierda), $e_{F A}$ (centro) y $e_{E A R}$ (derecha) considerando $N=6$ (arriba) y $N=40$ (abajo).

coeficientes de variación son sensibles a la región del cerebro bajo análisis pues, como se mostró en anteriormente, dichos coeficientes dependen de la forma del tensor. Para 
realizar un análisis más completo de la situación en la Figura 8.13 se muestran los coeficientes $C_{l}, C_{e}$ y $C_{d}$ definidos por (Westin et al., 1997)

$$
C_{l}=\frac{\lambda_{1}-\lambda_{2}}{\lambda_{1}+\lambda_{2}+\lambda_{3}}, C_{e}=\frac{3 \lambda_{3}}{\lambda_{1}+\lambda_{2}+\lambda_{3}}, C_{d}=\frac{2\left(\lambda_{2}-\lambda_{3}\right)}{\lambda_{1}+\lambda_{2}+\lambda_{3}},
$$

los cuales permiten diferenciar tensores tipo fibra (alto $C_{l}$ ), esfera (alto $C_{e}$ ) y disco $\left(\right.$ alto $\left.C_{d}\right)$ entre sí.
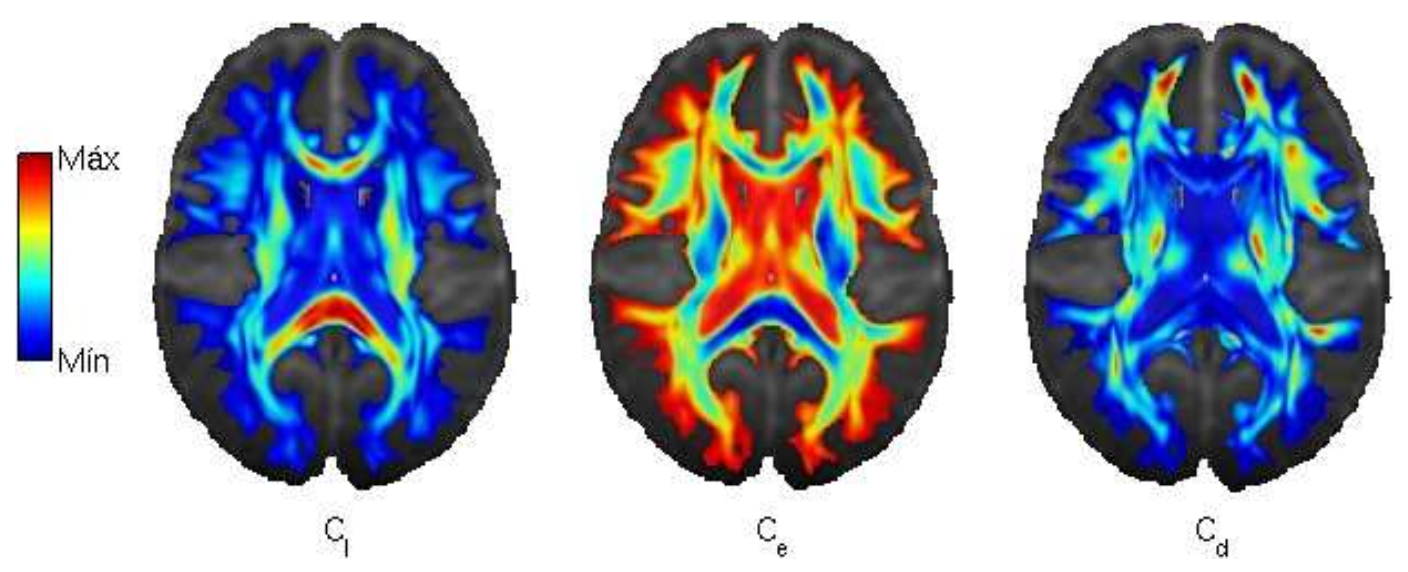

Figura 8.13: Corte axial $Z=90$ mostrando $C_{l}$ (izquierda), $C_{e}$ (centro) y $C_{d}$ (derecha).

A partir de ello resulta sencillo observar, mediante una comparación directa entre ambas figuras, que los coeficientes de variación correspondientes a los tres parámetros son mayores en zonas de $C_{e}$ relativamente alto, sobre todo en las inmediaciones de los ventrículos y en la interfaz entre materia blanca y gris. Lo mismo ocurre en zonas de tensores de tipo disco, aunque en menor proporción. Asimismo, de la Figura 8.12 se desprende que la estimación del EAR resulta recomendable en regiones de alto $C_{l}$, pero no así en regiones de predominancia de tensores del tipo disco y, sobre todo, esféricos. Esto permite reafirmar la idea desarrollada anteriormente, mediante la que el EAR resulta un parámetro recomendable para utilizar como umbral en estudios de tractografía, obteniendo menor coeficiente de variación en zonas fibrosas en comparación al $R A$ y el $F A$. Cabe destacar que, tal como se encontró en la subsección anterior, la utilización de $L$ bobinas receptoras permitirá obtener coeficientes de variación $\sqrt{L}$ veces más pequeños.

Del mismo modo se calculó $e_{E C M}$ considerando $\mathrm{N}=6,20$ y 50, y sus resultados se muestran en la Figura 8.14. En este caso puede verse que el coeficiente de variación 

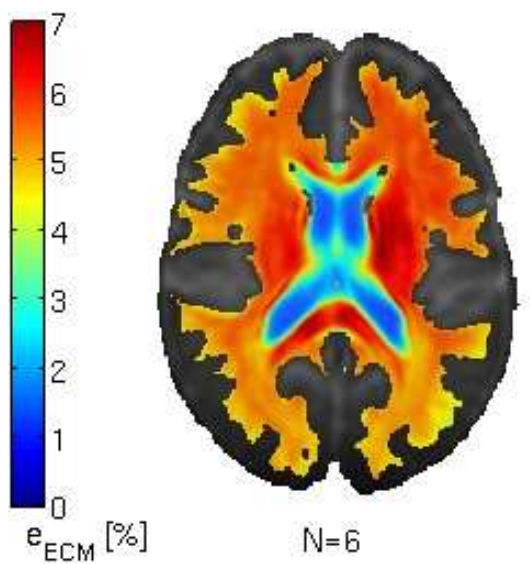

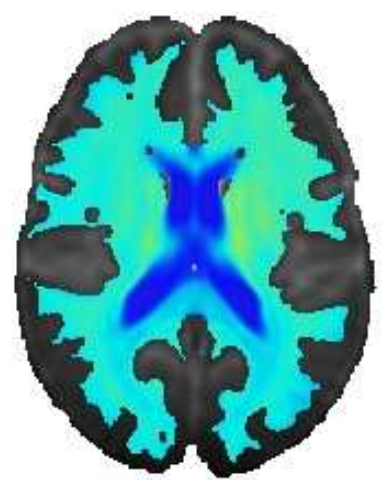

$N=20$

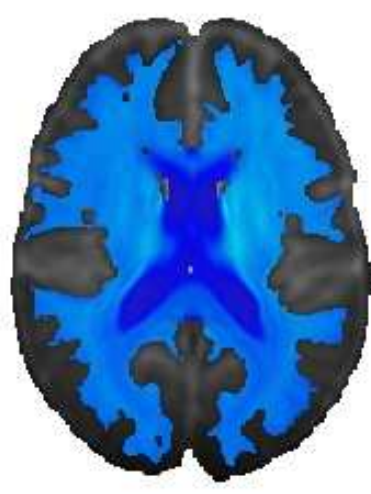

$\mathrm{N}=50$

Figura 8.14: Corte axial $Z=90$ mostrando $e_{E C M}$ y considerando $N=6$ (izquierda), $N=20$ (centro) y $N=50$ (derecha).

es más homogéneo que en el caso de los coeficientes escalares, tal como se mostró en la Figura 8.4a.

Finalmente se calculó $\alpha_{95}$ considerando $N=6,20$ y 50 y una bobina adquisidora, mostrando los resultados en la Figura 8.15. Se observa que el ángulo de confianza
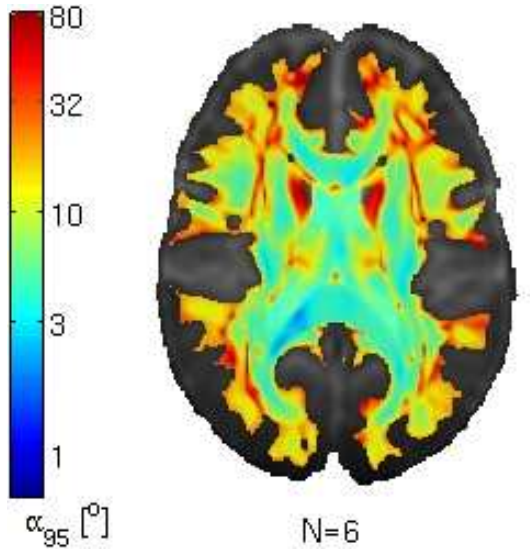

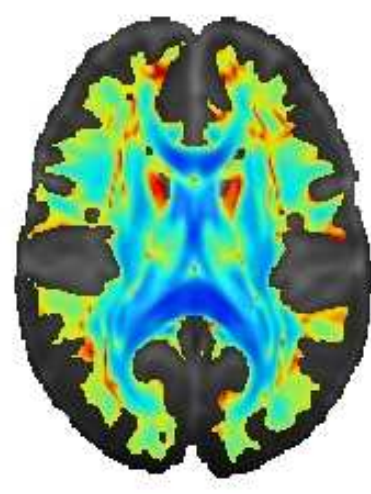

$N=20$

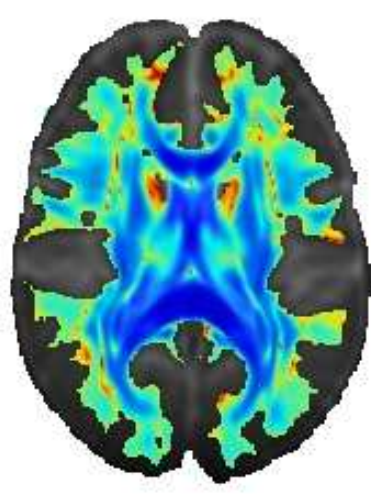

$\mathrm{N}=50$

Figura 8.15: Corte axial $Z=90$ mostrando $\alpha_{95}$ y considerando $N=6$ (izquierda), $N=20$ (centro) y $N=50$ (derecha).

de $95 \%$ del máximo autovector disminuye notablemente al aumentar el número de gradientes de campo utilizados, sobre todo en regiones en donde $C_{l}$ es alto (las cuales, en definitiva, serán las de interés para estudios de tractografía). En este caso también se cumple la reducción del ángulo en $\sqrt{L}$ veces al considerar $L$ bobinas 
receptoras, aunque dicha relación es válida sólo para tensores que no sean ni discos ni esferas (ver Figura 8.4b.). La relación entre el número de bobinas y $\alpha_{95}$ es menor a medida que aumentan $C_{e} \mathrm{y}$, sobre todo, $C_{d}$, por lo que se debe tener especial cuidado en estos casos.

\subsection{Discusión y Conclusiones}

En el presente capítulo se estudió la influencia del ruido en la estimación del TD y factores derivados de éste. Con la finalidad de contemplar la totalidad de las variables de interés se presentó un modelo de señal de RM-TD que permitió considerar la incorporación del número de bobinas receptoras y su repercusión en la posterior estimación. Una vez caracterizada la señal se propuso la utilización de la CRB como entorno de análisis para cuantificar los desempeños óptimos en la estimación del tensor en función de parámetros de interés, como lo son el número de gradientes de campo, la cantidad de bobinas adquisidoras utilizadas, la forma de los tensores y la SNR.

El entorno aquí planteado difiere de los comúnmente propuestos en la literatura con el fin de caracterizar el error en la estimación; del método de propagación de errores (considerado en Poonawalla y Zhou (2004); Chang et al. (2007); Hasan (2007); Koay et al. (2007)) en la incorporación de información sobre la distribución del ruido de fondo, y del método no paramétrico de bootstrap (estudiado en Jones (2003); Pajevic y Basser (2003); Hasan et al. (2004); O'Gorman y Jones (2006)) en la simplicidad de su realización (sin ser para ello necesarias múltiples mediciones) y obtención de fórmulas paramétricas que permiten un adecuado análisis teórico. Sin embargo, su mayor fortaleza reside en el estudio de la influencia de los errores independientemente del algoritmo de estimación utilizado, resultando en cotas de desempeño óptimo válidas para cualquier método de estimación insesgado.

En primer lugar se aplicaron los resultados en el análisis comparativo entre diversos esquemas de gradientes propuestos en la bibliografía. Basados en el mínimo ECM y el mínimo ángulo de concentración de 95\% se concluyó que los esquemas propuestos por Jones et al. (1999) y Koay et al. (2011) ofrecen tanto menor error en 
la reconstrucción como también menor varianza espacial, resultando ser los aquí recomendados. Estas conclusiones concuerdan con trabajos existentes (Jones, 2004; Stirnberg et al., 2009; Mang et al., 2009), en donde el esquema planteado por Jones et al. (1999) resulta ser sugerido. Asimismo, el notable desempeño encontrado al utilizar el esquema de Koay et al. (2011) permite validar las hipótesis existentes sobre la conveniencia del uso de esquemas lo más uniformemente distribuidos sobre la superficie esférica (Mukherjee et al., 2008).

Luego se presentaron resultados de la estimación en función de la forma del tensor. Se encontró que el ECM normalizado en la estimación de $\boldsymbol{D}$ aumenta a medida que el tensor toma forma de fibra. Sin embargo, el error en el mínimo ángulo de confianza de $95 \%$ resultó ser pequeño para tensores de este tipo, aumentando a medida que toma forma de disco o esfera. Esta caracterización resulta de suma utilidad, pues permite evidenciar que el error en la estimación del autovector principal no es lineal respecto del FA, el cual es generalmente utilizado como umbral en estudios de tractografía.

La misma metodología se utilizó a fin de comparar los parámetros escalares RA, FA y EAR utilizados con el objeto de cuantificar la anisotropía de los tensores. Se encontró que el FA se puede estimar mejor que el RA considerando cualquier forma del tensor, resultando ser el coeficiente de variación de dichos parámetros escalares inversamente proporcional a su valor. Estos resultados coinciden con los de Papadakis et al. (1999), en donde se evalúan sólo tres formas de tensores. Sin embargo, se mostró que el EAR obtiene aún mejor desempeño que el FA en zonas de tensores del tipo fibra, no ocurriendo lo mismo con la totalidad de los tensores del tipo disco. Esto concuerda con trabajos previos en donde se muestra que el EAR logra mejores desempeños que los índices escalares tradicionales en términos de sensibilidad (Afzali y Soltanian-Zadeh, 2010; Hayes et al., 2011), especialmente en anormalidades profundas de la materia blanca y en la caracterización pericortical de las estructuras de dicho tejido (Kang et al., 2010). Este hecho conlleva al estudio del EAR como posible factor de umbral para la realización de tractografía.

Con el fin de analizar la influencia de la incorporación de bobinas al sistema receptor se estudió la validez de la consideración de independencia de las bobinas 
respecto del ruido de fondo. Tras el análisis se logró validar dicha aproximación para la estimación de la totalidad de los parámetros escalares derivados del tensor de difusión, con la excepción del ángulo de confianza de 95\%. Sin embargo, la aproximación para este último resultó ser válida para tensores de $C_{l}$ medio y alto, por lo que pocos tensores se verían exentos a la aproximación de independencia. El resultado de mayor interés al respecto es la disminución del coeficiente de variación de los parámetros estudiados y del ángulo de concentración de $95 \%$ en $\sqrt{L}$ veces en el caso de utilizar $L$ bobinas receptoras.

El entorno probabilístico planteado en el presente trabajo permitirá estudiar con notable rigor cotas de desempeño en la reconstrucción fibrilar, lo cual constituye un punto de inflexión en el grado de credibilidad de los métodos existentes. A diferencia de algunos trabajos (Anderson, 1990; Lazar y Alexander, 2003) el método aquí desarrollado permite considerar adecuadamente la distribución del ruido de adquisición, el cual puede ser muy influyente. Se plantea como trabajo futuro la consideración del ángulo de concentración de $95 \%$ aquí expuesto con el fin de analizar errores ineludibles en la tractografía y optimizar los parámetros de adquisición con el fin de reducir los errores a su mínima expresión.

Debe notarse que en el estudio realizado se consideró un modelo de tensor simple, el cual es por lejos el más utilizado, pero no el que mejor puede representar la situación. Extensiones al uso de modelos de tensores duales (Maier et al., 2001, 2004) o más complejos aún (como ser el modelo CHARMED propuesto en Assaf y Basser (2005)) resultan evidentes. Éstos, junto con el análisis de la influencia de la incorporación de la sensibilidad de las bobinas receptoras, serán objeto de futuros estudios. Cabe destacar que el presente análisis puede aplicarse a nuevas técnicas basadas en mediciones de RM, como las imágenes de difusión de curtosis (DKI), que permite describir el comportamiento no Gaussiano de la difusión en tejidos (Poot et al., 2010). 


\section{Capítulo 9}

\section{Conclusiones}

En esta tesis se presentaron distintos aportes relacionados con la electro y magnetoencefalografía, referidos tanto al problema directo como al problema inverso. Asimismo, se presentaron contribuciones en el campo de la resonancia magnética por tensor de difusión y la caracterización de los errores en su estimación.

Se desarrolló el método de los elementos finitos utilizando funciones de base lineales para la resolución del problema directo en E/MEG. Se mostraron sus ventajas tanto frente a otros métodos de resolución, como frente a formulaciones diferentes del mismo método. La simplicidad en su implementación y la versatilidad que provee respecto a la adaptabilidad del modelo permitió su aplicación en diferentes problemas ligados a E/MEG.

Se analizó la influencia del modelo utilizado en estudios en donde no se posee información suficiente para realizar modelos individuales. Para ello se desarrolló un modelo realista basado en atlas disponibles, comparando los resultados con el muy utilizado modelo esférico de tres capas. Utilizando señales reales de potenciales evocados se mostró que la adecuada selección del modelo puede resultar determinante en la localización de la fuente de actividad cerebral.

Se estudió el efecto de la adecuada consideración del modelo en estudios simultáneos de ECoG y E/MEG. Los resultados permitieron concluir en la influencia determinante de la grilla no conductora en dichos estudios, principalmente en EEG. Asimismo, se mostraron las ventajas de MEG frente a EEG en dichas circunstancias. 
Se analizó el efecto de las variaciones de pequeña amplitud en el posicionamiento de los electrodos de EEG, mediante un estudio de perturbaciones de primer orden a la solución del problema directo. Dichas variaciones de pequeña amplitud representan errores en la adquisición de la posición propios de los sistemas utilizados a tal fin. Los resultados mostraron que el error que causan las perturbaciones a la posición de los sensores en el problema inverso es, para los parámetros de posición de la fuente, del mismo orden que el ruido electrónico, resultando ser de segundo orden frente a fuentes de ruido tales como la actividad cerebral de fondo.

Se desarrolló un método de estimación sesgada por constricción de la matriz de covarianza espacio-temporal del ruido de fondo en EEG para situaciones en las que el número de realizaciones disponibles es escaso. Se mostraron las ventajas teórico-prácticas en el conocimiento a priori de los modelos existentes de dichas matrices, permitiendo lograr mejor desempeño que los estimadores generalmente utilizados. Dichas ventajas se mostraron sobre datos sintéticos y reales, manifestando la factibilidad y conveniencia del método.

Se estudió el desempeño de los estimadores por constricción de la matriz de covarianza de la actividad cerebral de fondo en la resolución del problema inverso en EEG utilizando beamforming. Se adaptó un método existente a mediciones de modo activo solamente, reduciendo las limitaciones que presenta su implementación. Se mostraron ventajas de los modelos de actividad cerebral disponibles, permitiendo la adecuada estimación a partir del conocimiento de contadas realizaciones de señal, propio de estudios relacionados a epilepsia.

Por último se analizó el error cometido en la adquisición de señales de RM-TD. Utilizando un marco teórico adecuado se obtuvieron expresiones que permitieron establecer los límites en el desempeño esperado considerando sistemas de una y múltiples bobinas adquisidoras. Estos últimos mostraron grandes ventajas en la estimación del mapa tensorial de difusión, pudiendo resultar determinante en la adecuada reconstrucción fibrilar del cerebro.

Existen muchas líneas de investigación que pueden continuarse más allá de los resultados mostrados en esta tesis, así como resultados parciales que requieren mayor confirmación teórica y experimental. 
En relación a los modelos de cabeza y la influencia de los dispositivos de medición intracraneales en E/MEG surgen varias líneas de trabajo. En principio, el método propuesto y las conclusiones a las cuales se arribaron impulsan hacia la planificación adecuada de un análisis teórico-experimental que permita establecer el grado de lesión visible con E/MEG tradicional, estableciendo los límites de las técnicas no invasivas. Asimismo, el método desarrollado puede aplicarse en el diseño de instrumentos de medición invasivos igual de efectivos que permitan una adecuada complementariedad con estudios simultáneos de E/MEG, tal como podría ser una grilla de ECoG con huecos entre los electrodos.

Dado que el modelo dipolar presenta múltiples limitaciones, se plantea la adaptación del método de resolución propuesto utilizando modelos de fuentes de actividad cerebral volumétricamente distribuidas. Se espera que éstas permitan una mejor representación de la fuente de actividad, conllevando a resultados más realistas.

Respecto de los estimadores por constricción de la matriz de covarianza, y tal como se expresara oportunamente, resulta deseable la adaptación de los mismos frente a variaciones de la actividad cerebral de fondo en sus distintas bandas de frecuencia. Esto implicaría un análisis de la validez de los modelos existentes y su adaptabilidad a dichas fluctuaciones temporales. Asimismo, resta la utilización y aplicación del método de beamforming por constricción utilizando señales de E/MEG reales.

Finalmente, y en relación al estudio de la estimación del mapa tensorial de difusión cerebral, existen muchas líneas de trabajo futuro. En principio, se propone el uso de la técnica desarrollada con el fin de obtener cotas en el desempeño de la reconstrucción de tractos fibrilares. Esto permitirá establecer límites teóricos sobre los métodos existentes y evaluar las ventajas propias de los sistemas de adquisición de múltiples bobinas. Asimismo, los resultados presentados en dicha materia permiten calcular cotas en la estimación del tensor de conductividad eléctrica, los cuales pueden ser utilizados en pos de evaluar su consecuente aplicación en la resolución del problema directo en E/MEG y los errores que involucra. 


\section{Apéndice A}

\section{Deducción de algunas fórmulas del Capítulo 2}

\section{A.1. Demostración del Teorema 2.3.1}

Sea $\boldsymbol{J}^{p}$ cuadrado integrable. Aplicando el valor absoluto a la forma lineal en cuestión se tiene

$$
|\mathcal{L}(v)|=\left|\int_{\Omega} v(\boldsymbol{r}) \nabla \cdot \boldsymbol{J}^{p}(\boldsymbol{r}) d \Omega\right| \leq \int_{\Omega}\left|v(\boldsymbol{r}) \nabla \cdot \boldsymbol{J}^{p}(\boldsymbol{r})\right| d \Omega=\left\|v(\boldsymbol{r}) \nabla \cdot \boldsymbol{J}^{p}(\boldsymbol{r})\right\|_{L^{1}(\Omega)} .
$$

Utilizando la desigualdad de Hölder

$$
\left\|v(\boldsymbol{r}) \nabla \cdot J^{p}\right\|_{L^{1}(\Omega)} \leq\|v(\boldsymbol{r})\|_{L^{2}(\Omega)}\left\|\nabla \cdot \boldsymbol{J}^{p}(\boldsymbol{r})\right\|_{L^{2}(\Omega)} \leq\left\|\nabla \cdot \boldsymbol{J}^{p}(\boldsymbol{r})\right\|_{L^{2}(\Omega)}\|v(\boldsymbol{r})\|_{H^{1}(\Omega)}
$$

por lo que se demuestra que la forma lineal dada es continua con $\Lambda=\left\|\nabla \cdot \boldsymbol{J}^{p}(\boldsymbol{r})\right\|_{L^{2}(\Omega)}$.

\section{A.2. Demostración de la expresión (2.30)}

Considerando la expresión (2.24) que denota la proyección del campo magnético secundario en un punto $\boldsymbol{r}_{j}$ y en la dirección $\check{\boldsymbol{n}}_{l}$, y reemplazando $u(\boldsymbol{r})$ por su versión 
discretizada $u_{h}(\boldsymbol{r})$ definido en la Sección 2.3.3 se tiene

$$
\begin{aligned}
\boldsymbol{B}_{s}\left(\boldsymbol{r}_{j}\right) \cdot \check{\boldsymbol{n}}_{l} & =\frac{\mu_{0}}{4 \pi} \int_{\Omega} \boldsymbol{\sigma}\left(\boldsymbol{r}^{\prime}\right) \boldsymbol{\nabla} u_{h}\left(\boldsymbol{r}^{\prime}\right) \cdot \gamma_{l}\left(\boldsymbol{r}_{j}, \boldsymbol{r}^{\prime}\right) d \boldsymbol{r}^{\prime} \\
& =\frac{\mu_{0}}{4 \pi} \sum_{\Omega_{e} \subset \Omega}\left(\int_{\Omega_{e}} \boldsymbol{\sigma}\left(\boldsymbol{r}^{\prime}\right) \boldsymbol{\nabla} u_{h}\left(\boldsymbol{r}^{\prime}\right) \cdot \gamma_{l}\left(\boldsymbol{r}_{j}, \boldsymbol{r}^{\prime}\right) d \boldsymbol{r}^{\prime}\right) \\
& =\frac{\mu_{0}}{4 \pi} \sum_{\Omega_{e} \subset \Omega}\left(\boldsymbol{\sigma}_{e} \int_{\Omega_{e}}\left(\sum_{i=1}^{N} \eta_{i} \boldsymbol{\nabla} \varphi_{i}\left(\boldsymbol{r}^{\prime}\right)\right) \cdot \boldsymbol{\gamma}_{l}\left(\boldsymbol{r}_{j}, \boldsymbol{r}^{\prime}\right) d \boldsymbol{r}^{\prime}\right) \\
& =\frac{\mu_{0}}{4 \pi} \sum_{\Omega_{e} \subset \Omega}\left(\boldsymbol{\sigma}_{e} \int_{\Omega_{e}}\left(\frac{1}{3 ! V_{e}} \boldsymbol{D}_{e} \boldsymbol{\eta}_{e}\right) \cdot \boldsymbol{\gamma}_{l}\left(\boldsymbol{r}_{j}, \boldsymbol{r}^{\prime}\right) d \boldsymbol{r}^{\prime}\right) \\
& =\frac{\mu_{0}}{4 \pi} \sum_{\Omega_{e} \subset \Omega}\left(\frac{1}{3 ! V_{e}} \boldsymbol{\sigma}_{e} \boldsymbol{D}_{e} \boldsymbol{\eta}_{e} \cdot \iota_{j}\right),
\end{aligned}
$$

en donde $\iota_{j}=\int_{\Omega_{e}} \boldsymbol{\gamma}_{l}\left(\boldsymbol{r}_{j}, \boldsymbol{r}^{\prime}\right) d \boldsymbol{r}^{\prime}$ es un vector columna de 3 elementos y $\boldsymbol{\eta}_{e}$ es un vector de 4 elementos correspondiente al potencial eléctrico en los cuatro nodos del elemento $e$. Sean $\boldsymbol{r}_{j}, j=1, \ldots, N_{s}$ los puntos en donde se pretende calcular el campo magnético, i.e. sensores de campo del equipo adquisidor de MEG. Luego, es posible definir las matrices $\boldsymbol{I}_{e}=\left[\iota_{1}, \cdots, \iota_{N_{s}}\right]^{T}$ de $N_{s} \times 3$ y $\boldsymbol{S}_{e}=\frac{\mu_{0}}{4 \pi 3 ! V_{e}} \boldsymbol{I}_{e} \boldsymbol{\sigma}_{e} \boldsymbol{D}_{e}$, resultando

$$
\boldsymbol{\vartheta}_{s}=\sum_{e=1}^{N_{e}} \boldsymbol{S}_{e} \boldsymbol{\eta}_{e}
$$

demostrando lo propuesto. 


\section{Apéndice B}

\section{Deducción de algunas fórmulas del Capítulo 3}

\section{B.1. Breve reseña sobre sLORETA}

Como se vio en el Capítulo 2, la solución del problema directo en EEG considerando $N_{e}$ electrodos y $N_{v}$ fuentes dipolares distribuidas en la corteza vendrá dada por

$$
\phi=K \boldsymbol{j}
$$

en donde $\phi$ es el vector de los potenciales eléctricos medidos, $\boldsymbol{j}$ es el vector de $3 N_{v}$ elementos correspondientes a la densidad de corriente primaria definido por $\boldsymbol{j}=\left(\boldsymbol{j}_{1}^{T}, \boldsymbol{j}_{2}^{T}, \ldots, \boldsymbol{j}_{N_{v}}^{T}\right)^{T}$, con $\boldsymbol{j}_{l}$ vector de 3 elementos correspondientes a la l-ésima fuente de corriente, y $\boldsymbol{K}$ es la matriz de lead-field, de dimensión $N_{e} \times 3 N_{v}$.

La solución buscada por el método de sLORETA resultará de resolver

$$
\widehat{\boldsymbol{j}}=\operatorname{mín}_{\boldsymbol{j}}\left\{\|\boldsymbol{\phi}-\boldsymbol{K} \boldsymbol{j}\|^{2}+\alpha\|\boldsymbol{j}\|^{2}\right\}
$$

en donde $\alpha>0$ es el parámetro de regularización de Tikhonov (Tikhonov y Arsenin, 1977) que permite restringir la norma de $\widehat{\boldsymbol{j}}$. Se muestra (Pascual-Marqui, 2002) que la solución de (B.1) es

$$
\widehat{j}=T \phi
$$


en donde $\boldsymbol{T}=\boldsymbol{K}^{T}\left[\boldsymbol{K} \boldsymbol{K}^{T}+\alpha \boldsymbol{H}\right]^{+}, \boldsymbol{H}=\boldsymbol{I}-\mathbf{1 1}^{T} / \mathbf{1}^{T} \mathbf{1}$ es el operador de referencia promedio, $\boldsymbol{I}$ es la matriz identidad de dimensión $N_{e}, \mathbf{1}$ es el vector de $N_{e}$ elementos lleno de unos, y el superíndice + denota a la pseudo-inversa de Moore-Penrose.

Si se asumen fuentes independientes entre sí $\left(\boldsymbol{S}_{\boldsymbol{j}}=\boldsymbol{I}_{3 N_{v}}\right)$ y respecto al ruido de las mediciones (de covarianza $\boldsymbol{S}_{\boldsymbol{\phi}}^{\text {noise }}=\alpha \boldsymbol{H}$ ), la matriz de covarianza del potencial eléctrico medido vendrá dada por (Michel et al., 2009)

$$
\boldsymbol{S}_{\boldsymbol{\phi}}=\boldsymbol{K} \boldsymbol{S}_{\boldsymbol{j}} \boldsymbol{K}^{T}+\boldsymbol{S}_{\boldsymbol{\phi}}^{\text {noise }}=\boldsymbol{K} \boldsymbol{K}^{T}+\alpha \boldsymbol{H}
$$

Luego, utilizando (B.2), la varianza de la densidad de corriente estimada será

$$
\boldsymbol{S}_{\widehat{\boldsymbol{j}}}=\boldsymbol{T} \boldsymbol{S}_{\boldsymbol{\phi}} \boldsymbol{T}=\boldsymbol{K}^{T}\left[\boldsymbol{K} \boldsymbol{K}^{T}+\alpha \boldsymbol{H}\right]^{+} \boldsymbol{K} .
$$

Finalmente, para inferir la ubicación de las fuentes corticales, el SCDP en el l-ésimo nodo de interés (sobre la corteza) se obtiene mediante el cálculo de

$$
\widehat{\boldsymbol{j}}_{l}^{T}\left\{\left[\boldsymbol{S}_{\widehat{\boldsymbol{j}}}\right]_{l}\right\}^{-1} \widehat{\boldsymbol{j}}_{l}
$$

en donde $\left[\boldsymbol{S}_{\widehat{j}}\right]_{l}$ es la matriz de de covarianza de $3 \times 3$ correspondiente a la densidad de corriente estimada en la l-ésima ubicación. Nótese que, bajo las suposiciones realizadas, $\left[\boldsymbol{S}_{\hat{\boldsymbol{j}}}\right]_{l}$ depende de $\boldsymbol{K}$ y $\boldsymbol{H}$, o lo que es igual, del modelo de cabeza y disposición de los electrodos. 


\section{Apéndice $\mathrm{C}$}

\section{Deducción de algunas fórmulas del Capítulo 5}

\section{C.1. Gradiente de potencial}

En este apéndice se muestran las expresiones de las derivadas $\partial \boldsymbol{\Phi}_{F} / \partial \boldsymbol{\theta}$ y $\partial \boldsymbol{f} / \partial \boldsymbol{\theta}$ mencionadas en la Sección 5.4. Si se considera una única fuente dipolar ubicada en $\boldsymbol{p}$ con momento dipolar $\boldsymbol{q}$, el vector de parámetros será el vector de seis elementos $\boldsymbol{\theta}=\left[\boldsymbol{p}^{T}, \boldsymbol{q}^{T}\right]^{T}$. Sea $\boldsymbol{x}_{i}^{a}, i=1 \ldots, N$ el conjunto de puntos del espacio en donde las derivadas son evaluadas, i.e. cada uno de los $N$ puntos del mallado correspondiente a la superficie interior. Luego, la $i$-ésima fila de $\partial \boldsymbol{\Phi}_{F} / \partial \boldsymbol{\theta}$ vendrá dada por $\partial \boldsymbol{\Phi}_{F}\left(\boldsymbol{x}_{i}^{a}\right) / \partial \boldsymbol{\theta}=\left[\boldsymbol{\nabla}_{\boldsymbol{p}}^{T} \boldsymbol{\Phi}_{F}\left(\boldsymbol{x}_{i}^{a}\right), \boldsymbol{\nabla}_{\boldsymbol{q}}^{T} \boldsymbol{\Phi}_{F}\left(\boldsymbol{x}_{i}^{a}\right)\right]$. Dado que $\boldsymbol{\Phi}_{F}\left(\boldsymbol{x}_{i}^{a}\right)=\left(\boldsymbol{q} \cdot \boldsymbol{r}_{i}\right) / 4 \pi r_{i}^{2}$ (Sarvas, 1987) es simple obtener

$$
\begin{aligned}
\boldsymbol{\nabla}_{\boldsymbol{p}} \boldsymbol{\Phi}_{F}\left(\boldsymbol{x}_{i}^{a}\right) & =\frac{1}{4 \pi r_{i}^{3}}\left(3\left(\boldsymbol{q} \cdot \boldsymbol{r}_{i}\right) \boldsymbol{r}_{i}-\boldsymbol{q}\right) \\
\boldsymbol{\nabla}_{\boldsymbol{q}} \boldsymbol{\Phi}_{F}\left(\boldsymbol{x}_{i}^{a}\right) & =\frac{1}{4 \pi r_{i}^{2}} \boldsymbol{r}_{i}
\end{aligned}
$$

en donde $r_{i}=\left|\boldsymbol{x}_{i}^{a}-\boldsymbol{p}\right|$ y $\boldsymbol{r}_{i}=\left(\boldsymbol{x}_{i}^{a}-\boldsymbol{p}\right) /\left|\boldsymbol{x}_{i}^{a}-\boldsymbol{p}\right|$.

Por otro lado, considérese la matriz $\partial \boldsymbol{f} / \partial \boldsymbol{\theta}$ de dimensión $2 N \times 6$, cuya i-ésima fila viene dada por $\left[\boldsymbol{\nabla}_{\boldsymbol{p}}^{T} f_{i}, \boldsymbol{\nabla}_{\boldsymbol{q}}^{T} f_{i}\right]$, con $\boldsymbol{f}=\left\{f_{i}\right\}_{i}$ definido en (C.4). Como se muestra en el Apéndice C.2, existe una relación lineal entre $f_{i}$ y $\boldsymbol{J}_{F_{i}}$. En von Ellenrieder et al. 
(2005a) se muestra que

$$
\boldsymbol{J}_{F_{i}}=\frac{1}{4 \pi r_{i}^{3}}\left[\boldsymbol{q}-3\left(\boldsymbol{q} \cdot \boldsymbol{r}_{i}\right) \boldsymbol{r}_{i}\right]
$$

Luego,

$$
\begin{aligned}
& \nabla_{\boldsymbol{p}} f_{i}=\frac{3}{4 \pi r_{i}^{4}}\left[\boldsymbol{r}_{i}\left(\boldsymbol{t}_{i}^{\gamma} \cdot \boldsymbol{q}\right)-\boldsymbol{r}_{i}\left(\boldsymbol{q} \cdot \boldsymbol{r}_{i}\right)\left(\boldsymbol{t}_{i}^{\gamma} \cdot \boldsymbol{r}_{i}\right)+\boldsymbol{q}\left(\boldsymbol{t}_{i}^{\gamma} \cdot \boldsymbol{r}_{i}\right)\right. \\
& \left.+\boldsymbol{q}\left(\boldsymbol{t}_{i}^{\gamma} \cdot \boldsymbol{r}_{i}\right)+\left(\boldsymbol{q} \cdot \boldsymbol{r}_{i}\right) \boldsymbol{t}_{i}^{\gamma}\right], \\
& \boldsymbol{\nabla}_{\boldsymbol{q}} f_{i}=\frac{1}{4 \pi r_{i}^{3}}\left[\boldsymbol{t}_{i}^{\gamma}-3\left(\boldsymbol{t}_{i}^{\gamma} \cdot \boldsymbol{r}_{i}\right) \boldsymbol{r}_{i}\right] \text {, }
\end{aligned}
$$

en donde $\gamma=a$ si $i=1, \cdots, N$ y $\gamma=b$ si $i=N+1, \cdots, 2 N$, y $\boldsymbol{r}_{i}=\boldsymbol{r}_{i-N}$ si $i=N+1, \cdots, 2 N$.

\section{C.2. Problema directo del gradiente}

En este apéndice se muestra la formulación del problema directo del gradiente definida en (5.11). En principio es necesario encontrar una expresión para $\boldsymbol{\nabla} \Phi(\mathbb{S})$. De von Ellenrieder et al. (2005a) se sabe que el gradiente del potencial eléctrico en un punto $\boldsymbol{s}_{i}$ del mallado $\mathbb{S}$ de $\Omega$ está dado por

$$
\boldsymbol{\nabla} \varphi\left(\boldsymbol{s}_{i}\right)=\frac{1}{\sigma_{M}}\left(\boldsymbol{n}\left(\boldsymbol{s}_{i}\right) \times \boldsymbol{K}\left(\boldsymbol{s}_{i}\right)\right),
$$

en donde $\sigma_{M}$ es la conductividad eléctrica de la capa exterior (cuero cabelludo), $\boldsymbol{n}\left(\boldsymbol{s}_{i}\right)$ es el vector unitario normal a $\Omega$ en $\boldsymbol{s}_{i}$, y $\boldsymbol{K}\left(\boldsymbol{s}_{i}\right)=\left[k_{i}^{a}, k_{i}^{b}\right]$ es la densidad de corriente equivalente en el sistema de coordenadas locales $\left\{\boldsymbol{t}_{i}^{a}, \boldsymbol{t}_{i}^{b}\right\}$. Se muestra fácilmente que, siendo $\left(w_{i}, z_{i}, 0\right)$ las coordenadas de $\boldsymbol{s}_{i}$ en el sistema de coordenadas locales, y basados en (C.1), el gradiente del potencial en dicho punto viene dado por

$$
\boldsymbol{\nabla} \varphi\left(\boldsymbol{s}_{i}\right)=\left[\begin{array}{c}
\frac{\partial \varphi(\boldsymbol{\chi})}{\partial w_{i}} \mid \\
\left.\frac{\partial \varphi(\boldsymbol{\chi})}{\partial z_{i}}\right|_{\boldsymbol{s}_{i}}
\end{array} \boldsymbol{s}_{i}\right]=\frac{1}{\sigma_{M}}\left[\begin{array}{c}
-k_{i}^{b} \\
k_{i}^{a}
\end{array}\right],
$$

en donde la componente normal no se presenta por ser siempre cero. Reemplazando (C.2) en $\boldsymbol{\nabla} \boldsymbol{\Phi}(\mathbb{S})$ lleva a

$$
\boldsymbol{\nabla} \boldsymbol{\Phi}(\mathbb{S})=\frac{1}{\sigma_{M}}\left[\begin{array}{ccc}
-k_{1}^{b} & \ldots & -k_{N}^{b} \\
k_{1}^{a} & \ldots & k_{N}^{a}
\end{array}\right]^{T} .
$$


Basado en las definiciones originales descriptas en von Ellenrieder et al. (2005a) se definen la matriz $\boldsymbol{G}_{\Delta}$ y el vector $\boldsymbol{f}$ como

$$
\begin{aligned}
& \boldsymbol{G}_{\Delta}=\left[\begin{array}{cc}
\boldsymbol{G}_{D} & -\boldsymbol{G}_{C} \\
-\boldsymbol{G}_{B} & \boldsymbol{G}_{A}
\end{array}\right], \\
& \boldsymbol{f}=\left[\boldsymbol{t}_{1}^{a} \cdot \boldsymbol{J}_{F_{1}}, \ldots, \boldsymbol{t}_{N}^{a} \cdot \boldsymbol{J}_{F_{N}}, \boldsymbol{t}_{1}^{b} \cdot \boldsymbol{J}_{F_{1}}, \ldots, \boldsymbol{t}_{N}^{b} \cdot \boldsymbol{J}_{F_{N}}\right]^{T},
\end{aligned}
$$

en donde $\boldsymbol{J}_{F_{i}}$ es el efecto de la fuente dipolar en coordenadas locales sobre el $i$-ésimo elemento y, a su vez, es el único término dependiente de los parámetros de la fuente. La matriz $\boldsymbol{G}_{\Delta}$ sólo depende de la geometría del modelo, y se encuentra definida por

$$
\begin{aligned}
& G_{A i j}=\boldsymbol{t}_{i}^{b} \cdot\left(\vec{\Omega}_{i j} \cdot \boldsymbol{t}_{j}^{a} \boldsymbol{n}_{j}-\Omega_{i j} \boldsymbol{t}_{j}^{b}\right), \\
& G_{B i j}=\boldsymbol{t}_{i}^{b} \cdot\left(\vec{\Omega}_{i j} \cdot \boldsymbol{t}_{j}^{b} \boldsymbol{n}_{j}+\Omega_{i j} \boldsymbol{t}_{j}^{a}\right), \\
& G_{C i j}=-\boldsymbol{t}_{i}^{a} \cdot\left(\vec{\Omega}_{i j} \cdot \boldsymbol{t}_{j}^{a} \boldsymbol{n}_{j}-\Omega_{i j} \boldsymbol{t}_{j}^{b}\right), \\
& G_{D i j}=-\boldsymbol{t}_{i}^{a} \cdot\left(\vec{\Omega}_{i j} \cdot \boldsymbol{t}_{j}^{b} \boldsymbol{n}_{j}+\Omega_{i j} \boldsymbol{t}_{j}^{a}\right),
\end{aligned}
$$

en donde

$$
\begin{aligned}
& \Omega_{i j}=\frac{1}{4 \pi} \int_{\triangle_{j}} \nabla\left(\frac{1}{r_{i}}\right) \cdot \boldsymbol{n}_{j} d s_{j}, \\
& \vec{\Omega}_{i j}=\frac{1}{4 \pi} \int_{\triangle_{j}} \nabla\left(\frac{1}{r_{i}}\right) \times \boldsymbol{n}_{j} d s_{j},
\end{aligned}
$$

siendo $\triangle_{j}$ el j-ésimo triángulo del mallado. Luego, se llega a un sistema lineal cuya solución se encuentra dada por

$$
\left[\begin{array}{c}
-\boldsymbol{k}^{b} \\
\boldsymbol{k}^{a}
\end{array}\right]=-\left(\boldsymbol{\Lambda}_{K}-\boldsymbol{G}_{\Delta}\right)^{-1} \boldsymbol{f}
$$

con $\boldsymbol{k}^{a}=\left[k_{1}^{a}, \ldots, k_{N}^{a}\right]^{T}$ y $\boldsymbol{k}^{b}=\left[k_{1}^{b}, \ldots, k_{N}^{b}\right]^{T}$. Reemplazando (C.5) en (C.3), se encuentra una expresión para $\operatorname{vec}(\boldsymbol{\nabla} \boldsymbol{\Phi}(\mathbb{S}))$,

$$
\operatorname{vec}(\boldsymbol{\nabla} \boldsymbol{\Phi}(\mathbb{S}))=\boldsymbol{M} \boldsymbol{f}
$$

en donde $\boldsymbol{M}=-\frac{1}{\sigma_{M}}\left(\boldsymbol{\Lambda}_{K}-\boldsymbol{G}_{\Delta}\right)^{-1}$. 


\section{C.3. Deducción de (5.12)}

En este apéndice se muestra (5.12). Tal como se mencionó en la Sección 5.4, la matriz de interpolación $\boldsymbol{H}_{F}(\boldsymbol{x})$ permite escribir a la matriz de gradiente de potencial como

$$
\nabla \Phi(\boldsymbol{x})=\boldsymbol{H}_{F}(\boldsymbol{x}) \nabla \Phi(\mathbb{S}) .
$$

Utilizando la definición de $\boldsymbol{D}$ dada en la Sección 5.2 y reemplazándola en (C.7) se llega a

$$
\boldsymbol{D}=\operatorname{diag}\left\{\operatorname{vec}\left(\boldsymbol{\nabla} \boldsymbol{\Phi}\left(\boldsymbol{x}_{0}\right)\right)\right\}=\operatorname{diag}\left\{\operatorname{vec}\left(\boldsymbol{H}_{F}\left(\boldsymbol{x}_{0}\right) \boldsymbol{\nabla} \boldsymbol{\Phi}(\mathbb{S})\right)\right\} .
$$

Considerando el hecho de que $\operatorname{vec}(\boldsymbol{A B})=\left(\boldsymbol{I}_{p} \otimes \boldsymbol{A}\right) \operatorname{vec}(\boldsymbol{B})$ para $\boldsymbol{A}_{m \times n}$ y $\boldsymbol{B}_{n \times p}, \mathrm{y}$ reemplazando (C.6) en (C.8) se obtiene

$$
\begin{aligned}
\boldsymbol{D} & =\operatorname{diag}\left\{\left(\boldsymbol{I}_{2} \otimes \boldsymbol{H}_{F}\left(\boldsymbol{x}_{0}\right)\right) \operatorname{vec}(\boldsymbol{\nabla} \boldsymbol{\Phi}(\mathbb{S}))\right\} \\
& =\operatorname{diag}\left\{\left(\boldsymbol{I}_{2} \otimes \boldsymbol{H}_{F}\left(\boldsymbol{x}_{0}\right)\right) \boldsymbol{M} \boldsymbol{f}\right\}
\end{aligned}
$$

Puede observarse que el único factor de $\boldsymbol{D}$ dependiente del vector de parámetros de la fuente $\boldsymbol{\theta}$ es $\boldsymbol{f}$. Luego, su derivada es

$$
\frac{\partial \boldsymbol{D}}{\partial \theta_{j}}=\operatorname{diag}\left\{\left(\boldsymbol{I}_{2} \otimes \boldsymbol{H}_{F}\left(\boldsymbol{x}_{0}\right)\right) \boldsymbol{M} \frac{\partial \boldsymbol{f}}{\partial \theta_{j}}\right\} .
$$

Luego, se busca una expresión para $\boldsymbol{J}^{1}$. Empleando la regla de la cadena y sabiendo que el único término de $\mathbb{C}_{\boldsymbol{\Phi}}$ que depende de la fuente es $\boldsymbol{D}$, se obtiene

$$
\frac{\partial \mathbb{C}_{\boldsymbol{\Phi}}}{\partial \theta_{j}}=\boldsymbol{E} \frac{\partial\left(\boldsymbol{D} \mathbb{C}_{\boldsymbol{x}} \boldsymbol{D}\right)}{\partial \theta_{j}} \boldsymbol{E}^{T}=\boldsymbol{E}\left(\frac{\partial \boldsymbol{D}}{\partial \theta_{j}} \mathbb{C}_{\boldsymbol{x}} \boldsymbol{D}+\boldsymbol{D} \mathbb{C}_{\boldsymbol{x}} \frac{\partial \boldsymbol{D}}{\partial \theta_{j}}\right) \boldsymbol{E}^{T},
$$

en donde se utilizó el hecho de que $\boldsymbol{D}$ es diagonal. Si se asume independencia entre las posiciones de los sensores, de la Sección 5.2 se tiene $\mathbb{C}_{\boldsymbol{x}}=\sigma^{2} \boldsymbol{I}_{2 N_{S}}$. Reemplazando las ecuaciones (C.9) y (C.10) en (C.11) se llega a

$$
\begin{aligned}
\frac{\partial \mathbb{C}_{\boldsymbol{\Phi}}}{\partial \theta_{j}} & =2 \sigma^{2} \boldsymbol{E} \operatorname{diag}\left\{\operatorname{vec}\left(\boldsymbol{\nabla} \boldsymbol{\Phi}\left(\boldsymbol{x}_{0}\right)\right)\right\} \operatorname{diag}\left\{\operatorname{vec}\left(\frac{\partial \boldsymbol{\nabla} \boldsymbol{\Phi}\left(\boldsymbol{x}_{0}\right)}{\partial \theta_{j}}\right)\right\} \boldsymbol{E}^{T} \\
& =2 \sigma^{2} \boldsymbol{E} \operatorname{diag}\left\{\operatorname{vec}\left(\boldsymbol{\nabla} \boldsymbol{\Phi}\left(\boldsymbol{x}_{0}\right)\right) \circ \operatorname{vec}\left(\frac{\partial \boldsymbol{\nabla}\left(\boldsymbol{x}_{0}\right)}{\partial \theta_{j}}\right)\right\} \boldsymbol{E}^{T} \\
& =2 \sigma^{2} \boldsymbol{E} \operatorname{diag}\left\{\left[\left(\boldsymbol{I}_{2} \otimes \boldsymbol{H}_{F}^{0}\right) \boldsymbol{M} \boldsymbol{f}\right] \circ\left[\left(\boldsymbol{I}_{2} \otimes \boldsymbol{H}_{F}^{0}\right) \boldsymbol{M} \frac{\partial \boldsymbol{f}}{\partial \theta_{j}}\right]\right\} \boldsymbol{E}^{T},
\end{aligned}
$$

en donde $\boldsymbol{H}_{F}^{0}=\boldsymbol{H}_{F}\left(\boldsymbol{x}_{0}\right)$. 


\section{Apéndice D}

\section{Deducción de algunas fórmulas del Capítulo 6}

\section{D.1. Demostración del teorema 6.3.1}

En principio se debe mostrar que $\mathbb{E}\left\{\left\|\widehat{\boldsymbol{\Omega}}_{S}-\boldsymbol{\Omega}\right\|_{F}^{2}\right\} \leq \mathbb{E}\left\{\left\|\boldsymbol{S}_{\Omega}-\boldsymbol{\Omega}\right\|_{F}^{2}\right\}$. Aplicando sucesivamente la propiedad de la norma Frobenius $\|\boldsymbol{A}+\boldsymbol{B}\|_{F}^{2}=\|\boldsymbol{A}\|_{F}^{2}+\|\boldsymbol{B}\|_{F}^{2}+$ $2 \operatorname{tr}\left\{\boldsymbol{A B}^{T}\right\}$ para $\boldsymbol{A}$ y $\boldsymbol{B}$ matrices cuadradas (Horn y Johnson, 1985), y utilizando definiciones previas, se llega a

$$
\mathbb{E}\left\{\left\|\widehat{\boldsymbol{\Omega}}_{S}-\boldsymbol{\Omega}\right\|_{F}^{2}\right\}=\mathbb{E}\left\{\left\|\boldsymbol{S}_{\Omega}-\boldsymbol{\Omega}\right\|_{F}^{2}\right\}-\left(\alpha_{\Omega}^{o}\right)^{2} \mathbb{E}\left\{\left\|\boldsymbol{T}_{\Omega}-\boldsymbol{S}_{\Omega}\right\|_{F}^{2}\right\}
$$

la cual es menor o igual a $\mathbb{E}\left\{\left\|\boldsymbol{S}_{\Omega}-\boldsymbol{\Omega}\right\|_{F}^{2}\right\}$. Debe notarse que la igualdad se da en caso de que $\alpha_{\Omega}^{o}=0$ se cumpla, y que valores de $\alpha_{\Omega}^{o}$ mayores implicarán mejoras del estimador de constricción respecto de los estimadores muestrales insesgados.

Luego, debe mostrarse que $\mathbb{E}\left\{\left\|\widehat{\boldsymbol{\Omega}}_{S}-\boldsymbol{\Omega}\right\|_{F}^{2}\right\} \rightarrow 0$ si $K \rightarrow \infty$. Dada la primer parte del teorema, es suficiente probar que $\boldsymbol{S}_{\Omega}$ es consistente bajo la norma Frobenius. Se define $X_{p n}^{k}=Y_{p n}^{k}-M_{p n}$ por comodidad en la notación. Asimismo, sean $\omega_{i j}$ y $s_{i j}$, $i, j=1, \ldots, P$, los elementos de las matrices $\Omega$ y $\boldsymbol{S}_{\Omega}$, respectivamente. Resulta 
$s_{i j}=1 / N(K-1) \sum_{k=1}^{K} \sum_{n=1}^{N} X_{i n}^{k} X_{j n}^{k}$. Luego,

$$
\begin{array}{r}
\mathbb{E}\left\{\left\|\boldsymbol{S}_{\Omega}-\boldsymbol{\Omega}\right\|_{F}^{2}\right\}=\sum_{i, j=1}^{P} \mathbb{E}\left\{\left(s_{i j}-\omega_{i j}\right)^{2}\right\}= \\
\frac{1}{K^{2}} \sum_{i, j=1}^{P} \mathbb{E}\left\{\left[\sum_{k=1}^{K}\left(\frac{K}{N(K-1)} \sum_{n=1}^{N} X_{i n}^{k} X_{j n}^{k}-\omega_{i j}\right)\right]^{2}\right\}= \\
\frac{K}{N^{2}(K-1)^{2}} \sum_{i, j=1}^{P} \mathbb{E}\left\{\left(\sum_{n=1}^{N} X_{i n}^{k} X_{j n}^{k}\right)^{2}\right\}-\frac{1}{K}\|\Omega\|_{F}^{2},
\end{array}
$$

en donde se utilizó el hecho de que las diferentes realizaciones se supusieron independientes. Haciendo uso de la desigualdad entre las medias aritmética y geométrica, y la desigualdad de Cauchy, es fácil probar que

$$
\begin{array}{r}
\sum_{i, j=1}^{P} \mathbb{E}\left\{\left(\sum_{n=1}^{N} X_{i n}^{k} X_{j n}^{k}\right)^{2}\right\} \leq \sum_{i, j=1}^{P} \mathbb{E}\left\{\left(\sum_{n=1}^{N}\left(X_{i n}^{k}\right)^{2}\right)\left(\sum_{n=1}^{N}\left(X_{j n}^{k}\right)^{2}\right)\right\} \\
\leq \frac{P}{2}\left[\mathbb{E}\left\{\sum_{i=1}^{P} \sum_{n=1}^{N}\left(X_{i n}^{k}\right)^{2}\right\}+\mathbb{E}\left\{\sum_{j=1}^{P} \sum_{n=1}^{N}\left(X_{j n}^{k}\right)^{2}\right\}\right] \leq P K_{1}^{2}
\end{array}
$$

en donde $K_{1}$ es una constante independiente de $K$. Luego,

$$
\mathbb{E}\left\{\left\|\boldsymbol{S}_{\Omega}-\Omega\right\|_{F}^{2}\right\} \leq \frac{P K_{1}^{2}}{N^{2}} \frac{K}{(K-1)^{2}}-\frac{1}{K}\|\Omega\|_{F}^{2},
$$

y el término derecho de la desigualdad tiende a cero a medida que $K$ tiene a infinito.

Igual camino debe tomarse para la demostración considerando el caso temporal.

\section{D.2. Demostración del teorema 6.3.2}

Por la desigualdad de Weyl (Horn y Johnson, 1985) se sabe que si $\boldsymbol{A}$ y $\boldsymbol{B}$ son dos matrices hermíticas de $R \times R$, luego $\lambda_{r}(\boldsymbol{A})+\lambda_{m}(\boldsymbol{B}) \leq \lambda_{r}(\boldsymbol{A}+\boldsymbol{B}) \leq \lambda_{r}(\boldsymbol{A})+\lambda_{M}(\boldsymbol{B})$ se cumple para $r=1, \ldots, R$. Reemplazando $\boldsymbol{A}=\alpha_{\Sigma} \boldsymbol{T}_{\Sigma}$ y $\boldsymbol{B}=\left(1-\alpha_{\Sigma}\right) \boldsymbol{S}_{\Sigma}$, y notando que $\boldsymbol{A}+\boldsymbol{B}=\widehat{\boldsymbol{\Sigma}}_{S}$, se muestra la desigualdad (6.10). El mismo procedimiento debe seguirse para probar (6.11). 


\section{D.3. Obtención de la cota de Cramér-Rao}

En este apéndice se derivan las expresiones (6.17) y (6.18). Tal como se mencionó en la Sección 6.1, se considera una distribución matricial Gaussiana para el ruido de fondo. Luego, la matriz de información de Fisher se obtiene reemplazando $\Upsilon$ por $\boldsymbol{\Sigma} \otimes \Omega$ en (6.16),

$$
J_{i j}=\frac{1}{2} \operatorname{tr}\left\{\left(\boldsymbol{\Sigma}^{-1} \otimes \boldsymbol{\Omega}^{-1}\right) \frac{\partial(\boldsymbol{\Sigma} \otimes \boldsymbol{\Omega})}{\partial \gamma_{i}}\left(\boldsymbol{\Sigma}^{-1} \otimes \boldsymbol{\Omega}^{-1}\right) \frac{\partial(\boldsymbol{\Sigma} \otimes \boldsymbol{\Omega})}{\partial \gamma_{j}}\right\} .
$$

Luego, utilizando algunas propiedades básicas de la traza y el producto de Kronecker (Horn y Johnson, 1985), la matriz de información de Fisher toma la forma

$$
\boldsymbol{J}=\left(\begin{array}{ll}
\boldsymbol{J}_{A} & \boldsymbol{J}_{B} \\
\boldsymbol{J}_{B}^{T} & \boldsymbol{J}_{C}
\end{array}\right)
$$

en donde

$$
\begin{array}{r}
J_{A_{i j}}=\frac{K P}{2} \operatorname{tr}\left\{\boldsymbol{\Sigma}^{-1} \frac{\partial \boldsymbol{\Sigma}}{\partial \gamma_{\sigma_{i}}} \boldsymbol{\Sigma}^{-1} \frac{\partial \boldsymbol{\Sigma}}{\partial \gamma_{\sigma_{j}}}\right\}, \\
J_{B_{i j}}=\frac{K}{2} \operatorname{tr}\left\{\boldsymbol{\Sigma}^{-1} \frac{\partial \boldsymbol{\Sigma}}{\partial \gamma_{\sigma_{i}}}\right\} \operatorname{tr}\left\{\boldsymbol{\Omega}^{-1} \frac{\partial \boldsymbol{\Omega}}{\partial \gamma_{\omega_{j}}}\right\}, \\
J_{C_{i j}}=\frac{K N}{2} \operatorname{tr}\left\{\boldsymbol{\Omega}^{-1} \frac{\partial \boldsymbol{\Omega}}{\partial \gamma_{\omega_{i}}} \boldsymbol{\Omega}^{-1} \frac{\partial \boldsymbol{\Omega}}{\partial \gamma_{\omega_{j}}}\right\} .
\end{array}
$$

Usando (6.15), y aplicando la fórmula de inversión de matrices definidas por bloques (Horn y Johnson, 1985), se puede descomponer $\operatorname{CRB}(\gamma)$ en

$$
\operatorname{CRB}(\gamma)=\left(\begin{array}{ll}
\mathbf{C R B}_{\sigma \sigma} & \mathbf{C R B}_{\sigma \omega} \\
\mathbf{C R B}_{\omega \sigma} & \mathbf{C R B}_{\omega \omega}
\end{array}\right)
$$

en donde $\mathbf{C R B}_{\sigma \sigma}=\left(\boldsymbol{J}_{A}-\boldsymbol{J}_{B} \boldsymbol{J}_{C}^{-1} \boldsymbol{J}_{B}^{T}\right)^{-1}$ y $\mathbf{C R B}_{\omega \omega}=\left(\boldsymbol{J}_{C}-\boldsymbol{J}_{B}^{T} \boldsymbol{J}_{A}^{-1} \boldsymbol{J}_{B}\right)^{-1}$. Debe notarse que en el caso de que sólo $\boldsymbol{J}_{C}$ sea invertible, el ABCD-lema (Horn y Johnson, 1985) permite escribir $\mathbf{C R B}_{\omega \omega}=\boldsymbol{J}_{C}^{-1}+\boldsymbol{J}_{C}^{-1} \boldsymbol{J}_{B}^{T} \mathbf{C R B}_{\sigma \sigma} \boldsymbol{J}_{B} \boldsymbol{J}_{C}^{-1}$. Luego, resulta fácil la descomposición de (6.15) en dos desigualdades, i.e. $\operatorname{Cov}\left(\boldsymbol{\gamma}_{\sigma}\right) \geq \mathbf{C R B}_{\sigma \sigma} \mathrm{y}$ $\operatorname{Cov}\left(\boldsymbol{\gamma}_{\omega}\right) \geq \mathbf{C R B}_{\omega \omega}$. Dado que los elementos de la diagonal de una matriz definida positiva son positivos, se puede mostrar que

$$
\begin{array}{r}
\mathbb{E}\left\{\left\|\widehat{\boldsymbol{\Sigma}}_{S}-\boldsymbol{\Sigma}\right\|_{F}^{2}\right\}=\sum_{i, j=1}^{N} \operatorname{Var}\left\{\widehat{\boldsymbol{\Sigma}}_{i j}\right\}=\boldsymbol{\rho}_{\sigma}^{T} \operatorname{diag}\left\{\operatorname{Cov}\left(\boldsymbol{\gamma}_{\sigma}\right)\right\} \geq \\
\geq \boldsymbol{\rho}_{\sigma}^{T} \operatorname{diag}\left\{\mathbf{C R B}_{\sigma \sigma}\right\}=\boldsymbol{\rho}_{\sigma}^{T} \operatorname{diag}\left\{\left(\boldsymbol{J}_{A}-\boldsymbol{J}_{B} \boldsymbol{J}_{C}^{-1} \boldsymbol{J}_{B}^{T}\right)^{-1}\right\},
\end{array}
$$


en donde $\boldsymbol{\rho}_{\sigma}$ es el vector de $N(N+1) / 2 \times 1$ cuyos elementos valen 2 a menos que $\gamma_{\sigma_{i}}=\Sigma_{i i}$, en cuyo caso valen 1 . La ecuación (6.18) puede probarse del mismo modo, utilizando al vector $\boldsymbol{\rho}_{\omega}$ de $P(P+1) / 2 \times 1$ cuyos elementos valen 2 a menos que $\gamma_{\omega_{i}}=\Omega_{i i}$, en cuyo caso valen 1 .

Finalmente, es preciso notar que el cálculo de (D.2), (D.3) y (D.4) puede realizarse de un modo sencillo. Sean $a_{i j}$ los elementos de la matriz invertible $\boldsymbol{A}$ y $a^{i j}$ los elementos de $\boldsymbol{A}^{-1}$. Luego,

$$
\operatorname{tr}\left\{\boldsymbol{A}^{-1} \frac{\partial \boldsymbol{A}}{\partial a_{p q}}\right\}=\left\{\begin{array}{rr}
2 a^{p q} & \text { si } p \neq q \\
a^{p p} & \text { si } p=q
\end{array}\right.
$$

y

$$
\operatorname{tr}\left\{\boldsymbol{A}^{-1} \frac{\partial \boldsymbol{A}}{\partial a_{p q}} \boldsymbol{A}^{-1} \frac{\partial \boldsymbol{A}}{\partial a_{r s}}\right\}=\left\{\begin{aligned}
2\left(a^{q r} a^{p s}+a^{p r} a^{q s}\right) & \text { si } p \neq q, r \neq s \\
2 a^{p r} a^{q r} & \text { si } p \neq q, r=s \\
\left(a^{p r}\right)^{2} & \text { si } p=q, r=s
\end{aligned}\right.
$$

por lo que el cálculo de $\boldsymbol{J}_{A}, \boldsymbol{J}_{B}$ y $\boldsymbol{J}_{C}$ resulta evidente. 


\section{Apéndice E}

\section{Deducción de algunas fórmulas del Capítulo 8}

\section{E.1. Cálculo de $J$}

En este apéndice se demuestra la expresión (8.4). Por definición, la matriz de información de Fisher está dada por

$$
J_{i j}=\mathbb{E}\left\{\frac{\partial^{2} \log (\mathcal{L})}{\partial \theta_{i} \partial \theta_{j}}\right\},
$$

en donde $\mathcal{L}$ es la función de verosimilitud de las mediciones y $\boldsymbol{\theta}=\left[\theta_{1}, \ldots, \theta_{p}\right]$ es el vector que contiene los $p$ parámetros a estimar. En el presente caso dichos parámetros son los elementos de $\boldsymbol{D}$ por lo que, dada la simetría del tensor, $p=6$ y los elementos de $\boldsymbol{\theta}$ son elegidos como

$$
\boldsymbol{D}=\left(\begin{array}{ccc}
\theta_{1} & \theta_{4} & \theta_{5} \\
\theta_{4} & \theta_{2} & \theta_{6} \\
\theta_{5} & \theta_{6} & \theta_{3}
\end{array}\right)
$$

Con la finalidad de calcular $\log (\mathcal{L})$ se asumen $N$ mediciones independientes e idénticamente distribuidas, cada una correspondiente a un gradiente de campo aplicado. Luego, basados en (8.2), es posible mostrar que la función de verosimilitud 
logarítmica es

$$
\log (\mathcal{L})=\sum_{n=1}^{N}\left(\log \left(\frac{A_{T n}^{1-L} S_{T n}^{L}}{\sigma^{2}}\right)+\log \left(I_{L-1}\left(\frac{A_{T n k} S_{T n}}{\sigma^{2}}\right)\right)-\frac{S_{T n}^{2}+A_{T n}^{2}}{2 \sigma^{2}}\right)
$$

Como se sabe, el teorema de Cramér-Rao establece como condición necesaria la llamada condición de regularidad, la cual establece que $\mathbb{E}\left\{\frac{\partial \log (\mathcal{L})}{\partial \theta_{i}}\right\}=0$. Tras algunos cálculos puede mostrarse que la condición de regularidad es

$$
\mathbb{E}\left\{S_{T n} \frac{I_{L}(\beta)}{I_{L-1}(\beta)}\right\}=A_{T n}
$$

en donde se consideró $\beta=A_{T n} S_{T n} / \sigma^{2}$ por conveniencia en la notación. Basados en (8.2), se ve claramente que esta condición se cumple siempre.

El siguiente paso consiste en tomar las derivadas de (E.2) y calcular su esperanza. Nuevamente, tras algunos cálculos se puede llegar a

$$
J_{i j}=\sum_{n=1}^{N} \frac{1}{\sigma^{4}} \frac{\partial A_{T n}}{\partial \theta_{i}} \frac{\partial A_{T n}}{\partial \theta_{j}}\left(\mathbb{E}\left\{S_{T n}^{2} \frac{I_{L}^{2}(\beta)}{I_{L-1}^{2}(\beta)}\right\}-A_{T n}^{2}\right)
$$

Sea $\eta_{n}=A_{n} / \sigma$ la SNR de la $n$-ésima medición y $\boldsymbol{G}_{\eta}$ la matriz de $6 \times N$ definida por $\boldsymbol{G}_{\eta}=\left[\eta_{1} \boldsymbol{g}_{1}, \cdots, \eta_{N} \boldsymbol{g}_{N}\right]$, en donde cada $\boldsymbol{g}_{n}$ es un vector columna de 6 elementos dado por

$$
\boldsymbol{g}_{n}=b\left[\gamma_{x n}^{2}, \gamma_{y n}^{2}, \gamma_{z n}^{2}, 2 \gamma_{x n} \gamma_{y n}, 2 \gamma_{x n} \gamma_{z n}, 2 \gamma_{y n} \gamma_{z n}\right]^{T}
$$

y $\left[\gamma_{x n}, \gamma_{y n}, \gamma_{z n}\right]^{T}$ es la dirección del $n$-ésimo gradiente de campo aplicado. Luego, utilizando (E.3) y definiciones de la sección 8.2.1, la matriz de información de Fisher resulta ser

$$
\boldsymbol{J}=C_{L}^{2}(\boldsymbol{p}) \boldsymbol{G}_{\eta} \Upsilon \boldsymbol{\boldsymbol { G } _ { \eta } ^ { T }}
$$

en donde $\boldsymbol{\Upsilon}$ es una matriz diagonal con $\{\boldsymbol{\Upsilon}\}_{i i}=\left(C_{L}(\boldsymbol{p}) \eta_{i}\right)^{1-L} Z\left(\eta_{i}, L, \boldsymbol{p}\right)-C_{L}^{2}(\boldsymbol{p}) \eta_{i}^{2}$ y

$$
Z\left(\eta_{i}, L, \boldsymbol{p}\right)=\int_{0}^{\infty} x^{2+L} \exp \left(-\frac{x^{2}+C_{L}^{2}(\boldsymbol{p}) \eta_{i}^{2}}{2}\right) \frac{I_{L}^{2}\left(x C_{L}(\boldsymbol{p}) \eta_{i}\right)}{I_{L-1}\left(x C_{L}(\boldsymbol{p}) \eta_{i}\right)} d x
$$




\section{E.2. Cálculo de las derivadas de los autovalores respecto a $\theta$}

En este apéndice se muestran expresiones para los autovalores de $\boldsymbol{D}$ y sus derivadas con respecto a $\boldsymbol{\theta}$. Esto se hace mediante la resolución trigonométrica del polinomio cúbico propuesta por Cardano (Smith, 1961). Sea $m=\frac{1}{3} \operatorname{tr}\{\boldsymbol{D}\}$, $q=\frac{1}{2} \operatorname{det}\left(\boldsymbol{D}-m \boldsymbol{I}_{3}\right), p=\frac{1}{6}\left\|\boldsymbol{D}-m \boldsymbol{I}_{3}\right\|_{F}^{2}$ y $\varphi=\frac{1}{3} \arccos \left(q / p^{3 / 2}\right)$. Luego, como $\boldsymbol{D}$ es simétrico, su autovalores son reales y vienen dados por (Smith, 1961)

$$
\begin{aligned}
& \lambda_{1}=m+2 \sqrt{p} \cos (\varphi), \\
& \lambda_{2}=m-\sqrt{p}(\cos (\varphi)+\sqrt{3} \sin (\varphi)), \\
& \lambda_{3}=m-\sqrt{p}(\cos (\varphi)-\sqrt{3} \sin (\varphi)),
\end{aligned}
$$

y sus derivadas

$$
\begin{aligned}
& \frac{\partial \lambda_{1}}{\partial \theta_{i}}=\frac{\partial m}{\partial \theta_{i}}+p^{-1 / 2} \frac{\partial p}{\partial \theta_{i}} \cos (\varphi)-2 p^{1 / 2} \sin (\varphi) \frac{\partial \varphi}{\partial \theta_{i}} \\
& \frac{\partial \lambda_{2}}{\partial \theta_{i}}=\frac{\partial m}{\partial \theta_{i}}-\frac{1}{2} p^{-1 / 2} \frac{\partial p}{\partial \theta_{i}}(\cos (\varphi)+\sqrt{3} \sin (\varphi))-p^{1 / 2}(-\sin (\varphi)+\sqrt{3} \cos (\varphi)) \frac{\partial \varphi}{\partial \theta_{i}} \\
& \frac{\partial \lambda_{3}}{\partial \theta_{i}}=\frac{\partial m}{\partial \theta_{i}}-\frac{1}{2} p^{-1 / 2} \frac{\partial p}{\partial \theta_{i}}(\cos (\varphi)-\sqrt{3} \sin (\varphi))+p^{1 / 2}(\sin (\varphi)+\sqrt{3} \cos (\varphi)) \frac{\partial \varphi}{\partial \theta_{i}}
\end{aligned}
$$

Dado que

$$
\frac{\partial \varphi}{\partial \theta_{i}}=\frac{-1}{3 p\left(p^{3}-q^{2}\right)^{1 / 2}}\left(\frac{\partial q}{\partial \theta_{i}} p-\frac{3 q}{2} \frac{\partial p}{\partial \theta_{i}}\right)
$$

las derivadas de los autovalores quedan definidas tras el cálculo de $\frac{\partial m}{\partial \theta_{i}}, \frac{\partial p}{\partial \theta_{i}}$ y $\frac{\partial q}{\partial \theta_{i}}$. Es fácil mostrar

$$
\frac{\partial m}{\partial \theta_{i}}=\left\{\begin{array}{ll}
\frac{1}{3}, & \text { si } i=1,2,3 \\
0, & \text { en caso contrario }
\end{array} .\right.
$$

Tras tediosas cuentas se muestra también

$$
\frac{\partial p}{\partial \theta_{i}}=\left\{\begin{array}{ll}
\frac{1}{9}\left(2 \theta_{i}-\theta_{j}-\theta_{k}\right), & \text { si } i=1,2,3 \\
\frac{2}{3} \theta_{i}, & \text { si } i=4,5,6
\end{array},\right.
$$


en donde, en el primer caso se impone $i \neq j \neq k$ y $i, j, k \in\{1,2,3\}$. Finalmente $\frac{\partial q}{\partial \theta_{i}}=\left\{\begin{array}{ll}\frac{1}{6}\left[\frac{1}{3}\left(4 \theta_{j} \theta_{k}-2 \theta_{j} \theta_{i}-2 \theta_{i} \theta_{k}+2 \theta_{i}^{2}-\theta_{j}^{2}-\theta_{k}^{2}\right)-2 \theta_{l}^{2}+\theta_{m}^{2}+\theta_{n}^{2}\right], & \text { si } i=1,2,3 \\ \theta_{j} \theta_{k}-\frac{1}{3} \theta_{i}\left(2 \theta_{l}-\theta_{m}-\theta_{n}\right), & \text { si } i=4,5,6\end{array}\right.$, en donde, en ambos casos, $l=7-i$ y los índices son todos distintos entre sí. Asimismo, en el primer caso se pide $i, j, k \in\{1,2,3\}$ y $l, m, n \in\{4,5,6\}$, mientras que en el segundo se pide $l, m, n \in\{1,2,3\}$ y $i, j, k \in\{4,5,6\}$. 


\section{Bibliografía}

Afzali, M. y H. Soltanian-Zadeh. Comparison of voxel-based morphometry (VBM) and tractography of diffusion tensor MRI (DT-MRI) in temporal lobe epilepsy. En 18th Iranian Conference on Electrical Engineering (ICEE), págs. 18-23. Iran (2010).

Agirre-Arrizubieta, Z., G. Huiskamp, C. Ferrier, A. van Huffelen, y F. Leijten. Interictal magnetoencephalography and the irritative zone in the electrocorticogram. Brain, 132:3060-3071 (2009).

Aja-Fernández, S., A. Trisán-Vega, y P. C. de-la Higuera. DWI acquisition schemes and diffusion tensor estimation: A simulation-based study. En Conf. Proc. IEEE Eng. Med. Biol. Soc., págs. 3317-3320. Buenos Aires, Argentina (2010a).

Aja-Fernández, S., G. Vegas-Sánchez-Ferrero, y A. Tristán-Vega. About the background distribution in MR data: a local variance study. Magn. Reson. Imag., 28:739-752 (2010b).

Alexander, D. A general framework for experiment design in diffusion MRI and its application in measuring direct tissue-microstructure features. Magn. Reson. Med., 60:439-448 (1997).

American Psychiatric Association. Diagnostic and statistical manual of mental disorders (4th ed., text rev.). Amer Psychiatric Pub (2000).

Anderson, A. Theoretical analysis of the effects of noise on diffusion tensor imaging. Magn. Reson. Med., 46:1174-1188 (1990).

Assaf, Y. y P. Basser. Composite hindered and restricted model of diffusion (CHARMED) MR imaging of the human brain. Neuroimage, 27:48-58 (2005).

Awada, K. A., D. R. Jackson, S. B. Baumann, J. T. Williams, D. R. Wilton, P. Fink, y B. Prasky. Effects of conductivity uncertainties and modelling errors on EEG source localization using a 2-D model. IEEE Trans. Biomed. Eng., 45(9):1135-1145 (1998).

Baillet, S., J. J. Rivera, G. Marin, J. F. Mangin, J. Aubert, y L. Garnero. Evaluation of inverse methods and head models for EEG source localization using a human skull phantom. Phys. Med. Biol., 46:77-96 (2001). 
Ball, T., M. Kern, I. Mutschler, A. Aertsen, y A. Schulze-Bonhage. Signal quality of simultaneously recorded invasive and non-invasive EEG. Neuroimage, 46:708-716 (2009).

Bammer, R., M. Auer, S. Keeling, M. Augustin, L. Stables, R. Prokesch, R. Stollberger, M. Moseley, y F. Fazekas. Diffusion tensor imaging using single-shot SENSE-EPI. Magn. Reson. Med., 48:128-136 (2002).

Bammer, R., M. Augustin, S. Strasser-Fuchs, T. Seifert, P. Kapeller, R. Stollberger, F. Ebner, H. Hartung, y F. Fazekas. Magnetic resonance diffusion tensor imaging for characterizing diffuse and focal white matter abnormalities in multiple sclerosis. Magn. Reson. Med., 44:583-591 (2000).

Basser, P., J. Mattiello, y D. LeBihan. MR diffusion tensor spectroscopy and imaging. Biophys. J., 66:259-267 (1994).

Basser, P., S. Pajevic, C. Pierpaoli, J. Duda, y A. Aldroubi. In vivo fiber tractography using DT-MRI data. Magn. Reson. Med., 44:625-632 (2000).

Bast, T., T. Boppel, A. Rupp, I. Harting, K. Hoechstetter, S. Fauser, A. Schulze-Bonhage, D. Rating, y M. Scherg. Noninvasive source localization of interictal EEG spikes: effects of signal-tonoise ratio and averaging. J. Clin. Neurophysiol., 23(6):487-497 (2006).

Baumann, S., D. Wozny, S. Kelly, y F. Meno. The electrical conductivity of human cerebrospinal fluid at body temperature. IEEE Trans. Biomed. Eng., 44(3):220-223 (1997).

Becker, E. B., G. F. Carey, y J. T. Oden. Finite Elements: An Introduction (Volume I). PrenticeHall, Inc., Englewood Cliffs, New Jersey 07632 (1981).

Beltrachini, L., A. Blenkmann, N. von Ellenrieder, A. Petroni, H. Urquina, F. Manes, A. Ibáñez, y C. Muravchik. Impact of head models in N170 component localization: results in control subjects and ADHD patients. Actas del XVIII Congreso Argentino de Bioingeniería SABI, págs. 115-125 (2011a).

Beltrachini, L., A. Blenkmann, N. von Ellenrieder, A. Petroni, H. Urquina, F. Manes, A. Ibáñez, y C. Muravchik. Impact of head models in N170 component source imaging: results in control subjects and ADHD patients. J. Phys. Conf. Ser., 332:012019 (2011b).

Beltrachini, L., N. von Ellenrieder, y C. H. Muravchik. Análisis del problema directo en EEG sobre medios anisótropos: formulación matemática y simulación mediante FEM. Actas del XVII Congreso sobre Métodos Numéricos y sus Aplicaciones - ENIEF, págs. 3267-3282 (2008).

Beltrachini, L., N. von Ellenrieder, y C. H. Muravchik. Estimador regularizado de la matriz de covarianza y su aplicación en el análisis del problema inverso en EEG mediante beamforming. Actas de la XIII Reunión de Trabajo en Procesamiento de la Información y Control, RPIC 2009 , págs. 351-356 (2009). 
Beltrachini, L., N. von Ellenrieder, y C. H. Muravchik. Shrinkage approach for EEG covariance matrix estimation. Conf. Proc. IEEE Eng. Med. Biol. Soc., págs. 1654-1657 (2010).

Beltrachini, L., N. von Ellenrieder, y C. H. Muravchik. General bounds for electrode mislocation on the EEG inverse problem. Comput. Methods Programs. Biomed., 103(1):1-9 (2011c).

Beltrachini, L., N. von Ellenrieder, y C. H. Muravchik. A general framework for error analysis in multiple coil DTI estimation. Actas de la XIII Reunión de Trabajo en Procesamiento de la Información y Control, RPIC 2011, págs. 381-386 (2011d).

Beltrachini, L., N. von Ellenrieder, y C. H. Muravchik. Shrinkage approach for spatiotemporal EEG covariance matrix estimation (2011e). Enviado a IEEE Transactions on Signal Processing, Agosto de 2011.

Benar, C. y J. Gotman. Modeling of post-surgical brain and skull defects in the EEG inverse problem with the boundary element method. Clin. Neurophysiol., 113:48-56 (2002).

Bentin, S., Y. Mouchetant-Rostaing, M. Giard, J. Echallier, y J. Pernier. ERP manifestations of processing printed words at different psycholinguistic levels: Time course and scalp distribution. J. Cogn. Neurosci., 11(3):235-260 (1999).

Bijma, F., J. de Munck, y R. Heethaar. The spatiotemporal MEG covariance matrix modeled as a sum of Kronecker products. Neuroimage, 27:402-415 (2005).

Bijma, F., J. de Munck, H. Huizenga, y R. Heethaar. A mathematical approach to the temporal stationarity of background noise in MEG/EEG measurements. Neuroimage, 20:233-243 (2003).

Blenkmann, A., L. Beltrachini, N. von Ellenrieder, S. Kochen, y C. H. Muravchik. Evaluación de sLORETA para la resolución del problema inverso en ECoG utilizando modelos de poblaciones neuronales fisiológicamente plausibles. Actas de la XIV Reunión de Trabajo en Procesamiento de la Información y Control, RPIC 2011, págs. 120-125 (2011).

Blount, J., J. Cormier, H. Kim, P. Kankirawatana, K. Riley, y R. Knowlton. Advances in intracranial monitoring. Neurosurg. Focus., 25(3):E18 (2008).

Brebbia, C. A. y J. Domínguez. Boundary Elements. An Introductory Course. Computational Mechanics Publications. McGraw-Hill, segunda edición (1992).

Bruno, P., F. Vatta, y P. Inchingolo. Influence of EEG measurement montage on source localization error bounds due to head modeling errors caused by brain lesions. Conf. Proc. IEEE Eng. Med. Biol. Soc., págs. 825-828 (2004).

Bullmore, E. y O. Sporns. Complex brain networks: graph-theoretical analysis of structural and functional systems. Nat. Rev. Neurosci., 10:186-198 (2009).

Caan, M., H. Khedoe, D. Poot, A. den Dekker, S. Olabarriaga, K. Grimbergen, L. van Vliet, y 
F. Vos. Estimation of diffusion properties in crossing fiber bundles. IEEE Trans. Med. Imag., 29(8):1504-1515 (2010).

Cabral, B. y L. Leedom. Imaging vector fields using line integral convolution. Proc. SIGGRAPH'93 Conf., New York, USA, págs. 263-272 (1993).

Carey, G. F. y J. T. Oden. Finite Elements: A Second Course (Volume II). Prentice-Hall, Inc., Englewood Cliffs, New Jersey 07632 (1983).

Chang, L.-C., C. Koay, C. Pierpaoli, y P. Basser. Variance of estimated DTI-derived parameters via first-order perturbation methods. Magn. Reson. Med., 57:141-149 (2007).

Chen, Y., A. Wiesel, Y. Eldar, y A. Hero. Shrinkage algorithms for MMSE covariance estimation. IEEE Trans. Signal Process., 58(10):5016 -5029 (2010).

Chen, Y.-S., C.-Y. Cheng, J.-C. Hsieh, y L. Chen. Maximum contrast beamformer for electromagnetic mapping of brain activity. IEEE Trans. Biomed. Eng., 53(9):1765-1774 (2006).

Cho, J., S. Hong, Y. Jung, H. Kang, H. Kim, M. Suh, K. Jung, y C. Im. Evaluation of algorithms for intracranial EEG (iEEG) source imaging of extended sources: feasibility of using iEEG source imaging for localizing epileptogenic zones in secondary generalized epilepsy. Brain Topogr., 24(2):91-104 (2011).

Cobb, W. IFCN Recommendations For The Practice Of Clinical Neurophysiology. Elsevier, Amsterdam (1983).

Coleman, T. y Y. Li. On the convergence of reflective Newton methods for large-scale nonlinear minimization subject to bounds. Math. Programm., 67(2):189-224 (1994).

Coleman, T. y Y. Li. An interior trust region approach for nonlinear minimization subject to bounds. SIAM J. Optim., 6:418-445 (1996).

Constantinides, C., E. Atalar, y E. McVeigh. Signal-to-noise measurements in magnitude images from NMR phased arrays. Magn. Reson. Med., 38(5):852-857 (1997).

Corrigan, N., T. Richards, S. Webb, M. Murias, K. Merkle, N. Kleinhans, L. Johnson, A. Poliakov, E. Aylward, y G. Dawson. An investigation of the relationship between fMRI and ERP source localized measurements of brain activity during face processing. Brain Topogr., 22:83-96 (2009).

Cuffin, B. N. Effects of head shape on EEG's and MEG's. IEEE Trans. Biomed. Eng., 37(1):44-52 (1990).

Dale, A. M. y M. Sereno. Improved localization of cortical activity by combining EEG and MEG with MRI cortical surface reconstruction: a linear approach. J. Cogn. Neurosci., 5:162-176 (1993).

Dannhauer, M., B. Lanfer, C. Wolters, y T. Knösche. Modeling of the human skull in EEG source 
analysis. Human Brain Mapping (2010). In press.

de Jongh, A., J. de Munck, J. Baayen, E. Jonkman, R. Heethar, y B. V. Dijk. The localization of spontaneous brain activity: first results in patients with cerebral tumors. Clin. Neurophysiol., 112:378-385 (2001).

de Munck, J. The potential distribution in a layered anisotropic spheroidal volume conductor. $J$. Appl. Phys., 64:464-470 (1988).

de Munck, J. A linear discretization of the volume conductor boundary integral equation using analytically integrated elements. IEEE Trans. Biomed. Eng., 39(9):986-990 (1992).

de Munck, J., B. V. Dijk, y H. Spekreijse. Mathematical dipoles are adequate to describe realistic generators of human brain activity. IEEE Trans. Biomed. Eng., 35:960-966 (1988a).

de Munck, J., H. Huizenga, L. Waldorp, y R. Heethaar. Estimating stationary dipoles from MEG/EEG data contaminated with spatially and temporally correlated background noise. IEEE Trans. Signal Process., 50(7):1565-1572 (2002).

de Munck, J. y M. Peters. A fast method to compute surface potentials in the multisphere model. IEEE Trans. Biomed. Eng., 40(11):1166-1174 (1993).

de Munck, J., P. van Houdt, R. Verdaasdonk, y P. Ossenblok. A semi-automatic method to determine electrode positions and labels from gel artifacts in EEG/fMRI-studies. Neuroimage, 59(1):399-403 (2012).

de Munck, J., P. Vijn, y F. L. da Silva. A random dipole model for spontaneous brain activity. IEEE Trans. Biomed. Eng., 39(8):791-804 (1992).

de Munck, J., P. Vijn, y H. Spekreijse. A practical method for determining electrode positions on the head. Electroenceph. Clin. Neurophysiol., 78(1):85-87 (1991).

de Munck, J., P. Vijn, J. Verbunt, M. Faay, y B. van Dijk. A theoretical model for the spatial distribution of EEG/MEG for correlated and uncorrelated sources. Conf. Proc. IEEE Eng. Med. Biol. Soc., págs. 1228-1232 (1997).

de Munck, J. C., B. W. van Dijk, y H. Spekreijse. Mathematical dipoles are adequate to describe realistic generators of human brain activity. IEEE Trans. Biomed. Eng., 35(11):960-966 (1988b).

Deffke, I., T. Sander, J. Heidenreich, W. Sommer, G. Curio, y L. Trahms. MEG/EEG sources of the 170-ms response to faces are co-localized in the fusiform gyrus. Neuroimage, 35:1495-1501 (2007).

Deldin, P., J. Keller, J. Gergen, y G. Miller. Right-posterior face processing anomaly in depression. J. Abnorm. Psychol., 109:116-121 (2000).

Desikan, R., F. Se'gonne, B. Fischl, B. Quinn, B. Dickerson, D. Blacker, R. Buckner, A. Dale, 
R. Maguire, B. Hyman, M. Albert, y R. Killiany. An automated labeling system for subdividing the human cerebral cortex on MRI scans into gyral based regions of interest. Neuroimage, 31:968-980 (2006).

Deuschl, G. y A. Eisen. Recommendations for the practice of clinical neurophysiology: guidelines of the International Federation of Clinical Neurophysiology. Electroenceph. Clin. Neurophysiol., suppl. 52. Elsevier Science B. V., P. O. Box 211, 1000 AE Amsterdam, The Netherlands (2000).

Dietrich, O., J. Raya, S. Reeder, M. Ingrisch, M. Reiser, y S. Schoenberg. Influence of multi-channel combination, parallel imaging, and other reconstruction techniques on MRI noise characteristics. Magn. Reson. Imag., 26(6):754-762 (2008).

Dogandžić, A. y A. Nehorai. EEG/MEG spatio-temporal dipole source estimation and array design. IEEE Trans. Signal Process., 48(1):13-25 (2000).

Du, L., T. Yardibi, J. Li, y P. Stoica. Review of user parameter-free robust adaptive beamforming algorithms. Digital Signal Processing, 19(4):567-582 (2009).

Dümpelmann, M., T. Ball, y A. Schulze-Bonhage. sLORETA allows reliable distributed source reconstruction based on subdural strip and grid recordings. Hum. Brain Mapp. (2011). In press.

Dümpelmann, M., J. Fell, J. Wellmer, H. Urbach, y C. Elger. 3D source localization derived from subdural strip and grid electrodes: A simulation study. Clin. Neurophysiol., 120:1061-1069 (2009).

Engel, A., C. Moll, I. Fried, y G. Ojemann. Invasive recordings from the human brain: clinical insights and beyond. Nat. Rev. Neurosci., 6(1):35-47 (2011).

Ermer, J., J. Mosher, S. Baillet, y R. Leahy. Rapidly recomputable EEG forward models for realistic head shapes. Phys. Med. Biol., 46:1265-1281 (2001).

Fang, Q. y D. Boas. Tetrahedral mesh generation from volumetric binary and gray-scale images. Proc. IEEE Intl. Symp. Biomed. Imag., págs. 1142-1145 (2009).

Fernández-Corazza, M., L. Beltrachini, N. von Ellenrieder, y C. Muravchik. Tomografía de impedancia eléctrica y resonancia magnética como herramientas conjuntas para la estimación paramétrica de la conductividad eléctrica del cráneo y del cuero cabelludo. En Actas de la XIV Reunión de Trabajo Procesamiento de la Información y Control - RPIC 2011, págs. 845-850. Oro Verde, Argentina (2011).

Filippi, M., M. Cercignani, M. Inglese, M. Horsfield, y G. Comi. Diffusion tensor magnetic resonance imaging in multiple sclerosis. Neurology, 56:304-311 (2001).

Flemming, L., Y. Wang, A. Caprihan, M. Eiselt, J. Haueisen, y Y. Okada. Evaluation of the distortion of EEG signals caused by a hole in the skull mimicking the fontanel in the skull of human neonates. Clin. Neurophysiol., 116:1141-1152 (2005). 
Fountain, N., J. Kim, y S. Lee. Sleep deprivation activates epileptiform discharges independent of the activating effects of sleep. J. Clin. Neurophysiol., 15(1):69-75 (1998).

Freeman, W. Origin, structure, and role of background EEG activity. part 4. neural frame simulation. Clin. Neurophysiol., 117(3):572-589 (2005).

Friston, K., J. Ashburner, S. Kiebel, T. Nichols, y W. P. (eds). Statistical Parametric Mapping: The Analysis of Functional Brain Images. Academic Press (2007).

Fuchs, M., M. Wagner, H. Wischmann, T. Kohler, A. Theissen, R. Drenckhahn, y H. Buchner. Improving source reconstructions by combining bioelectric and biomagnetic data. Electroenceph. clin. Neurophysiol., 107:93-111 (1998).

Geselowitz, D. On bioelectric potentials in an inhomogenous volume conductor. Biophys. J., 7:1-11 (1967).

Geselowitz, D. On the magnetic field generated outside an inhomogeneous volume conductor by internal current sources. IEEE Trans. Magnetics, MAG-6:346-347 (1970).

Giannelli, M., G. Belmonte, N. Toschi, I. Pesaresi, P. Ghedin, A. Traino, C. Bartolozzi, y M. Cosottini. DTI measurements of fractional anisotropy and mean diffusivity at 1.5 T: Comparison of two radiofrequency head coils with different functional designs and sensitivities. Med. Phys., 38(6):3205-3211 (2011).

Gilbert, G., D. Simard, y G. Beaudoin. Impact of an improved combination of signals from array coils in diffusion tensor imaging. IEEE Trans. Med. Imaging, 26(11):1428-1436 (2007).

Goldenholz, D., S. Ahlfors, M. Hämäläinen, D. Sharon, M. Ishitobi, L. Vaina, y S. Stufflebeam. Mapping the Signal-To-Noise-Ratios of cortical sources in magnetoencephalography and electroencephalography. Hum. Brain Mapp., 30(4):1077-1086 (2009).

Gratton, G., M. Coles, y E. Donchin. A new method for off-line removal of ocular artifact. Electroenceph. Clin. Neurophysiol., 55:468-484 (1983).

Greenblatt, R., A. Ossadtchi, y M. Pflieger. Local linear estimators for the bioelectromagnetic inverse problem. IEEE Trans. Signal Process., 53(9):3403-3412 (2005).

Griswold, M., P. Jakob, R. Heidemann, M. Nittka, V. Jellus, J. Wang, B. Kiefer, y A. Haase. Generalized autocalibrating partially parallel acquisitions (GRAPPA). Magn. Reson. Med., 47:1202-1210 (2002).

Grossmann, T. The development of emotion perception in face and voice during infancy. Restorative Neurology and Neuroscience, 28:219-236 (2010).

Gu, Y. y A. Leshem. Robust adaptive beamforming based on jointly estimating covariance matrix and steering vector. En IEEE Intl. Conf. Acoustics, Speech and Signal Process (ICASSP), págs. 
2640-2643. Prague, Czech Republic (2011).

Gudbjartsson, H. y S. Patz. The rician distribution of noisy MRI data. Magn. Reson. Med., 34:910-914 (1995).

Güllmar, D., J. Haueisen, y J. Reichenbach. Influence of anisotropic electrical conductivity in white matter tissue on the EEG/MEG forward and inverse solution. a high-resolution whole head simulation study. Neuroimage, 51:145-163 (2010).

Haalman, I. y E. Vaadia. Dynamics of neuronal interactions: Relation to behavior, firing rates, and distance between neurons. Hum. Brain Mapp., 5(4):249-253 (1997).

Hagmann, P., J. Thiran, L. Jonasson, P. Vandergheynst, S. Clarke, P. Maeder, y R. Meuli. DTI mapping of human brain connectivity: statistical fibre tracking and virtual dissection. Neuroimage, 19(3):545-554 (2003).

Hallez, H., B. Vanrumste, R. Grech, J. Muscat, W. De Clercq, A. Vergult, Y. D'Asseler, K. Camilleri, S. Fabri, S. Van Huffel, y I. Lemahieu. Review on solving the forward problem in EEG source analysis. J. Neuroeng. Rehabil., 46 (2007).

Hämäläinen, M., R. Hari, R. Ilmoniemi, J. Knuutila, y O. Lounasmaa. Magnetoencephalographytheory, instrumentation, and applications to noninvasive studies of the working human brain. Rev. Mod. Phys., 65(2):413-497 (1993).

Harris, A., B. Duchaine, y K. Nakayama. Normal and abnormal face selectivity of the M170 response in developmental prosopagnosics. Neuropsychologia, 43:2125-2136 (2005).

Hasan, K. A framework for quality control and parameter optimization in diffusion tensor imaging: Theoretical analysis and validation. Magn. Reson. Imaging, 25(8):1196-1202 (2007).

Hasan, K., A. Alexander, y P. Narayana. Does fractional anisotropy have better noise immunity characteristics than relative anisotropy in diffusion tensor MR? an analytical approach. Magn. Reson. Med., 51:413-417 (2004).

Hasan, K., P. Basser, D. Parker, y A. Alexander. Analytical computation of the eigenvalues and eigenvectors in DT-MRI. J. Magn. Reson., 152:41-47 (2001).

Hashiguchi, K., T. Morioka, F. Yoshida, Y. Miyagi, S. Nagata, A. Sakata, y T. Sasaki. Correlation between scalp-recorded electroencephalographic and electrocorticographic activities during ictal period. Seizure, 16:238-247 (2007).

Hastie, T., R. Tibshirani, y J. Friedman. The Elements of Statistical Learning. Springer, New York (2001).

Haueisen, J., A. Büttner, M. Funke, H. Brauer, y H. Nowak. Influence of boundary element discretization on the forward and inverse problem in electroencephalography and magnetoen- 
cephalography. Biomedizinische Technik, 42:240-248 (1997).

Hayes, L., B. Sun, R. Jones, R. Brown, y T. Burns. Occult white matter diffusion abnormalities detected by tract-based spatial statistics (TBSS) in children with sickle cell anemia. En 6th Congress and Exhibition of the joint Societies of Paediatric Radiology (IPR). London, UK (2011).

Herrmann, M., A.-C. Ehlis, A. Mühlberger, y A. Fallgatter. Source localization of early stages of face processing. Brain Topogr., 18(2):77-85 (2005).

Herrmann, M., T. Schreppel, S. Biehl, C. Jacob, M. Heine, A. Boreatti-Hümmer, A. Mühlberger, y A. Fallgatter. Emotional deficits in adult ADHD patients: an ERP study. Soc. Cogn. Affect. Neurosci., 4:340-345 (2009).

Hochwald, B. y A. Nehorai. Magnetoencephalography with diversely oriented and multi component sensors. IEEE Trans. Biomed. Eng., 44:40-50 (1997).

Hoey, G. V., B. Vanrumste, M. D'Havé, R. V. de Walle, I. Lemahieu, y P. Boon. Influence of measurement noise and electrode mislocalisation on EEG dipole-source localisation. Med. Biol. Eng. Comput., 38(3):287-296 (2000).

Horn, R. y C. Johnson. Matrix Analysis. Cambridge, U.K.: Cambridge Univ. Press (1985).

Huang, C.-C. y J.-H. Lee. Robust adaptive beamforming using a fully data-dependent loading technique. PIER B, 37:307-325 (2012).

Huiskamp, G., Z. Agirre-Arrizubieta, y F. Leijten. Regional differences in the sensitivity of MEG for interictal spikes in epilepsy. Brain Topogr., 23:159-164 (2010).

Huiskamp, G., M. Vroeijenstijn, R. van Dijk, G. Wieneke, y A. van Huffelen. The need for correct realistic geometry in the inverse EEG problem. IEEE Trans. Biomed. Eng., 46(11):1281-1287 (1999).

Huizenga, H., J. de Munck, L. Waldorp, y R. Grasman. Spatiotemporal EEG/MEG source analysis based on a parametric noise covariance model. IEEE Trans. Biomed. Eng., 49(6):533-539 (2002).

Hutton, D. V. Fundamentals of Finite Element Analysis. The McGraw-Hill Companies (2004).

Ibáñez, A., E. Hurtado, R. Riveros, H. Urquina, J. Cardona, A. Petroni, A. Lobos-Infante, J. Barutta, S. Baez, y F. Manes. Facial and semantic emotional interference: a pilot study on the behavioral and cortical responses to the dual valence association task. Behav. Brain Funct., 13:8 (2011a).

Ibáñez, A., A. Petroni, H. Urquina, F. Torrente, T. Torralva, E. Hurtado, R. Guex, A. Blenkmann, L. Beltrachini, C. Muravchik, S. Baez, M. Cetckovich, M. Sigman, A. Lischinsky, y F. Manes. Cortical deficits of emotional face processing in adults with ADHD: its relation to social cognition 
and executive function. Soc. Neurosci., 6(5-6):464-481 (2011b).

Ibáñez, A., H. Urquina, A. Petroni, S. Baez, M. do Nascimento, R. Guex, E. Hurtado, A. Blenkmann, L. Beltrachini, M. Sigman, A. Lischinsky, T. Torralva, F. Torrente, M. Cetkovich, y F. Manes. Abnormal neural processing of emotional facial and semantic expressions in adults with euthymic bipolar disorder and their relation to clinical, cognitive and social cognition profile (2011c). Enviado a J. Affect. Disord.

Itier, R. y M. Taylor. Source analysis of the N170 to faces and objects. Neuroreport, 15(8):12611265 (2004).

Iwasaki, M., E. Pestana, R. Burgess, H. Lüders, H. Shamoto, y N. Nakasato. Detection of epileptiform activity by human interpreters: blinded comparison between electroencephalography and magnetoencephalography. Epilepsia, 46(1):59-68 (2005).

Jackson, J. D. Classical Electrodynamics. John Wiley \& Sons, segunda edición (1975).

Jaermann, T., G. Crelier, K. Pruessmann, X. Golay, T. Netsch, A. van Muiswinkel, S. Mori, P. van Zijl, A. Valavanis, S. Kollias, y P. Boesiger. SENSE-DTI at 3T. Magn. Reson. Med., 51:230-236 (2004).

Jaermann, T., N. D. Zanche, P. Staempfli, K. Pruessmann, A. Valavanis, P. Boesiger, y S. Kollias. Preliminary experience with visualization of intracortical fibers by focused high-resolution diffusion tensor imaging. AJNR Am. J. Neuroradiol., 29:146-150 (2008).

James, W. y J. Stein. Estimation with quadratic loss. Proc. Third. Berkeley Symp. Math. Statist. Probab., págs. 361-379 (1961).

Jenkinson, M., M. Pechaud, y S. Smith. BET2: MR-based estimation of brain, skull and scalp surfaces. Ann. Meeting Org. Hum. Brain Mapp. (2005).

Jeong, H.-K. y A. Anderson. Characterizing fiber directional uncertainty in diffusion tensor MRI. Magn. Reson. Med., 60(6):1408-1421 (2008).

Johnson, C. Numerical solutions of partial differential equations by the finite element method. Cambridge University Press, Cambridge (1987).

Jones, D. Determining and visualization uncertainty in estimates of fiber orientation from diffusion tensor MRI. Magn. Reson. Med., 49:7-12 (2003).

Jones, D. The effect of gradient sampling schemes on measures derived from diffusion tensor MRI: A Monte Carlo study. Magn. Reson. Med., 51:807-815 (2004).

Jones, D., M. Horsfield, y A. Simmons. Optimal strategies for measuring diffusion in anisotropic systems by magnetic resonance imaging. Magn. Reson. Med., 42:515-525 (1999).

Kang, X., T. Herron, y D. Woods. Validation of the anisotropy index ellipsoidal area ratio in 
diffusion tensor imaging. Magn. Reson. Imag., 28:546-556 (2010).

Kavanagh, R., T. Darcey, D. Lehmann, y D. Fender. Evaluation of methods for three-dimensional localization of electrical sources in the human brain. IEEE Trans. Biomed. Eng., 25(5):421-429 (1978).

Kay, S. Fundamentals of Statistical Signal Processing: Estimation Theory. Signal Processing Series. Prentice-Hall (1993).

Khatri, C. y C. Rao. Effects of estimated noise covariance matrix in optimal signal detection. IEEE Trans. Acoust., Speech, Signal Process., (5):671-679 (1987).

Khosla, D., M. Don, y B. Kwong. Spatial mislocation of EEG electrodes - effects on accuracy of dipole estimation. Clin. Neurophysiol., 110(2):261-271 (1999).

Kim, J., C. Im, Y. Jung, E. Kim, S. Lee, y C. Chung. Localization and propagation analysis of ictal source rhythm by electrocorticography. Neuroimage, 52(4):1279-1288 (2010).

Kim, S., T. Kim, Y. Zhou, y M. Singh. Influence of conductivity tensors on the scalp electrical potential: Study with 2-d finite element models. IEEE Trans. Nuclear Science, 50:133-139 (2003).

Koay, C., L.-C. Chang, C. Pierpaoli, y P. Basser. Error propagation framework for diffusion tensor imaging via diffusion tensor representations. IEEE Trans. Med. Imaging, 26(8):10171034 (2007).

Koay, C., S. Hurley, y M. Meyerand. Extremely efficient and deterministic approach to generating optimal ordering of diffusion MRI measurements. Med. Phys., 38(8):4795-4801 (2011).

Koay, C., U. Nevo, L.-C. Chang, C. Pierpaoli, y P. Basser. The elliptical cone of uncertainty and its normalized measures in diffusion tensor imaging. IEEE Trans. Med. Imaging, 27(6):834-846 (2008).

Kobayashi, K., Y. Harumi, O. Yoko, y J. Gotman. Dipole modeling of epileptic spikes can be accurate or misleading. Epilepsia, 46:397-408 (2005).

Koch, M., D. Norris, y M. Hund-Georgiadis. An investigation of functional and anatomical connectivity using magnetic resonance imaging. Neuroimage, 16(1):241-250 (2002).

Koenig, T., K. Kochi, y D. Lehmann. Event-related electric microstates of the brain differ between words with visual and abstract meaning. Electroencephalogr. Clin. Neurophysiol., 106(6):535546 (1998).

Kollo, T. y D. von Rosen. Advanced Multivariate Statistics with Matrices. Springer (2005).

Korzyukov, O., M. Pflieger, M. Wagner, S. Bowyer, T. Rosburg, K. Sundaresan, C. Elger, y N. Boutros. Generators of the intracranial P50 response in auditory sensory gating. Neuroimage, 
$35(2): 814-826(2007)$.

Lachaux, J., D. Rudrauf, y P. Kahane. Intracranial EEG and human brain mapping. J. Physiol. Paris, 97:613-628 (2003).

Landman, B., P.-L. Bazin, y J. Prince. Diffusion tensor estimation by maximizing Rician likelihood. En Int. Conf. of the IEEE on Computer Vision (ICCV). Rio de Janeiro, Brazil (2007).

Landman, B., P.-L. Bazin, y J. Prince. Estimation and application of spatially variable noise fields in diffusion tensor imaging. Magn. Reson. Imaging, 27(6):741-751 (2009).

Lantz, G., R. G. de Peralta, L. Spinelli, M. Seeck, y C. Michel. Epileptic source localization with high density EEG: how many electrodes are needed? Clinical Neurophysiology, 114:63-69 (2003).

Larkman, D. y R. Nunes. Parallel magnetic resonance imaging. Phys. Med. Biol., 52:R15-R55 (2007).

Larsson, E., D. Erdogmus, R. Yan, J. Principe, y J. Fitzsimmons. SNR-optimality of sum-of-squares reconstruction for phased-array magnetic resonance imaging. J. Magn. Reson., 163:121-123 (2003).

Lazar, M. y A. Alexander. An error analysis of white matter tractography methods: synthetic diffusion tensor field simulations. Neuroimage, 20:1140-1153 (2003).

Lazar, M., D. Weinstein, J. Tsuruda, K. Hasan, K. Arfanakis, M. Meyerand, B. Badie, H. Rowley, V. Haughton, A. Field, y A. Alexander. White matter tractography using diffusion tensor deflection. Hum. Brain Mapp., 18:306-321 (2003).

LeBihan, D. Molecular diffusion nuclear magnetic resonance imaging. Magn. Reson. Q., 7:1-30 (1991).

Ledoit, O. y M. Wolf. A well-conditioned estimator for large-dimensional covariance matrices. $J$. Multiv. Anal., 88:365-411 (2001).

Li, J., L. Du, y P. Stoica. Fully automatic computation of diagonal loading levels for robust adaptive beamforming. IEEE Int. Conf. Acoust., Speech, Signal Processing (ICASSP), págs. 2325 -2328 (2008). ISSN 1520-6149.

Li, J., K. Wang, S. Zhu, y B. He. Effects of holes on EEG forward solutions using a realistic geometry head model. J. Neural Eng., 4:197-204 (2007).

Liu, Z., M. Fukunaga, J. de Zwart, y J. Duyn. Large-scale spontaneous fluctuations and correlations in brain electrical activity observed with magnetoencephalography. Neuroimage, 51:102-111 (2010).

Lütkenhöner, B. Dipole source localization by means of maximum likelihood estimation. I. theory and simulations. Clin. Neurophysiol., 106:314-321 (1998). 
Maier, S., P. Bogner, G. Bajzik, H. Mamata, Y. Mamata, I. Repa, F. Jolesz, y R. Mulkern. Normal brain and brain tumor: Multicomponent apparent diffusion coefficient line scan imaging. Radiology, 219:842-849 (2001).

Maier, S., S. Vajapeyam, H. Mamata, C.-F. Westin, F. Jolesz, y R. Mulkern. Biexponential diffusion tensor analysis of human brain diffusion data. Magn. Reson. Med., 51(2):321-330 (2004).

Mallik, R. On multivariate Rayleigh and Exponential distributions. IEEE Trans. Inf. Theory, 49(6):1499-1515 (2003).

Malmivuo, J. y R. Plonsey. Bioelectromagnetism - Principles and Applications of Bioelectric and Biomagnetic Fields. Oxford University Press (1995).

Malow, B., L. Selwa, D. Ross, y M. Aldrich. Lateralizing value of interictal spikes on overnight sleep-EEG studies in temporal lobe epilepsy. Epilepsia, 40(11):1587-1592 (1999).

Mang, S., D. Gembris, W. Grodd, y U. Klose. Comparison of gradient encoding directions for higher order tensor diffusion data. Magn. Reson. Med., 61(2):335-343 (2009).

Marsh, P. y L. Williams. ADHD and schizophrenia phenomenology: visual scanpaths to emotional faces as a potential psychophysiological marker? Neurosci. Biobehav. Rev., 30:651-665 (2006).

Mazziotta, J., A. Toga, A. Evans, P. Fox, J. Lancaster, K. Zilles, R. Woods, T. Paus, G. Simpson, B. Pike, C. Holmes, L. Collins, P. Thompson, D. MacDonald, M. Iacoboni, T. Schormann, K. Amunts, N. Palomero-Gallagher, S. Geyer, L. Parsons, K. Narr, N. Kabani, G. L. Goualher, D. Boomsma, T. Cannon, R. Kawashima, y B. Mazoyer. A probabilistic atlas and reference system for the human brain: International consortium for brain mapping (ICBM). Philos. Trans. R. Soc. Lond. B Biol. Sci., 356:1293-1322 (2001).

Meijs, J., O. Weier, M. Peters, y A. van Oosterom. On the numerical accuracy of the boundary element method. IEEE Trans. Biomed. Eng., 36:1038-1049 (1989).

Meijs, J. W. H., B. J. ten Voorde, M. J. Peters, C. J. Stock, y F. H. L. da Silva. On the influence of various head models on EEG's and MEG's. En Pfurtscheller, G. y F. H. L. da Silva, eds., Functional Brain Imaging. Springer Verlag, Berlin (1988).

Merlet, I. y J. Gotman. Reliability of dipole models of epileptic spikes. Clin. Neurophysiol., 110:1013-1028 (1999).

Michel, C., T. Koenig, D. Brandeis, L. Gianotti, y J. Wackermann. Electrical Neuroimaging. Cambridge University Press (2009).

Mikuni, N., T. Nagamine, A. Ikeda, K. Terada, W. Taki, J. Kimura, y H. K. anbd H. Shibasaki. Simultaneous recording of epileptiform discharges by MEG and subdural electrodes in temporal lobe epilepsy. Neuroimage, 5:298-306 (1997). 
Miller, K., M. denNijs, P. Shenoy, J. Miller, R. Rao, y J. Ojemann. Real-time functional brain mapping using electrocorticography. Neuroimage, 37(2):504-507 (2007).

Mnatsakanian, E. y I. Tarkka. Familiar-face recognition and comparison: source analysis of scalprecorded event-related potentials. Clin. Neurophysiol., 115:880-886 (2004).

Mori, S., B. Crain, V. Chacko, y P. van Zijl. Three dimensional tracking of axonal projections in the brain by magnetic resonance imaging. Ann. Neurol., 45(2):265-269 (1999).

Mori, S., K. Oishi, H. Jiang, L. Jiang, X. Li, K. Akhter, K. Hua, A. Faria, A. Mahmood, R. Woods, A. Toga, G. Pike, P. R. Neto, A. Evans, J. Zhang, H. Huang, M. Miller, P. van Zijl, y J. Mazziotta. Stereotaxic white matter atlas based on diffusion tensor imaging in an ICBM template. Neuroimage, 40:570-582 (2008).

Moseley, M., Y. Cohen, J. Kucharczyk, J. Mintorovitch, H. Asgari, M. Wendland, J. Tsuruda, y D. Norman. Diffusion-weighted MR imaging of anisotropic water diffusion in cat central nervous system. Radiology, 176(2):439-445 (1990).

Mosher, J., R. Leahy, y P. Lewis. EEG and MEG: Forward solutions for inverse methods. IEEE Trans. Biomed. Eng., 46(3):245-259 (1999).

Mosher, J. C. y R. M. Leahy. Recursive MUSIC: A framework for EEG and MEG source localization. IEEE Trans. Biomed. Eng., 45(11):1342-1354 (1998).

Mosher, J. C., M. E. Spencer, R. M. Leahy, y P. S. Lewis. Error bounds for MEG and EEG dipole source localization. Electroencephalogr. Clin. Neurophysiol., 86:303-321 (1993).

Mukherjee, P., S. Chung, J. Berman, C. Hess, y R. Henry. Diffusion tensor MR imaging and fiber tractography: technical considerations. AJNR Am. J. Neuroradiol., 29(5):843-852 (2008).

Muravchik, C. y A. Nehorai. EEG/MEG error bounds for a static dipole source with a realistic head model. IEEE Trans. Signal Process., 49(3):470-484 (2001).

Niethammer, M., R. Estepar, S. Bouix, M. Shenton, y C.-F. Westin. On diffusion tensor estimation. En Conf. Proc. IEEE Eng. Med. Biol. Soc., págs. 2622-2625. New York, USA (2006).

Oishi, M., H. Otsubo, S. Kameyama, N. Morota, H. Masuda, M. Kitayama, y R. Tanaka. Epileptic spikes: Magnetoencephalography versus simultaneous electrocorticography. Epilepsia, 43(11):1390-1395 (2002).

Olbrich, S., C. Mulert, S. Karch, M. Trenner, G. Leicht, O. Pogarell, y U. Hegerl. EEG-vigilance and BOLD effect during simultaneous EEG/fMRI measurement. Neuroimage, 45:319-332 (2009).

Ollikainen, J., M. Vauhkonen, P. Karjalainen, y J. Kaipio. Effects of local skull inhomogeneities on EEG source estimation. Med. Eng. Phys., 21:143-154 (1999).

Ollikainen, J., M. Vauhkonen, P. A. Karjalainen, y J. P. Kaipio. Effects of electrode properties on 
EEG measurements and a related inverse problem. Med. Eng. Phys., 22:535-545 (2000).

Oostendorp, T., J. Delbecke, y D. Stegeman. The conductivity of the human skull: Results of in vivo and in vitro measurements. IEEE Trans. Biomed. Eng., 47(11):1467-1492 (2000).

O'Gorman, R. y D. Jones. Just how much data need to be collected for reliable bootstrap DT-MRI? Magn. Reson. Med., 56:884-890 (2006).

Pacia, S. y J. Ebersole. Intracranial EEG substrates of scalp ictal patterns from temporal lobe foci. Epilepsia, 38(6):642-654 (1997).

Pajevic, S. y P. Basser. Parametric and non-parametric statistical analysis of DT-MRI data. J. Magn. Reson., 161:1-14 (2003).

Papadakis, N., D. Xing, G. Houston, J. Smith, M. Smith, M. James, A. Parsons, C. Huang, L. Hall, y T. Carpenter. A study of rotationally invariant and symmetric indices of diffusion anisotropy. Magn. Reson. Imaging, 17(6):881-892 (1999).

Pascual-Marqui, R. Standardized low resolution brain electromagnetic tomography (sLORETA): technical details. Methods Find. Exp. Clin. Pharmacol., 24:5-12 (2002).

Peeters, T., P. Rodrigues, A. Vilanova, y B. ter Haar Romeny. Analysis of Distance/Similarity Measures for Diffusion Tensor Imaging, in Visualization and Processing of Tensor Fields. Advances and perspectives. D. Laidlaw and J. Weickert, ed.. Springer (2009).

Persson, P. O. y G. Strang. A simple mesh generator in MATLAB. SIAM Review, 46(2):329-345 (2004).

Pierpaoli, C., P. Jezzard, P. Basser, A. Barnett, y G. D. Chiro. Diffusion tensor MR imaging of the human brain. Radiology, 201(3):637-648 (1996).

Poepel, A., C. Helmstaedter, E. Kockelmann, N. Axmacher, W. Burr, C. Elger, y J. Fell. Correlation between EEG rhythms during sleep: surface versus mediotemporal EEG. Neuroreport, 18(8):837-840 (2007).

Poonawalla, A. y X. Zhou. Analytical error propagation in diffusion anisotropy calculations. J. Magn. Reson. Imag., 19:489-498 (2004).

Poot, D., A. den Dekker, E. Achten, M. Verhoye, y J. Sijbers. Optimal experimental design for diffusion kurtosis imaging. IEEE Trans. Med. Imaging, 29(3):819-829 (2010).

Porat, B. A Course in Digitial Signal Processing. John Wiley and Sons (1996).

Pruessmann, K., M. Weiger, M. Scheidegger, y P. Boesiger. SENSE: Sensitivity encoding for fast MRI. Magn. Reson. Med., 44:952-962 (1999).

Radich, B. M. y K. M. Buckley. EEG dipole localization bounds and MAP algorithms for head 
models with parameter uncertainties. IEEE Trans. Signal Process., 42:233-241 (1995).

Ramon, C., P. Schimpf, y J. Haueisen. Influence of head models on EEG simulations and inverse source localizations. BioMed Eng OnLine, 5:10 (2006).

Ray, A., J. Tao, S. Hawes-Ebersole, y J. Ebersole. Localizing value of scalp EEG spikes: A simultaneous scalp and intracranial study. Clin. Neurophysiol., 118:69-79 (2007).

Robinson, S. y J. Vrba. Functional neuroimaging by synthetic aperture magnetometry (SAM). Recent Advances in Biomagnetism. Sendai, Japan, Tohoku Univ. Press, págs. 302-305 (1999).

Roemer, P., W. Edelstein, C. Hayes, S. Souza, y O. Mueller. The NMR phased array. Magn. Reson. Med., 16:192-225 (1990).

Rossion, B. y C. Jacques. Does physical interstimulus variance account for early electrophysiological face sensitive responses in the human brain? ten lessons on the N170. Neuroimage, 39:1959-1979 (2008).

Rossion, B., C. Joyce, G. Cottrell, y M. Tarr. Early lateralization and orientation tuning for face, word, and object processing in the visual cortex. Neuroimage, 20(3):1609-1624 (2003).

Roth, B., A. Gorbach, y S. Sato. How well does a three-shell model predict positions of dipoles in a realistically shaped head? Electroenceph. Clin. Neurophysiol., 87:175-184 (1993).

Rullmann, M., A. Anwander, M. Dannhauer, S. Warfield, F. Duffy, y C. Wolters. EEG source analysis of epileptiform activity using a $1 \mathrm{~mm}$ anisotropic hexahedra finite element head model. Neuroimage, 44:399-410 (2009).

San Martín, J. P. Determinacion de la posicion de electrodos de electroencefalografia sobre la cabeza humana. Trabajo final de Carrera. Facultad de Ingeniería, Universidad Nacional de La Plata, Argentina (2006).

Sancetta, A. Sample covariance shrinkage for high dimensional dependent data. J. Multivar. Anal., 99(5):949-967 (2008).

Santesso, D., A. Meuret, S. Hofmann, E. Mueller, K. Ratner, E. Roesch, y D. Pizzagalli1. Electrophysiological correlates of spatial orienting towards angry faces: A source localization study. Neuropsychologia, 46(5):1338-1348 (2008).

Santiuste, M., R. Nowak, A. Russi, T. Tarancon, B. Oliver, E. Ayats, G. Scheler, y G. Graetz. Simultaneous magnetoencephalography and intracranial eeg registration: technical and clinical aspects. J. Clin. Neurophysiol., 25(6):331-339 (2008).

Sarvas, J. Basic mathematical and electromagnetic concepts of the biomagnetic inverse problem. Phys. Med. Biol., 32:11-22 (1987).

Schäfer, J. y K. Strimmer. A shrinkage approach to large-scale covariance matrix estimation and 
implications for functional genomics. Stat. Appl. Genet. Mol. Biol., 4(1) (2005).

Schimpf, P., C. Ramon, y J. Haueisen. Dipole models for the EEG and MEG. IEEE Trans. Biomed. Eng., 49(5):409-418 (2002).

Schlitt, H. A., L. Heller, R. Aaron, E. Best, y D. M. Ranken. Evaluation of boundary element methods for the EEG problem: Effect of linear interpolation. IEEE Trans. Biomed. Eng., $42(1): 52-58(1995)$.

Schweinberger, S., E. Pickering, I. Jentzsch, A. Burton, y J. Kaufmann. Event-related brain potential evidence for a response of inferior temporal cortex to familiar face repetitions. Cogn. Brain Res., 14:398-409 (2002).

Sekihara, K. y S. Nagarajan. Adaptive Spatial Filters for Electromagnetic Brain Imaging. Springer, Berlin (2007).

Sekihara, K., S. Nagarajan, D. Poeppel, y A. Marantz. Asymptotic SNR of scalar and vector minimum-variance beamformers for neuromagnetic source reconstruction. IEEE Trans. Biomed. Eng., 51(10):1726-1734 (2004).

Sekihara, K., S. Nagarajan, D. Poeppel, A. Marantz, y Y. Miyashita. Reconstructing spatiotemporal activities of neural sources using an MEG vector beamformer technique. IEEE Trans. Biomed. Eng., 48:760-771 (2001).

Sekihara, K., S. Nagarajan, D. Poeppel, y Y. Miyashita. Time-frequency MEG-MUSIC algorithm. IEEE Trans. Med. Imag., 18(1):92-97 (1999).

Sekihara, K., M. Sahani, y S. Nagarajan. Localization bias and spatial resolution of adaptive and non-adaptive spatial filters for MEG source reconstruction. Neuroimage, 25:1056-1067 (2005).

Sekihara, K., F. Takeuchi, S. Kuriki, y H. Koizumi. Reduction of brain noise influence in evoked neuromagnetic source localization using noise spatial correlation. Phys. Med. Biol., 39:937-946 (1994).

Shenoy, P., K. Miller, J. Ojemann, y R. Rao. Generalized features for electrocorticographic BCIs. IEEE Trans Biomed Eng., 55(1):273-280 (2008).

Shibata, T., H. Nishijo, R. Tamura, K. Miyamoto, S. Eifuku, S. Endo, y T. Ono. Generators of visual evoked potentials for faces and eyes in the human brain as determined by dipole localization. Brain Topogr., 15(1):51-63 (2002).

Shigeto, H., T. Morioka, K. Hisada, S. Nishio, H. Ishibashi, D. Kira, S. Tobimatsu, y M. Kato. Feasibility and limitations of magnetoencephalographic detection of epileptic discharges: simultaneous recording of magnetic fields and electrocorticography. Neurol. Res., 24(6):531-536 (2002). 
Silvester, P. y R. Ferrari. Finite Elements for Electrical Engineers. Cambridge University Press, Cambridge, second edición (1994).

Skare, S. Optimisation strategies in diffusion tensor MR imaging. Tesis Doctoral, MR Center, Dept. of Clinical Neuroscience, Karolinska Institutet, Sweeden (2002).

Skare, S., M. Hedehus, M. Moseley, y T.-Q. Li. Condition number as a measure of noise performance of diffusion tensor data acquisition schemes with MRI. J. Magn. Reson., 147:340-352 (2000).

Smith, O. Eigenvalues of a symmetric $3 \times 3$ matrix. Commun. ACM, 4(4):168 (1961).

Smith, S. Covariance, subspace, and intrinsic Cramér-Rao bounds. IEEE Trans. Sign. Process., 53(5):1610-1630 (2005).

Sokhadze, E., A. Tasman, R. Tamas, y R. El-Mallakh. Event-related potential study of the effects of emotional facial expressions on task performance in euthymic bipolar patients. Appl. Psychophysiol. Biofeedback, 1:1-13 (2010).

Sorensen, A., O. Wu, W. Copen, T. Davis, R. Gonzalez, W. Koroshetz, T. Reese, B. Rosen, V. Wedeen, y R. Weisskoff. Human acute cerebral ischemia: detection of changes in water diffusion anisotropy by using MR imaging. Radiology, 212:785-792 (1999).

Sprengelmeyer, R. y I. Jentzsch. Event related potentials and the perception of intensity in facial expressions. Neuropsychologia, 44:2899-2906 (2006).

Springer, C. Volume coordinates. Amer. Math. Monthly, 53(7):377-383 (1946).

Stein, C. Inadmissibility of the usual estimator for the mean of a multivariate normal distribution. Proc. Third. Berkeley Symp. Math. Statist. Probab., 1:197-206 (1956).

Stejskal, E. y J. Tanner. Spin diffusion measurements: Spin echoes in the presence of a timedependent field gradient. J. Chem. Phys., 42(1):288-292 (1965).

Stirnberg, R., T. Stöcker, y N. Shah. A new and versatile gradient encoding scheme for DTI: a direct comparison with the Jones scheme. En Proc. Intl. Soc. Mag. Reson. Med., pág. 3574. Honolulu, USA (2009).

Stoica, P., J. Li, X. Zhu, y J. Guerci. On using a priori knowledge in space-time adaptive processing. IEEE Trans. Signal Process., 56:2598-2602 (2008).

Sumiyoshi, T., Y. Higuchi, T. Itoh, M. Matsui, H. Arai, M. Suzuki, M. Kurachi, C. Sumiyoshi, y Y. Kawasaki. Effect of perospirone on P300 electrophysiological activity and social cognition in schizophrenia: A three-dimensional analysis with sLORETA. Psych. Res. Neuroimag., 172:180$183(2009)$.

Sutherling, W., M. Akhtari, A. Mamelak, J. Mosher, D. Arthur, S. Sands, P. Weiss, N. Lopez, M. DiMauro, E. Flynn, y R. Leah. Dipole localization of human induced focal afterdischar- 
ge seizure in simultaneous magnetoencephalography and electrocorticography. Brain Topogr., 14(2):101-116 (2001).

Tan, C. The EEG and epilepsy. Sing. Med. J., 30:424-425 (1989).

Tanaka, N., M. Hämäläinen, S. Ahlfors, H. Liu, J. Madsen, B. Bourgeois, J. Lee, B. Dworetzky, J. Belliveau, y S. Stufflebeam. Propagation of epileptic spikes reconstructed from spatiotemporal magnetoencephalographic and electroencephalographic source analysis. Neuroimage, 50(1):217$222(2010)$.

Tao, J., M. Baldwin, S. Hawes-Ebersole, y J. Ebersole. Cortical substrates of scalp EEG epileptiform discharges. J. Clin. Neurophysiol., 24(2):96-100 (2007a).

Tao, J., M. Baldwin, A. Ray, S. Hawes-Ebersole, y J. Ebersole. The impact of cerebral source area and synchrony on recording scalp electroencephalography ictal patterns. Epilepsia, 48(11):2167$2176(2007 \mathrm{~b})$.

Tao, J., A. Ray, S. Hawes-Ebersole, y J. Ebersole. Intracranial EEG substrates of scalp EEG interictal spikes. Epilepsia, 46(5):669-676 (2005).

Taoka, T., M. Morikawa, T. Akashi, T. Miyasaka, H. Nakagawa, K. Kiuchi, T. Kishimoto, y K. Kichikawa. Fractional anisotropy threshold dependence in tract-based diffusion tensor analysis: evaluation of the uncinate fasciculus in Alzheimer disease. AJNR Am. J. Neuroradiol., 30(9):1700-1703 (2009).

Tarkiainen, A., M. Liljestrom, M. Seppa, y R. Salmelin. The 3D topography of MEG source localization accuracy: effects of conductor model and noise. Clin. Neurophysiol., 114:1977-1992 (2003).

Telenczuk, B., S. Baker, A. Herz, y G. Curio. High-frequency EEG covaries with spike burst patterns detected in cortical neurons. J. Neurophysiol, 105(6):2951-2959 (2011).

ten Caat, M., N. Maurits, y J. Roerdink. Data-driven visualization and group analysis of multichannel EEG coherence with functional units. IEEE Trans. Viz. Comput. Graph., 20(4):756-771 (2008).

Tikhonov, A. y V. Arsenin. Solutions of Ill-posed Problems. Winston and Sons (1977).

Titomir, L. I. y P. Kneppo. Bioelectric and Biomagnetid Fields. CRC Press (1994).

Towle, V., J. Bolaños, D. Suarez, K. Tan, R. Grzeszczuk, D. Levin, R. Cakmur, S. Frank, y J. Spire. The spatial location of EEG electrodes: locating the best-fitting sphere relative to cortical anatomy. Electroenceph. Clin. Neurophysiol., 86:1-6 (1993).

Trisán-Vega, A., C.-F. Westin, y S. Aja-Fernández. Bias of least squares approaches for diffusion tensor estimation from array coils in DT-MRI. En Proc. 12th Intl. Conf. Med. Image Comp. 
Computer Assisted Interv. (MICCAI’09), págs. 919-926. London, UK (2009).

Tuch, D., V. Wedeen, A. Dale, J. George, y J. Belliveau. Conductivity tensor mapping of the human brain using diffusion tensor MRI. Proc. Natl. Acad. Sci. USA, 98(20):11697-11701 (2001).

Ulbert, I., E. Halgren, G. Heit, y G. Karmos. Multiple microelectrode-recording system for human intracortical applications. J. Neurosci. Methods, 106(1):69-79 (2001).

Utama, N., A. Takemoto, Y. Koike, y K. Nakamura. Phased processing of facial emotion: An ERP study. Neurosci. Res., 64:30-40 (2009).

Vaadia, E., I. Haalman, M. Abeles, H. Bergman, Y. Prut, H. Slovin, y A. Aertsen. Dynamics of neuronal interactions in monkey cortex in relation to behavioural events. Nature, 373:515-518 (1995).

Valdés-Hernández, P., N. von Ellenrieder, A. Ojeda-Gonzalez, S. Kochen, Y. Alemán-Gómez, C. Muravchik, y P. Valdés-Sosa. Approximate average head models for EEG source imaging. $J$. Neurosci. Methods, 185(1):125-132 (2009).

van den Broek, S., F. Reinders, M. Donderwinkel, y M. Peeters. Volume conduction effects in EEG and MEG. Electroenceph. Clin. Neurophysiol., 106:522-534 (1998).

van den Broek, S. P., H. Zhou, y M. J. Peters. Computation of neuromagnetic fields using finiteelements method and Biot-Savart law. Med. E Biol. Eng. E Comput., 34:21-26 (1996).

van der Loo, E., M. Congedo, M. Plazier, P. V. de Heyninga, y D. D. Ridder. Correlation between independent components of scalp EEG and intra-cranial EEG (iEEG) time series. Int. J. Bioelectromagnetism, 9(4):270-275 (2007).

van Trees, H. L. Detection, Estimation, and Modulation Theory. Part I. John Wiley and Sons (2001).

van Uitert, R., C. Johnson, y L. Zhukov. Influence of head tissue conductivity in forward and inverse magnetoencephalographic simulations using realistic head models. IEEE Trans. Biomed. Eng., 51(12):2129-2137 (2004).

van Veen, B., W. van Drongelen, M. Yuchtman, y A. Suzuki. Localization of brain electrical activity via linearly constrained minimum variance spatial filtering. IEEE Trans. Biomed. Eng., 44(9):867-880 (1997).

Vanrumste, B., G. V. Hoey, R. V. de Walle, M. D’Havé, I. Lemahieu, y P. Boon. The performance of the spherical head model compared to the realistic head model in dipole localization with noisy EEG. Med. Eng. Phys., 24:403-418 (2002).

Vanrumste, B., G. van Hoey, R. van de Walle, M. D'Havé, I. Lemahieu, y P. Boon. Dipole location errors in electroencephalogram source analysis due to volume conductor model errors. Med. 
Biol. Eng. Comput., 38:528-534 (2000).

Vanrumste, B., G. Van Hoey, R. Van de Walle, M. R. P. D'Havé, I. A. Lemahieu, y P. A. J. M. Boon. The validation of the finite difference method and reciprocity for solving the inverse problem in EEG dipole source analysis. Brain Topogr., 14(2):83-92 (2001).

Vatta, F., F. Meneghini, F. Esposito, S. Mininel, y F. D. S. F. Realistic and spherical head modeling for EEG forward problem solution: a comparative cortex-based analysis. Comput. Intell. Neurosci. (2010). Epub. 972060.

von Ellenrieder, N. Configuración Geométrica Aleatoria en Electromagnetoencefalografía. Tesis Doctoral, Facultad de Ingeniería, Universidad Nacional de La Plata, Argentina (2005).

von Ellenrieder, N., L. Beltrachini, A. Blenkmann, J. S. Martín, S. Kochen, y C. Muravchik. Determinación de la ubicación de electrodos de EEG basada en fotografías digitales. XIII Reunión de Trabajo en Procesamiento de la Información y Control (RPIC 2009), Rosario, Argentina, págs. 867-880 (2009a).

von Ellenrieder, N., L. Beltrachini, y C. Muravchik. Electrode and brain modeling in stereo-EEG. Clin. Neurophysiol. (2012). En Prensa.

von Ellenrieder, N. y C. Muravchik. Comparación entre sensores de potencial y gradiente de potencial en mediciones de EEG. En Actas del XVII Congreso Argentino de Control Automático (AADECA 2000), págs. 389-394. Buenos Aires, Argentina (2000).

von Ellenrieder, N., C. Muravchik, y A. Nehorai. MEG forward problem formulation using equivalent surface curent densities. IEEE Trans. Biomed. Eng., 52(7):1210-1217 (2005a).

von Ellenrieder, N., C. Muravchik, y A. Nehorai. Effects of geometric head model perturbations on the EEG inverse problem. IEEE Trans. Biomed. Eng., 53(2):249-257 (2006).

von Ellenrieder, N., C. Muravchik, M. Wagner, y A. Nehorai. Effect of head shape variations among individuals on the EEG/MEG forward and inverse problems. IEEE Trans. Biomed. Eng., 56(3):587-597 (2009b).

von Ellenrieder, N., C. H. Muravchik, y A. Nehorai. A meshless method for solving the EEG forward problem. IEEE Trans. Biomed. Eng., 52(2):249-257 (2005b).

von Ellenrieder, N., P. Valdés-Hernández, y C. Muravchik. On the EEG/MEG forward problem solution for distributed cortical sources. Med. Biol. Eng. Comp., 47(10):1083-1091 (2009c).

Vrba, J. y S. Robinson. Differences between synthetic aperture magnetometry (SAM) and linear beamformers. En Proc. 12th Intl. Conf. Biomag., págs. 681-684. Helsinki University of Technology, Espoo, Finland (2000).

Vuilleumier, P. y G. Pourtois. Distributed and interactive brain mechanisms during emotion face 
perception: Evidence from functional neuroimaging. Neuropsychologia, 45:174-194 (2007).

Waldorp, L., H. Huizenga, C. Dolan, y P. Molenaar. Estimated generalized least squares electromagnetic source analysis based on a parametric noise covariance model. IEEE Trans. Biomed. Eng., 48:737-741 (2002).

Wang, J., T. Chao, Y. Wai, y Y. Hsu. Novel diffusion anisotropy inices: an evaluation. J. Magn. Res., 24:211-217 (2006).

Wang, M. y J. Gotman. The influence of electrode location errors on EEG dipole source localization with a realistic head model. Clin. Neurophysiol., 112:1777-1780 (2001).

Wang, M., C. Maurer, J. Fitzpatrick, y R. Maciunas. An automatic technique for finding and localizing externally attached markers in CT and MR volume images of the head. IEEE Trans. Biomed. Eng., 43(6):627-637 (1996).

Watanabe, S., R. Kakigi, y A. Puce. The spatiotemporal dynamics of the face inversion effect: a magneto- and electro-encephalographic study. Neuroscience, 116:879-895 (2003).

Weber, D., C. Clark, A. McFarlane, K. Moores, P. Morris, y G. Egan. Abnormal frontal and parietal activity during working memory updating in post-traumatic stress disorder. Psych. Res. Neuroimag., 140:27-44 (2005).

Wendel, K. y J. Malmivuo. Correlation between live and post mortem skull conductivity measurements. En Conf. Proc. IEEE Eng. Med. Biol. Soc., págs. 4285-4288. New York, USA (2006).

Wendel, K., N. Narra, M. Hannula, P. Kauppinen, y J. Malmivuo. The influence of CSF on EEG sensitivity distributions of multilayered head models. IEEE Trans. Biomed. Eng., 55:1454-1456 (2008).

Westin, C.-F., S. Peled, H. Gudbjartsson, R. Kikinis, y F. A. Jolesz. Geometrical diffusion measures for MRI from tensor basis analysis. En ISMRM '97, pág. 1742. Vancouver Canada (1997).

Wolters, C., A. Anwwander, X. Tricoche, D. Weinstein, M. Koch, y R. MacLeod. Influence of tissue conductivity anisotropy on EEG/MEG field and return current computation in a realistic head model: A simulation and visualization study using high-resolution finite element modeling. Neuroimage, 30(3):813-826 (2006).

Wolters, C., H. Köstler, C. Möller, J. Härdtlein, L. Grasedyck, y W. Hackbusch. Numerical mathematics of the substraction method for the modeling of a current dipole in EEG source reconstruction using finite element head models. SIAM J. Sci. Comput., 30(1):24-45 (2007).

Xu, D., J. Cui, R. Bansal, X. Hao, J. Liu, W. Chen, y B. Peterson. The ellipsoidal area ratio: an alternative anisotropy index for DTI. Magn. Reson. Imag., 27:311-323 (2009).

Yamazaki, M., D. Tucker, A. Fujimoto, T. Yamazoe, T. Okanishi, T. Yokota, H. Enoki, y T. Ya- 
mamoto. Comparison of dense array EEG with simultaneous intracranial EEG for interictal spike detection and localization. Epilepsy Research (2011). In press.

Yan, Y., P. L. Nunez, y R. T. Hart. Finite-element model of the human head: scalp potentials due to dipole sources. Med. \& Biol. Eng. \& Comput., 29:475-481 (1991).

Yang, J., X. Ma, C. Hou, y Y. Liu. Automatic generalized loading for robust adaptive beamforming. IEEE Signal Porcess. Letters, 16(3):219-222 (2009).

Yetik, I., A. Nehorai, C. Muravchik, y J. Haueisen. Line source modeling and estimation with magnetoencephalography. IEEE Trans Biomed Eng., 52(5):839-851 (2005).

Yetik, I., A. Nehorai, C. Muravchik, J. Haueisen, y M. Eiselt. Surface-source modeling and estimation using biomagnetic measurements. IEEE Trans. Biomed. Eng., 53(10):1872-1882 (2006).

Yoo, S., C. R. Guttmann, J. Ives, L. Panych, R. Kikinis, D. Schomer, y F. Jolesz. 3D localization of surface 10-20 EEG electrodes on high resolution anatomical MR images. Electroenceph. Clin. Neurophisiol., 102:335-339 (1997).

Yvert, B., O. Bertrand, M. Thévenet, J. F. Echallier, y J. Pernier. A systematic evaluation of the spherical model accuracy in EEG dipole localization. Electroenceph. Clin. Neurophysiol., 102:452-459 (1997).

Zhang, S., H. Peng, R. Dawe, y K. Arfanakis. Enhanced ICBM diffusion tensor template of the human brain. Neuroimage, 54:974-984 (2011).

Zhang, Y., L. Ding, W. van Drongelen, K. Hecox, D. Frim, y B. He. A cortical potential imaging study from simultaneous extra- and intracranial electrical recordings by means of the finite element method. Neuroimage, 31:1513-1524 (2006a).

Zhang, Y., D. Sun, y D. Zhang. Robust adaptive acoustic vector sensor beamforming using automated diagonal loading. Applied Acoustics, 70:1029-1033 (2009).

Zhang, Y., W. van Drongelen, y B. He. Estimation of in vivo brain-to-skull conductivity ratio in humans. Appl. Phys. Lett., 89(4):223903-2239033 (2006b).

Zhang, Y., S. Zhu, y B. He. A second-order finite element algorithm for solving the threedimensional EEG forward problem. Phys. Med. Biol., 49:2975-2987 (2004).

Zhang, Z. A fast method to compute surface potentials generated by dipoles within multilayer anisotropic spheres. Phys. Med. and Biol., 40:335-349 (1995).

Zhou, H. y A. van Oosterom. Computation of the potential distribution in a four-layer anisotropic concentric spherical volume conductor. IEEE Trans. Biomed. Eng., 39:154-158 (1992).

Zhu, X., J. Li, y P. Stoica. Knowledge-aided adaptive beamforming. Signal Processing, IET, $2(4): 335-345(2008)$. 